\title{
Singularities of two-point functions in Quantum Field Theory
}

\author{
Dissertation \\ zur Erlangung des mathematisch-naturwissenschaftlichen Doktorgrades \\ "Doctor rerum naturalium" (Dr. rer. nat.) \\ der Georg-August-Universität Göttingen \\ im Promotionsprogramm GTK 1493 \\ der Georg-August University School of Science (GAUSS)
}

vorgelegt von

Michał Wrochna

aus Warszawa

Göttingen 2013 


\section{Betreuungsausschuss}

Prof. Dr. Dorothea Bahns (Mathematisches Institut)

Prof. Dr. Ingo Witt (Mathematisches Institut)

\section{Prüfungskommission}

Referent: Prof. Dr. Dorothea Bahns

Korreferent: Prof. Dr. Ingo Witt

Weitere mitglieder der Prüfungskommission:

Prof. Dr. Karl-Henning Rehren (Theoretische Physik)

Prof. Dr. Thomas Schick (Mathematisches Institut)

Prof. Dr. Russell Luke (Numerische und Angewandte Mathematik)

Prof. Dr. Anja Sturm (Mathematische Stochastik)

Tag der mündlichen Prüfung: 16.08.13 


\begin{abstract}
The main topic of the present thesis is the study of singularities of two-point functions of spin-0 and spin- $\frac{1}{2}$ quantum fields, possibly set on curved spacetime or in the presence of smooth, external electromagnetic potentials.

The first part reviews the results necessary for the construction of neutral and charged non-interacting quantum fields on globally hyperbolic spacetimes, and supplements the arguments needed in the case when no charge symmetry exists. In this general situation, the Hadamard condition, which refers to the singularities of the two-point functions, is discussed, and its relation to the theory of distinguished parametrices of Duistermaat and Hörmander is explained. Additionally, similarities between the spin- 0 and spin- $\frac{1}{2}$ case are exhibited by considering a two-component form of the Klein-Gordon equation. It is then used in the static case to reformulate the classical dynamics as an evolution equation whose generator is self-adjoint in the sense of Krein spaces. By methods of spectral theory in Krein space, we construct Hadamard two-point functions in the spin-0 case for a class of strong electric potentials which possess no ground state.

The second part is concerned with renormalisation of interacting fields in the approach of Epstein and Glaser. We focus on the problem of recovering symmetries, possibly lost in the process of extending singular distributions on $\mathbb{R}^{n} \backslash\{0\}$ to $\mathbb{R}^{n}$. In our approach, this is done by imposing that the extended distributions are in the kernel of a given set of (differential) operators. The symmetries are then recovered using a map, which in typical applications turns out to be linear. The same method is applied to derive the relation between off-shell and on-shell time-ordered products for a scalar theory on Minkowski space.
\end{abstract}





\section{Acknowledgments}

I would like to thank my supervisor Dorothea Bahns, who guided me through the writing process with great compassion and optimism. I am grateful to Ingo Witt for all the stimulating discussions and the numerous seminars that helped me get a grasp of microlocal analysis. I owe my deepest gratitude to Jan Dereziński, who introduced me

to the broad world of mathematical physics, and discussions with whom have always been a valuable source of inspiration. I am also indebted to all who contributed to the present work by providing critical comments, constructive remarks and encouraging feedback, in that respect I would like to single out for special thanks Michael Dütsch, Christian Gérard, Nicola Pinamonti and Jochen Zahn.

It is a pleasure to thank all my colleagues, without whom Göttingen would not be the same place, especially Daniela, Gennaro, Huong, Luca, Nils, Olivier, René, Sutanu, Thang and Zheng. Financial support of the Research Training Group 1493 "Mathematical Structures in Modern Quantum Physics" is gratefully acknowledged.

Last but not least, I cannot thank my family and close friends enough for their

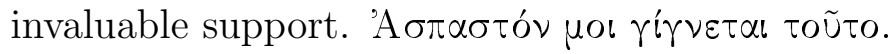




\section{Contents}

Introduction 1

Part 1. Microlocal description of singularities: Two-point functions in non-interacting QFT

Chapter 1. Non-interacting quantum fields 9

1.1. Bilinear and sesquilinear forms 10

1.2. Symplectic and unitary group $\quad 12$

1.3. Representations of CARs and CCRs 13

1.4. CAR and CCR $C^{*}$-algebras $\quad 16$

$\begin{array}{ll}\text { 1.5. Quasi-free states } & 18\end{array}$

Chapter 2. Normally and pre-normally hyperbolic operators 29

2.1. Globally hyperbolic spacetimes 30

2.2. Distributions on manifolds 32

2.3. Operators on manifolds 32

2.4. Normally and pre-normally hyperbolic operators 34

2.5. The Dirac equation in external potentials 43

2.6. Two-component form of the Klein-Gordon equation 46

2.7. Distributional two-point functions 49

Chapter 3. The Hadamard condition $\quad 51$

3.1. Parametrices $\quad 52$

3.2. Wave front set $\quad 52$

3.3. Distinguished parametrices 55

3.4. The Hadamard condition $\quad 59$

3.5. Existence and examples of Hadamard states 62

Chapter 4. Dirac equation in static external potentials 65

4.1. Static spacetimes 65

4.2. Abstract Dirac equations 66

$\begin{array}{ll}\text { 4.3. Hadamard states } & 67\end{array}$

Chapter 5. Klein-Gordon equation in static external potentials 71

5.1. Abstract Klein-Gordon equation $\quad 71$

$\begin{array}{ll}\text { 5.2. Definitizable operators } & 77\end{array}$

5.3. Hadamard states 81

Part 2. Strength of singularities: Renormalisation and interacting fields $\quad 85$

$\begin{array}{lll}\text { Chapter 6. } & \text { Renormalisation and scaling degree } & 87\end{array}$ 
6.1. The algebra of Wick polynomials $\quad 87$

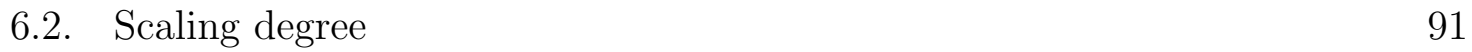

Chapter 7. On-shell extension of distributions 95

7.1. Operators of finite essential order 97

7.2. On-shell extension — single operator case 99

7.3. On-shell extension — multiple operators 106

Chapter 8. On-shell and off-shell time-ordered products 111

8.1. Map relating off-shell and on-shell time-ordered products 112

$\begin{array}{ll}\text { Bibliography } & 117\end{array}$

-vii- 
"I am not interested in erecting a building, but in perspicuously presenting to myself the foundations of all possible buildings."

— Ludwig Wittgenstein 


\section{Introduction}

The implementation of the principle of locality in Quantum Field Theory has led to profound theoretical insights, resulting on one side in axiomatic descriptions of the non-interacting theory, emphasizing the structure of the underlying space-time, and on the other side in a rigorous construction of interacting fields in position space, known as the Epstein and Glaser method. At present, these two fundamental ingredients are unified in a local, perturbative formulation of QFT, that can be set both on curved spacetime and in external electromagnetic potentials, without the need to rely on any special symmetries of the system, nor on concepts such as implementability of the classical evolution in Fock space.

In a mathematical physicist's terminology, the non-interacting theory is described by a representation of the canonical commutation or anti-commutation relations (abbreviated CCR or CAR). This is understood as a map $V \ni v \mapsto \psi(v)$ from a vector space $V$ to operators on a Hilbert space $\mathcal{H}$, such that $\psi(v)$ satisfy (anti-) commutation relations consistent with an additional structure on $V$ derived from the classical theory, for instance a symplectic form. In the quantum field theoretical context, $V$ can be identified with a subspace of smooth functions on the space-time $M$ and in typical situations it is possible to interpret $v \mapsto \psi(v)$ as an operator-valued distribution, symbolically denoted $\psi(x)$. The perturbative formulation of interacting

QFT raises the question of how to define powers and pointwise products of $\psi(x)$ and $\psi^{*}(x)$, such as for instance $\psi(x) \psi^{*}(x)$. The distributions involved being too singular, this problem cannot be solved without a renormalization prescription - an example is provided by the commonly used normal ordering on Minkowski space, which is properly generalized by the point-splitting procedure. This rather universal method consists of selecting a distribution $H(x, y)$ such that the limit

$$
: \psi \psi^{*}(x):_{H}:=\lim _{x \rightarrow y}\left(\psi(x) \psi^{*}(y)-H(x, y) \mathbf{1}\right)
$$

exists at least weakly, on a dense subset of $\mathcal{H}$ which is required to include a given distinguished vector $\Psi$, interpreted as the vacuum of the theory. This is possible whenever the singularities of the distributions $H(x, y)$ and $\left(\Psi \mid \psi(x) \psi^{*}(y) \Psi\right)$ cancel out, therefore special significance is attributed to the singular structure of the twopoint function $\left(\Psi \mid \psi(x) \psi^{*}(y) \Psi\right)$.

Since the properties of $\Psi$ and $\psi(x)$ strongly rely on the underlying Hilbert space structure $\mathcal{H}$, it is useful to consider a more representation-independent formalism, as provided by the algebraic approach of Haag and Kastler [Haa59, HK64]. In this setting, field operators $\psi(v)$ are replaced by abstract elements of a $C^{*}$-algebra that 
encode the CARs or CCRs, and the usual Hilbert space picture is recovered after choosing a state $\omega$ and applying the GNS construction. Of particular interest are quasi-free states, which are uniquely determined by the sesquilinear form $v, w \mapsto$ $\omega\left(\psi(v) \psi^{*}(w)\right)$, also called the two-point function in what follows.

On Minkowski space, the choice of a state is uniquely fixed upon requiring its translation invariance - this yields the Minkowski vacuum $\omega_{\mathrm{vac}}$. The standard choice in renormalisation consists of taking $H(x, y)=\omega_{\mathrm{vac}}\left(\psi(x) \psi^{*}(y)\right)$, then the evaluation of $\omega_{\text {vac }}$ on the Wick product $: \psi \psi^{*}:_{H}$ (defined by point-splitting) is equal to zero, whereas expectation values for higher Wick products can be computed by means of the Wick theorem. The interacting theory can be then studied using various methods, most of which are based on explicit formulae for the Fourier transform of $\omega_{\text {vac }}\left(\psi(x) \psi^{*}(y)\right)$ in the difference variables $x-y$. In axiomatic approaches, the essential property of the Minkowski vacuum is the spectral condition, that states that the joint spectrum of generators of translation in the GNS representation of $\omega_{\text {vac }}$ is contained in the forward lightcone.

On a generic curved background or in the presence of external potentials, neither special symmetries nor Fourier analysis can be directly employed, and the existence of a property that could appropriately generalise the spectral condition remained an open problem for years. Special insight was provided by works on renormalisation of the quantum stress-energy tensor [Wal77]. It was realized that the so-called Hadamard parametrix not only encodes the singularities of the two-point function of $\omega_{\mathrm{vac}}$ on Minkowski space, but it can also be used to define on curved spacetime a class of states whose two-point function have essentially the same short-distance behaviour - the so-called Hadamard states. This observation stems from the fact that by construction, two-point functions solve the Klein-Gordon or Dirac equation in both variables $x$ and $y$, and that Hadamard's method for obtaining approximate solutions of these equations can be tailored to yield a generalization of $\omega_{\text {vac }}\left(\psi(x) \psi^{*}(y)\right)$.

Although the Hadamard parametrix can be written down explicitly, it provides a description of singularities that did not seem very appealing from the mathematical point of view and which proved to be difficult to apply in practice, making the study of generic properties of Hadamard states a challenging task. The situation changed dramatically with the work of Radzikowski, who rephrased the Hadamard condition in the language of microlocal analysis [Rad96]. He proved that two-point functions of Hadamard states can be equivalently characterized by a condition on their wave front set, a refinement of the singular support of a distribution that additionally indicates the directions in momentum space responsible for singularities. This insight initiated a series of important results, including the construction of Wick monomials on curved spacetime [BFK96], the proof of a generalized spin-statistics theorem [Ver01], and most importantly, the construction of renormalised interacting QFT based on Hadamard states by Brunetti and Fredenhagen [BF00].

The first part of the thesis is concerned with singularities of distributional twopoint functions from the microlocal point of view. The literature on the microlocal 
formulation of the Hadamard condition and Radzikowski's theorem is extensive and includes up-to-date reviews such as [San08, Hac10], it does however only treat, with only very few exceptions [Hol01, Mar03, Zah13], the special case of states that are invariant under charge conjugation. One of our main aims is to supplement the arguments necessary to treat the general case, and to exhibit at the same time as many common features of spin- 0 and spin- $\frac{1}{2}$ fields on curved spacetime. Since existence of a charge conjugation is not assumed, this allows us to extend known results to the case of charged fields, minimally coupled to an external, smooth electromagnetic potential.

We start by recalling in Chapter 1 conditions for a generic sesquilinear form to be the two-point function of a (pure, gauge-invariant) quasi-free state, following textbook references such as [DG13]. A particular emphasis is put on the relation between neutral and charge fields and it is shown that the study of quasi-free states can always be reduced to charged ones, in which case the two-point function is a hermitian form on a complex vector space. This is particularly useful in the construction of Hadamard states, since the methods of microlocal analysis are not very well adapted to real function spaces.

Chapter 2 contains the ingredients needed for the construction of non-interacting spin-0 and spin-1/2 fields on globally hyperbolic spacetimes and in the presence of smooth external potentials. We argue that similarities between the spin- 0 and spin$1 / 2$ case are best exhibited by replacing the Klein-Gordon equation with an equivalent equation, that on $d$-dimensional Minkowski space reads

$$
\left(\mathrm{i} \partial_{t}+B\right)\left(\begin{array}{l}
f_{0} \\
f_{1}
\end{array}\right)=0, \quad B=\left(\begin{array}{cc}
V & \mathbf{1} \\
-\sum_{i=1}^{d}\left(\partial_{i}+\mathrm{i} A_{i}\right)^{2}+m^{2} & V
\end{array}\right),
$$

and which we call the two-component Klein-Gordon equation. Although the generalization to curved backgrounds requires us to assume additionally the existence of a Gaussian foliation of the underlying spacetime, the two-component form turns out to be advantageous in deriving formulae for the solution of the Cauchy problem associated to the usual one-component form.

The Hadamard condition and its connection to the theory of distinguished parametrices of Duistermaat and Hörmander are reviewed in Chapter 3. As observed by Hollands [Hol01], if invariance under charge conjugation is not assumed, the correct formulation of the Hadamard condition refers to both the wave front sets of $\omega\left(\psi(x) \psi^{*}(y)\right)$ and $\omega\left(\psi^{*}(x) \psi(y)\right)$. Our main contribution is a proof of Radzikowski's result adapted to this general case.

Next, in Chapters 4 and 5, we focus on the static case, allowing also for timeindependent electromagnetic potentials. Although many references discuss ground states and their Hadamard property is well-known [FNW81, SV00, SV01, SVW02, San12b], they do not cover the case of the Klein-Gordon equation in an overcritical electric potential, when ground state are known not to exist. Under assumptions on the $L^{\infty}$ norm and decay at infinity of the electric potential, it is possible to interpret the two-component Klein-Gordon equation as an evolution equation whose generator is a Krein self-adjoint operator $B$ in a Krein space [LNT08]. Although in the 
overcritical case, $B$ acquires non-real eigenvalues, the corresponding eigenspace can be decoupled from the system if $B$ belongs to the class of definitizable operators. We make use of results from Krein-space spectral theory and define quasi-free states, that have properties analogous to ground states on the subsystem with no complex eigenvalues, and we prove that they satisfy the Hadamard condition. This generalises our prior results obtained in [Wro12] in the flat case, and is also an improvement in the respect that the present construction yields gauge-invariant states.

This way, our results confirm the common expectation that methods originally developed for curved spacetime can be efficiently adapted to QFT in smooth external potentials, even in singular cases such as spin-0 fields in overcritical electric potentials.

In the second part of the thesis, we investigate mathematical issues connected to renormalisation in interacting theory in the setting of Epstein and Glaser [EG73]. In local perturbation theory, once Wick powers $: \psi^{k}:(x)$ (and their derivatives) are constructed, physical quantities are derived from the $S$-matrix, defined as a formal power series

$$
S(\eta)=\mathbf{1}+\sum_{k=1}^{\infty} \frac{\mathrm{i}^{k}}{k !} \int_{M \times k} T\left(\mathscr{L}\left(x_{1}\right), \ldots, \mathscr{L}\left(x_{k}\right)\right) \eta\left(x_{1}\right) \ldots \eta\left(x_{k}\right) d \Omega_{g}^{k},
$$

where the Lagrangian density $\mathscr{L}(x)$ is a Wick polynomial and $\eta$ is a test function which serves as an infrared cutoff. The time-ordered products $T$ are maps defined by a set of axioms for the $S$-matrix, attributed to Bogoliubov and Shirkov [BS59], such as symmetry, causality, etc. By means of the Wick expansion, it is possible to reduce the problem of determining the operator-valued distributions $T\left(\mathscr{L}\left(x_{1}\right), \ldots, \mathscr{L}\left(x_{k}\right)\right)$ to a similar problem for the ordinary distributions $\left(\Psi \mid T\left(\mathscr{L}\left(x_{1}\right), \ldots, \mathscr{L}\left(x_{k}\right)\right) \Psi\right)$. In this reduced setting, it is either possible to work with on-shell time-ordered products $T_{\mathrm{on}}$, that are consistent with the free equations of motion, or with off-shell ones $T_{\text {off }}$, that commute with derivatives and for this reason are often more useful in practice. In both cases, the distributions to be determined are constructed in an iterative procedure, that can however be made precise only if one disregards points on the diagonal in $M^{\times k}$. For instance, on Minkowski space it yields products of distributions such as the square of $\theta\left(x_{0}-y_{0}\right)\left(\Psi \mid \psi(x) \psi^{*}(y) \Psi\right)$, where $\theta$ is the Heaviside step function. Although the Hadamard condition can be used in this case to define the products outside of $x_{0} \neq y_{0}$, the result still needs to be extended to $x_{0}-y_{0}$ and the latter operation is ambiguous - this accounts for the renormalisation freedom well-known from other approaches. Making either use of translation-invariance in the Minkowski case, or of an expansion due to Hollands and Wald in the general case [HW02], the construction of time-ordered products amounts to extending distributions defined originally on $\mathbb{R}^{n} \backslash\{0\}$ to the whole space $\mathbb{R}^{n}$. The ambiguities can be constrained by requiring that the strength of the singularity at the origin is not significantly affected in the extension process. As explained in Chapter 6, this is most efficiently done using Steinmann's notion of scaling degree of a distribution [Ste71]. 
The final step in the construction of the $S$-matrix consists of proving existence of extensions subject to further renormalisation conditions, such as Lorentz invariance, invariance under discrete symmetries, etc., a task that has been achieved in the physically relevant examples on a case-by-case basis [Sch95, Pra99, HW02, Gra03, LG03, DF04]. In Chapter 6, we present an alternative, and rather universal method developed by us recently in collaboration with Bahns [BW12], inspired by the treatment of Lorentz invariance in the work of Dütsch and Fredenhagen [DF04]. The main idea is to reformulate renormalisation conditions as the requirement that the extended distributions lie in the kernel of a given set of operators $\left\{R^{i}\right\}_{i \in \mathcal{I}}$. In QFT these are typically differential operators, for instance infinitesimal generators of Lie groups, that act on $\mathbb{R}^{n}$ in such way that $\{0\}$ is a fixed point. It turns out that the problem can be reduced to a finite-dimensional one by considering the restriction of $R^{i}$ to the space spanned by derivatives of the Dirac delta distribution $\delta$ up to a certain order, and conventional linear algebra can be applied.

The same methods are used in Chapter 8 to solve an apparently unrelated problem, namely the precise relation between on-shell and off-shell time-ordered products, $T_{\text {on }}$ and $T_{\text {off }}$, in the case of a scalar theory on Minkowski space. Although an explicit solution was already given by Brouder and Dütsch [BD08], our method avoids long combinatorial expression and relies instead on spectral properties of finite-dimensional operators, directly related to the Klein-Gordon equation.

The main consequence of our results is that in local perturbative QFT, the global symmetries (and other renormalisation conditions, including the on-shell property), possibly lost in the extension process, can be recovered by applying one local, linear map. This exhibits yet another feature of the Epstein and Glaser method, which we attribute once again to the principle of locality. 



\section{Part 1}

\section{Microlocal description of singularities: \\ Two-point functions in non-interacting QFT}





\section{CHAPTER 1}

\section{Non-interacting quantum fields}

Both in the context of Quantum Mechanics and non-interacting Quantum Field Theory, quantization can be viewed as the construction of field operators $\psi(v)$ on a Hilbert space, parametrized by elements $v$ of a real or complex vector space $V$. Field operators are required to satisfy a set of commutation or anti-commutation relations induced from an additional structure on $V$, namely a symplectic form in the case of neutral bosons or a hermitian positive form for charged fermions, which has the interpretation of the (dual) phase space of the classical theory.

For the sake of clarity let us illustrate this on the example of charged fermionic fields.

Let $(V, q(\cdot, \cdot))$ be a complex vector space equipped with a positive hermitian sesquilinear form (in other words, a pre-Hilbert space) and let $\mathcal{H}$ be a Hilbert space. A charged representation of the $C A R \mathrm{~s}$ in $\mathcal{H}$ is an anti-linear map

$$
v \ni V \mapsto \psi(v) \in \mathcal{B}(\mathcal{H})
$$

which satisfies the canonical anti-commutation relations:

$$
\begin{array}{ll} 
& \left\{\psi^{*}(v), \psi^{*}(w)\right\}=\{\psi(v), \psi(w)\}=0, \\
(C A R) \quad & \left\{\psi(v), \psi^{*}(w)\right\}=q(v, w) \mathbf{1}, \quad v, w \in V,
\end{array}
$$

where the curly bracket stands for the anti-commutator of bounded operators. In Quantum Field Theory, $V$ is always infinite dimensional and there exist many unitarily non-equivalent representations of the CARs. An efficient framework aimed to deal with this problem is provided by the algebraic approach, initiated by Haag and Kastler [Haa59, HK64]. The first step consists of defining a $C^{*}$-algebras $\operatorname{CAR}(V, q)$ spanned by abstract elements $\phi(v)$ subject to the relations $(C A R)$. After choosing a state $\omega$ on this $C^{*}$-algebra, one obtains a CAR representation by applying the GNS construction.

The simplest class of states are quasi-free ones. They have the property that they are uniquely determined by their action on elements of the form $\psi^{*}(v) \psi(w)$. The sesquilinear form $\lambda(v, w)=\omega\left(\psi^{*}(v) \psi(w)\right)$, called the two-point function of $\omega$, is thus of particular interest, and it is possible to characterize sesquilinear forms which are two-point functions of states with specific properties.

In this chapter, we introduce the basic material on representations of canonical (anti-) commutation relations, quasi-free states and their two-point functions. Our presentation of the subject follows closely [Der06, DG13] (other textbook references we use are [BR97, BSZ92]) and includes a systematic treatment of the four distinct cases: neutral/charged fermions and neutral/charged bosons. A particular emphasis 
is put on the relation between the neutral and the charged case. As discussed in detail in [GW13] in the bosonic case, it is possible to set up an equivalence between two-point functions in the neutral case and two-point functions of gauge-invariant quasi-free state in the charged case. This observation will allow us to restrict to the charged case uniquely in the next chapters, which is particularly convenient as one avoids this way the use of real vector spaces and real forms, not much compatible with the techniques of microlocal analysis.

The notations and conventions we adopt are rather standard ones, except that in the charge bosonic case, instead of commonly used anti-hermitian sesquilinear forms ('complex symplectic spaces') we rather consider hermitian ones. This is only a matter of convenience and serves us to have notations which are more coherent with the fermionic case.

\subsection{Bilinear and sesquilinear forms}

If $V, W$ are vector spaces over $\mathbb{K}=\mathbb{R}$ or $\mathbb{C}$, we denote $L(V, W)$ the space of all $\mathbb{K}$-linear maps from $V$ to $W$, and set $L(V)=L(V, V)$.

Let $X$ be a vector space over $\mathbb{R}$. Its dual $X^{\#}$ is the vector space of all linear functionals on $X$. In what follows, the evaluation of an anti-linear functional $z \in X^{\#}$ on an element $f \in X$ will be denoted $\langle z, f\rangle \in \mathbb{R}$.

Bilinear forms on $X$ can be defined as elements of $L\left(X, X^{\#}\right)$. To each such $\beta \in L\left(X, X^{\#}\right)$ we associate a map

$$
\begin{aligned}
& \beta(\cdot, \cdot): X \times X \rightarrow \mathbb{R} \\
& \beta(f, g):=\langle\beta f, g\rangle,
\end{aligned}
$$

which is bilinear in the usual sense. We will use interchangeably the notation $\beta$ or $\beta(\cdot, \cdot)$ to denote elements of $L\left(X, X^{\#}\right)$ - the 'operator-like' notation is more convenient for writing operations such as composition with a linear maps, whereas the 'bilinear' notation is often more practical when evaluation on specific vectors $f, g \in X$ is needed to be displayed without referring to the dual space $X^{\#}$.

Definition 1.1.1. Let $\beta \in L\left(X, X^{\#}\right)$ be a bilinear form. One says that:

- $\beta$ is symmetric if $\beta(f, g)=\beta(g, f)$ for all $f, g \in X$;

- $\beta$ is anti-symmetric if $\beta(f, g)=-\beta(f, g)$ for all $f, g \in X$;

- $\beta$ is non-degenerate if $\operatorname{Ker} \beta=\{0\}$, or equivalently if for any $f \in X$

$$
\beta(f, g)=0 \quad \forall g \in X
$$

implies $f=0$;

- $\beta$ is positive if it is symmetric and $\beta(f, f)>0$ for all $f \neq 0$. In such case the pair $(X, \beta)$ is called an orthogonal space.

- $\beta$ is a symplectic form if it is anti-symmetric and non-degenerate. In such case the pair $(X, \beta)$ is called a symplectic space. 
Let $V$ be a vector space over $\mathbb{C}$. In such case we denote $V^{*}$ its dual and the terminology just introduced for the real case extends directly. However, it is often more convenient to consider sesquilinear forms rather than bilinear ones and some more terminology needs to be introduced for that purpose.

The anti-dual $\bar{V}^{*}$ is the vector space of all anti-linear functionals on $V$. In what follows, the evaluation of an anti-linear functional $z \in \bar{V}^{*}$ on an element $v \in V$ will be denoted $\langle z, v\rangle \in \mathbb{C}$.

Sesquilinear forms on $V$ can be defined as elements of $L\left(V, \bar{V}^{*}\right)$. To each such $\alpha \in L\left(V, \bar{V}^{*}\right)$ we associate a map

$$
\begin{aligned}
& \alpha(\cdot, \cdot): V \times V \rightarrow \mathbb{C} \\
& \alpha(v, w):=\langle\alpha v, w\rangle,
\end{aligned}
$$

which is sesquilinear in the sense that it is anti-linear in the first argument and linear in the second argument. We will use intercheangably the notation $\alpha$ or $\alpha(\cdot, \cdot)$ to denote elements of $L\left(V, \bar{V}^{*}\right)$.

Definition 1.1.2. Let $\alpha \in L\left(V, \bar{V}^{*}\right)$ be a sesquilinear form. One says that

- $\alpha$ is hermitian if $\alpha(w, v)=\overline{\alpha(v, w)}$ for all $v, w \in V$;

- $\alpha$ is anti-hermitian if $\alpha(w, v)=-\overline{\alpha(v, w)}$ for all $v, w \in V$;

- $\alpha$ is non-degenerate if Ker $\alpha=\{0\}$, or equivalently if for any $v \in V$,

$$
\alpha(v, w)=0 \quad \forall w \in V
$$

implies $v=0$.

- $\alpha$ is positive if it is hermitian and $\alpha(v, v)>0$ for all $v \neq 0$. In that case we write $\alpha \geq 0$.

A positive sesquilinear form is also called a scalar product. Note that it is always a non-degenerate form.

Definition 1.1.3. A pre-unitary space is a pair $(V, \alpha)$ which consists of a complex vector space $V$ and a non-degenerate hermitian form $\alpha \in L\left(V, \bar{V}^{*}\right)$.

In the context of quantization one sometimes introduce 'complex symplectic' forms, which are anti-hermitian and non-degenerate. Observe however that if $\sigma$ is such an anti-hermitian form then $\alpha:=\mathrm{i} \sigma$ is hermitian, thus in practice it suffices to work with non-degenerate hermitian forms and pre-unitary spaces.

Let us now introduce the notions needed to pass from real forms to complex ones and vice-versa.

If $X$ is a real vector space, we denote $\mathbb{C} X=X \otimes_{\mathbb{R}} \mathbb{C}$ its complexification. If $\beta$ is a bilinear form on the real vector space $X$, its canonical sesquilinear extension to $\mathbb{C} X$ is the sesquilinear form $\beta_{\mathbb{C}}$ on $\mathbb{C} X$ given by

$$
\beta_{\mathbb{C}}\left(w_{1}, w_{2}\right):=\beta\left(f_{1}, f_{2}\right)+\beta\left(g_{1}, g_{2}\right)+\mathrm{i} \beta\left(f_{1}, g_{2}\right)-\mathrm{i} \beta\left(g_{1}, f_{2}\right), \quad w_{i}=f_{i}+\mathrm{i} g_{i}
$$

for $f_{i}, g_{i} \in X, i=1,2$. The assignment $\beta \mapsto \beta_{\mathbb{C}}$ maps (anti-)symmetric forms on $X$ onto (anti-)hermitian forms on the complexified space $\mathbb{C} X$. 
Conversely if $V$ is a complex vector space, then we denote $V_{\mathbb{R}}$ its real form, i.e., $V$ considered as a real vector space (we simply forget about the complex structure). Then given a sesquilinear form $\alpha \in L\left(V, \bar{V}^{*}\right)$ one obtains a bilinear form on $V_{\mathbb{R}}$ by taking the real part $\operatorname{Re} \alpha$. Clearly, the assignment $\alpha \mapsto \operatorname{Re} \alpha$ maps (anti-)hermitian forms on $V$ onto (anti-)symmetric forms on the real space $V_{\mathbb{R}}$.

It is easy to see that if $\beta \in L\left(X, X^{\#}\right)$ is positive then its canonical sesquilinear extension $\beta_{\mathbb{C}} \in L\left(\mathbb{C} X, \overline{\mathbb{C} X}^{*}\right)$ is positive. The converse statement requires a little more care because given a sesquilinear form $\alpha \in L\left(\mathbb{C} X, \overline{\mathbb{C} X}^{*}\right)$ it is a priori not clear whether it can be obtained as the sesquilinear extension of a bilinear form $\beta \in L\left(X, X^{\#}\right)$.

Lemma 1.1.4. Let $X$ be a real vector space and let $\alpha$ be a hermitian sesquilinear form on $\mathbb{C} X$. Then $\alpha=\beta_{\mathbb{C}}$ for some $\beta \in L\left(X, X^{\#}\right)$ with $\beta \geq 0$ iff $\alpha \geq 0$.

\subsection{Symplectic and unitary group}

Definition 1.2.1. Let $X, Y$ be real vector spaces and let $b \in L(X, Y)$. Its transpose $b^{\mathrm{t}} \in L\left(Y^{\#}, X^{\#}\right)$ is defined by

$$
\left\langle b^{\mathrm{t}} z, f\right\rangle=\langle z, b f\rangle, \quad z \in Y^{\#}, \quad f \in X .
$$

Let $V, W$ be complex vector spaces and let $a \in L(V, W)$. The dual operator $a^{*} \in$ $L\left(\bar{W}^{*}, \bar{V}^{*}\right)$ is defined by

$$
\left\langle a^{*} z, v\right\rangle=\langle z, a v\rangle, \quad z \in \bar{W}^{*}, \quad v \in V .
$$

Note that we have not assumed so far that the vector spaces are endowed with some topology. In particular, the dual operator is not to be confused with the Hilbert space adjoint.

Definition 1.2.2. Let $(X, \beta)$ be a symplectic space (resp. orthogonal space). The symplectic group $S p(X, \beta)$ (resp. orthogonal group $O(X, \beta)$ ) consists of all bijective $u \in L(X)$ s.t.

$$
u^{\mathrm{t}} \beta u=\beta .
$$

Let $(V, \alpha)$ be a pre-unitary space. The unitary group $U(V, \alpha)$ consists of all bijective $u \in L(V)$ s.t.

$$
u^{*} \alpha u=\alpha .
$$

Note that in the sesquilinear notation, (1.2.2) means that

$$
\alpha(u v, u w)=\alpha(v, w), \quad v, w \in V .
$$

Observe also that

$$
U(V, \alpha)=O\left(V_{\mathbb{R}}, \operatorname{Re} \alpha\right) \cap S p\left(V_{\mathbb{R}}, \operatorname{Im} \alpha\right) .
$$

ExAmple 1 . Let $(V, \alpha)$ be a pre-unitary space and consider the hermitian form on $V \oplus V$ defined by

$$
q(v, w):=\alpha\left(v_{0}, w_{1}\right)+\alpha\left(v_{1}, w_{0}\right), \quad v=\left(\begin{array}{l}
v_{0} \\
v_{1}
\end{array}\right), w=\left(\begin{array}{l}
w_{0} \\
w_{1}
\end{array}\right) \in V \oplus V .
$$


Then $(V \oplus V, q)$ is a pre-unitary space. If now $r, s, t \in L(V)$ are such that $r^{*}=-r$, $s^{*}=-s$ and $t$ is bijective then the operators
(1) $\left(\begin{array}{ll}\mathbf{1} & 0 \\ r & \mathbf{1}\end{array}\right)$,
(2) $\left(\begin{array}{ll}\mathbf{1} & s \\ 0 & \mathbf{1}\end{array}\right)$ and
(3) $\left(\begin{array}{cc}t^{*} & 0 \\ 0 & t^{-1}\end{array}\right)$

belong to the unitary group $U(V \oplus V, q)$. Moreover, one can show that if

$$
u=\left(\begin{array}{cc}
a & b \\
c & d
\end{array}\right) \in L(V \oplus V)
$$

is an element of $U(V \oplus V, q)$ such that $a \in L(V)$ is bijective then it can be represented as the composition of three operators of the form $(1),(2),(3)$.

Pre-unitary spaces of the form $(V \oplus V, q)$ arise naturally in many problems. For instance, if $V$ is finite dimensional then $q$ is simply the complex equivalent of the canonical symplectic form. We will also encounter spaces of this kind in the context of solutions of the Klein-Gordon equation.

\subsection{Representations of CARs and CCRs}

In what follows, $\mathcal{H}$ is a Hilbert space and we denote $\mathcal{B}(\mathcal{H}), \mathcal{U}(\mathcal{H}), \mathcal{C}(\mathcal{H})$ respectively the set of bounded, unitary and closed operators acting in $\mathcal{H}$. The identity in $\mathcal{B}(\mathcal{H})$ will be denoted 1 .

1.3.1. Neutral fermions. Let $(X, \nu)$ be an orthogonal space and let $\mathcal{H}$ be a Hilbert space. If $A, B \in \mathcal{B}(\mathcal{H})$ then their anti-commutator is denoted $\{A, B\}:=$ $A B+B A$.

Definition 1.3.1. A $C A R$ representation over $(X, \nu)$ in $\mathcal{H}$ is a map

$$
X \ni f \mapsto \phi^{\pi}(f) \in \mathcal{B}(\mathcal{H})
$$

with values in self-adjoint operators, which satisfies

$$
\left\{\phi^{\pi}(f), \phi^{\pi}(g)\right\}=2 \nu(f, g) \mathbf{1}, \quad f, g \in X .
$$

The superscript ${ }^{\pi}$ in the notation above has no mathematical meaning for the moment, it is however useful when one wants to distinguish between different CAR representations. The notation $\phi(f)$ will be reserved for elements of the CAR $C^{*}$ algebra later on.

Definition 1.3.2. Let $\mathcal{H}_{1}, \mathcal{H}_{2}$ be Hilbert spaces. One says that two CAR representations

$$
X \ni f \mapsto \phi^{\pi_{1}}(f) \in \mathcal{B}\left(\mathcal{H}_{1}\right), \quad X \ni f \mapsto \phi^{\pi_{2}}(f) \in \mathcal{B}\left(\mathcal{H}_{2}\right)
$$

over the same orthogonal space $(X, \nu)$ are unitary equivalent if there exists a unitary operator $U \in \mathcal{U}\left(\mathcal{H}_{1}, \mathcal{H}_{2}\right)$ s.t.

$$
U \phi^{\pi_{1}}(f)=\phi^{\pi_{2}}(f) U, \quad f \in X .
$$

The theory of CAR representations over an infinite-dimentional space $X$ is highly non-trivial in the sense that in general there exist many unitary non-equivalent representations. 
1.3.2. Charged fermions. Let $(V, q)$ be a unitary space, that is a complex vector space equipped with a positive sesquilinear form $q$. The complex structure on $V$ will be denoted by $\mathrm{j}$ to distinguish it from the complex number $\mathrm{i} \in \mathbb{C}$.

Let $\mathcal{H}$ be a Hilbert space.

Definition 1.3.3. A charged $C A R$ representation over $(V, q)$ is a map

$$
V \ni v \mapsto \psi^{\pi}(v) \in \mathcal{B}(\mathcal{H})
$$

which satisfies

$$
\begin{aligned}
& \left\{\psi^{\pi *}(v), \psi^{\pi *}(w)\right\}=\left\{\psi^{\pi}(v), \psi^{\pi}(w)\right\}=0 \\
& \left\{\psi^{\pi}(v), \psi^{\pi *}(w)\right\}=q(v, w) \mathbf{1}, \quad v, w \in V .
\end{aligned}
$$

A charged CAR representation over $V$ induces a CAR representation over $V_{\mathbb{R}}$. Namely, let $v \mapsto \psi^{\pi}(v)$ be a charged CAR representation over $(V, q)$ and set

$$
X:=V_{\mathbb{R}}, \quad \nu:=\frac{1}{2} \operatorname{Re} q, \quad \phi^{\pi}(v):=\frac{1}{2}\left(\psi^{\pi}(v)+\psi^{\pi *}(v)\right) .
$$

Then $X \ni f \mapsto \phi^{\pi}(f)$ is a CAR representation over $(X, \nu)$.

1.3.3. Neutral bosons. Let $(X, \sigma)$ be a real symplectic space. The implementation of the CCRs require more care than in the fermionic case, because the field operators are necessarely unbounded. It is possible to work with bounded operators by considering an exponentiated version of the CCRs, called the Weyl canonical commutation relations.

Definition 1.3.4. A $C C R$ representation over $(X, \sigma)$ in $\mathcal{H}$ is a map

$$
X \ni f \mapsto W^{\pi}(f) \in \mathcal{U}(\mathcal{H})
$$

which satisfies

$$
W^{\pi}(f) W^{\pi}(g)=\mathrm{e}^{-\frac{1}{2} \sigma(f, g)} W^{\pi}(f+g), \quad f, g \in X .
$$

An obvious consequence of (1.3.3) are the equalities

$$
W^{\pi *}(f)=W^{\pi}(-f), \quad W^{\pi}\left(t_{1} f\right) W^{\pi}\left(t_{2} f\right)=W^{\pi}\left(\left(t_{1}+t_{2}\right) f\right), \quad f \in X, \quad t_{1}, t_{2} \in \mathbb{R} .
$$

Definition 1.3.5. Let $\mathcal{H}_{1}, \mathcal{H}_{2}$ be Hilbert spaces. One says that two CCR representations

$$
X \ni f \mapsto W^{\pi_{1}}(f) \in \mathcal{U}\left(\mathcal{H}_{1}\right), \quad X \ni f \mapsto W^{\pi_{2}}(f) \in \mathcal{U}\left(\mathcal{H}_{2}\right)
$$

over the same pre-symplectic space $(X, \sigma)$ are unitary equivalent if there exists a unitary operator $U \in \mathcal{U}\left(\mathcal{H}_{1}, \mathcal{H}_{2}\right)$ s.t.

$$
U W^{\pi_{1}}(f)=W^{\pi_{2}}(f) U, \quad f \in X .
$$

Definition 1.3.6. A CCR representation is called regular if for any $f \in X$ the map

$$
\mathbb{R} \ni t \mapsto W^{\pi}(t f) \in \mathcal{U}(\mathcal{H})
$$

is strongly continuous. 
Given a regular CCR representation, the field operators $\phi^{\pi}(f)$ are defined as generators of the one-parameter strongly continuous group of unitaries (1.3.4), i.e.,

$$
\phi^{\pi}(f):=-\left.\mathrm{i} \frac{d}{d t} W^{\pi}(t f)\right|_{t=0} \in \mathcal{C}(\mathcal{H}) .
$$

For an operator $A \in \mathcal{C}(\mathcal{H})$, we denote $\operatorname{Dom} A$ its domain. The field operators have the following basic properties.

Proposition 1.3.7. Let $X \ni f \mapsto \mathrm{e}^{\mathrm{i} \phi^{\pi}(f)}$ be a CCR representation. Then for all $f, g \in X$

(1) $\phi^{\pi}(t x)=t \phi^{\pi}(f), \quad t \in \mathbb{R}$

(2) $\operatorname{Dom} \phi^{\pi}(f) \cap \operatorname{Dom} \phi^{\pi}(g) \subset \operatorname{Dom} \phi^{\pi}(f+g)$;

(3) $\phi^{\pi}(f+g)=\phi^{\pi}(f)+\phi^{\pi}(g)$ on $\operatorname{Dom} \phi^{\pi}(f) \cap \operatorname{Dom} \phi^{\pi}(g)$;

(4) as a quadratic form on $\operatorname{Dom} \phi^{\pi}(f) \cap \operatorname{Dom} \phi^{\pi}(g)$,

$$
\left[\phi^{\pi}(f), \phi^{\pi}(g)\right]=\mathrm{i} \sigma(f, g) 1 .
$$

In order to avoid cumbersome assumptions on the domains in formulae which involve products of operators, it is often useful to work with the subspace

$$
\mathcal{H}^{\infty, \pi}:=\bigcap_{n=1}^{\infty} \bigcap_{f_{1}, \ldots, f_{n} \in X} \operatorname{Dom} \phi^{\pi}\left(f_{1}\right) \cdots \phi^{\pi}\left(f_{n}\right) \subset \mathcal{H},
$$

where the domain of the product of unbounded operators is defined by

$$
\operatorname{Dom} A B=\{h \in \operatorname{Dom} B: B h \in \operatorname{Dom} A\}, \quad A, B \in \mathcal{C}(\mathcal{H}) .
$$

Obviously, $\phi^{\pi}(f) \mathcal{H}^{\infty, \pi} \subset \mathcal{H}^{\infty, \pi}$. One can show that if $X$ is finite dimensional then $\mathcal{H}^{\infty, \pi}$ is dense in $\mathcal{H}$; this will also be the case in the infinite-dimensional examples we will be interested in later on.

1.3.4. Charged bosons. Let $(V, q)$ be a pre-unitary space and let $\mathcal{H}$ be a Hilbert space. Representations of the CCRs in the charged case can be defined via a reduction to the neutral case.

DeFinition 1.3.8. One says that a map

$$
V \ni v \mapsto \psi^{\pi}(v) \in \mathcal{C}(\mathcal{H})
$$

is a charged $C C R$ representation over $(V, q)$ if there exists a regular neutral CCR representation over $\left(V_{\mathbb{R}}, \operatorname{Im} q\right)$

$$
V \ni v \mapsto W^{\pi}(v)=\mathrm{e}^{\mathrm{i} \phi^{\pi}(v)} \in \mathcal{U}(\mathcal{H})
$$

such that

$$
\psi^{\pi}(v)=\frac{1}{\sqrt{2}}\left(\phi^{\pi}(v)+\mathrm{i} \phi^{\pi}(\mathrm{j} v)\right), \quad v \in V .
$$

Observe that the 'neutral' field operators $\phi^{\pi}(v)$ can be recovered from the operators $\psi^{\pi}(v)$ (called charged field operators). Indeed, using that $\mathrm{j}^{2}=\mathbf{- 1}$ we get from 
(1.3.6) the relation

$$
\phi^{\pi}(v)=\frac{1}{\sqrt{2}}\left(\psi^{\pi}(v)+\psi^{\pi *}(v)\right) .
$$

Up to technicalities due to unboundedness of the operators, the map $V \ni v \mapsto$ $\psi^{\pi *}(v)$ is linear, the map $V \ni v \mapsto \psi^{\pi}(v)$ is anti-linear, and the charged field operators $\psi^{\pi}(v)$ satisfy the CCRs.

Proposition 1.3.9. Let $V \ni v \mapsto \psi^{\pi}(v)$ be a charged CCR representation. Then for all $v, w \in V$,

(1) $\psi^{\pi}(\lambda v)=\bar{\lambda} \psi^{\pi}(v), \quad \lambda \in \mathbb{C}$

(2) $\operatorname{Dom} \psi^{\pi}(v) \cap \operatorname{Dom} \psi^{\pi}(w) \subset \operatorname{Dom} \psi^{\pi}(v+w)$;

(3) $\psi^{\pi}(v+w)=\psi^{\pi}(v)+\psi^{\pi}(w)$ on $\operatorname{Dom} \psi^{\pi}(v) \cap \operatorname{Dom} \psi^{\pi}(w)$;

(4) as quadratic forms on $\operatorname{Dom} \psi^{\pi}(v) \cap \operatorname{Dom} \psi^{\pi}(w)$,

$$
\begin{aligned}
& {\left[\psi^{\pi}(v), \psi^{\pi}(w)\right]=\left[\psi^{\pi *}(v), \psi^{\pi *}(w)\right]=0,} \\
& {\left[\psi^{\pi}(v), \psi^{\pi *}(w)\right]=q(v, w) \mathbf{1}}
\end{aligned}
$$

\subsection{CAR and CCR $C^{*}$-algebras}

Let $\mathfrak{A}$ be a $C^{*}$-algebra. A state $\omega$ is a positive continuous linear functional on $\mathfrak{A}$ of norm one. Here 'positive' means that $\omega\left(a^{*} a\right) \geq 0$ for all $a \in \mathfrak{A}$. The set of all states on $\mathfrak{A}$ is a convex subset of the algebraic dual $\mathfrak{A}^{*}$, and it is closed in the weak-* topology.

Definition 1.4.1. A state on $\mathfrak{A}$ is pure if it is an extremal point in the set of all states on $\mathfrak{A}$.

In other words, $\omega$ is pure if it cannot be written as a convex sum of two states $\omega_{1}$, $\omega_{2}$, both of which are not equal $\omega$.

Theorem 1.4.2. Let $\omega$ be a state on $\mathfrak{A}$. Then there exists a triple $\left(\mathcal{H}_{\omega}, \pi_{\omega}, \Omega_{\omega}\right)$ which consists of:

- a Hilbert space $\mathcal{H}_{\omega}$,

- a $*$-representation $\pi_{\omega}$ in $\mathcal{H}_{\omega}$, i.e., a $*$-homomorphism of $\mathfrak{A}$ into $\mathcal{B}\left(\mathcal{H}_{\omega}\right)$,

- a cyclic vector $\Omega_{\omega}$ (i.e., an element of $\mathcal{H}_{\omega}$ s.t. $\pi_{\omega}(\mathfrak{A}) \Omega_{\omega}$ is dense in $\mathcal{H}$ ), which satisfies

$$
\omega(a)=\left(\Omega_{\omega} \mid \pi_{\omega}(a) \Omega_{\omega}\right), \quad a \in \mathfrak{A} .
$$

Moreover, if $\left(\tilde{\mathcal{H}}_{\omega}, \tilde{\pi}_{\omega}, \tilde{\Omega}_{\omega}\right)$ is another such triple then there exists $U \in \mathcal{U}\left(\mathcal{H}_{\omega}, \tilde{\mathcal{H}}_{\omega}\right)$ such that

$$
\begin{aligned}
& U \pi_{\omega}(a)=\tilde{\pi}_{\omega}(a) U, \quad a \in \mathfrak{A}, \\
& U \Omega_{\omega}=\tilde{\Omega}_{\omega} .
\end{aligned}
$$

The triple $\left(\mathcal{H}_{\omega}, \pi_{\omega}, \Omega_{\omega}\right)$ is called the GNS representation associated with the state $\omega$.

Suppose now that $\mathfrak{A}$ is a $C^{*}$-algebra with unit, equipped with a strongly continuous 
group $\mathbb{R} \ni t \mapsto \alpha_{t}$ of $*$-isomorphisms. One says that $\omega$ is a ground state w.r.t. $\alpha_{t}$ if $\omega \circ \alpha_{t}=\omega$ for all $t \in \mathbb{R}$ and there exists a strongly continuous group of unitaries $\mathbb{R} \ni t \mapsto U_{t} \in \mathcal{U}\left(\mathcal{H}_{\omega}\right)$ with positive generator, s.t. $U_{t} \Omega_{\omega}=\Omega_{\omega}$ and

$$
\pi_{\omega}\left(\alpha_{t}(a)\right)=U_{t} \pi_{\omega}(a) U_{t}^{-1}, \quad a \in \mathfrak{A}, t \in \mathbb{R} .
$$

If, additionally, the eigenspace of the generator of $U_{t}$ corresponding to the eigenvalue 0 is spanned by $\Omega_{\omega}$, then $\omega$ is pure [Bor66].

1.4.1. CAR $C^{*}$-algebra. Let $(X, \nu)$ be an orthogonal space. Let $\operatorname{CAR}_{\text {alg }}(X, \nu)$ be the *-algebra generated by elements $\phi(f)$ for $f \in X$, subject to relations

$$
\begin{aligned}
\phi(\lambda x)=\lambda \phi(f), \lambda \in \mathbb{R}, \quad \phi(f+g)=\phi(f)+\phi(g), \\
\phi^{*}(f)=\phi(f), \quad \phi(f) \phi(g)-\phi(g) \phi(f)=2 \nu(f, g) \mathbf{1}, \quad f, g \in X .
\end{aligned}
$$

It can be proved that there exists a unique $C^{*}$-norm on $\operatorname{CAR}_{\text {alg }}(X, \nu)$.

Definition 1.4.3. The CAR $C^{*}$-algebra, denoted $\operatorname{CAR}(X, \nu)$, is the completion of $\operatorname{CAR}_{\text {alg }}(X, \nu)$ with respect to the above norm.

If $\omega$ is a state on $\operatorname{CAR}(X, \nu)$ and $(\mathcal{H}, \pi, \Omega)$ is the associated GNS representation, then

$$
X \ni f \mapsto \phi^{\pi}(f):=\pi(\phi(f))
$$

is a CAR representation. The converse statement can be formulated as follows.

Proposition 1.4.4. Let $X \ni f \mapsto \phi^{\pi}(f) \in \mathcal{B}(\mathcal{H})$ be a CAR representation. Then there exists a unique $*$-homomorphism $\pi: \operatorname{CAR}(X, \nu) \rightarrow \mathcal{B}(\mathcal{H})$ such that

$$
\pi(\phi(f))=\phi^{\pi}(f), \quad f \in X .
$$

1.4.2. Weyl CCR $C^{*}$-algebra. In the bosonic case one can choose between several different $C^{*}$-algebras which encode the CCRs. In the literature on QFT on curved spacetimes, one uses mostly the so-called Weyl CCR $C^{*}$-algebra. It is constructed as follows.

Let $(X, \sigma)$ be a pre-symplectic space. Let $\mathrm{CCR}_{\text {alg }}(X, \sigma)$ be the $*$-algebra generated by elements $W(f)$ for all $f \in X$, subject to relations

$$
W(f)^{*}=W(-f), \quad W(f) W(g)=\mathrm{e}^{-\frac{1}{2} \sigma(f, g)}, \quad f, g \in X
$$

Observe that if $X \ni f \mapsto W^{\pi}(f) \in \mathcal{U}(\mathcal{H})$ is a CCR representation in a Hilbert space $\mathcal{H}$, then there exists a unique unital $*$-isomorphism $\pi: \mathrm{CCR}_{\text {alg }}(X, \sigma) \rightarrow \mathcal{B}(\mathcal{H})$ such that

$$
\pi(W(f))=W^{\pi}(f), \quad f \in X .
$$

This way, the class of all CCR representations over $(X, \sigma)$ can be identified with a set of $*$-isomorphisms, which we will denote $\mathcal{R}(X, \sigma)$ in what follows. A well-known fact says that the set $\mathcal{R}(X, \sigma)$ is not empty (note that we take into account all CCR representations, not only regular ones). 
This allows us to introduce a norm $\|\cdot\|$ on $\operatorname{CCR}_{\text {alg }}(X, \sigma)$ by

$$
\|a\|:=\sup \{\|\pi(a)\|, \pi \in \mathcal{R}(X, \sigma)\}, \quad a \in \mathrm{CCR}_{\text {alg }}(X, \sigma) .
$$

Definition 1.4.5. The Weyl CCR algebra, denoted $\operatorname{CCR}(X, \sigma)$, is the completion of $\mathrm{CCR}_{\text {alg }}(X, \sigma)$ with respect to the norm $\|\cdot\|$.

The $*$-algebra $\operatorname{CCR}(X, \sigma)$ is a $C^{*}$-algebra indeed. Let us remark that so far we have not assumed that $(X, \sigma)$ is a symplectic space, i.e., we allowed $\sigma$ to be a degenerate form. It has been recently suggested that such degenerate forms arise naturally in quantization of gauge fields on curved spacetimes [SDH12]. In the examples we will consider, however, we will always be in the non-degenerate case. Then the $C^{*}$ algebra $\operatorname{CCR}(X, \sigma)$ enjoys special properties, for instance it is simple, i.e., it possesses no non-trivial closed two-sided ideals.

If $\omega$ is a state on $\operatorname{CCR}(X, \sigma)$ and $(\mathcal{H}, \pi, \Omega)$ is the associated GNS representation, then

$$
X \ni f \mapsto W^{\pi}(f):=\pi(W(f)) \in \mathcal{B}(\mathcal{H})
$$

is a CCR representation. The converse statement can be formulated as follows (note that it is not completely trivial because it involves continuity of $\pi$ ).

Proposition 1.4.6. Let $X \ni f \mapsto W^{\pi}(f) \in \mathcal{U}(\mathcal{H})$ be a regular CCR representation. Then there exists a unique $*$-homomorphism $\pi: \operatorname{CCR}(X, \sigma) \rightarrow \mathcal{B}(\mathcal{H})$ s.t.

$$
\pi(W(f))=W^{\pi}(f), \quad f \in X .
$$

One can argue that different other choices of $C^{*}$-algebras are more useful in the context of gauge-invariant observables, an example is the resolvent algebra proposed relatively recently by Buchholz and Grundling [BG08].

\subsection{Quasi-free states}

1.5.1. Neutral fermions. Let $(X, \nu)$ be an orthogonal space. The simplest class of states on $\operatorname{CAR}(X, \nu)$ are the so-called quasi-free states, which are the ones fully determined by their evaluation on elements of the form $\phi(f) \phi(g), f, g \in X$ (and their evaluation on $\phi(f), f \in X$ is zero). They can be rigorously defined as follows.

Definition 1.5.1. A state $\omega$ on $\operatorname{CAR}(X, \nu)$ is called a (fermionic, neutral) quasi-free state if there exists an anti-symmetric form $\beta$ on $X$, called the covariance of $\omega$ s.t.

$$
\omega(\phi(f) \phi(g))=\nu(f, g)+\frac{\mathrm{i}}{2} \beta(f, g), \quad f, g \in X .
$$

Anti-symmetry of $\beta$ entails that a quasi-free state $\omega$ is indeed fully determined by (1.5.1), as the next proposition illustrates.

Proposition 1.5.2. Let $\omega$ be a quasi-free state on $\operatorname{CAR}(X, \nu)$. Then

$$
\begin{aligned}
\omega\left(\phi\left(f_{1}\right) \ldots \phi\left(f_{2 n-1}\right)\right) & =0, \\
\omega\left(\phi\left(f_{1}\right) \ldots \phi\left(f_{2 n}\right)\right) & =\sum_{s \in P_{2 n}} \operatorname{sgn}(s) \prod_{j=1}^{n} \omega\left(\phi\left(f_{s(2 j-1)}\right) \phi\left(f_{s(2 j)}\right)\right),
\end{aligned}
$$


for all $n \in \mathbb{N}, f_{1}, \ldots, f_{2 n} \in X$, where

$$
P_{2 n}=\left\{s \in S_{2 n}: s(2 i-1)<\max \{s(2 i), s(2 i+1)\}, 1 \leq i \leq n\right\}
$$

and $S_{2 n}$ is the set of even permutations of $\{1, \ldots, 2 n\}$.

A quasi-free state is uniquely determined by its covariance $\beta$. Our first goal will be to set up an analogous equivalence between quasi-free states and their two-point function, which are a complexified version of the symmetric form (1.5.1).

DEFINITION 1.5.3. The two-point function of a (fermionic, neutral) quasi-free state on $\operatorname{CAR}(X, \nu)$ (with covariance $\beta$ ) is the sesquilinear hermitian form

$$
\lambda:=\nu_{\mathbb{C}}+\frac{\mathrm{i}}{2} \beta_{\mathbb{C}} \in L\left(\mathbb{C} X, \overline{\mathbb{C} X}^{*}\right),
$$

In order to have a closer analogy to the charged case later on, it is useful to introduce at once the positive sesquilinear form

$$
q:=2 \nu_{\mathbb{C}} \in L\left(\mathbb{C} X, \overline{\mathbb{C} X}^{*}\right) .
$$

Proposition 1.5.4. Let $\beta \in L\left(X, X^{\#}\right)$ be an anti-symmetric form and let $\lambda, q$ be defined by $(1.5 .2),(1.5 .3)$. Then the following are equivalent:

(1) $\beta$ is the covariance of a quasi-free state on $\operatorname{CAR}(X, \nu)$;

(2) $|\beta(f, g)| \leq 2(\nu(f, f))^{\frac{1}{2}}(\nu(g, g))^{\frac{1}{2}}$ for all $f, g \in X$;

(3) $\lambda \geq 0$ on $\mathbb{C} X$

(4) $\lambda \geq 0$ and $\lambda \leq q$ on $\mathbb{C} X$.

Let us stress that although conditions (3) and (4) are equivalent if $\lambda=\nu_{\mathbb{C}}+\frac{i}{2} \beta_{\mathbb{C}}$ for some symmetric $\nu$ and anti-symmetric $\beta$, this is not necessarily true for a generic sesquilinear form $\lambda \in L\left(\mathbb{C} X, \overline{\mathbb{C}}^{*}\right)$. In the statement of the corollary below, which can be derived as a direct consequence of Proposition 1.5.4 and Lemma 1.1.4, we avoid making a detour via real forms.

Corollary 1.5.5. Let $\lambda \in L\left(\mathbb{C} X, \overline{\mathbb{C} X}^{*}\right)$. Then the following are equivalent:

(1) $\lambda$ is the two-point function of a quasi-free state on $\operatorname{CAR}(X, \nu)$;

(2) $\lambda \geq 0$ and $\lambda \leq q$.

It is well known that the subclass of pure quasi-free states can be characterized as follows in terms of their covariances $\beta$.

Proposition 1.5.6. Let $\beta \in L\left(X, X^{\#}\right)$ be anti-symmetric. The following are equivalent:

(1) $\beta$ is the covariance of a pure quasi-free state on $\operatorname{CAR}(X, \nu)$;

(2) there exists $\mathrm{j} \in S p(X, \beta)$ s.t. $\mathrm{j}^{2}=-\mathbf{1}$ and $2 \nu=\beta \mathrm{j}$.

Using Proposition 1.5.4 we conclude immediately that if $\beta$ is the covariance of a quasi-free state on $\operatorname{CAR}(X, \nu)$ then the same is true for $r^{\mathrm{t}} \beta r$ for any $r \in O(X, \nu)$. Moreover, from 1.5.6 we see that if $\beta$ was the covariance of a pure state, then so is $r^{\mathrm{t}} \beta r$. A converse statement holds true in the case when $X$ is complete. More generally, we take the completion of $X$ w.r.t. $\nu$ and denote it $\mathcal{X}$. We can then 
consider covariances $\beta \in L\left(X, X^{\#}\right)$ which extend to bounded operators from $\mathcal{X}$ to the topological dual $\mathcal{X}^{\#} \approx \mathcal{X}$, i.e., elements of $\mathcal{B}(\mathcal{X})$.

Proposition 1.5.7. Let $(X, \nu)$ be an orthogonal space and let $\mathcal{X}$ be the completion of $X$ w.r.t. the norm induced by $\nu$. Let $\beta_{1} \in \mathcal{B}(\mathcal{X}), \beta_{2} \in \mathcal{B}(\mathcal{X})$ be covariances of two pure quasi-free states on $\operatorname{CAR}(X, \nu)$. Then there exists an operator $r \in$ $\mathcal{B}(\mathcal{X}) \cap O(X, \nu)$ s.t. $\beta_{2}=r^{\mathrm{t}} \beta_{1} r$, where $r^{\mathrm{t}} \in \mathcal{B}(\mathcal{X})$ stands for the Hilbert-space adjoint.

Proof. See, e.g., [DG13].

1.5.2. Charged fermions. Let $(V, q)$ be a unitary space.

According to the discussion in Sect. 1.1, $\left(V_{\mathbb{R}}, \frac{1}{2} \mathrm{Re} q\right)$ is a real symplectic space. Observe that if $\mathrm{j}$ denotes the complex structure on $V$ then clearly $\mathrm{j} \in O\left(V_{\mathbb{R}}, \frac{1}{2} \operatorname{Re} q\right)$.

For coherence of notation we will denote the $\mathrm{CAR} C^{*}$-algebra

$$
\operatorname{CAR}(V, q):=\operatorname{CAR}\left(V_{\mathbb{R}}, \frac{1}{2} \operatorname{Re} q\right) .
$$

By definition, (fermionic) charged quasi-free states are simply quasi-free states on $\operatorname{CAR}(V, q)$ in the sense of the previous subsection.

Again, in the charged case physical states are required to satisfy a gauge-invariance condition.

Definition 1.5.8. A state $\omega$ on $\operatorname{CAR}(V, q)$ is called gauge-invariant if

$$
\omega(\phi(v))=\omega\left(\phi\left(\mathrm{e}^{\mathrm{j} \theta} v\right)\right), \quad 0 \leq \theta<2 \pi, \quad v \in V .
$$

If $\omega$ is a quasi-free state with covariance $\beta$ then gauge-invariance of $\omega$ is equivalent to

$$
\mathrm{j} \in O\left(V_{\mathbb{R}}, \beta\right) .
$$

In order to implement the algebraic approach in the charge case, let us introduce the abstract charged fields:

$$
\psi(v):=\frac{1}{2}(\phi(v)+\mathrm{i} \phi(\mathrm{j} v)) \in \operatorname{CAR}(V, q), \quad v \in V .
$$

If $\omega$ is a state on $\operatorname{CAR}(V, q)$ and $(\mathcal{H}, \pi, \omega)$ is the associated GNS representation then

$$
V \ni v \mapsto \psi^{\pi}(v):=\omega(\psi(v)) \in \mathcal{B}(\mathcal{H})
$$

is a charged CAR representation.

The next proposition characterizes gauge-invariant quasi-free states.

Proposition 1.5.9. A state $\omega$ on $\operatorname{CAR}(V, q)$ is gauge-invariant iff

$$
\omega\left(\psi^{*}\left(v_{1}\right) \cdots \psi^{*}\left(v_{n}\right) \psi\left(w_{1}\right) \ldots \psi\left(w_{m}\right)\right)=0, \quad n \neq m
$$

for all $v_{1}, \ldots, v_{n}, w_{1}, \ldots, w_{m} \in V$. It is also quasi-free if in addition

$$
\omega\left(\psi^{*}\left(v_{1}\right) \cdots \psi^{*}\left(v_{n}\right) \psi\left(w_{1}\right) \ldots \psi\left(w_{n}\right)\right)=\sum_{s \in S_{n}} \operatorname{sgn}(s) \prod_{j=1}^{n} \omega\left(\psi^{*}\left(v_{j}\right) \psi\left(w_{s(j)}\right)\right),
$$


for all $n \in N, v_{1}, \ldots, v_{n}, w_{1}, \ldots, w_{n} \in V$, where $S_{n}$ denotes the set of even permutations of $\{1, \ldots, n\}$.

If $\omega$ is a gauge-invariant quasi-free state on $\operatorname{CAR}(V, q)$ then we also have

$$
\omega(\psi(v) \psi(w))=\omega\left(\psi^{*}(v) \psi^{*}(w)\right)=0, \quad v, w \in V .
$$

Thus, such state is uniquely determined by the expression $\omega\left(\psi(v) \psi^{*}(w)\right), v, w \in V$.

DeFinition 1.5.10. The two-point function of a (fermionic, charged) gauge-invariant quasi-free state $\omega$ is the hermitian form $\lambda \in L\left(V, \bar{V}^{*}\right)$ given by

$$
\lambda(v, w):=\omega\left(\psi(v) \psi^{*}(w)\right), \quad v, w \in V .
$$

The charge density is the hermitian form

$$
c(v, w):=\omega\left(\psi^{*}(w) \psi(v)\right), \quad v, w \in V .
$$

We now derive necessary and sufficient conditions for a sesquilinear form $\lambda$ to be the two-point function of a gauge-invariant, quasi-free state. The result turns out to be completely analogous to what we had in the neutral case (cf. Proposition 1.5.6).

Proposition 1.5.11. Let $\lambda \in L\left(V, \bar{V}^{*}\right)$. Then the following are equivalent:

(1) $\lambda$ is the two-point function of a gauge-invariant quasi-free state on $\operatorname{CAR}(V, q)$;

(2) $\lambda \geq 0$ and $\lambda \leq q$.

Proof. Using $\phi(v)=\frac{1}{2}\left(\psi(v)+\psi^{*}(v)\right)$, (1.5.6) and the CARs, we compute

$$
\begin{aligned}
\omega(\phi(v) \phi(w)) & =\frac{1}{4} \omega\left(\psi(v) \psi^{*}(w)\right)+\frac{1}{4} \omega\left(\psi^{*}(v) \psi(w)\right) \\
& =\frac{1}{4} \omega\left(\psi(v) \psi^{*}(w)\right)-\frac{1}{4} \omega\left(\psi(w) \psi^{*}(v)\right)+\frac{1}{4} q(w, v) \\
& =\frac{1}{2} \operatorname{Im} \lambda(v, w)+\frac{1}{4} q(w, v)=\frac{1}{4} \operatorname{Re} q(v, w)+\frac{i}{2} \operatorname{Im}\left(\lambda(v, w)-\frac{1}{2} q(v, w)\right),
\end{aligned}
$$

hence $\beta=\operatorname{Im}\left(\lambda-\frac{1}{2} q\right)$ and we can express $\tilde{\lambda}:=\nu_{\mathbb{C}}+\frac{\mathrm{i}}{2} \beta_{\mathbb{C}}$ in terms of $q$ and the complexifiaction of $\operatorname{Im} \lambda$. Since $\omega$ is gauge-invariant we have

$$
\mathrm{j} \in S p\left(V_{\mathbb{R}}, \beta\right) \cap O\left(V_{\mathbb{R}}, \frac{1}{2} \operatorname{Re} q\right)=S p\left(V_{\mathbb{R}}, \beta\right) \cap S p\left(V_{\mathbb{R}}, \frac{1}{2} \operatorname{Im} q\right) .
$$

From this fact we deduce the equivalence of the conditions $\tilde{\lambda} \geq 0, \tilde{\lambda} \leq q$ and $\lambda \geq 0$, $\lambda \leq q$

Remark 1.5.12. Let $\mathcal{V}$ be the completion of $V$ with respect to the norm induced by $q$. If a two-point function $\lambda \in L\left(V, \bar{V}^{*}\right)$ extends to a bounded operator $\lambda \in$ $\mathcal{B}\left(\mathcal{V}, \overline{\mathcal{V}}^{*}\right)$ (where $\overline{\mathcal{V}}^{*}$ stands for the dual in the sense of Banach spaces), then under the identification $\mathcal{B}\left(\mathcal{V}, \overline{\mathcal{V}}^{*}\right) \approx \mathcal{B}(\mathcal{V})$ condition (2) becomes

$$
0 \leq \lambda \leq \mathbf{1}
$$

in the sense of positivity of operators on the Hilbert space $\mathcal{V}$. This is the condition which appears in most of the literature.

Proposition 1.5.13. Let $\lambda \in L\left(V, \bar{V}^{*}\right)$ be hermitian. Then the following are equivalent:

(1) $\lambda$ is the two-point function of a pure gauge-invariant quasi-free state on 
$\operatorname{CAR}(V, q)$

(2) there exists an involution $\kappa \in U(V, q)$ s.t. $q \kappa \geq 0$ and $\lambda=\frac{1}{2} q(\mathbf{1}-\kappa)$;

(3) $\lambda \leq \frac{1}{2} q$ and $\lambda q^{-1} \lambda=\lambda$.

Proof. By Proposition 1.5.20 the state $\omega$ is pure iff there exists an anti-involution $\mathrm{j} \in S p\left(V_{\mathbb{R}}, \operatorname{Im} q\right)$ such that

$$
\operatorname{Re} q=\beta \mathbf{j} .
$$

Since $\mathrm{j} \in S p\left(V_{\mathbb{R}}, \beta\right) \cap S p\left(V_{\mathbb{R}}, \frac{1}{2} \operatorname{Im} q\right)$ we obtain that $\mathrm{j} \in U(V, q)$. Complexifying (1.5.7) we then get that $q=2 \mathrm{i}(\nu-\lambda)$. Setting $\kappa=-\mathrm{ij}$ we see that $\kappa \in U(V, q)$ and $\lambda=\frac{1}{2} q(\mathbf{1}-\kappa)$. Therefore (1) is equivalent to

$$
\text { (4) } \lambda \geq 0, \lambda \geq q, \lambda=\frac{1}{2} q(\mathbf{1}+\kappa), \kappa^{2}=\mathbf{1}, \kappa \in U(V, q) \text {. }
$$

(4) clearly implies (2). Let us prove the converse implication. Set $p_{ \pm}:=\frac{1}{2}(\kappa \pm 1)$. Clearly $p_{ \pm}$are projections with $p_{ \pm}^{*} q=q p_{ \pm}, \kappa p_{ \pm}= \pm p_{ \pm}$, and

$$
\lambda \geq 0, \lambda \leq q \Leftrightarrow \pm q p_{ \pm} \geq 0 .
$$

Now we have

$$
q p_{ \pm}=q p_{ \pm}^{2}=p_{ \pm}^{*} q p_{ \pm}= \pm p_{ \pm}^{*} q \kappa p_{ \pm},
$$

which completes the proof since $q \kappa \geq 0$. The fact that (2) and (3) are equivalent is an easy computation.

Using the above result one can prove an analogue of Proposition 1.5.7 in the charged case.

Proposition 1.5.14. Let $(V, q)$ be a unitary space and let $\mathcal{V}$ be the completion of $V$ w.r.t. the norm induced by $q$. Let $\lambda_{1} \in \mathcal{B}(\mathcal{V}), \lambda_{2} \in \mathcal{B}(\mathcal{V})$ be two-point functions of two pure quasi-free states on $\operatorname{CAR}(V, q)$. Then there exists an operator $r \in \mathcal{U}(\mathcal{V})$ s.t. $\lambda_{2}=r^{*} \lambda_{1} r$, where $r^{*} \in \mathcal{B}(\mathcal{H})$ stands for the Hilbert-space adjoint.

1.5.3. Neutral bosons. Let $(X, \sigma)$ be a symplectic space.

Quasi-free states on $\operatorname{CCR}(X, \sigma)$ could be formally defined as those $\omega$ which satisfy

$$
\omega(\phi(f) \phi(g))=\eta(f, g)+\frac{\mathrm{i}}{2} \sigma(f, g), \quad f, g \in X
$$

for some symmetric $\eta \in L\left(X, X^{\#}\right)$. In the bosonic case, however, field operators $\phi^{\pi}(f)$ are always unbounded and there are no corresponding elements $\phi(f)$ in the $C^{*}$ algebra $\operatorname{CCR}(X, \sigma)$. This problem is avoided in the definition below by considering an exponentiated version of (1.5.8).

Definition 1.5.15. A state $\omega$ on $\operatorname{CCR}(X, \sigma)$ is called a (bosonic, neutral) quasi-free state if there exists a symmetric form $\eta$ on $X$, called the covariance of $\omega$, such that

$$
\omega(W(f))=\mathrm{e}^{-\frac{1}{2} \eta(f, f)}, \quad f \in X .
$$

Proposition 1.5.16. Let $\omega$ be a quasi-free state on $\operatorname{CCR}(X, \sigma)$ and let $(\mathcal{H}, \pi, \Omega)$ be the associated GNS representation. Then the CCR representation

$$
X \ni f \mapsto W^{\pi}(f):=\pi(W(f)) \in \mathcal{B}(\mathcal{H})
$$


is regular and there exists a unique $\Psi \in \mathcal{H}$ s.t.

$$
\omega(W(f))=\left(\Psi \mid W^{\pi}(f) \Psi\right), \quad f \in X .
$$

Moreover, $\Psi \in \mathcal{H}^{\infty, \pi}$ and

$$
\left(\Psi \mid \phi^{\pi}(f) \phi^{\pi}(g) \Psi\right)=\eta(f, g)+\frac{\mathrm{i}}{2} \sigma(f, g), \quad f, g \in X .
$$

The expression $\left(\Psi \mid \phi^{\pi}(f) \phi^{\pi}(g) \Psi\right)$ can be thought of as a rigorous replacement for the ill-defined object $\omega(\phi(f) \phi(g))$. Another way of making the expression $\omega(\phi(f) \phi(g))$ rigorous is to give up on the $C^{*}$-algebraic picture and consider instead a $*$-algebra which contains $\phi(f)$. This alternative strategy is frequently used in QFT on curved spacetime and the *-algebra used in this context is the so-called Borchers-Uhlmann algebra.

DEFINITION 1.5.17. The two-point function of a (bosonic, neutral) quasi-free state is the sesquilinear hermitian form

$$
\lambda:=\eta_{\mathbb{C}}+\frac{\mathrm{i}}{2} \sigma_{\mathbb{C}} \in L\left(\mathbb{C} X, \overline{\mathbb{C} X}^{*}\right)
$$

Similarly to the fermionic case, we introduce a hermitian sesquilinear form called the charge form

$$
q:=\mathrm{i} \sigma_{\mathbb{C}} \in L\left(\mathbb{C} X, \overline{\mathbb{C} X}^{*}\right) .
$$

The following results are well-known (see, e.g., [AS71], [DG13, Chaps. 17,11]).

Proposition 1.5.18. Let $\eta \in L\left(X, X^{\#}\right)$ be a symmetric form. Then the following are equivalent:

(1) $\eta$ is the covariance of a quasi-free state on $\operatorname{CCR}(X, \sigma)$;

(2) $\eta \geq 0$ on $X$ and

$$
\left|\sigma\left(f_{1}, f_{2}\right)\right| \leq 2\left(\eta\left(f_{1}, f_{1}\right)\right)^{\frac{1}{2}}\left(\eta\left(f_{2}, f_{2}\right)\right)^{\frac{1}{2}}, \quad f_{1}, f_{2} \in X
$$

(3) $\lambda \geq 0$ on $\mathbb{C} X$;

(4) $\lambda \geq 0$ and $\lambda \geq q$ on $\mathbb{C} X$.

Corollary 1.5.19. Let $\lambda \in L\left(\mathbb{C} X, \overline{\mathbb{C} X}^{*}\right)$. Then the following are equivalent:

(1) $\lambda$ is the two-point function of a quasi-free state on $\operatorname{CCR}(X, \sigma)$;

(2) $\lambda \geq 0$ and $\lambda \geq q$.

The subclass of pure quasi-free states can be characterized as follows in terms of their covariances $\eta$.

Proposition 1.5.20. Let $\eta \in L\left(X, X^{\#}\right)$ be symmetric. The following are equivalent:

(1) $\eta$ is the covariance of a pure quasi-free state on $\operatorname{CCR}(X, \sigma)$;

(2) there exists $\mathrm{j} \in S p(X, \sigma)$ s.t. $\mathrm{j}^{2}=-1$ and $2 \eta=\sigma \mathrm{j}$.

Proposition 1.5.21. Let $(X, \sigma)$ be a presymplectic space and let $\eta_{1}, \eta_{2}$ be covariances of two pure quasi-free states on $\operatorname{CCR}(X, \sigma)$. Denote $\mathcal{X}_{1}, \mathcal{X}_{2}$ the completions of $X$ w.r.t. respectively $\eta_{1}, \eta_{2}$. Then there exists an operator $r \in \mathcal{B}\left(\mathcal{X}_{2}, \mathcal{X}_{1}\right) \cap S p(X, \sigma)$ s.t. $\eta_{2}=r^{\mathrm{t}} \eta_{1} r$, where $r^{\mathrm{t}} \in \mathcal{B}\left(\mathcal{X}_{1}^{\#}, \mathcal{X}_{2}^{\#}\right)$ stands for the Banach-space adjoint. 
1.5.4. Charged bosons. Let $(V, q)$ be a pre-unitary space.

Clearly, $\left(V_{\mathbb{R}}, \operatorname{Im} q\right)$ is a real symplectic space and the complex structure $\mathrm{j}$ on $V$ satisfies $\mathrm{j} \in S p\left(V_{\mathbb{R}}, \operatorname{Im} q\right)$.

For coherence of notation we will denote the Weyl CCR $C^{*}$-algebra

$$
\operatorname{CCR}(V, q):=\operatorname{CCR}\left(V_{\mathbb{R}}, \operatorname{Im} q\right) .
$$

By definition, (bosonic) charged quasi-free states are quasi-free states on $\operatorname{CCR}(V, q)$ in the sense of the previous subsection. Let $\omega$ be a quasi-free state on $\operatorname{CCR}(V, q)$ and let $(\mathcal{H}, \pi, \Omega)$ be the associated GNS representation. By Proposition 1.5.16, the corresponding 'neutral' CCR representation $v \mapsto W^{\pi}(v)=\omega(W(v))$ is regular and we have well-defined self-adjoint field operators $\phi^{\pi}(v) \in \mathcal{C}(\mathcal{H})$. If now

$$
\psi^{\pi}(v):=\frac{1}{\sqrt{2}}\left(\phi^{\pi}(v)+\mathrm{i} \phi^{\pi}(\mathrm{j} v)\right), \quad v \in V
$$

then the map

$$
V \ni v \mapsto \psi^{\pi}(v) \in \mathcal{C}(\mathcal{H})
$$

is a charged CCR representation.

In the charged case, physical states are required to satisfy a gauge-invariance condition.

Definition 1.5.22. A state $\omega$ on $\operatorname{CCR}(V, q)$ is called gauge-invariant if

$$
\omega(W(v))=\omega\left(W\left(\mathrm{e}^{\mathrm{j} \theta} v\right)\right), \quad 0 \leq \theta<2 \pi, \quad v \in V .
$$

If $\omega$ is a charged quasi-free state with covariance $\eta$ then gauge-invariance is equivalent to

$$
\mathrm{j} \in O\left(V_{\mathbb{R}}, \eta\right) .
$$

If $\omega$ is a charged quasi-free state on $\operatorname{CCR}(V, q)$, by Proposition 1.5.16 there exists a vector $\Psi \in \mathcal{H}^{\infty, \pi}$ s.t.

$$
\omega(W(v))=\left(\Psi \mid W^{\pi}(v) \Psi\right), \quad v \in V .
$$

If $\omega$ is additionally gauge-invariant then one can show that

$$
\left(\Psi \mid \psi^{\pi}(v) \psi^{\pi}(w) \Psi\right)=\left(\Psi \mid \psi^{\pi *}(v) \psi^{\pi *}(w) \Psi\right)=0, \quad v, w \in V
$$

DEFINITION 1.5.23. The two-point function of a (bosonic, charged) gauge-invariant quasi-free state $\omega$ is the hermitian form $\lambda \in L\left(V, \bar{V}^{*}\right)$ given by

$$
\lambda(v, w):=\left(\Psi \mid \psi^{\pi}(v) \psi^{\pi *}(w) \Psi\right), \quad v, w \in V .
$$

The charge density of $\omega$ is the hermitian form

$$
c(v, w):=\left(\Psi \mid \psi^{\pi *}(w) \psi^{\pi}(v) \Psi\right), \quad v, w \in V .
$$

The following propositions are the analogues of Propositions 1.5.4, 1.5.20 and 1.5.21. We sketch their proofs for the reader's convenience.

Proposition 1.5.24. Let $\lambda \in L\left(V, \bar{V}^{*}\right)$. Then the following are equivalent:

(1) $\lambda$ is the two-point function of a gauge-invariant quasi-free state on $\operatorname{CCR}(V, q)$; 
(2) $\lambda \geq 0$ and $\lambda \geq q$.

Proof. In terms of the self-adjoint fields $\phi(v)$, using the CCRs we compute

$$
\omega(\phi(v) \phi(w))=\operatorname{Re}\left(\lambda-\frac{1}{2} q\right)\left(v_{1}, v_{2}\right)+\frac{\mathrm{i}}{2} \operatorname{Im} q\left(v_{1}, v_{2}\right),
$$

hence $\eta=\operatorname{Re}\left(\lambda-\frac{1}{2} q\right)$. Since $\omega$ is gauge-invariant,

$$
\mathrm{j} \in O\left(V_{\mathbb{R}}, \eta\right) \cap S p\left(V_{\mathbb{R}}, \operatorname{Im} q\right)=O\left(V_{\mathbb{R}}, \eta\right) \cap O\left(V_{\mathbb{R}}, \operatorname{Re} q\right) .
$$

From this fact we deduce that $\eta \geq 0 \Leftrightarrow \lambda \geq \frac{1}{2} q$, and that the second condition in Proposition 1.5.4 (with $\sigma$ replaced by $\operatorname{Im} q$ ) is equivalent to

$$
\pm q \leq 2 \lambda-q .
$$

These three conditions are equivalent to $\lambda \geq 0, \lambda \geq q$.

Proposition 1.5.25. Let $\lambda \in L\left(V, \bar{V}^{*}\right)$ be hermitian. Then the following are equivalent:

(1) $\lambda$ is the two-point function of a pure gauge-invariant quasi-free state on $\mathrm{CCR}(V, q)$;

(2) there exists an involution $\kappa \in U(V, q)$ s.t. $q \kappa \geq 0$ and $\lambda=\frac{1}{2} q(\mathbf{1}+\kappa)$;

(3) $\lambda \geq \frac{1}{2} q$ and $\lambda q^{-1} \lambda=\lambda$.

Proof. By Proposition 1.5.20 the state $\omega$ is pure iff there exists an anti-involution $\mathrm{j} \in S p\left(V_{\mathbb{R}}, \operatorname{Im} q\right)$ such that

$$
2 \eta=(\operatorname{Im} q) \mathrm{j} .
$$

Since $\mathrm{j} \in O\left(V_{\mathbb{R}}, \eta\right) \cap S p\left(V_{\mathbb{R}}, \operatorname{Im} q\right)$ we obtain that $\mathrm{j} \in U(V, q)$, i.e. $\mathrm{j}$ is $\mathbb{C}$-linear and pseudo-unitary for $q$. From (1.5.16) we then get that $2 \lambda-q=\sigma \mathrm{j}$. Setting $\kappa=-\mathrm{ij}$ we see that $\kappa \in U(V, q)$ and $\lambda=\frac{1}{2} q(\mathbf{1}+\kappa)$. Therefore (1) is equivalent to

$$
\text { (4) } \lambda \geq 0, \lambda \geq q, \lambda=\frac{1}{2} q(\mathbf{1}+\kappa), \kappa^{2}=\mathbf{1}, \kappa \in U(V, q) \text {. }
$$

(4) clearly implies (2). Let us prove the converse implication. Set $p_{ \pm}:=\frac{1}{2}(\mathbf{1} \pm \kappa)$. Clearly $p_{ \pm}$are projections with $p_{ \pm}^{*} q=q p_{ \pm}, \kappa p_{ \pm}= \pm p_{ \pm}$, and

$$
\lambda \geq 0, \lambda \geq q \Leftrightarrow \pm q p_{ \pm} \geq 0 .
$$

Now we have

$$
q p_{ \pm}=q p_{ \pm}^{2}=p_{ \pm}^{*} q p_{ \pm}= \pm p_{ \pm}^{*} q \kappa p_{ \pm},
$$

which completes the proof since $q \kappa \geq 0$. The fact that (2) and (3) are equivalent is an easy computation.

Proposition 1.5.26. Let $\lambda_{1}, \lambda_{2}$ be two-point functions of two pure, gauge-invariant quasi-free states on $\operatorname{CCR}(V, q)$. Then there exists $r \in U(V, q)$ s.t. $\lambda_{2}=r^{*} \lambda_{1} r$.

Proof. We introduce the real covariances $\eta_{1}, \eta_{2}$. By Proposition 1.5.7 there exists $r \in S p\left(V_{\mathbb{R}}, \operatorname{Im} q\right)$ with $\eta_{2}=r^{\mathrm{t}} \eta_{1} r$. Using the gauge-invariance of the two states we obtain that $r \mathrm{j}=\mathrm{j} r$, hence $r \in U(V, q)$. 
1.5.5. Complexification of quasi-free states. The characterization of twopoint functions of quasi-free states we discussed in this chapter yields the following relation between the neutral and charge case.

Theorem 1.5.27. Let $(X, \nu)$ be an orthogonal space and let $\omega$ be a quasi-free state on $\operatorname{CAR}(X, \nu)$ with two-point function $\lambda$. Set

$$
\tilde{V}:=\mathbb{C} X, \quad \tilde{q}:=2 \nu_{\mathbb{C}} .
$$

Then there exists a unique gauge-invariant quasi-free state $\tilde{\omega}$ on $\operatorname{CAR}(\tilde{V}, \tilde{q})$ with two-point function $\tilde{\lambda}=\lambda$. Moreover, $\tilde{\omega}$ is a pure state iff $\omega$ is pure.

Conversely, let $(V, q)$ be a pre-unitary space and let $\omega$ be a gauge-invariant quasifree state on $\operatorname{CAR}(V, q)$ with two-point function $\lambda$. Set

$$
\tilde{X}:=V_{\mathbb{R}}, \quad \tilde{\nu}:=\frac{1}{2} \operatorname{Re} q .
$$

Then there exists a unique quasi-free state $\tilde{\omega}$ on $\operatorname{CAR}(\tilde{X}, \tilde{\nu})$ with two-point function $\tilde{\lambda}=\lambda$. Moreover, $\tilde{\omega}$ is a pure state iff $\omega$ is pure.

The bosonic analogue reads:

TheOrem 1.5.28. Let $(X, \sigma)$ be a symplectic space and let $\omega$ be a quasi-free state on $\operatorname{CCR}(X, \sigma)$ with two-point function $\lambda$. Set

$$
\tilde{V}:=\mathbb{C} X, \quad \tilde{q}:=\mathrm{i} \sigma_{\mathbb{C}} .
$$

Then there exists a unique gauge-invariant quasi-free state $\tilde{\omega}$ on $\operatorname{CCR}(\tilde{V}, \tilde{q})$ with two-point function $\tilde{\lambda}=\lambda$. Moreover, $\tilde{\omega}$ is a pure state iff $\omega$ is pure.

Conversely, let $(V, q)$ be a pre-unitary space and let $\omega$ be a gauge-invariant quasifree state on $\operatorname{CCR}(V, q)$ with two-point function $\lambda$. Set

$$
\tilde{X}:=V_{\mathbb{R}}, \quad \tilde{\sigma}:=\operatorname{Im} q .
$$

Then there exists a unique quasi-free state $\tilde{\omega}$ on $\operatorname{CCR}(\tilde{X}, \tilde{\sigma})$ with two-point function $\tilde{\lambda}=\lambda$. Moreover, $\tilde{\omega}$ is a pure state iff $\omega$ is pure.

It follows that, possibly after complexifying the real orthogonal or symplectic space, one can always restrict the discussion to gauge-invariant quasi-free states on a pre-unitary space $(V, q)$ (where $q$ is required to be positive in the fermionic case).

In the sequel we will henceforth only consider gauge-invariant quasi-free states, and often call them simply quasi-free states.

1.5.6. Charge conjugation. If the pre-unitary space $(V, q)$ possesses some discrete symmetries, it is natural to consider classes of states which preserve these symmetries. A common example of a discrete symmetry is charge conjugation.

Definition 1.5.29. Let $(V, q)$ be a pre-unitary space. An anti-linear map $\kappa: V \rightarrow V$ is said to be a charge conjugation if $\kappa^{2}=\mathbf{1}$ or $\kappa^{2}=-\mathbf{1}$ and

$$
q(\kappa v, \kappa w)=q(w, v), \quad v, w \in V
$$


in the fermionic case, respectively

$$
q(\kappa v, \kappa w)=-q(w, v), \quad v, w \in V .
$$

in the bosonic case.

One says that a quasi-free state $\omega$ on $\operatorname{CAR}(V, q)$ or $\operatorname{CCR}(V, q)$ is invariant under charge conjugation if

$$
\omega\left(\psi^{*}(v) \psi(w)\right)=\omega\left(\psi(\kappa v) \psi^{*}(\kappa w)\right), \quad v, w \in V .
$$

Strictly speaking, in the bosonic case, the formal expression $\omega\left(\psi^{*}(v) \psi(w)\right)$ has to be understood as in Definition 1.5.23.

If there exists a charge conjugation on $(V, q)$ then given a quasi-free state $\omega$ on $\operatorname{CAR}(V, q)$ or $\operatorname{CCR}(V, q)$, invariance under charge conjugation means that the twopoint function $\lambda$ and charge density $c$ are related by

$$
c(v, w)=\lambda(\kappa w, \kappa v), \quad v, w \in V .
$$

Therefore, a hermitian sesquilinear form $\lambda$ on $V$ is the two-point function of a quasifree state which is invariant under charge conjugation iff

$$
\lambda(v, v) \geq 0, \quad \lambda(v, w)+\lambda(\kappa w, \kappa v)=q(v, w) \quad v, w \in V
$$

in the fermionic case, and

$$
\lambda(v, v) \geq 0, \quad \lambda(v, w)-\lambda(\kappa w, \kappa v)=q(v, w) \quad v, w \in V
$$

in the bosonic case. 



\section{CHAPTER 2}

\section{Normally and pre-normally hyperbolic operators}

In non-interacting Quantum Field Theory, the classical space which is quantized is the space of smooth solutions of the free equations of motions: the Klein-Gordon equation in the case of spin-0 fields, and the Dirac equation in the case of spin- $\frac{1}{2}$ fields. This construction can be carried through on a curved spacetime provided that the equation of motion has a reasonable global theory, as is the case on globally hyperbolic spacetimes. On the same lines, it is possible to consider a coupling to a smooth, real valued external electromagnetic potential, which may possibly vary in time.

In this chapter we collect basic results needed for the construction of quantum fields on globally hyperbolic spacetimes in the spin- 0 and spin- $-\frac{1}{2}$ case. Our discussion follows the references [BGP08, Dim82, BG11], although our results are more general in the respect that we allow for smooth external potentials in formulating the Cauchy problem. We also discuss an alternative approach to the Klein-Gordon equation, usually written in the form

$$
\left[-\left(\nabla_{\mu}+\mathrm{i} A_{\mu}\right)\left(\nabla^{\mu}+\mathrm{i} A^{\mu}\right)+m^{2}\right] f=0 .
$$

Namely, we observe that this is equivalent to the system

$$
\left(\begin{array}{cc}
\mathrm{i}^{-1} n^{\mu}\left(\nabla_{\mu}+\mathrm{i} A_{\mu}\right) & 1 \\
-h^{\mu \nu}\left(\nabla_{\mu}+\mathrm{i} A_{\mu}\right)\left(\nabla_{\nu}+\mathrm{i} A_{\nu}\right)+m^{2} & \mathrm{i}^{-1} n^{\mu}\left(\nabla_{\mu}+\mathrm{i} A_{\mu}\right)
\end{array}\right)\left(\begin{array}{l}
f_{0} \\
f_{1}
\end{array}\right)=0
$$

by substituting $f_{0}=f$ and $f_{1}=\mathrm{i}^{-1} n^{\mu}\left(\nabla_{\mu}+\mathrm{i} A_{\mu}\right)$, where $n^{\mu}$ and $h^{\mu \nu}$ are constructed once a so-called Gaussian foliation of $(M, g)$ by Cauchy surfaces is given. The twocomponent Klein-Gordon equation (2.0.2) is a generalization of the equation considered by Feshbach and Villars in the flat case already in the 1950's [FV58]. It has the advantage of having many properties in common with the Dirac equation and it also proves to be a helpful auxiliary device in deriving formulae for the solution of the Cauchy problem associated to (2.0.1). In the static case, $n^{\mu} \nabla_{\mu}$ is interpreted as the derivative in the time direction - this fact will be used in Chapter 5, where (2.0.2) will be solved using a one-parameter group of non-selfadjoint operators.

The well-known results on the Klein-Gordon equation that we will recall in this chapter (with external potentials or without) rely strongly on the fact that it is normally hyperbolic, i.e., the highest derivative term equals $-g^{\mu \nu} \partial_{\mu} \partial_{\nu}$. The analysis of the Dirac equation and two-component Klein-Gordon equation leads us to consider the more general class of pre-normally hyperbolic differential operators $D$, which have the property that $D \widetilde{D}$ is normally hyperbolic for some operator $\widetilde{D}$. This allows to deduce properties like existence of advanced and retarded propagators for $D$ from analogous facts in the normally hyperbolic case. Pre-normally hyperbolic of order 
one were studied in [Müh07, Müh11], the original motivation coming from the study of fermionic fields with spin higher or equal $\frac{1}{2}$. We extend the results of Mühlhoff on well-posedness of the Cauchy problem to a more general class of pre-normally hyperbolic operators, which includes in particular (2.0.2).

Next, we outline the well-known construction of the pre-unitary space which describes the classical field theory associated to a pre-normally hyperbolic $D$ on a hermitian bundle $\mathscr{V}$. We show that the pre-unitary spaces which correspond to the oneand two-component form of the Klein-Gordon equation are isomorphic, thus the two quantization problems are equivalent. Finally, we briefly discuss under what condition does a pair of distributions $\Lambda^{( \pm)}$on $M \times M$ define a two-point function of a quasi-free states on the respective CAR or CCR algebra, in the generality appropriate for systems with no charge conjugation.

The first few sections contain a brief introduction to globally hyperbolic spacetimes and differential operators and make use of expository references such as [BGP08, Pfä09].

\subsection{Globally hyperbolic spacetimes}

Let us denote by $\mathbb{R}^{1, d}$ the $n=1+d$ Minkowski space, which is simply $\mathbb{R}^{n}$ equipped with the symmetric bilinear form

$$
x \cdot y=\sum_{\mu, \nu} \eta_{\mu \nu} x_{\mu} y_{\nu}, \quad x, y \in \mathbb{R}^{n},
$$

where $\mu, \nu=0,1, \ldots, d$ and we adopt the convention

$$
\eta_{\mu \nu}= \begin{cases}-1, & \mu=\nu=0 \\ 1, & \mu=\nu, \quad \mu \neq 0 \\ 0, & \mu \neq \nu\end{cases}
$$

More generally, we call a non-degenerate, bilinear symmetric form $\eta$ a Lorentzian product on an $n$-dimensional vector space $V$ if there exists a basis $\left\{e_{\mu}\right\}_{\mu=0, \ldots, n}$ of $V$ such that

$$
\eta\left(e_{\mu}, e_{\nu}\right)=\eta_{\mu \nu} .
$$

We write in short $x^{2}=\eta(x, x)$. A vector $x \in V$ is called time-like if $x^{2}<0$, light-like if $x^{2}=0$ and $x \neq 0$, null if $x^{2}=0$ and space-like if $x^{2}>0$ or $x=0$.

For $n \geq 2$ the set of timelike vectors has two connected components. Fixing a timeorientation means that one chooses one of those two components and call its elements future-directed. Elements of the other component are then called past-directed.

Let $(M, g)$ be a Lorentzian manifold ${ }^{1}$ of dimension $n \geq 2$ and signature $(-,+, \ldots,+)$. This means that $M$ is a smooth manifold and $g$ is a Lorentzian metric, i.e., to each point $x \in M$ it associates in a smooth way a Lorentzian scalar product $g_{x}$ on the tangent space $T_{x} M$. More precisely, if $x=\left(x_{\mu}\right)_{\mu=0, \ldots, d}: U \rightarrow \tilde{U} \subset \mathbb{R}^{n}$ are local coordinate maps and $\partial_{\mu}$ are the corresponding coordinate vector fields, then

\footnotetext{
${ }^{1}$ More precisely, we always consider Hausdorff, paracompact, connected smooth manifolds.
} 
the functions

$$
g_{\mu \nu}:=g\left(\partial_{\mu}, \partial_{\nu}\right): \tilde{U} \rightarrow \mathbb{R}
$$

are required to be smooth.

We would like to assign to each $x \in M$ a time-orientation on $T_{x} M$ in a continuous way. This corresponds to choosing a continuous time-like vector field $\tau$; its value at $x$ then determines a time-orientation. Such vector field $\tau$ does however not always exist on a generic Lorentzian manifold.

Definition 2.1.1. A Lorentzian manifold $(M, g)$ is said to be time-orientable if there exists a continuous timelike vector field $\tau$ on $M$.

From now on we will consider only connected, time-oriented Lorentzian manifolds (i.e., time-orientable ones equipped with a continuous timelike vector field) and call them spacetimes.

A curve $\gamma: \mathcal{I} \rightarrow M$ is said to be causal if the tangent vectors along $\gamma$ are timelike or light-like. Additionally, one says that it is extendible if there exists another causal curve $\tilde{\gamma}: \tilde{\mathcal{I}} \rightarrow M$ and a subinterval $\mathcal{J} \subset \mathcal{I}$ together with a reparametrisation $\phi: \mathcal{J} \rightarrow \mathcal{I}$, such that $\gamma \circ \phi=\left.\tilde{\gamma}\right|_{\mathcal{J}}$

Definition 2.1.2. A hypersurface $\Sigma$ of $M$ is called a Cauchy surface if each inextendible timelike curve in $M$ intersects $\Sigma$ at exactly one point. A spacetime $(M, g)$ is called globally hyperbolic if there exists a Cauchy surface in $M$.

The existence of a Cauchy surface implies that the metric has a quite specific, diagonal form, described in the theorem below. The fact that one can choose $\Sigma$ to be smooth is a relatively recent result due to Bernal and Sánchez [BS05].

TheOREM 2.1.3. A spacetime is globally hyperbolic iff it is isometric to $\mathbb{R} \times \Sigma$ with metric $-\vartheta d t^{2}+g_{t}$, where $\vartheta>0$ is a smooth function, $g_{t}$ is a Riemannian metric on $\Sigma$ depending smoothly on $t \in \mathbb{R}$ and each $\{t\} \times \Sigma$ is a smooth spacelike Cauchy surface in $M$.

In what follows we will consider only smooth Cauchy surfaces.

The geometrical concept which is essential in the construction of a Quantum Field Theory is the causal structure of the underlying spacetime. The causal future $J_{+}(x)$ of a point $x \in M$ (resp. the causal past) $J_{-}(x)$ ), is defined to be the set of all $y \in M$ that can be reached from $x$ by a causal future-directed (resp. past-directed) curve. By convention, $x \in J_{+}(x)$ and $x \in J_{-}(x)$. The causal future and causal past of a subset $U \subset M$ are by definition the sets

$$
J_{ \pm}(U):=\bigcup_{x \in U} J_{ \pm}(x) .
$$

We will need later on the following important fact, discussed in detail for instance in [BGP08].

THEOREM 2.1.4. If $(M, g)$ is globally hyperbolic and $K, K^{\prime} \subset M$ are compact sets, then $J_{-}(K) \cap J_{+}\left(K^{\prime}\right)$ is compact. 
An essential feature of globally hyperbolic spacetimes is that they possess no timelike causal curves nor null ones. Furthermore, there is a difficult result which states that if a spacetime $(M, g)$ has this property and the assertion of Theorem 2.1.4 holds true, then it must be globally hyperbolic [BS07].

\subsection{Distributions on manifolds}

Let $(M, g)$ be a pseudo-Riemannian manifold and let $\mathscr{V}$ be a finite rank vector bundle with base space $M$. We will denote $\mathscr{V}^{*}$ the dual bundle of $\mathscr{V}$ and $\overline{\mathscr{V}}^{*}$ the antidual bundle, i.e., the bundle whose fiber at $x \in M$ consists of anti-linear functionals on $\mathscr{V}_{x}$. If $\mathscr{W}$ is another finite rank vector bundle over $M$, the endomorphism bundle will be denoted $\mathscr{L}(\mathscr{V}, \mathscr{W})$.

We denote $\mathscr{E}(M, \mathscr{V})$ the space of smooth sections of $\mathscr{V}$, and $\mathscr{D}(M, \mathscr{V})$ the space of compactly supported ones, endowed with their standard Fréchet space topology.

Assume now $M$ is orientable. In that case we have a uniquely defined volume form $\Omega_{g}$. In local coordinates,

$$
\Omega_{g}=|g|^{\frac{1}{2}} d x^{0} \wedge \cdots \wedge d x^{n-1}, \quad|g|:=\operatorname{det}\left[g_{\mu \nu}\right] .
$$

The associated volume density will be denoted $\mu_{g}$. In local coordinates, $\mu_{g}=$ $|g|^{\frac{1}{2}} d x^{0} \cdots d x^{n-1}$.

The space of $\mathscr{V}^{*}$-valued distributions, denoted $\mathscr{D}^{\prime}\left(M, \mathscr{V}^{*}\right)$, is by definition the dual space of $\mathscr{D}(M, \mathscr{V})$.

Using the volume form $\Omega_{g}$ we can identify $\mathscr{E}\left(M, \mathscr{V}^{*}\right)$ with a subspace of $\mathscr{D}^{\prime}\left(M, \mathscr{V}^{*}\right)$. Indeed, to each $u \in \mathscr{E}\left(M, \mathscr{V}^{*}\right)$ we can associate a linear functional on $\mathscr{D}(M, \mathscr{V})$ by

$$
\langle u, f\rangle:=\int_{M}\langle u, f\rangle_{x} d \Omega_{g}, \quad f \in \mathscr{D}(M, \mathscr{V}),
$$

where $\langle\cdot, \cdot\rangle_{x}$ stands for fiberwise evaluation of elements of $\mathscr{V}_{x}^{*}$ on $\mathscr{V}_{x}$.

\subsection{Operators on manifolds}

Let us now turn our attention to operators on pseudo-Riemannian manifolds.

Let $\mathscr{V}, \mathscr{W}$ be finite rank vector bundles over $M$ and let $A: \mathscr{D}(M, \mathscr{V}) \rightarrow \mathscr{D}^{\prime}(M, \mathscr{W})$ be a continuous operator. The Schwartz kernel theorem states that there is a unique distribution $K_{A} \in \mathscr{D}^{\prime}\left(M \times M, \mathscr{V} \otimes \mathscr{W}^{*}\right)$ (which we call the Schwartz kernel of $A$ ) such that

$$
\langle A f, g\rangle=\left\langle K_{A}, g \otimes f\right\rangle, \quad f \in \mathscr{D}(M, \mathscr{V}), \quad g \in \mathscr{D}\left(M, \mathscr{W}^{*}\right) .
$$

Conversely, given $K_{A} \in \mathscr{D}^{\prime}\left(M \times M, \mathscr{V} \otimes \mathscr{W}^{*}\right)$, the above equation defines a continuous operator $A: \mathscr{D}(M, \mathscr{V}) \rightarrow \mathscr{D}^{\prime}(M, \mathscr{W})$.

The transpose operator of $A$ is the operator $A^{\mathrm{t}}: \mathscr{D}\left(M, \mathscr{W}^{*}\right) \rightarrow \mathscr{D}^{\prime}\left(M, \mathscr{V}^{*}\right)$ with Schwartz kernel

$$
K_{A^{\mathrm{t}}}:=\operatorname{Exch} K_{A} \in \mathscr{D}^{\prime}\left(M \times M, \mathscr{W}^{*} \otimes \mathscr{V}\right)
$$

where the operation Exch exchanges the two arguments of $K_{A}$, i.e.

$$
\langle\operatorname{Exch} K, f \otimes g\rangle=\langle K, g \otimes f\rangle, \quad f \in \mathscr{D}(M, \mathscr{V}), \quad g \in \mathscr{D}\left(M, \mathscr{W}^{*}\right)
$$


under the identification $\mathscr{L}\left(\mathscr{W}^{*}, \mathscr{V}^{*}\right) \cong \mathscr{L}(\mathscr{V}, \mathscr{W})$ (or in a more formal notation, $\left.\operatorname{Exch} K(x, y)=K^{\mathrm{t}}(y, x)\right)$. The transpose operator satisfies

$$
\langle g, A f\rangle=\left\langle A^{\mathrm{t}} g, f\right\rangle, \quad f \in \mathscr{D}(M, \mathscr{V}), \quad g \in \mathscr{D}\left(M, \mathscr{W}^{*}\right) .
$$

This relation can be slightly generalized. Indeed,

$$
\int\langle g, A f\rangle_{x} d \Omega_{g}=\int\left\langle A^{\mathrm{t}} g, f\right\rangle_{x} d \Omega_{g}
$$

holds for any $f \in \mathscr{E}(M, \mathscr{V}), g \in \mathscr{E}\left(M, \mathscr{W}^{*}\right)$ such that $\operatorname{supp} f \cap \operatorname{supp} g$ is compact. We will use frequently this fact in the next sections.

The formal dual operator of $A$ is the operator $A^{*}: \mathscr{D}\left(M, \overline{\mathscr{W}}^{*}\right) \rightarrow \mathscr{D}^{\prime}\left(M, \overline{\mathscr{V}}^{*}\right)$ with Schwartz kernel

$$
K_{A^{*}}:=\operatorname{Exch} \overline{K_{A}} \in \mathscr{D}^{\prime}\left(M \times M, \overline{\mathscr{W}}^{*} \otimes \overline{\mathscr{V}}\right)
$$

where the bar stands for ordinary complex conjugation. The formal dual satisfies

$$
\langle\bar{g}, A f\rangle=\left\langle\overline{A^{*} g}, f\right\rangle, \quad f \in \mathscr{D}(M, \mathscr{V}), \quad g \in \mathscr{D}\left(M, \overline{\mathscr{W}}^{*}\right) .
$$

Suppose now that $A^{\text {t }}$ maps $\mathscr{D}(M, \mathscr{V})$ continuously to $\mathscr{E}(M, \mathscr{V})$. In such case one says that $A$ is regular. By duality we can extend $A$ to an operator acting on compactly supported distributions, namely

$$
\begin{aligned}
& A: \mathscr{E}^{\prime}(M, \mathscr{V}) \rightarrow \mathscr{D}^{\prime}(M, \mathscr{V}), \\
&\langle A u, f\rangle:=\left\langle u, A^{\mathrm{t}} f\right\rangle, \quad u \in \mathscr{E}^{\prime}(M, \mathscr{V}), \quad f \in \mathscr{D}\left(M, \mathscr{W}^{*}\right) .
\end{aligned}
$$

It is possible to extend the domain of definition of $A$ in a different way if it is a properly supported operator, i.e., if both projections $\pi_{1}, \pi_{2}: M \times M \rightarrow M$ are proper when restricted to supp $K_{A}\left(K_{A}\right.$ being the Schwartz kernel of $\left.A\right)$. This property entails that $A$ maps $\mathscr{D}$ to $\mathscr{E}$ and therefore we can extend it by duality to act $\mathscr{E} \rightarrow \mathscr{D}^{\prime}$. Operators which are both regular and properly supported are of special interest because their composition is always well-defined.

2.3.1. Differential operators. A differential operator of order $m$ is a linear map $\mathscr{E}(M, \mathscr{V}) \rightarrow \mathscr{E}(M, \mathscr{V})$ which in local coordinates $x_{\mu}$ has the form

$$
P=\sum_{|\alpha| \leq m} a_{\alpha}(x) \partial_{x}^{\alpha},
$$

where $\alpha=\left(\alpha_{0}, \ldots \alpha_{d}\right) \in \mathbb{N}_{0}^{n}$ are multi-indices, $|\alpha|=\alpha_{0}+\cdots+\alpha_{d}, \partial_{x}^{\alpha}:=\partial_{0}^{\alpha_{0}} \ldots \partial_{d}^{\alpha_{d}}$ and $a_{\alpha} \in \mathscr{E}(M, \mathscr{L}(\mathscr{V}))$.

The principal symbol $\sigma_{P}$ of the differential operator (2.3.3) is the linear map $\sigma_{P}: T^{*} M \rightarrow \mathscr{L}(\mathscr{V})$ given locally by

$$
\sigma_{P}(x, \xi)=\sum_{|\alpha|=m} a_{\alpha}(x) \xi^{\alpha}, \quad(x, \xi) \in T^{*} M,
$$

where $\xi^{\alpha}=\xi_{0}^{\alpha_{0}} \cdots \xi_{d}^{\alpha_{d}}$ and $\xi=\xi_{\mu} d x^{\mu}$. We will often abbreviate $\sigma_{P}(x, \xi) \equiv \sigma_{P}(\xi)$. The symbol of order $l$, denoted $\sigma_{P}^{l}$, is obtained by replacing the sum over $|\alpha|=m$ in (2.3.4) by a sum over $|\alpha|=l$.

Differential operators act locally, i.e. $\operatorname{supp} P u \subset \operatorname{supp} u$ for all $u \in \mathscr{D}^{\prime}(M, \mathscr{V})$. As 
a consequence, the support of the Schwartz kernel of $P$ is contained in the diagonal $\Delta_{M} \subset M \times M$ and thus $P$ is properly supported. Moreover, $P$ maps continuously $\mathscr{D} \rightarrow \mathscr{D}$ (in particular, it is regular) and we can extend it by duality to an operator $\mathscr{D}^{\prime} \rightarrow \mathscr{D}^{\prime}$. The transpose $P^{\mathrm{t}}$ is a differential operator and in local coordinates it takes the form

$$
P^{\mathrm{t}}=\sum_{|\alpha| \leq m} a_{\alpha}^{\mathrm{t}}(x)(-1)^{|\alpha|} \partial_{x}^{\alpha} .
$$

\subsection{Normally and pre-normally hyperbolic operators}

Let $(M, g)$ be a pseudo-Riemannian manifold and let $\mathscr{V}$ be a smooth, finite rank complex vector bundle on a spacetime $M$. We denote by $\nabla$ the Levi-Civita connection.

Let $A=A_{\mu} d x^{\mu} \in \Omega^{1}(M, \mathscr{L}(\mathscr{V}))$ be a real-valued smooth one-form and let $m \in \mathscr{E}(M, \mathscr{L}(\mathscr{V}))$ be a real-valued smooth section. The differential form $A$ has the interpretation of a background electromagnetic potential and $m$ is a squared mass term which is for the moment allowed to vary in space and time. The minimally coupled Klein-Gordon operator in external electromagnetic potential $A$ is the differential expression

$$
\begin{aligned}
P & =-\left(\nabla_{\mu}+\mathrm{i} A_{\mu}\right)\left(\nabla^{\mu}+\mathrm{i} A^{\mu}\right)+m^{2} \\
& =-|g|^{-\frac{1}{2}}\left(\partial_{\mu}+\mathrm{i} A_{\mu}\right)|g|^{\frac{1}{2}} g^{\mu \nu}\left(\partial_{\nu}+\mathrm{i} A_{\nu}\right)+m^{2}
\end{aligned}
$$

where

$$
|g|=\operatorname{det}\left[g_{\mu \nu}\right], \quad\left[g^{\mu \nu}\right]:=\left[g_{\mu \nu}\right]^{-1} .
$$

The operator (2.4.1) is normally hyperbolic in the following sense.

Definition 2.4.1. A differential operator $P: \mathscr{E}(M, \mathscr{V}) \rightarrow \mathscr{E}(M, \mathscr{V})$ is said to be normally hyperbolic if in local coordinates it takes the form

$$
P=-g^{\mu \nu} \partial_{\mu} \partial_{\nu}+R,
$$

where $R$ is a differential operator of order less or equal 1.

Equivalently, this means that the principal symbol $\sigma_{P}(x, \xi)$ of $P$ is equal to

$$
\sigma_{P}(x, \xi)=g^{-1}(\xi, \xi) \operatorname{id}_{\mathscr{V}_{x}}, \quad(x, \xi) \in T^{*} M
$$

where the symbol $g^{-1}$ is used to denote the inverse metric on $T^{*} M$. Normally hyperbolic operators on globally hyperbolic spacetimes are a well-studied topic and an exhaustive discussion of the well-posedness of the corresponding Cauchy problem, local and global existence of solutions, etc., can be found in the monographs [Fri75, BGP08, Wal12b].

To include for instance the Dirac operator in our discussion we need a more general definition.

Definition 2.4.2. We say that a differential operator $D: \mathscr{E}(M, \mathscr{V}) \rightarrow \mathscr{E}(M, \mathscr{V})$ is pre-normally hyperbolic if there exists a differential operator $\widetilde{D}$ such that $D \widetilde{D}$ is normally hyperbolic. 
A short investigation of the principal symbols shows that if $D, \widetilde{D}$ are differential operators such that $D \widetilde{D}$ is normally hyperbolic then also $\widetilde{D} D$ is normally hyperbolic. According to the above definition, a differential operator of order larger than 1 is allowed to be pre-normally hyperbolic and in particular each normally hyperbolic operator is pre-normally hyperbolic. This differs from the convention adopted for instance in [Müh11], but will allow us to consider at once the Klein-Gordon equation in its two-component form.

2.4.1. Retarded and advanced propagators. The key property needed in the construction of the non-interacting fields is the existence of retarded and advanced propagators.

Definition 2.4.3. Let $D: \mathscr{E}(M, \mathscr{V}) \rightarrow \mathscr{E}(M, \mathscr{V})$ be a differential operator. We call linear maps $S_{ \pm} \in \mathscr{D}(M, \mathscr{V}) \rightarrow \mathscr{E}(M, \mathscr{V})$ advanced/ resp. retarded propagators for $D$ if they satisfy

$$
\begin{aligned}
& \text { (1) } D S_{ \pm} f=f \\
& \text { (2) } S_{ \pm} D f=f \\
& \text { (3) } \operatorname{supp}\left(S_{ \pm} f\right) \subset J_{ \pm}(\operatorname{supp} f), \quad f \in \mathscr{D}(M, \mathscr{V}) .
\end{aligned}
$$

If $S_{-}$(resp. $S_{+}$) is continuous as a map $\mathscr{D} \rightarrow \mathscr{D}^{\prime}$, we can associate a distribution by Schwartz' kernel theorem; it is usually called the retarded fundamental solution (resp. advanced fundamental solution). In the literature, retarded (advanced) propagators are also called alternatively retarded (advanced) Green's operators and retarded (advanced) fundamental solutions are then called retarded (advanced) Green's functions.

The proof of the fundamental result below can be found for instance in [BGP08].

THEOREM 2.4.4. In a globally hyperbolic spacetime, any normally hyperbolic operator $P$ admits unique advanced and retarded propagators $E_{+}, E_{-}$.

We deduce an analogous result for pre-normally hyperbolic operators using a generalization of the method proposed initially by Dimock in the context of the Dirac operator [Dim82]. We follow closely the work of Mühlhoff [Müh11], except that we do not assume that $D$ is an operator of order one.

LEMma 2.4.5. Let $P: \mathscr{E}(M, \mathscr{V}) \rightarrow \mathscr{E}(M, \mathscr{V})$ be a differential operator and assume it admits advanced/retarded propagators $E_{ \pm}$. Then $P^{\mathrm{t}}$ has a unique advanced propagator $E_{-}^{\mathrm{t}}$ (the transpose of $E_{-}$) and a unique retarded one $E_{+}^{\mathrm{t}}$ (the transpose of $\left.E_{+}\right)$.

Proof. For any $u \in \mathscr{E}\left(M, \mathscr{V}^{*}\right), f \in \mathscr{E}(M, \mathscr{V})$ with supp $u \cap \operatorname{supp} f$ compact, we have

$$
\begin{aligned}
\int_{M}\left\langle E_{ \pm}^{\mathrm{t}} u, f\right\rangle_{x} d \Omega_{g} & =\int_{M}\left\langle E_{ \pm}^{\mathrm{t}} u, P E_{\mp} f\right\rangle_{x} d \Omega_{g} \\
& =\int_{M}\left\langle P^{\mathrm{t}} E_{ \pm}^{\mathrm{t}} u, E_{\mp} f\right\rangle_{x} d \Omega_{g}=\int_{M}\left\langle u, E_{\mp} f\right\rangle_{x} d \Omega_{g},
\end{aligned}
$$


provided that $\operatorname{supp}\left(E_{ \pm}^{\mathrm{t}} u\right) \cap \operatorname{supp}\left(E_{\mp} f\right)$ is compact. It holds indeed that

$$
\operatorname{supp}\left(E_{ \pm}^{\mathrm{t}} u\right) \cap \operatorname{supp}\left(E_{\mp} f\right) \subset J_{ \pm}(\operatorname{supp} u) \cap J_{\mp}(\operatorname{supp} f),
$$

where the latter set is compact by Theorem 2.1.4.

TheOREM 2.4.6. Let $D: \mathscr{E}(M, \mathscr{V}) \rightarrow \mathscr{E}(M, \mathscr{V})$ be a pre-normally hyperbolic operator and let $\widetilde{D}$ be as in Definition 2.4.2. Then

$$
S_{ \pm}:=\widetilde{D} E_{ \pm}
$$

are unique advanced/retarded propagators for $D$, where $\widetilde{D}$ is such that $D \widetilde{D}$ is normally hyperbolic and $E_{ \pm}$are the respective advanced/retarded propagator for $D \widetilde{D}$.

Proof. The operator $D \widetilde{D}$ being normally hyperbolic, by Theorem 2.4.4, there exist unique advanced and retarded propagators $E_{ \pm}: \mathscr{E}(M, \mathscr{V}) \rightarrow \mathscr{E}(M, \mathscr{V})$ for $D \widetilde{D}$ and $E_{ \pm}^{\prime}: \mathscr{E}\left(M, \mathscr{V}^{*}\right) \rightarrow \mathscr{E}\left(M, \mathscr{V}^{*}\right)$ for $D^{\mathrm{t}} \widetilde{D}^{\mathrm{t}}$. Define

$$
S_{ \pm}:=\widetilde{D} E_{ \pm}, \quad S_{ \pm}^{\prime}:=\widetilde{D}^{\mathrm{t}} E_{ \pm}^{\prime} .
$$

We will show that $S_{ \pm}$are unique retarded/advanced propagators for $D$. By construction, on compactly supported sections we have

$$
\begin{aligned}
& D S_{-}=D \widetilde{D} E_{-}=\mathrm{id}, \\
& D^{\mathrm{t}} S_{+}^{\prime}=D^{\mathrm{t}} \widetilde{D}^{\mathrm{t}} E_{+}^{\prime}=\mathrm{id} .
\end{aligned}
$$

For all $f \in \mathscr{D}(M, \mathscr{V})$,

$$
\operatorname{supp}\left(S_{-} f\right)=\operatorname{supp}\left(\widetilde{D} E_{-} f\right) \subset \operatorname{supp}\left(E_{-} f\right) \subset J_{+}(\operatorname{supp} f)
$$

where the last inclusion follows from the support property of $E_{-}$. We have to show $S_{-} D=$ id. For arbitrary $u \in \mathscr{D}\left(M, \mathscr{V}^{*}\right), f \in \mathscr{D}(M, \mathscr{V})$,

$$
\begin{aligned}
\int_{M}\left\langle S_{ \pm}^{\prime} u, f\right\rangle_{x} d \Omega_{g} & =\int_{M}\left\langle S_{ \pm}^{\prime} u, D S_{\mp} f\right\rangle_{x} d \Omega_{g} \\
& =\int_{M}\left\langle D^{\mathrm{t}} S_{ \pm}^{\prime} u, S_{\mp} f\right\rangle_{x} d \Omega_{g}=\int_{M}\left\langle S_{-} u, f\right\rangle_{x} d \Omega_{g} .
\end{aligned}
$$

In the second equality, we have used that

$$
\operatorname{supp}\left(S_{+}^{\prime} u\right) \cap \operatorname{supp}\left(S_{-} f\right) \subset J_{-}(\operatorname{supp} u) \cap J_{+}(\operatorname{supp} f),
$$

where the latter set is compact by Theorem 2.1.4. We found $S_{-}=\left(S_{+}^{\prime}\right)^{\mathrm{t}}$. Taking the adjoint of $D^{\mathrm{t}} S_{+}^{\prime}=\mathrm{id}$, we get id $=\left(S_{+}^{\prime}\right)^{\mathrm{t}} D=D S_{-}$as requested.

Uniqueness can be seen as follows. Let $T_{-}$be another retarded propagator for $D$. Then, (2.4.2) holds with $S_{-}$replaced by $T_{-}$(and $S_{+}^{\prime}$ unchanged), hence $T_{-}=S_{-}$. The claim for the advanced propagator follows by analogous arguments.

2.4.2. Causal propagator. If a differential operator $D: \mathscr{E}(M, \mathscr{V}) \rightarrow \mathscr{E}(M, \mathscr{V})$ has an advanced and a retarded propagator, respectively $S_{+}$and $S_{-}$, then the causal propagator is defined as their difference

$$
S:=S_{+}-S_{-} .
$$


Its Schwartz kernel (if exists) is called the commutator function or Pauli-Jordan function (although it is actually a distribution). It plays an important role in quantization and first of all, it is essential in the description of smooth solutions of $D$.

The next theorem is a formal generalization of a result by Dimock [Dim82], which characterizes spaces of smooth solutions of $D$.

Theorem 2.4.7. Let $(M, g)$ be a globally hyperbolic spacetime, $\mathscr{V}$ a vector bundle over $M$ and let $D: \mathscr{E}(M, \mathscr{V}) \rightarrow \mathscr{E}(M, \mathscr{V})$ be a differential operator. Assume it has advanced/retarded propagators $S_{ \pm}$and let $S=S_{+}-S_{-}$be the corresponding causal propagator. Then:

(1) If $f \in \mathscr{D}(M, \mathscr{V})$ and $S f=0$, then there exists $g \in \mathscr{D}(M, \mathscr{V})$ s.t. $f=D g$.

(2) If $f \in \operatorname{Sol}_{\mathrm{sc}}(D)$ then there exists $g \in \mathscr{D}(M, \mathscr{V})$ s.t. $f=S g$.

Proof. (1): By assumption $S_{+} f=S_{-} f$. Setting $g:=S_{+} f=S_{-} f$ we see that

$$
\operatorname{supp} g \subset \operatorname{supp}\left(S_{+} f\right) \cap \operatorname{supp}\left(S_{-} f\right) \subset J_{+}(\operatorname{supp} f) \cap J_{-}(\operatorname{supp} f),
$$

hence $g \in \mathscr{D}(M, \mathscr{V})$. Moreover, $D g=D S_{+} f=f$.

(2): We can decompose $f=f_{+}-f_{-}$, where $\operatorname{supp} f_{ \pm} \subset J_{ \pm}(K)$ and $K$ is some compact subset of $M$. Then $g:=D f_{+}=D f_{-}$satisfies supp $g \subset J_{+}(K) \cap J_{-}(K)$, hence $g \in \mathscr{D}(M, \mathscr{V})$. Let us show that $S_{ \pm} g=f_{ \pm}$. Indeed, for all $u \in \mathscr{D}\left(M, \mathscr{V}^{*}\right)$ we have by Lemma 2.4 .5

$$
\begin{aligned}
\int_{M}\left\langle u, S_{ \pm} D f_{ \pm}\right\rangle_{x} d \Omega_{g} & =\int_{M}\left\langle S_{\mp}^{\mathrm{t}} u, D f_{ \pm}\right\rangle_{x} d \Omega_{g} \\
& =\int_{M}\left\langle D^{\mathrm{t}} S_{\mp}^{\mathrm{t}} u, f_{ \pm}\right\rangle_{x} d \Omega_{g}=\int_{M}\left\langle u, f_{ \pm}\right\rangle_{x} d \Omega_{g},
\end{aligned}
$$

where in the second equality we used that the set

$$
\operatorname{supp} f_{ \pm} \cap \operatorname{supp}\left(S_{\mp}^{\mathrm{t}} u\right) \subset J_{+}(K) \cap J_{-}(\operatorname{supp} u)
$$

is compact. Now $S_{ \pm} g=f_{+}$entails that $S g=S_{+} g-S_{-} g=f_{+}-f_{-}=f$.

From (2) it follows that using the map $S$ one can identify $\operatorname{Sol}_{\mathrm{sc}}(D)$ with the quotient space $\mathscr{D}(M, \mathscr{V}) / \operatorname{Ker} S$.

2.4.3. The charge form. Recall that $\overline{\mathscr{V}}^{*}$ is the anti-dual bundle, which fiberwise consists of anti-linear functionals on $\mathbb{C}$. We will consider sesquilinear forms on $\mathscr{V}_{x}$ smoothly depending on the base point $x \in M$. These can be defined as smooth sections of the bundle $\mathscr{L}\left(\mathscr{V}, \overline{\mathscr{V}}^{*}\right)$.

Let us fix a section $\beta \in \mathscr{E}\left(M, \mathscr{L}\left(\mathscr{V}, \overline{\mathscr{V}}^{*}\right)\right)$. Then for each $f, g \in \mathscr{D}(M, \mathscr{V})$ such that $\operatorname{supp} f \cap \operatorname{supp} g$ is compact,

$$
\int_{M} \beta(f, g) d \Omega_{g}=\int_{M}\langle\beta g, f\rangle_{x} d \Omega_{g}<\infty .
$$

Definition 2.4.8. Let $\beta \in \mathscr{E}\left(M, \mathscr{L}\left(\mathscr{V}, \overline{\mathscr{V}}^{*}\right)\right)$ be fiberwise hermitian and nondegenerate and let $A: \mathscr{D}(M, \mathscr{V}) \rightarrow \mathscr{E}(M, \mathscr{V})$ be a linear operator. We say that 
$A^{\dagger}: \mathscr{D}(M, \mathscr{V}) \rightarrow \mathscr{E}(M, \mathscr{V})$ is the formal adjoint (with respect to $\beta$ ), if

$$
\int_{M} \beta(f, A g) d \Omega_{g}=\int_{M} \beta\left(A^{\dagger} f, g\right) d \Omega_{g}, \quad f, g \in \mathscr{D}(M, \mathscr{V}) .
$$

We say that $A$ is formally self-adjoint (with respect to $\beta$ ) if $A^{\dagger}=A$.

In the literature, one often uses the notation $f^{\dagger}:=\beta f$ (in the context of the Dirac equation), where $\beta$ is understood as a mapping $\mathscr{D}(M, D M) \rightarrow \mathscr{D}\left(M, \overline{D M}^{*}\right)$. Of course, this is not to be confused with the formal adjoint of an operator.

The data required to construct a sensible non-interacting field theory on a globally hyperbolic spacetime consists of:

(1) the spacetime $(M, g)$ itself,

(2) a finite-dimensional vector bundle $\mathscr{V}$ over $M$, equipped a smooth section $\beta \in$ $\mathscr{E}\left(M, \mathscr{L}\left(\mathscr{V}, \overline{\mathscr{V}}^{*}\right)\right)$ which has values in hermitian, non-degenerate sesquilinear forms,

(3) a differential operator $D: \mathscr{E}(M, \mathscr{V}) \rightarrow \mathscr{E}(M, \mathscr{V})$ which has advanced/retarded propagators and is formally self-adjoint with respect to $\beta$.

In the fermionic case, an additional positivity condition is required, as we will see.

A bundle $\mathscr{V}$ equipped with $\beta$ as in (2) is called a hermitian bundle. The notation $(M, \mathscr{V}, D)$ will be used to abbreviate the data (1-3) and we will call $(M, \mathscr{V}, D)$ a field theory triple. The point of view that the non-interacting field theory is determined by such triple is adopted from [BG11] (where the authors work with real quantities rather than complex ones), a further generalization of this concept for gauge field theories has been recently proposed in [HS12].

The first step in the construction of quantum fields is to assign to the above data a pre-unitary space (more precisely a unitary space in the fermionic case). As explained in Chapter 1, this (pre-) unitary space serves to construct the respective CCR or CAR $C^{*}$-algebra, defined in a different way in the neutral and charged case. The numerous possible representations of the CCRs/CARs are then be studied by considering states on the CCR/CAR $C^{*}$-algebra.

Definition 2.4.9. The charge form for the field theory triple $(M, \mathscr{V}, D)$ is defined by

$$
\langle Q f, g\rangle:=-\mathrm{i} \int_{M}\langle\beta f, S g\rangle_{x} d \Omega_{g}, \quad f, g \in \mathscr{D}(M, \mathscr{V}),
$$

where $S$ is the causal propagator for $D$.

In the more familiar 'form-like' notation, (2.4.3) means

$$
Q(f, g)=-\mathrm{i} \int \beta(f, S g) d \Omega_{g}, \quad f, g \in \mathscr{D}(M, \mathscr{V}) .
$$

Proposition 2.4.10. Let $Q$ be the charge form for the triple $(M, \mathscr{V}, D)$. Then $(\mathscr{D}(M, \mathscr{V}) / \operatorname{Ker} S, Q)$ is a pre-unitary space, i.e., $Q$ is a non-degenerate, sesquilinear hermitian form on $\mathscr{D}(M, \mathscr{V}) / \operatorname{Ker} S$. 
Proof. Non-degeneracy follows trivially from the non-degeneracy of $\beta$. To prove hermiticity, observe that for all $f, g \in \mathscr{D}(M, \mathscr{V})$,

$$
\begin{aligned}
\int_{M} \overline{\beta\left(f, S_{ \pm} g\right)} d \Omega_{g} & =\int_{M} \beta\left(S_{ \pm} g, f\right) d \Omega_{g}=\int_{M} \beta\left(S_{ \pm} g, D S_{\mp} f\right) d \Omega_{g} \\
& =\int_{M} \beta\left(D S_{ \pm} g, S_{\mp} f\right) d \Omega_{g}=\int_{M} \beta\left(g, S_{\mp} f\right) d \Omega_{g}
\end{aligned}
$$

where we used formal self-adjointness of $P$ to go from the first line to the second. It follows that

$$
\overline{Q(f, g)}=\mathrm{i} \overline{\int \beta(f, S g) d \Omega_{g}}=-\mathrm{i} \int \beta(g, S f) d \Omega_{g}=Q(g, f) .
$$

In the fermionic case, one needs additionally the charge form $Q$ to be positive. This imposes an additional condition on the triple $(M, \mathscr{V}, D)$, which is satisfied spefically for the Dirac equation, but turns out to be problematic for higher-spin fields, i.e. the Rarita-Schwinger equation. We will not discuss this problem further, instead we refer the reader to [BG11, HM12a].

In most typical examples, like the Klein-Gordon or Dirac equation, it is possible to write a formula for $Q$ which involves integration over an arbitrary smooth Cauchy surface $\Sigma$ rather than over the whole spacetime $M$. In what follows we derive these results in a unified and generalized way. Our method is particularly useful in the case of non-vanishing electromagnetic potentials.

First, we will need the following lemma, which is a version of Green's formula for first-order differential operators. The proof is a direct computation which makes use of Stokes' formula.

LEmma 2.4.11. Let $R$ be a differential operator of order no larger than oneand suppose it is formally self-adjoint with respect to $\beta$. For any smooth submanifold $V$ of $M$,

$$
\int_{V}[\beta(R v, w)-\beta(v, R w)] d \Omega_{g}=\int_{\partial V} \beta\left(v, \sigma_{R}^{1}(\tilde{n}) w\right) d s, \quad v, w \in \mathscr{E}\left(M, \mathbb{C}^{2}\right)
$$

whenever the supports of $v, w$ are such that the integrals converge, where $d s$ is the volume measure on $\partial V$ induced from the metric on $M$ and $\tilde{n}$ is the outward pointing unit normal vector field across $\partial V$.

Lemma 2.4.12. Let $\Sigma$ be a smooth space-like Cauchy hypersurface of $(M, g)$. Let $D: \mathscr{E}(M, \mathscr{V}) \rightarrow \mathscr{E}(M, \mathscr{V})$ be a differential operator which has advanced/retarded propagators and in local coordinates can be written as

$$
D=N_{\mu} M^{\mu}+L=M_{\mu} N^{\mu}+L
$$

where:

(1) $N_{\mu}, M^{\mu}$ are differential operators of order at most one, formally self-adjoint with respect to $\beta$; 
(2) $L$ is a differential operator of arbitrary order s.t.

$$
\int_{J_{ \pm}(\Sigma) \backslash \Sigma}[\beta(L v, w)-\beta(v, L w)] d \Omega_{g}=0
$$

for all $v, w \in \mathscr{E}(M, \mathscr{V})$ s.t. the sets $J_{ \pm}(\Sigma) \cap \operatorname{supp} w$ are compact.

Then the charge form for the couple $(D, \beta)$ is given by

$$
\mathrm{i} Q(f, g)=\int_{\Sigma}\left[\beta\left(\sigma_{M^{\mu}}(n) N_{\mu} S f, S g\right)-\beta\left(S f, \sigma_{N^{\mu}}(n) M_{\mu} S g\right)\right] d s
$$

for $f, g \in \mathscr{D}(M, \mathscr{V})$, where $n$ is the future-pointing normal unit vector field across $\Sigma$. Proof. Let $V$ be equal $\Sigma^{+}:=J_{+}(\Sigma) \backslash \Sigma$ or $\Sigma^{-}:=J_{-}(\Sigma) \backslash \Sigma$. Applying Lemma 2.4.11 several times and taking into account $(2)$ we get

$$
\begin{aligned}
& \int_{V}[\beta(D v, w)-\beta(v, D w)] d \Omega_{g} \\
& =\int_{V}\left[\beta\left(N_{\mu} M^{\mu} v, w\right)-\beta\left(M^{\mu} v, N_{\mu} w\right)+\beta\left(M^{\mu} v, N_{\mu} w\right)-\beta\left(v, M^{\mu} N_{\mu} w\right)\right] d \Omega_{g} \\
& =\int_{\partial V}\left[\beta\left(\sigma_{M^{\mu}}^{1}(n) N_{\mu} v, w\right)-\beta\left(v, \sigma_{N^{\mu}}^{1}(n) Q_{\mu} w\right)\right] d s .
\end{aligned}
$$

We will apply the above equality to the cases

$$
V=\Sigma^{ \pm}, \quad \partial V=\Sigma, \quad v=S f, \quad w=S_{\mp} g, \quad \tilde{n}=\mp n,
$$

where $n$ is the forward pointing unit normal vector field for the Cauchy surface $\Sigma$. Strictly speaking, $\partial V$ consists of $\Sigma$ and a part which will be irrelevant in formulae involving integration by parts as a consequence of the fact that the set $V \cap \operatorname{supp} w$ is compact in both cases. Using homogeneity of the symbols $\sigma_{M^{\mu}}^{1}, \sigma_{N^{\mu}}^{1}$ and the definition of the causal propagator $S=S_{+}-S_{-}$, we obtain for any $f, g \in \mathscr{D}(M, \mathscr{V})$

$$
\begin{aligned}
\mathrm{i} Q(f, g)= & \int_{M} \beta(f, S g) d \Omega_{g}=\int_{\Sigma^{-}} \beta\left(D S_{+} f, S g\right) d \Omega_{g}+\int_{\Sigma^{+}} \beta\left(D S_{-} f, S g\right) d \Omega_{g} \\
= & \int_{\Sigma}\left[\beta\left(\sigma_{M^{\mu}}^{1}(n) N_{\mu} S_{+} f, S g\right)+\beta\left(\sigma_{M^{\mu}}^{1}(-n) N_{\mu} S_{-} f, S g\right)\right. \\
& \left.\quad-\beta\left(S_{+} f, \sigma_{N^{\mu}}^{1}(n) M_{\mu} S g\right)-\beta\left(S_{-} f, \sigma_{N^{\mu}}^{1}(-n) M_{\mu} S g\right)\right] d s \\
= & \int_{\Sigma}\left[\beta\left(\sigma_{M^{\mu}}^{1}(n) N_{\mu} S_{+} f, S g\right)-\beta\left(\sigma_{M^{\mu}}^{1}(n) N_{\mu} S_{-} f, S g\right)\right. \\
& \left.\quad-\beta\left(S_{+} f, \sigma_{N^{\mu}}^{1}(n) M_{\mu} S g\right)+\beta\left(S_{-} f, \sigma_{N^{\mu}}^{1}(n) M_{\mu} S g\right)\right] d s \\
= & \int_{\Sigma}\left[\beta\left(\sigma_{M^{\mu}}^{1}(n) M_{\mu} S f, S g\right)-\beta\left(S f, \sigma_{N^{\mu}}^{1}(n) M_{\mu} S g\right)\right] d s . \quad \square
\end{aligned}
$$

Roughly speaking, the assumption on $L$ means that it is formally self-adjoint with respect to $\beta$ and involves no time derivative. In particular, a constant (real) mass term $L=m^{2}$ trivially satisfies (2). 
2.4.4. Cauchy problem. The pre-unitary space $(\mathscr{D}(M, \mathscr{V}) / \operatorname{Ker} S, Q)$ corresponding to the triple $(M, \mathscr{V}, D)$ is the only data required to perform the construction of non-interacting quantum fields, both neutral and charged ones. The crucial property of $D$ used so far is the existence of advanced/retarded propagators and its formal self-adjointness with respect to a given hermitian form $\beta$. As pointed out in [BG11], it is a priori not even necessary to require that the Cauchy problem for $D$ is well posed. However, we will deal in the sequel only with operators with a well-posed Cauchy problem, and this property will be used in our analysis of quasi-free states later on.

Let us fix a Cauchy surface $\Sigma$ in a globally hyperbolic spacetime $(M, g)$ and let $n$ be the future directed unit normal vector field along $\Sigma$.

The proof of the well-posedness of the following non-characteristic Cauchy problem for normally hyperbolic differential operators can be found for instance in [BGP08].

Theorem 2.4.13. Let $P: \mathscr{E}(M, \mathscr{V}) \rightarrow \mathscr{E}(M, \mathscr{V})$ be normally hyperbolic and let $\Sigma$ be Cauchy surface. Then for initial data $\varphi_{0}, \varphi_{1} \in \mathscr{D}\left(\Sigma,\left.\mathscr{V}\right|_{\Sigma}\right)$ the Cauchy problem

$$
(P) \quad\left\{\begin{array}{l}
P f=0, \quad f \in \mathscr{E}(M, \mathscr{V}) \\
\left.f\right|_{\Sigma}=\varphi_{0}, \\
\left.\mathrm{i}^{-1} n^{\mu} \nabla_{\mu}\right|_{\Sigma}=\varphi_{1}
\end{array}\right.
$$

has a unique solution $f$ and this solution satisfies $\operatorname{supp} f \subset J\left(\operatorname{supp} \varphi_{0} \cup \operatorname{supp} \varphi_{1}\right)$.

In the case when external electromagnetic potentials are present, it is more appropriate to take $\left.\mathrm{i}^{-1} n^{\mu}\left(\nabla_{\mu}+\mathrm{i} A_{\mu}\right)\right|_{\Sigma}=\varphi_{1}$ as initial datum. The analogue of Theorem 2.4.13 for such modified Cauchy problem can be proved by reinterpreting $\nabla_{\mu}^{A}:=$ $\nabla_{\mu}+\mathrm{i} A_{\mu}$ as the covariant derivative in the sense of principal bundles. There will be however no need of doing so, as this claim will also follow as a side result of our results for pre-normally hyperbolic operators.

The next theorem is a straightforward generalization of a result Mühlhoff, proved initially for first-order pre-normally hyperbolic operators [Müh11]. The validity of the proof relies on an assumption on the symbol, which is automatically satisfied for pre-normally hyperbolic operators of order one.

TheOrem 2.4.14. Let $D, \widetilde{D}: \mathscr{E}(M, \mathscr{V}) \rightarrow \mathscr{E}(M, \mathscr{V})$ be differential operators such that $D \widetilde{D}$ is normally hyperbolic and suppose $\sigma_{D}^{k}(n)=\sigma_{\widetilde{D}}^{k}(n)=0$ for $k \geq 2$. Let $\Sigma \subset M$ be a smooth spacelike Cauchy surface. Then the Cauchy problem

$$
(D) \quad\left\{\begin{array}{l}
D f=0, \quad f \in \mathscr{E}(M, \mathscr{V}) \\
\left.f\right|_{\Sigma}=\varphi_{0}
\end{array}\right.
$$

has a unique solution for every initial datum $\varphi_{0} \in \mathscr{D}\left(\Sigma,\left.\mathscr{V}\right|_{\Sigma}\right)$. This solution satisfies $\operatorname{supp} f \subset J\left(\operatorname{supp} \varphi_{0}\right)$.

Theorem 2.4.14 is a direct consequence of (2) and (3) of the following lemma.

Lemma 2.4.15. Let $D, \widetilde{D}$ be as in Theorem 2.4.14 and consider the following two 
Cauchy problems:

$$
\begin{aligned}
& (\widetilde{D} D)\left\{\begin{array} { l } 
{ \widetilde { D } D f = 0 , } \\
{ f | _ { \Sigma } = \varphi _ { 0 } , } \\
{ \mathrm { i } ^ { - 1 } n ^ { \mu } \nabla _ { \mu } | _ { \Sigma } = \varphi _ { 1 } , }
\end{array} \quad ( \widetilde { D } D ) ^ { \prime } \quad \left\{\begin{array}{l}
\widetilde{D} D f=0, \\
\left.f\right|_{\Sigma}=\varphi_{0}, \\
\left.(D f)\right|_{\Sigma}=\varphi_{1},
\end{array}\right.\right. \\
& \text { given } \varphi_{0}, \varphi_{1} \in \mathscr{D}\left(\Sigma,\left.\mathscr{V}\right|_{\Sigma}\right) ; \quad \text { given } \varphi_{0} \in \mathscr{D}\left(\Sigma,\left.\mathscr{V}\right|_{\Sigma}\right) \text {. }
\end{aligned}
$$

The following statements hold true:

(1) If $f \in \mathscr{E}(M, \mathscr{V})$ solves $(\widetilde{D} D)^{\prime}$ for initial datum $\varphi_{0}$, then $f$ solves $(\widetilde{D} D)^{\prime}$ for initial data $\varphi_{0}$ and $\varphi_{1}:=\left.\nabla_{n} f\right|_{\Sigma}$.

(2) For any initial data $\varphi_{0}$ there exists a unique solution $f \in \mathscr{E}(M, \mathscr{V})$ to $(\widetilde{D} D)^{\prime}$. It satisfies $\operatorname{supp} f \subset J\left(\operatorname{supp} \varphi_{0}\right)$.

(3) $f \in \mathscr{E}(M, \mathscr{V})$ solves $(D)$ for initial datum $\varphi_{0}$ iff it solves $(\widetilde{D} D)^{\prime}$ with initial data $\varphi_{0}$ and $\varphi_{1}=0$.

Proof. (1): The only non-trivial part is to prove that $\left.\nabla_{n} f\right|_{\Sigma}$ is compactly supported. First, observe that the assumptions on $\sigma_{D}$ imply that $D=\sigma_{D}^{1}(n) \nabla_{n}+R$, where $R$ is a differential operator s.t. $\sigma_{R}^{k}(n)=0$ for all $k \geq 1$. Moreover, normal hyperbolicity implies that $\sigma_{D \widetilde{D}}^{2}(n)=\sigma_{D}^{1}(n) \sigma_{\widetilde{D}}^{1}(n)$ is invertible. In particular, $\sigma_{D}^{1}(n)$ is invertible. Therefore, the equality

$$
0=\left.(D f)\right|_{\Sigma}=\left.\sigma_{D}^{1}(n) \nabla_{n} f\right|_{\Sigma}+\left.R f\right|_{\Sigma}
$$

is equivalent to

$$
\left.\nabla_{n} f\right|_{\Sigma}=-\left.\left(\sigma_{D}^{1}(n)\right)^{-1} R f\right|_{\Sigma},
$$

where the RHS is compactly supported.

(2): Given $\varphi_{0}$, let us define

$$
\varphi_{1}:=-\left(\sigma_{D}^{1}(n)\right)^{-1} R \varphi_{0} \in \mathscr{D}\left(\Sigma,\left.\mathscr{V}\right|_{\Sigma}\right),
$$

where $R$ is as in 2.4.7. By Theorem 2.4.4, there exists a unique solution $f \in \mathscr{E}(M, \mathscr{V})$ of $(\widetilde{D} D)$ with initial data $\varphi_{0}, \varphi_{1}$ and it satisfies the requested support properties. According to the discussion in the proof of $(1), \varphi_{1}$ is chosen in such way that $\left.(D f)\right|_{\Sigma}=$ 0 , therefore $f$ is a solution of $(\widetilde{D} D)^{\prime}$ with initial datum $\varphi_{0}$. Uniqueness follows from (1) and uniqueness of solutions of $(\widetilde{D} D)$.

(3): Clearly, if $f$ solves $(D)$ with initial datum $\varphi_{0}$ then it also solves $(\widetilde{D} D)^{\prime}$ with the appropriate initial data. Conversely, let $f$ solves $(\widetilde{D} D)^{\prime}$. This implies $D \widetilde{D} D f=0$, $\left.(D f)\right|_{\Sigma}=0$ and $\left.(\widetilde{D} D f)\right|_{\Sigma}=0$. Observe that this means $D f$ solves a Cauchy problem analogous to $(\widetilde{D} D)^{\prime}$, but with the role of $\widetilde{D}$ and $D$ inversed, and vanishing initial data. By (2), solutions of this Cauchy problem are unique, hence $D f=0$ and $f$ solves $(D)$.

2.4.5. Cauchy-data charge form. The well-posedness of the Cauchy problem $(D)$ implies that the restriction map $\rho, \rho f=\left.f\right|_{\Sigma}$ is a bijection when understood as $\operatorname{map} \operatorname{Sol}_{\mathrm{sc}}(D) \rightarrow \mathscr{D}(\Sigma, \mathscr{V})$. 
The charge form $Q$ can be transported as follows to a form $q$ acting on Cauchy data.

DeFinition 2.4.16. Let $D$ be pre-normally hyperbolic and assume that the Cauchy problem $(D)$ is well-posed. The Cauchy-data charge form is defined by

$$
\begin{aligned}
& q: \mathscr{D}(\Sigma, \mathscr{V}) \rightarrow{\overline{\mathscr{D}(M, \mathscr{V})^{*}}}^{*}, \\
& q:=(\rho S)^{*} Q(\rho S),
\end{aligned}
$$

that is

$$
Q(f, g)=q(\rho S f, \rho S g), \quad f, g \in \mathscr{D}(M, \mathscr{V}) / \operatorname{Ker} S
$$

in the 'form-like' notation, where $S$ is the causal propagator for $D$.

It follows from Theorem 2.4.7 that $(\mathscr{D}(\Sigma, \mathscr{V}), q)$ is a pre-unitary space and we have an isomorphism

$$
(\mathscr{D}(M) / \operatorname{Ker} E, Q) \cong(\mathscr{D}(\Sigma, \mathscr{V}), q) .
$$

The explicit form of $q$ will be deduced from Lemma 2.4 .12 in the cases we are specifically interested in.

Observe that the dual $\rho^{*}: \mathscr{E}^{\prime}\left(\Sigma,\left.\overline{\mathscr{V}}^{*}\right|_{\Sigma}\right) \rightarrow \mathscr{E}^{\prime}(M, \mathscr{V})$ of the restriction map $\rho$ : $\mathscr{E}(M, \mathscr{V}) \rightarrow \mathscr{E}\left(\Sigma,\left.\mathscr{V}\right|_{\Sigma}\right)$ extends to an operator $\mathscr{D}\left(\Sigma,\left.\overline{\mathscr{V}}^{*}\right|_{\Sigma}\right) \rightarrow \mathscr{E}(M, \mathscr{V})$. We obtain a result which generalizes [Dim82, Prop 2.4].

Proposition 2.4.17. The unique solution of $(D)$ with initial datum $\varphi_{0}$ is given by

$$
f=-\mathrm{i} S \beta^{-1} \rho^{*} q \varphi_{0} \in \mathscr{E}(M, \mathscr{V})
$$

where $q$ is understood as an operator $\left.\left.\mathscr{D}\left(\Sigma,\left.\mathscr{V}\right|_{\Sigma}\right)\right) \rightarrow \mathscr{D}\left(\Sigma,\left.\overline{\mathscr{V}}^{*}\right|_{\Sigma}\right)\right)$.

Proof. By definition of $q$,

$$
\langle q \rho f, \rho S g\rangle=\mathrm{i}\langle\beta f, g\rangle
$$

Hence $f=-\mathrm{i} \beta^{-1} S^{*} \rho^{*} q \rho f$. By formal self-adjointness of $S$ w.r.t. $\beta$, this entails $f=-\mathrm{i} S \beta^{-1} \rho^{*} q \rho f$.

\subsection{The Dirac equation in external potentials}

The main motivation for introducing the notion of pre-normally hyperbolic operators is the study of the Dirac equation, possibly on curved spacetime or in external potentials. We briefly recall the relevant definitions and show how do they fit in the framework developped in the previous section. A broader introduction to spin structures and the Dirac equation on curved spacetime can be found in the references [Dim82, San08, Hac10].

In order to treat background electromagnetic fields, it is often useful to use the language of $U(1)$-principle bundles and write the Dirac operator using the corresponding covariant derivative. Such approach is particularly fruitful in investigating issues like local covariance, as studied in [Zah13], and has the advantage of generalizing to non-abelian groups. We restrain from doing so, as we will be more interested in analytical properties of the corresponding Dirac operator. 
2.5.1. Spin structures. Let us denote $S O_{0}(1, d)$ the restricted Lorentz group, i.e., the connected component of the Lie group $S O(n-1,1)$ which contains the identity element.

The Lorentz frame bundle, denoted $L M$, is a principle fiber bundle with group structure $S O_{0}(1, d)$.

The universal covering group of $S O_{0}(1, d)$ is denoted $\operatorname{Spin}_{0}(1, d)$. Let us denote $\Pi: \operatorname{Spin}_{0}(1, d) \rightarrow S O_{0}(1, d)$ the double-covering Lie group homomorphism (in the case $n=4$ it actually concides with the universal covering homomorphism).

DEFINITION 2.5.1. A spin structure $S M$ is a principal fibre bundle with base manifold $M$ and structure group $\operatorname{Spin}_{0}(1, d)$, equipped with a smooth bundle homomorphism $\theta: S M \rightarrow L M$ s.t.

(1) $\theta$ preserves the base points, i.e., $\pi_{L} \circ \theta=\pi_{S}$, where $\pi_{L}, \pi_{S}$ are the respective base projections of $L M, S M$;

(2) $\theta$ satisfies

$$
R_{L}(\Lambda) \circ \theta=\theta \circ R_{S}(\Pi(\Lambda)), \quad \Lambda \in \operatorname{Spin}_{0}(1, d)
$$

where $R_{S}, R_{L}$ are the respective right actions of the structure group on $L M$, $S M$.

There are well-known criteria for the existence of a spin structure on a spacetime, it suffices for instance that $M$ is parallelizable, i.e. admits a global Lorentz frame (see, e.g. [Hus94]). This is the case in particular if $M$ is globally hyperbolic and $n=4$, or if $M$ is flat and $n$ is arbitrary. Another feature of parallelizable spacetimes is that the bundles $L M$ and $S M$ are trivial (for any choice of spin structure).

\subsubsection{Clifford relations and spinor bundles.}

Definition 2.5.2. Let $V$ be a finite-dimensional vector space over $\mathbb{R}$ or $\mathbb{C}$ and let $\eta$ be the Lorentzian scalar product on $\mathbb{R}^{1, d}$. A linear map $\gamma^{\pi}: \mathbb{R}^{1, d} \rightarrow L(V)$ is a representation of the Clifford relations in $V$ if

$$
\left\{\gamma^{\pi}\left(e_{\mu}\right), \gamma^{\pi}\left(e_{\nu}\right)\right\}=2 \eta_{\mu \nu} \mathbf{1}, \quad \mu, \nu=0,1, \ldots, d .
$$

This definition can be generalized in several ways, for instance by replacing Minkowski space with some other space equipped with a symmetric bilinear form or by considering vector spaces over other fields than $\mathbb{R}$. This more general framework is studied in [DG13] and is somehow reminescent of the definition of CAR representations introduced in Chapter 1.

We will focus here only on representations of the Cliffords relations for $\eta$ in $n=3,4,9,10 \bmod 8$ spacetime dimensions. In such case there exists a Majorana representation $\gamma^{\pi}$ in $\mathbb{C}^{2^{[n / 2]}}$, i.e. a representation of the Clifford relations s.t.

$$
\left(\gamma^{\pi}\left(e_{0}\right)\right)^{*}=\gamma^{\pi}\left(e_{0}\right), \quad\left(\gamma^{\pi}\left(e_{k}\right)\right)^{*}=-\gamma^{\pi}\left(e_{k}\right) \quad(k=1, \ldots, d), \quad \overline{\gamma^{\pi}\left(e_{\mu}\right)}=-\gamma^{\pi}\left(e_{\mu}\right),
$$

where the bar stands for entry-wise complex conjugation (and $[n / 2]$ stands for the entire part of $n / 2)$. A well-known result states that there exists a representation $\varrho: \operatorname{Spin}_{0}(1, d) \rightarrow L\left(\mathbb{C}^{2^{[n / 2]}}\right)$ whose image is the $\mathbb{R}$-linear span of $\left\{\gamma^{\pi}\left(e_{\mu}\right)\right\}_{\mu=0}^{d}$. 
Definition 2.5.3. Let $S M$ be a spin structure over $M$. The bundle of Majorana spinors $D M$ is the vector bundle associated to $S M$ and the representation $\varrho: \operatorname{Spin}_{0}(1, d) \rightarrow \mathbb{C}^{2^{[n / 2]}}$, i.e.

$$
D M=S M \ltimes_{\varrho} \mathbb{C}^{2^{[n / 2]}} .
$$

A review on spinor bundles in arbitrary dimensions can be found for instance in [San08]. As the results we present later on do not depend in an essential way on the assumption $n=3,4,9,10 \bmod 8$ (cf. the remark in [SV00, Sec 3.4]), we will not remind it anymore. The dual bundle of $D M$ is traditionally called the cospinor bundle.

If $E$ is a local section of $S M$, it induces via $\theta$ a local section of $S M$, whose components will be denoted $e_{\mu}$ (not to be confused with the basis in $\mathbb{R}^{1, d}$ ). It also induces a set local sections $\left\{E_{A}\right\}_{A=0}^{2^{[n / 2]}}$ of $D M$ defined by $E_{A}=\left[E, s_{A}\right]$, where $s_{A}$ is the standard basis in $\mathbb{C}^{2^{[n / 2]}}$. Moreover, we can choose local sections $e^{\mu}, E^{A}$ in the dual bundles of $S M$ and $D M$, such that $e^{\mu}\left(e_{\nu}\right)=\delta_{\nu}^{\mu}$ and $E^{A}\left(E_{B}\right)=\delta_{B}^{A}$.

2.5.3. Dirac operator. Assume that we are given a spin structure $S M$.

The Levi-Civita connection $\nabla$ on $(M, g)$ induces a connection on the frame bundle $L M$ and since the Lie algebras of $S O_{0}(1, d)$ and $\operatorname{Spin}_{0}(1, d)$ can be identified, this also induces a connection on $S M$. This in turn yields a connection on the spinor bundle $D M$. The covariant derivative of this connection will also be denoted $\nabla$ : $\mathscr{E}(M, T M \otimes D M) \rightarrow \mathscr{E}(M, D M)$ for sake of shortness of notation. If $f=f^{A} E_{A}$ is a local section in $D M$ then $\nabla f=\nabla_{\mu} f^{A}\left(e^{\mu} \otimes E_{A}\right)$ has components

$$
\nabla_{\mu} f^{A}=\partial_{\mu} f^{A}+\varsigma_{\mu}{ }^{A}{ }_{B} f^{B},
$$

where

$$
\partial_{\mu} f^{A}=d f^{A}\left(e_{\mu}\right), \quad \varsigma_{\mu}^{A}{ }_{B}=-\frac{1}{4} \Gamma_{\mu \nu}^{\lambda} \gamma_{\lambda}^{A}{ }_{C} \gamma^{\nu C_{B}},
$$

and $\Gamma_{\mu \nu}^{\lambda}$ are Christoffel's symbols of the Levi-Civita connection.

The spinor tensor $\gamma$ is by definition the element of $\mathscr{E}\left(M, T^{*} M \otimes D M \otimes(D M)^{*}\right)$ whose components $\left(\gamma_{\mu}\right)_{B}^{A}$ in the induced frame $e^{\mu} \otimes E_{A} \otimes E^{B}$ are equal to the matrix elements $\gamma^{\pi}\left(e_{\mu}\right)^{A}{ }_{B}$. One can show that this definition does not depend on the choice of the local section $E$.

The Dirac operator in a background potential $A=A_{\mu} d x^{\mu}$ and with mass term $m$ is defined by

$$
D=-\gamma^{\mu}\left(\nabla_{\mu}+\mathrm{i} A_{\mu}\right)+m
$$

Clearly, this definition is independent on the choice of a local frame and yields a differential operator $\mathscr{E}(M, D M) \rightarrow \mathscr{E}(M, D M)$ of order one.

Let us also define an auxiliary differential operator $\widetilde{D}$ by

$$
\widetilde{D}=-\gamma^{\mu}\left(\nabla_{\mu}-\mathrm{i} A_{\mu}\right)-m
$$

Then we can compute as in [Zah13]

$$
D \widetilde{D}=-\nabla_{\mu} \nabla^{\mu}+\frac{R}{4}+\frac{\mathrm{i}}{4}\left[\gamma^{\mu}, \gamma^{\nu}\right] F_{\mu \nu}+m^{2}-\gamma^{\nu} \partial_{\mu} m
$$


where $F_{\mu \nu}=\partial_{\mu} A_{\nu}-\partial_{\nu} A_{\mu}$ and $R$ is the scalar curvature of $(M, g)$ (i.e., the trace of the Ricci tensor). Therefore, $D$ is pre-normally hyperbolic.

The Dirac conjugation is the hermitian form $\beta \in \mathscr{E}\left(M, D M \otimes \overline{D M}^{*}\right)$ defined by

$$
\langle\beta f, g\rangle:=-\mathrm{i}\left\langle\bar{f}, \gamma_{0} g\right\rangle, \quad f, g \in \mathscr{D}(M, D M) .
$$

A well-known result states that $D$ is formally self-adjoint w.r.t. $\beta$. Thus, we have a field theory triple $(M, D M, D)$ with hermitian structure $\beta$ and an associated charge form $Q$.

Proposition 2.5.4. The Cauchy-data charge form for the triple $(M, D M, D)$ is given by

$$
q=-\mathrm{i} \beta \gamma_{\mu} n^{\mu}
$$

Proof. It suffices to apply Lemma 2.4.12 with $N_{\mu}=-\gamma_{\mu}$ and $M^{\mu}=\nabla_{\mu}$.

The formula (2.5.1) can be then used to prove positivity of $q$, and hence of the charge form $Q$.

\subsection{Two-component form of the Klein-Gordon equation}

As realized a long time ago by Villars [FV58], the Klein-Gordon equation on Minkowski space is equivalent to a differential equation which involves only one time derivative. The same construction is possible on a class of curved spacetimes and in the presence of external potentials, and we will show that it corresponds to a pre-normally hyperbolic operator with many properties quite analogous to the Dirac equation.

As previously, let us fix a real-valued external potential $A=A_{\mu} d x^{\mu}$ and a realvalued smooth section $m$ and consider the Klein-Gordon operator

$$
\begin{aligned}
P & =-\left(\nabla_{\mu}+\mathrm{i} A_{\mu}\right)\left(\nabla^{\mu}+\mathrm{i} A^{\mu}\right)+m^{2} \\
& =-\nabla_{\mu}^{A} \nabla^{A \mu}+m^{2},
\end{aligned}
$$

where we denoted $\nabla_{\mu}^{A}:=\left(\nabla_{\mu}+\mathrm{i} A_{\mu}\right)$.

Let us assume in addition that the spacetime is foliated by a family of Cauchy surfaces $\left\{\Sigma_{s}\right\}_{s \in \mathbb{R}}$, such that the vector field $\partial_{s}=n^{\mu} \partial_{\mu}$ is geodesic and orthonormal to the Cauchy surfaces, i.e.,

$$
\nabla_{\nu} n_{\mu}-\nabla_{\mu} n_{\nu}=0, \quad n^{\mu} \nabla_{\mu} n^{\nu}=0 .
$$

Such 'Gaussian foliation' can be always constructed in a neighbourhood of a Cauchy surface, thus our results will be also valid on a generic spacetime after possibly restricting to a small enough region which contains a given Cauchy surface.

The metric $g_{\mu \nu}$ induces a $d$-dimensional Riemannian metric $h_{\mu \nu}$ by the formula

$$
h_{\mu \nu}=g_{\mu \nu}+n_{\mu} n_{\nu} .
$$

Let us now consider the trivial bundle with base manifold $M$ and fiber $\mathbb{C}^{2}$ and 
define a differential operator $D: \mathscr{D}\left(M, \mathbb{C}^{2}\right) \rightarrow \mathscr{D}\left(M, \mathbb{C}^{2}\right)$ by

$$
D=\left(\begin{array}{cc}
\mathrm{i}^{-1} n^{\mu} \nabla_{\mu}^{A} & \mathbf{1} \\
-h^{\mu \nu} \nabla_{\mu}^{A} \nabla_{\nu}^{A}+m^{2} & \mathrm{i}^{-1} n^{\mu} \nabla_{\mu}^{A}
\end{array}\right),
$$

We will refer to $D$ as the two-component Klein-Gordon operator. The two-component Klein-Gordon equation is related to $P$ as follows. Writing $f=\left(f_{0}, f_{1}\right)$ for $f \in$ $\mathscr{E}\left(M, \mathbb{C}^{2}\right)$, we have

$$
D f=0 \Longleftrightarrow\left\{\begin{array}{l}
P g=0, \\
f_{0}=g, \quad f_{1}=\mathrm{i}^{-1} n^{\mu} \nabla_{\mu}^{A} g .
\end{array}\right.
$$

Let us check that $D$ is pre-normally hyperbolic. Indeed, defining an auxiliary differential operator

$$
\widetilde{D}=\left(\begin{array}{cc}
\mathrm{i} n^{\mu} \nabla_{\mu}^{A} & \mathbf{1} \\
-h^{\mu \nu} \nabla_{\mu}^{A} \nabla_{\nu}^{A}+m^{2} & \mathrm{i} n^{\mu} \nabla_{\mu}^{A}
\end{array}\right)
$$

we obtain that

$$
D \widetilde{D}=\widetilde{D} D=\left(\begin{array}{ll}
P & 0 \\
0 & P
\end{array}\right)
$$

which is clearly normally hyperbolic.

As a consequence, $D$ possesses unique advanced/retarded propagators $S_{ \pm}=\widetilde{D} E_{ \pm}$, where $E_{ \pm}$are the advanced/retarded propagators for $P$ (cf. Theorem 2.4.6). The causal propagators are related by $S=\widetilde{D} E$.

Moreover, from Theorem 2.4.14 we obtain that the Cauchy problem $(D)$ is wellposed. Indeed, the assumption $\sigma_{D}^{2}(n)=\sigma_{\widetilde{D}}^{2}(n)=0$ is easy to check using (2.6.2).

2.6.1. Charge form. Let us equip $\mathbb{C}^{2}$ with the hermitian form

$$
\beta(w, z):=\bar{w}_{0} z_{1}+\overline{w_{1}} z_{0} \quad w=\left(\begin{array}{l}
w_{0} \\
w_{1}
\end{array}\right), z=\left(\begin{array}{c}
z_{0} \\
z_{1}
\end{array}\right) \in \mathbb{C}^{2},
$$

A straightforward computation shows that $D$ is formally self-adjoint with respect to $\beta$. Therefore, if we equip the trivial bundle $\mathbb{C}^{2}$ with base space $M$ with the hermitian form $\beta,\left(M, \mathbb{C}^{2}, D\right)$ is a field theory triple and consequently if the assumptions of Proposition 2.4.10 are satisfied, the charge form $Q$ is a well-defined non-degenerate hermitian form and we have a pre-unitary space

$$
\left(\mathscr{D}\left(M, \mathbb{C}^{2}\right) / \operatorname{Ker} S, Q\right) .
$$

Since the Cauchy problem $(D)$ is well-posed, we also have a Cauchy-surface charge form.

Proposition 2.6.1. The Cauchy-data charge form for the triple $\left(M, \mathbb{C}^{2}, D\right)$ equals

$$
q(f, g)=\int_{\Sigma}\left(\bar{f}_{0} g_{1}+\bar{f}_{1} g_{0}\right) d s, \quad f=\left(\begin{array}{l}
f_{0} \\
f_{1}
\end{array}\right), g=\left(\begin{array}{l}
g_{0} \\
g_{1}
\end{array}\right) \in \mathscr{D}\left(\Sigma, \mathbb{C}^{2}\right),
$$

Proof. We apply Lemma 2.4.12 with

$$
N_{\mu}=\left(\begin{array}{cc}
n_{\mu} & 0 \\
0 & n_{\mu}
\end{array}\right), \quad M_{\mu}=\left(\begin{array}{cc}
\mathrm{i}^{-1} \nabla_{\mu}^{A} & 0 \\
0 & \mathrm{i}^{-1} \nabla_{\mu}^{A}
\end{array}\right),
$$




$$
L=\left(\begin{array}{cc}
0 & \mathbf{1} \\
-h^{\mu \nu} \nabla_{\mu}^{A} \nabla_{\nu}^{A}+m^{2} & 0
\end{array}\right) .
$$

Since $\sigma_{L}^{2}\left(n^{\mu}\right)=\sigma_{L}^{1}\left(n^{\mu}\right)=0$ we deduce that $L$ satisfies assumption (2) of Lemma 2.4.12 indeed.

2.6.2. Equivalence with Klein-Gordon equation. Let us now consider the original Klein-Gordon equation again. First, observe that the relation with the twocomponent form (2.6.3) implies that the following Cauchy problem in external potentials is well-posed.

Corollary 2.6.2. Let $P$ be the Klein-Gordon operator in external potentials and fix a Cauchy surface $\Sigma$. For any $\varphi_{0}, \varphi_{1} \in \mathscr{D}\left(\Sigma,\left.\mathscr{V}\right|_{\Sigma}\right)$ the Cauchy problem

$$
(K G) \quad\left\{\begin{array}{l}
P f=0, \quad f \in \mathscr{E}(M, \mathscr{V}) \\
\left.f\right|_{\Sigma}=\varphi_{0}, \\
\left.\mathrm{i}^{-1} n^{\mu}\left(\nabla_{\mu}+\mathrm{i} A_{\mu}\right)\right|_{\Sigma}=\varphi_{1}
\end{array}\right.
$$

has a unique solution $f$ and this solution satisfies $\operatorname{supp} f \subset J\left(\operatorname{supp} \varphi_{0} \cup \operatorname{supp} \varphi_{1}\right)$.

Obviously, the complex vector space $\mathbb{C}$ has a non-degenerate, hermitian sesquilinear form $z \mapsto \bar{z} \in \mathbb{C}^{*}$, which we will denote $\beta_{\mathrm{KG}}$ for the sake of consistency of notation. The Klein-Gordon operator $P$ is formally self-adjoint with respect to this hermitian form (thanks to the assumption that $A_{\mu}$ and $m^{2}$ are real). We have thus a charge form $Q_{\mathrm{KG}}$ associated to the triple $(M, \mathbb{C}, P)$.

Let us now discuss the corresponding notions on a Cauchy surface $\Sigma$. The map

$$
\rho_{\mathrm{KG}}: \mathscr{E}\left(M, \mathbb{C}^{2}\right) \rightarrow \mathscr{E}\left(\Sigma, \mathbb{C}^{2}\right), \quad \rho_{\mathrm{KG}} f:=\left(\begin{array}{c}
\left.f\right|_{\Sigma} \\
\left.\mathrm{i}^{-1} n^{\mu}\left(\nabla_{\mu}+\mathrm{i} A_{\mu}\right) f\right|_{\Sigma}
\end{array}\right)
$$

assigns to a smooth solution its initial data, therefore it maps bijectively $\operatorname{Sol}_{\mathrm{sc}}(P) \rightarrow$ $\mathscr{D}\left(\Sigma, \mathbb{C}^{2}\right)$

Let $E$ be the causal propagator for $P$. If we define in analogy to the twocomponent Klein-Gordon equation

$$
\begin{aligned}
& q_{\mathrm{KG}}: \mathscr{D}\left(\Sigma, \mathbb{C}^{2}\right) \rightarrow\left(\mathscr{D}\left(\Sigma, \mathbb{C}^{2}\right)^{*}\right. \\
& q_{\mathrm{KG}}:=(\rho E)^{*-1} Q(\rho E)^{-1},
\end{aligned}
$$

then $q_{\mathrm{KG}}$ is a non-degenerate hermitian form on $\mathscr{D}\left(\Sigma, \mathbb{C}^{2}\right)$. We obtain this way isomorphic pre-unitary spaces

$$
\left(\mathscr{D}(M) / \operatorname{Ker} E, Q_{\mathrm{KG}}\right) \cong\left(\mathscr{D}\left(\Sigma, \mathbb{C}^{2}\right), q_{\mathrm{KG}}\right) .
$$

Proposition 2.6.3. The Cauchy-data charge form for the triple $(M, \mathbb{C}, P)$ equals

$$
q_{\mathrm{KG}}(f, g)=\int_{\Sigma}\left(\bar{f}_{0} g_{1}+\bar{f}_{1} g_{0}\right) d s \quad f=\left(\begin{array}{c}
f_{0} \\
f_{1}
\end{array}\right), g=\left(\begin{array}{c}
g_{0} \\
g_{1}
\end{array}\right) \in \mathscr{E}\left(\Sigma, \mathbb{C}^{2}\right),
$$

Proof. We apply Lemma 2.4.12 with $N_{\mu}=M_{\mu}=\mathrm{i}^{-1}\left(\partial_{\mu}+\mathrm{i} A_{\mu}\right)$ and $L=m^{2}$.

Thus, $q_{\mathrm{KG}}$ coincides with the Cauchy-data charge form $q$ for the two-component 
Klein-Gordon equation computed in Proposition 2.6.1. We can sum up this discussion as follows.

Corollary 2.6.4. We have the following isomorphisms of pre-unitary spaces:

$$
\left(\mathscr{D}(M) / \operatorname{Ker} E, Q_{\mathrm{KG}}\right) \stackrel{(\rho E)^{-1}}{\cong}\left(\mathscr{D}\left(\Sigma, \mathbb{C}^{2}\right), q\right) \stackrel{\rho S}{\cong}\left(\mathscr{D}\left(M, \mathbb{C}^{2}\right) / \operatorname{Ker} S, Q\right) .
$$

This result means that the conventional approach to quantization based on the Klein-Gordon operator $P$ is equivalent to the approach which makes use of the twocomponent form $D$.

\subsection{Distributional two-point functions}

Let us now discuss quasi-free states on the $C^{*}$-algebras

$$
\operatorname{CAR}(\mathscr{D}(M, \mathscr{V}) / \operatorname{Ker} S, Q), \quad \operatorname{CCR}(\mathscr{D}(M, \mathscr{V}) / \operatorname{Ker} S, Q)
$$

where $Q$ is the charge form associated to a field theory triple $(M, \mathscr{V}, D)$, i.e.,

$$
Q(f, g)=-\mathrm{i} \int_{M} \beta(f, S g) d \Omega_{g}, \quad f, g \in \mathscr{D}(M, \mathscr{V}) .
$$

If $\Lambda$ is the two-point function of a quasi-free state $\omega$ and it maps continuously $\mathscr{D}(M, \mathscr{V})$ to its topological anti-dual, then by Schwarz kernel theorem we can associate a continuous operator $\Lambda^{(+)}: \mathscr{D}(M, \mathscr{V}) \rightarrow \mathscr{D}^{\prime}(M, \mathscr{V})$ such that

$$
\Lambda(f, g)=-\int_{M} \beta\left(f, \Lambda^{(+)} g\right) d \Omega_{g}, \quad f, g \in \mathscr{D}(M, \mathscr{V}) .
$$

Furthermore, let us define $\Lambda^{(-)}:=\mathrm{i} S-\Lambda^{(+)}$in the fermionic case and $\Lambda^{(-)}:=-\mathrm{i} S+\Lambda^{(+)}$ in the bosonic case. The maps $\Lambda^{( \pm)}$will be called distributional two-point functions of $\omega$. Observe that since $\Lambda$ vanishes on $\operatorname{Ker} S$, it follows from (1) of Theorem 2.4.7 and formal self-adjointness of $D$ that $\Lambda^{( \pm)}$satisfy

$$
D \Lambda^{( \pm)}=\Lambda^{( \pm)} D=0 .
$$

Conversely, two continuous maps $\Lambda^{(+)}, \Lambda^{(-)}: \mathscr{D}(M, \mathscr{V}) \rightarrow \mathscr{D}^{\prime}(M, \mathscr{V})$ are distributional two-point functions of a quasi-free state on $\operatorname{CAR}(\mathscr{D}(M, \mathscr{V}) / \operatorname{Ker} S, Q)$ iff

$$
\begin{aligned}
(D) & D \Lambda^{( \pm)}=\Lambda^{( \pm)} D=0 \\
(P o s) & \beta \Lambda^{( \pm)} \geq 0 \\
(C A R) & \Lambda^{(+)}+\Lambda^{(-)}=\mathrm{i} S .
\end{aligned}
$$

The analogous condition for $\Lambda^{( \pm)}$to define a quasi-free state on $\operatorname{CCR}(\mathscr{D}(M, \mathscr{V}) / \operatorname{Ker} S, Q)$ reads

$$
\begin{aligned}
(D) & D \Lambda^{( \pm)}=\Lambda^{( \pm)} D=0 \\
(P o s) & \beta \Lambda^{( \pm)} \geq 0 \\
(C C R) & \Lambda^{(+)}-\Lambda^{(-)}=\mathrm{i} S .
\end{aligned}
$$

Note that since the causal propagator is a bi-solution for $D,(D)$ is equivalent to one of the conditions $D \Lambda^{(+)}=\Lambda^{(+)} D=0$ or $D \Lambda^{(-)}=\Lambda^{(-)} D=0$ (provided that $(C A R)$ or 
$(C C R)$ holds).

2.7.1. Multiplication by smooth sections. In applications it is often convenient to replace a given pre-normally hyperbolic operator $D$ by $a D b$, where $a, b \in$ $\mathscr{E}(M, \mathscr{L}(\mathscr{V}))$ are suitable chosen fiberwise invertible smooth sections. Although multiplying by both sides with smooth sections does break in general the property of being normally hyperbolic, it is easy to see that $a D b$ is pre-normally hyperbolic if and only if $D$ is pre-normally hyperbolic. It turns out that the charge forms corresponding to $D$ and $a D b$ are related in a simple way if $(a b)^{\dagger}=a b$.

Proposition 2.7.1. Let $a, b \in \mathscr{E}(M, \mathscr{L}(\mathscr{V}))$ be fiberwise invertible, let $(M, \mathscr{V}, D)$ be a field theory triple with hermitian structure $\beta$ and denote $Q$ the associated charge form. Assume that $a b$ is formally self-adjoint w.r.t. $\beta$ and set $D^{\prime}:=a D b$. Then $\left(M, \mathscr{V}, D^{\prime}\right)$ is a field theory triple with hermitian structure $\beta^{\prime}$ and charge form $Q^{\prime}$ given by

$$
\beta^{\prime}(f, g)=\beta\left(b^{\dagger} a^{-1} f, g\right), \quad Q^{\prime}(f, g)=Q\left(a^{-1} f, a^{-1} g\right), \quad f, g \in \mathscr{D}(M, \mathscr{V}) .
$$

Proof. We have to check that $D^{\prime}$ is formally self-adjoint w.r.t. $\beta^{\prime}$. Indeed, for all $f, g$ we have

$$
\begin{aligned}
\beta^{\prime}\left(D^{\prime} f, g\right) & =\beta\left(b^{\dagger} a^{-1} a D b f, g\right)=\beta\left(f, b^{\dagger} D b g\right) \\
& =\beta\left(b^{\dagger} a^{-1} f, b^{-1} a^{\dagger} b^{\dagger} D b g\right)=\beta\left(b^{\dagger} a^{-1} f, a D b g\right)=\beta^{\prime}\left(f, D^{\prime} g\right),
\end{aligned}
$$

where $(\cdot)^{\dagger}$ denotes the formal adjoint w.r.t. $\beta$. The causal propagator for $D^{\prime}$ equals $S^{\prime}=b^{-1} S a^{-1}$, hence

$$
Q^{\prime}=\mathrm{i} \beta^{\prime} S^{\prime}=\beta b^{\dagger} a^{-1} b^{-1} S a^{-1}=\beta\left(a^{-1}\right)^{\dagger} S a^{-1} .
$$

It follows that if $\Lambda(\cdot, \cdot)$ is the two-point function of a quasi-free state on the CAR or CCR $C^{*}$-algebra corresponding to the triple $(M, \mathscr{V}, D)$ then $\Lambda\left(a^{-1} \cdot a^{-1} \cdot\right)$ defines a quasi-free state for the triple $\left(M, \mathscr{V}, D^{\prime}\right)$. Note also that if $\Lambda^{( \pm)}$are distributional two-point functions associated to $\Lambda(\cdot, \cdot)$, then $\left(a^{-1}\right)^{\dagger} \Lambda^{( \pm)} a^{-1}$ are two-point functions associated to $\Lambda\left(a^{-1} \cdot a^{-1} \cdot\right)$. 


\section{CHAPTER 3}

\section{The Hadamard condition}

On Minkowski space, if no external potentials are present, there exists a distinguished state - the vacuum $\omega_{\text {vac }}$, which is uniquely defined by the translationinvariance property. It can also be characterized as the ground state w.r.t. time evolution and has the interpretation of a state of lowest energy.

On a generic globally hyperbolic space-time or in the presence of external potentials which vary in time, neither of these conditions make sense and the choice of a preferred state is problematic. On the other hand, the formulation of interacting Quantum Field Theory and semi-classical Einstein equations requires well-definiteness of the evaluation of a given state $\omega$ on Wick powers : $\phi(x):^{k},: \psi^{k}(x) \psi^{* l}(x)$ : of noninteracting fields. The best-to-date way to define $\mapsto \omega\left(: \psi(x) \psi^{*}(x):\right)$ as a distribution on $M$ consists of viewing $\omega\left(: \psi(x) \psi^{*}(y):\right)$ as a distribution on $M \times M$ in the variables $x$ and $y$, substracting its singular part and then taking the limit of conciding points $x \rightarrow y$. The discovery that this can be done consistently for a class of states which possess the same $x \rightarrow y$ behaviour as the Hadamard parametrix led to the formulation of the Hadamard condition [Wal77].

A parallel line of investigation was initiated by Duistermaat and Hörmander in their works on Fourier integral operators [Hör71, DH72]. Using methods of microlocal analysis they constructed parametrices distinguished modulo smooth terms by their wave front set and proved propagation of singularity theorems valid for a class of differential operators which included normally hyperbolic ones. The relation between the microlocal approach and the constructions developed by field theorists remained however obscure for years ${ }^{1}$.

In his seminal paper [Rad96], Radzikowski was able to make the precise connection between the Hadamard parametrix and the theory of distinguished parametrices of Duistermaat and Hörmander and he proved that the Hadamard condition can be rephrased as a condition on the wave front set of the two-point function. This initiated a series of important results, including most importantly a proof of perturbative renormalizability of field theory on curved space-time by Brunetti and Fredenhagen [BF00]. The condition obtained by Radzikowski for the Klein-Gordon field was generalized to the case of Dirac fields in [SV01, Hol01] and consequences in the case of non-vanishing external potentials were investigated in [Mar03].

The aim of this chapter is to introduce the reader to the microlocal formulation of the Hadamard condition and derive the connected results in a unified way for both

${ }^{1}$ See [Wal12a] for a historical overview on the developments in quantum field theory on curved spacetime. 
bosonic and fermionic fields. In contrast to what is done in most of the literature we do not assume invariance of the states under a charge conjugation, motivated by the fact that no charge conjugation exists if non-vanishing external potentials are present. Our results are to a large extent a formal generalization of the work of Hollands [Hol01] (where only the Dirac case is considered), the proofs we give are also somewhat simplified and thus of didactical value.

\subsection{Parametrices}

In the study of singularities of solutions of elliptic and hyperbolic partial differential equations, it is often useful to replace actual fundamental solutions, usually difficult to construct explicitely, by parametrices, which can be thought as 'fundamental solutions up to smooth remainders

Definition 3.1.1. Let $P: \mathscr{D}(M, \mathscr{V}) \rightarrow \mathscr{D}(M, \mathscr{W})$ be a differential operator. We say that $S: \mathscr{D}(M, \mathscr{W}) \rightarrow \mathscr{D}^{\prime}(M, \mathscr{V})$ is a parametrix for $P$ if both $P S$ and $S P$ differ from the identity on by an operator with smooth Schwartz kernel.

We will write $A=B \bmod C^{\infty}$ if $A$ and $B$ differ from the identity by an operator with smooth Schwartz kernel. In this notation $S$ is a parametrix of $P$ if

$$
P S=\operatorname{id}_{\mathscr{D}(M, \mathscr{W})} \bmod C^{\infty}, \quad S P=\operatorname{id}_{\mathscr{D}(M, \mathscr{V})} \bmod C^{\infty} .
$$

\subsection{Wave front set}

One of the classical results in Fourier analysis - the Paley-Wiener-Schwartz theorem, entails that a compactly supported distribution $v \in \mathscr{E}^{\prime}\left(\mathbb{R}^{n}\right)$ is smooth if its Fourier transform satisfies an estimate of the form

$$
|\widehat{v}(\xi)| \leq C_{N}\langle\xi\rangle^{-2 N}, \quad N \in \mathbb{N}_{0},
$$

where $\langle\xi\rangle=(1+|\xi|)^{1 / 2}$. In order to obtain a precise description of the singularities of a generic distribution $u \in \mathscr{D}^{\prime}\left(\mathbb{R}^{n}\right)$, we localise it around a given point $x_{0} \in \mathbb{R}^{n}$ by multiplying it with a test function $\varphi \in \mathscr{D}\left(\mathbb{R}^{n}\right)$ with arbitrarily small support and such that $\varphi\left(x_{0}\right) \neq 0$, obtaining this way a compactly supported distribution $\varphi u$. If $u$ has a singularity at $x_{0}$, this means that $\varphi u$ is not smooth and we can ask what are the directions $\xi$ responsible for the violation of inequality (3.2.1). This idea is at the heart of the definition of the wave front set of a distribution $u \in \mathscr{D}^{\prime}\left(\mathbb{R}^{n}\right)$, which is a subset of $\mathbb{R}^{n} \times \dot{\mathbb{R}}^{n}\left(\dot{\mathbb{R}}^{n}:=\mathbb{R}^{n} \backslash\{0\}\right)$ denoted $\mathrm{WF}(u)$ and defined as follows.

Definition 3.2.1. A point $\left(x_{0}, \xi_{0}\right) \in \mathbb{R}^{n} \times \dot{\mathbb{R}}^{n}$ does not belong to $\mathrm{WF}(u)$ iff there exists $\varphi \in \mathscr{D}\left(\mathbb{R}^{n}\right)$, with $\varphi\left(x_{0}\right) \neq 0$, and an open cone $V_{x_{0}} \in \dot{\mathbb{R}}^{n}$ (i.e., $t V_{x_{0}} \subset V_{x_{0}}$ for all $t>0)$ such that $\xi_{0} \in V_{x_{0}}$ and

$$
|\widehat{\varphi u}(\xi)| \leq C_{N}\langle\xi\rangle^{-2 N}, \quad N \in \mathbb{N}_{0}
$$

for all $\xi \in V_{x_{0}}$. 
If $\pi: \mathbb{R}^{n} \times \dot{\mathbb{R}}^{n} \rightarrow \mathbb{R}^{n}$ is the projection on the first component, then it can be checked that the set $\pi \mathrm{WF}(u)$ is nothing but the singular support of $u$. Consequently, $u \in \mathscr{D}^{\prime}\left(\mathbb{R}^{n}\right)$ is smooth if and only if its wave front set is empty.

To extend the definition of the wave front set to distributions on a smooth manifold $M$, let us first discuss how $\mathrm{WF}(u)$ transforms under diffeomorphism. Let $\Phi: U \rightarrow V$ be a diffeomorphism between open subsets of $\mathbb{R}^{n}$ and let $u \in \mathscr{D}^{\prime}(U)$. The pushforward of $u$ by $\Phi$ is the distribution $\Phi_{*} u \in \mathscr{D}^{\prime}(V)$ defined by $\left\langle\Phi_{*} u, \varphi\right\rangle=\langle u, \varphi \circ \Phi\rangle$ for $\varphi \in \mathscr{D}(V)$. It holds that

$$
\mathrm{WF}\left(\Phi_{*} u\right)=\left\{(\Phi(x), \xi): x \in U,\left(x,\left(\Phi_{x}^{\prime}\right)^{\mathrm{t}} \xi\right) \in \mathrm{WF}(u)\right\},
$$

where $\left(\Phi_{x}^{\prime}\right)^{\mathrm{t}}$ is the transpose of the Jacobi matrix $\left(\Phi_{x}^{\prime}\right)^{\mathrm{t}}$ of $\Phi$.

In particular, it follows that the $\xi$ component of $\mathrm{WF}(u)$ behaves like a covector under the action of diffeomorphisms. Therefore, we can identify $\mathrm{WF}(u)$ with a subset of $T^{*} U \backslash \mathcal{Z}$, i.e. the cotangent bundle of $U$ with the zero section $\mathcal{Z} \in T^{*} U$ removed.

If now $u \in \mathscr{D}^{\prime}(M)$ is a distribution on a manifold and $\left(U_{\alpha}, \Phi_{\alpha}\right)$ is a coordinate system of $M$, the push-forward of $\left.u\right|_{U_{\alpha}}$ by the diffeomorphism $\Phi_{\alpha}$ is a distribution on an open neighbourhood of the origin in $\mathbb{R}^{n}$. The relation (3.2.2) can be thus used to define $\operatorname{WF}\left(\left.u\right|_{U_{\alpha}}\right)$ as a subset of $T^{*} U_{\alpha} \backslash \mathcal{Z}$. The wave front set of $u$ is then defined by

$$
\mathrm{WF}(u):=\bigcup_{\alpha} \mathrm{WF}\left(\left.u\right|_{U_{\alpha}}\right) \subset T^{*} M \backslash \mathcal{Z} .
$$

Again, it is not difficult to show that $u \in \mathscr{D}^{\prime}(M)$ is a smooth density if and only if $\mathrm{WF}(u)$ is empty.

The definition of the wave front set extends easily to elements of $\mathscr{D}^{\prime}(M, \mathscr{V})$, where $\mathscr{V}$ is vector bundle of rank $k<\infty$. Namely, if $u \in \mathscr{D}^{\prime}(M, \mathscr{V})$ and $u_{i, \alpha}(i=1, \ldots, k)$ are the components of $u$ in a local trivialization of $\mathscr{V}$ relative to a covering $\left\{U_{\alpha}\right\}$ of $M$, we set

$$
\mathrm{WF}\left(\left.u\right|_{U_{\alpha}}\right):=\bigcup_{i} \mathrm{WF}\left(u_{i, \alpha}\right) \subset T^{*} U_{\alpha} \backslash \mathcal{Z}
$$

and then define as $\mathrm{WF}(u)$ as in (3.2.3). It is not difficult to prove that this definition does not depend on the choice of the trivialization.

3.2.1. Operations on distributions. The practical relevance of the wave front set in applications in Quantum Field Theory is based on the fact that it can be used to express sufficient conditions for the well-definiteness of operations on distributions which are in general ill-defined, such as restriction to submanifolds or pointwise products. In this section we briefly recall well-known properties of the wave front set with respect to basic operations on distributions.

It is convenient to introduce first the following definitions. A set $\Gamma \subset T^{*} M \backslash \mathcal{Z}$ is said to be conic if

$$
(x, \xi) \in \Gamma \Rightarrow(x, t \xi) \in \Gamma, \quad t \in \mathbb{R} .
$$

In particular, the wave front set of a distribution is always a conic set. If $\Gamma \subset T^{*} M \backslash \mathcal{Z}$ is conic, we define

$$
-\Gamma:=\{(x,-\xi):(x, \xi) \in \Gamma\} \subset T^{*} M \backslash \mathcal{Z} .
$$


Let $M_{1}, M_{2}$ be two manifolds, let $\mathcal{Z}_{1}, \mathcal{Z}_{2}, \mathcal{Z}$ be the zero section of respectively $T^{*} M_{1}$, $T^{*} M_{2}, T^{*}\left(M_{1} \times M_{2}\right)$, and let $\Gamma \subset T^{*}\left(M_{1} \times M_{2}\right) \backslash \mathcal{Z}$ be a conic set. The elements of $T^{*}\left(M_{1} \times M_{2}\right) \backslash \mathcal{Z}$ will be denoted by $\left(x_{1}, \xi_{1}, x_{2}, \xi_{2}\right)$. We set

$$
\begin{aligned}
& \Gamma^{\prime}:=\left\{\left(x_{1}, \xi_{1}, x_{2},-\xi_{2}\right):\left(x_{1}, \xi_{1}, x_{2}, \xi_{2}\right) \in \Gamma\right\} \subset T^{*}\left(M_{1} \times M_{2}\right) \backslash \mathcal{Z}, \\
& \operatorname{Exch}(\Gamma):=\left\{\left(x_{2}, \xi_{2}, x_{1}, \xi_{1}\right):\left(x_{1}, \xi_{1}, x_{2}, \xi_{2}\right) \in \Gamma\right\} \subset T^{*}\left(M_{2} \times M_{1}\right) \backslash \mathcal{Z}, \\
& { }_{M_{1}} \Gamma:=\left\{\left(x_{1}, \xi_{1}\right): \exists x_{2} \text { such that }\left(x_{1}, \xi_{1}, x_{2}, 0\right) \in \Gamma\right\} \subset T^{*} M_{1} \backslash \mathcal{Z}_{1}, \\
& \Gamma_{M_{2}}:=\left\{\left(x_{2}, \xi_{2}\right): \exists x_{1} \text { such that }\left(x_{1}, 0, x_{2}, \xi_{2}\right) \in \Gamma\right\} \subset T^{*} M_{2} \backslash \mathcal{Z}_{2} .
\end{aligned}
$$

If $A: \mathscr{D}(M, \mathscr{V}) \rightarrow \mathscr{D}^{\prime}(M, \mathscr{V})$ is a continuous operator with Schwartz kernel $K_{A}$, we will write for simplicity $\mathrm{WF}(A):=\mathrm{WF}\left(K_{A}\right)$ and $\mathrm{WF}^{\prime}(A):=\mathrm{WF}\left(K_{A}\right)^{\prime}$. We will see that in many situations it is more natural to use $\mathrm{WF}^{\prime}$ instead of WF.

We refer the reader to [Hör83] for the proof of the theorem below and the construction of distributions such as $\left.u\right|_{Y}$.

TheOREM 3.2.2. The wave front set has the following properties:

(1) Sum: if $u, v \in \mathscr{D}^{\prime}(M, \mathscr{V})$ then $\mathrm{WF}(u+v) \subset \mathrm{WF}(u) \cup \mathrm{WF}(v)$.

(2) Complex conjugation: if $u \in \mathscr{D}^{\prime}(M, \mathscr{V})$ then $\mathrm{WF}(\bar{u})=-\mathrm{WF}(u)$.

(3) Action of a PDO: let $P: \mathscr{D}(M, \mathscr{V}) \rightarrow \mathscr{D}(M, \mathscr{V})$ be a partial differential operator and denote $\sigma_{P}(x, \xi)$ its principal symbol. Then

$$
\mathrm{WF}(P u) \subset \mathrm{WF}(u) \subset \mathscr{N} \cup \mathrm{WF}(P u), \quad u \in \mathscr{D}^{\prime}(M, \mathscr{V}),
$$

where $\mathscr{N}$ is the characteristic submanifold of $P$, defined by

$$
\mathscr{N}=\left\{(x, \xi) \in T^{*} M \backslash \mathcal{Z}: \operatorname{det} \sigma_{P}(x, \xi)=0\right\} .
$$

(4) Tensor product: if $u \in \mathscr{D}^{\prime}\left(M_{1}\right), v \in \mathscr{D}^{\prime}\left(M_{2}\right)$ then

$$
\begin{aligned}
\mathrm{WF}(u \otimes v) \subset(\mathrm{WF}(u) \times \mathrm{WF}(v)) & \cup((\operatorname{supp} u \times\{0\}) \times \mathrm{WF}(v)) \\
& \cup(\mathrm{WF}(u) \times(\operatorname{supp} v \times\{0\}) .
\end{aligned}
$$

(5) Restriction to a sub-manifold: Let $Y \subset M$ be a sub-manifold. The co-normal bundle to $Y$ in $M$ is by definition the bundle

$$
T_{Y}^{*} M:=\left\{(y, \xi) \in T^{*} M \backslash \mathcal{Z}: y \in Y, \xi \cdot v=0 \forall v \in T_{y} Y\right\} .
$$

If $u \in \mathscr{D}^{\prime}(M, \mathscr{V})$ and $\operatorname{WF}(u) \cap T_{Y}^{*} M=\emptyset$ then the restriction $u_{\mid Y}$ of $u$ to $Y$ is well defined and

$$
\mathrm{WF}\left(u_{\mid Y}\right) \subset\left\{\left(y, \xi_{\mid T_{y} Y}\right): y \in Y,(y, \xi) \in \mathrm{WF}(u)\right\} .
$$

(6) Kernels: let $A: \mathscr{D}\left(M_{2}\right) \rightarrow \mathscr{D}^{\prime}\left(M_{1}\right)$ be linear continuous. Then $A u$ is well defined for $u \in \mathscr{E}^{\prime}\left(M_{2}\right)$ if $\mathrm{WF}(u) \cap \mathrm{WF}^{\prime}(A)_{M_{2}}=\emptyset$ and in such case

$$
\mathrm{WF}(A u) \subset{ }_{M_{1}} \mathrm{WF}(A) \cup \mathrm{WF}^{\prime}(A) \circ \mathrm{WF}(u) .
$$

(7) Composition: Let $A_{1} \in \mathscr{D}\left(M_{1}\right) \rightarrow \mathscr{D}^{\prime}\left(M_{2}\right), A_{2} \in \mathscr{D}\left(M_{1}\right) \rightarrow \mathscr{D}^{\prime}\left(M_{2}\right)$ be continuous and assume that $K_{2}$ is properly supported. Then $A_{1} \circ A_{2}$ is well defined if

$$
\mathrm{WF}^{\prime}\left(A_{1}\right)_{M_{2}} \cap{ }_{M_{2}} \mathrm{WF}^{\prime}\left(A_{2}\right)=\emptyset \text {, }
$$


and in such case $\mathrm{WF}^{\prime}\left(A_{1} \circ A_{2}\right)$ is contained in

$$
\left(\mathrm{WF}^{\prime}\left(A_{1}\right) \circ \mathrm{WF}^{\prime}\left(A_{2}\right)\right) \cup\left({ }_{M_{1}} \mathrm{WF}^{\prime}\left(A_{1}\right) \times \mathcal{Z}_{3}\right) \cup\left(\mathcal{Z}_{1} \times \mathrm{WF}^{\prime}\left(A_{2}\right)_{M_{3}}\right)
$$

Property (5) can be used to define products of distributions. To see that, observe that if $u$ and $v$ are distributions on $M$ and one of them is smooth, their product can be obtained by restricting the tensor product $u \otimes v$ to the diagonal $\Delta_{M}=M \times M$. By (5), since the co-normal bundle of $\Delta_{N}$ is

$$
T_{\Delta_{M}}^{*} M=\left\{(x, \xi, x,-\xi) \in T^{*}(M \times M) \backslash \mathcal{Z}:(x, \xi) \in T^{*} M \backslash \mathcal{Z}\right\},
$$

the distribution $u v:=(u \otimes v)_{\mid \Delta_{M}}$ is still well-defined if

$$
\mathrm{WF}(u) \cap-\mathrm{WF}(v)=\emptyset,
$$

and in such case it satisfies

$$
\mathrm{WF}(u v) \subset\left\{(x, \xi+\eta) \in T^{*} M \backslash \mathcal{Z}:(x, \xi) \in \mathrm{WF}(u) \cup \mathcal{Z},(x, \eta) \in \mathrm{WF}(v) \cup \mathcal{Z}\right\} .
$$

Condition (3.2.4) also allows to define an operation that generalizes the pairing $\langle\cdot, \cdot\rangle$ between compactly supported distributions $\mathscr{E} \prime$ and $\mathscr{E}$. Indeed, if $u$ is compactly supported and (3.2.4) holds, then $\langle u, v\rangle:=\langle u v, 1\rangle$ is well-defined.

\subsection{Distinguished parametrices}

In what follows, we review the theory of distinguished parametrices of Duistermaat and Hörmander [DH72].

Let $P: \mathscr{E}(M, \mathscr{V}) \rightarrow \mathscr{E}(M, \mathscr{V})$ be a differential operator and let us denote $\mathscr{N}$ its characteristic manifold, i.e.

$$
\mathscr{N}=\left\{(x, \xi) \in T^{*} M \backslash \mathcal{Z}: \operatorname{det} \sigma_{P}(x, \xi)=0\right\} .
$$

A bicharacteristic of $P$ is by definition a curve in $\mathscr{N}$ generated by the Hamiltonian flow with Hamiltonian $\sigma_{P}$. In other words, this means that a bicharacteristic is an integral curve in $\mathscr{N}$ of the system of equations

$$
\frac{d x_{i}}{d \tau}=\frac{\partial \sigma_{P}(x(\tau), \xi(\tau))}{\partial \xi_{i}}, \quad \frac{d \xi_{i}}{d \tau}=-\frac{\partial \sigma_{P}(x(\tau), \xi(\tau))}{\partial x_{i}}, \quad i=0, \ldots, d
$$

In what follows we will use the notation $X=(x, \xi) \in T^{*} M \backslash \mathcal{Z}$. The bi-characteristic passing through $X_{0}=\left(x_{0}, \xi_{0}\right) \in T^{*} M \backslash \mathcal{Z}$ will be denoted $B\left(X_{0}\right)$.

Definition 3.3.1. A differential operator $P: \mathscr{E}(M, \mathscr{V}) \rightarrow \mathscr{E}(M, \mathscr{V})$ is said to be of real principal type if its principal symbol $\sigma_{P}$ is real and homogeneous and there exists no complete bicharacteristic of $P$ whose projection onto $M$ is included in a compact subset of $M$.

Let us define an equivalence relation between elements of $\mathscr{N}$ by

$$
X_{1} \sim X_{2} \Leftrightarrow X_{2} \in B\left(X_{1}\right) .
$$

We write $X_{1} \succ X_{2}$ (resp. $X_{1} \prec X_{2}$ ) if $X_{1} \sim X_{2}$ and $X_{1}$ comes strictly after (resp. before) $X_{2}$ wrt. the natural parameter on the bicharacteristic curve through $X_{1}$ and $X_{2}$. 
Hörmander's propagation of singularities theorem states:

TheOrem 3.3.2. Let $P$ be a differential operator of real principal type and suppose $u \in \mathscr{D}^{\prime}(M)$ satisfies $P u \in \mathscr{E}(M)$. Then $\mathrm{WF}(u) \subset \mathscr{N}$ and

$$
X \in \mathrm{WF}(u) \Rightarrow B(X) \subset \mathrm{WF}(u) .
$$

In what follows we will need an additional assumption on $P$. Namely, for an arbitrary compact set $K$, let us consider the set of all bicharacteritics $P$ whose both endpoints are in $K$. If there exists another compact set $K^{\prime}$ which contains all these curves, one says that $M$ is pseudo-convex w.r.t. $P$.

The bicharacteristic relation of $P$ is the set

$$
\mathscr{C}:=\left\{\left(X_{1}, X_{2}\right) \in \mathscr{N} \times \mathscr{N}: X_{1} \sim X_{2}\right\} .
$$

Denote $\Delta_{\mathscr{N}}$ the diagonal in $\mathscr{N} \times \mathscr{N}$, i.e.

$$
\Delta_{\mathscr{N}}:=\{(X, X): X \in \mathscr{N}\} .
$$

Observe that $\mathscr{C} \backslash \Delta_{\mathscr{N}}$ is the disjoint union of two open sets, namely

$$
\mathscr{C}^{+}:=\left\{\left(X_{1}, X_{1}\right) \in \mathscr{C}: X_{1} \succ X_{1}\right\}, \quad \mathscr{C}^{-}:=\left\{\left(X_{1}, X_{2}\right) \in \mathscr{C}: X_{1} \prec X_{2}\right\},
$$

which satisfy $\operatorname{Exch}\left(\mathscr{C}^{+}\right)=\mathscr{C}^{-}$.

DeFinition 3.3.3. An orientation of $\mathscr{C}$ is a partition $\mathscr{C} \backslash \Delta_{\mathscr{N}}=\mathscr{C}^{1} \cup \mathscr{C}^{2}$ into two disjoint open subsets $\mathscr{C}^{1}, \mathscr{C}^{2} \subset \mathscr{C} \backslash \Delta_{\mathscr{N}}$ s.t. $\operatorname{Exch}\left(\mathscr{C}^{1}\right)=\mathscr{C}^{2}$.

Observe that if $\left(\mathscr{C}^{1}, \mathscr{C}^{2}\right)$ is an orientation of $\mathscr{C}$ then the property $\operatorname{Exch}\left(\mathscr{C}^{1}\right)=\mathscr{C}^{2}$ entails that none of the two sets $\mathscr{C}^{1}, \mathscr{C}^{2}$ can be empty. It should also be noted that $\mathscr{C}^{1}$ must be the union of connected components of $\mathscr{C} \backslash \Delta_{\mathscr{N}}$ and the same is true for $\mathscr{C}^{2}$.

All orientations of $\mathscr{C}$ can be obtained in the following way from connected components of $\mathscr{N}$. Let us set

$$
\mathscr{C}^{ \pm}(X):=\mathscr{C}^{ \pm} \cap B(X) \times B(X) .
$$

Obviously, $\operatorname{Exch}\left(\mathscr{C}^{+}(X)\right)=\mathscr{C}^{-}(X)$. Then the following result holds true.

Proposition 3.3.4. Let $\mathscr{C}$ be a bi-characteristic relation.

(1) Let $\mathscr{C} \backslash \Delta_{\mathscr{N}}=\mathscr{C}^{1} \cup \mathscr{C}^{2}$ be an orientation of $\mathscr{C}$. Set

$$
\mathscr{N}^{i}:=\left\{X \in \mathscr{N}: \mathscr{C}^{+}(X) \subset \mathscr{C}^{i}\right\}, \quad i=1,2 .
$$

Then $\mathscr{N}^{i}$ are unions of connected components of $\mathscr{N}$.

(2) Conversely let $\mathscr{N}=\mathscr{N}^{1} \cup \mathscr{N}^{2}$ be a partition of $\mathscr{N}$ into two open and closed subsets of $\mathscr{N}$. Set

$$
\begin{aligned}
\mathscr{C}^{1} & :=\bigcup_{X \in \mathscr{N}^{1}} \mathscr{C}^{+}(X) \cup \bigcup_{X \in \mathscr{N}^{2}} \mathscr{C}^{-}(X), \\
\mathscr{C}^{2} & :=\bigcup_{X \in \mathscr{N}^{2}} \mathscr{C}^{+}(X) \cup \bigcup_{X \in \mathscr{N}^{1}} \mathscr{C}^{-}(X) .
\end{aligned}
$$

Then $\left(\mathscr{C}^{1}, \mathscr{C}^{2}\right)$ is an orientation of $\mathscr{C}$. 
Recall that an operator $E$ is a parametrix of $P$ if $P E=E P=\operatorname{id} \bmod C^{\infty}$. We are ready to formulate the main result of Duistermaat and Hörmander's theory:

TheOREM 3.3.5 ([DH72]). Let $P: \mathscr{E}(M, \mathscr{V}) \rightarrow \mathscr{E}(M, \mathscr{V})$ be a differential operator of real principal type such that $M$ is pseudo-convex w.r.t. $P$ and let $\left(\mathscr{C}^{1}, \mathscr{C}^{2}\right)$ be an orientation of $\mathscr{C}$. Then there exist parametrices $E^{i}$ of $P, i=1,2$, such that

$$
\mathrm{WF}^{\prime}\left(E^{i}\right) \subset \Delta^{*} \cup \mathscr{C}^{i}
$$

where $\Delta^{*}$ is the diagonal in $T^{*} M \backslash \mathcal{Z} \times T^{*} M \backslash \mathcal{Z}$. Any left or right parametrix which satisfies (3.3.3) is equal to $E^{i}$ modulo $C^{\infty}$.

3.3.1. Distinguished parametrices of the Klein-Gordon operator. We now apply the theory of distinguished parametrices of Duistermaat and Hörmander to the special case of a normally hyperbolic operator $P$ on a globally hyperbolic spacetime $(M, g)$. It is proved in [Rad96] that in such case $P$ is of real principal type and $M$ is pseudo-convex for $P$.

Recall that $V_{x \pm} \subset T_{x} M$ are the open future/past light cones and the dual cones $V_{x \pm}^{*}$ are subsets of $T_{x}^{*} M$ defined by

$$
V_{x \pm}^{*}:=\left\{\xi \in T_{x}^{*} M: \xi \cdot v>0, \forall v \in V_{x \pm}, v \neq 0\right\} .
$$

The characteristic manifold of $P$ is given by

$$
\mathscr{N}=\left\{(x, \xi) \in T^{*} M \backslash \mathcal{Z}: g^{\mu \nu}(x) \xi_{\mu} \xi_{\nu}=0\right\} .
$$

Recall that the bicharacheristic relation of $P$ is the set

$$
\mathscr{C}=\left\{\left(X_{1}, X_{2}\right) \in \mathscr{N} \times \mathscr{N}: X_{1} \sim X_{2}\right\} .
$$

It can be easily shown that in our case of interest, $X_{1} \sim X_{2}$ iff $X_{1}=\left(x_{1}, \xi_{1}\right)$ and $X_{2}=$ $\left(x_{2}, \xi_{2}\right)$ are on the same null geodesic strip, that is if there is a null geodesic through $x_{1}$ and $x_{2}$ with tangent vector $\xi_{1}$ ending at $x_{2}$ with tangent vector $\xi_{2}$. Moreover,

$$
\begin{aligned}
& X_{1} \succ X_{2} \Longleftrightarrow X_{1} \sim X_{2}, \quad x_{1} \in J^{ \pm}\left(x_{2}\right) \text { if } \xi_{1} \in V_{x_{1} \pm}^{*}, \\
& X_{1} \prec X_{2} \Longleftrightarrow X_{1} \sim X_{2}, \quad x_{1} \in J^{\mp}\left(x_{2}\right) \text { if } \xi_{1} \in V_{x_{1} \pm}^{*} .
\end{aligned}
$$

Observe that $\mathscr{N}$ has two connected components invariant under the bicharacteristic flow, namely

$$
\mathscr{N}_{ \pm}:=\left\{X \in \mathscr{N}: \xi \in V_{x \pm}^{*}\right\}
$$

This gives 4 partitions of $\mathscr{N}$ into disjoint open sets, and hence 4 orientations of $\mathscr{C}$ and 4 distinguished parametrices. They have the following traditional names:

Feynman parametrix: take the partition $\mathscr{N}=\mathscr{N} \cup \emptyset$, this corresponds to the orientation $\left(\mathscr{C}_{\mathrm{F}}^{1}, \mathscr{C}_{\mathrm{F}}^{2}\right)$, where

$$
\mathscr{C}_{\mathrm{F}}^{1}=\mathscr{C}^{+}=\left\{\left(X_{1}, X_{2}\right) \in \mathscr{C}: X_{1} \succ X_{2}\right\}, \quad \mathscr{C}_{\mathrm{F}}^{2}=\mathscr{C}^{-}=\left\{\left(X_{1}, X_{2}\right) \in \mathscr{C}: X_{1} \prec X_{2}\right\} .
$$

The parametrix $E_{\mathrm{F}}:=E_{\mathrm{F}}^{1}=E_{\overline{\mathrm{F}}}^{2}$ is called the Feynman parametrix.

Anti-Feynman parametrix: the partition $\mathscr{N}=\emptyset \cup \mathscr{N}$ corresponds to the orientation $\left(\mathscr{C}_{\overline{\mathrm{F}}}, \mathscr{C}_{\overline{\mathrm{F}}}^{2}\right)$, where

$$
\mathscr{C}_{\overline{\mathrm{F}}}^{1}=\mathscr{C}^{-}, \quad \mathscr{C}_{\overline{\mathrm{F}}}^{2}=\mathscr{C}^{+} .
$$


The parametrix $E_{\overline{\mathrm{F}}}:=E_{\overline{\mathrm{F}}}^{1}=E_{\mathrm{F}}^{2}$ is called the anti-Feynman parametrix.

Retarded parametrix: take $\mathscr{N}=\mathscr{N}_{+} \cup \mathscr{N}_{-}$, this corresponds to the orientation $\left(\mathscr{C}_{-}^{1}, \mathscr{C}_{-}^{2}\right)$, where

$$
\mathscr{C}_{-}^{1}=\left\{\left(X_{1}, X_{2}\right) \in \mathscr{C}: x_{1} \in J^{+}\left(x_{2}\right)\right\}, \quad \mathscr{C}_{-}^{2}=\left\{\left(X_{1}, X_{2}\right) \in \mathscr{C}: x_{1} \in J^{-}\left(x_{2}\right)\right\} .
$$

The parametrix $E_{\mathrm{R}}:=E_{\mathrm{R}}^{1}=E_{\mathrm{A}}^{2}$ is called the retarded parametrix.

Advanced parametrix: take $\mathscr{N}=\mathscr{N}_{-} \cup \mathscr{N}_{+}$, this corresponds to the orientation $\left(\mathscr{C}_{\mathrm{A}}^{1}, \mathscr{C}_{\mathrm{A}}^{2}\right)$, where

$$
\mathscr{C}_{\mathrm{A}}^{1}=\mathscr{C}_{-}^{2}, \quad \mathscr{C}_{\mathrm{A}}^{2}=\mathscr{C}_{-}^{1} .
$$

The parametrix $E_{\mathrm{A}}:=E_{\mathrm{A}}^{1}=E_{\mathrm{R}}^{2}$ is called the advanced parametrix.

The terminology above is motivated by the following important fact.

Proposition 3.3.6. Let $E_{\mathrm{A} / \mathrm{R}}$ be a retarded/advanced parametrix for $P$, and let $E_{+/-}$be the retarded/advanced fundamental solution for $P$ (cf. Section 2.4). Then

$$
E_{\mathrm{A}}=E_{+} \bmod C^{\infty}, \quad E_{\mathrm{R}}=E_{-} \bmod C^{\infty} .
$$

In the context of quantization and two-point functions, one is more interested in solutions rather than in fundamental solutions. The next lemma characterizes the wave front sets of differences of the distinguished parametrices, which are solutions of $P$ up to smooth remainders. The proof essentially follows [Jun95].

If $U \subset M$, and $\Gamma_{1}, \Gamma_{2} \in T^{*} M \backslash \mathcal{Z}$ are conic sets, we will say that $\Gamma_{1}=\Gamma_{2}$ above $U$ if $\Gamma_{1} \cap T^{*} U \backslash \mathcal{Z}=\Gamma_{2} \cap T^{*} U \backslash \mathcal{Z}$.

LEMMA 3.3.7. We have:

$$
\begin{aligned}
& \mathrm{WF}^{\prime}\left(E_{\mathrm{R}}-E_{\mathrm{A}}\right)=\mathscr{C}, \\
& \text { (2) } \mathrm{WF}^{\prime}\left(E_{\mathrm{R}}-E_{\mathrm{F}}\right)=\mathscr{C} \cap \mathscr{N}_{-} \times \mathscr{N}_{-}, \\
& \text {(3) } \mathrm{WF}^{\prime}\left(E_{\mathrm{A}}-E_{\mathrm{F}}\right)=\mathscr{C} \cap \mathscr{N}_{+} \times \mathscr{N}_{+} .
\end{aligned}
$$

Proof. (1): Since $E_{\mathrm{R}}, E_{\mathrm{A}}$ have disjoint wave front sets above $\left\{x_{1} \neq x_{2}\right\}$, it follows that above $\left\{x_{1} \neq x_{2}\right\}$

$$
\mathrm{WF}^{\prime}\left(E_{\mathrm{R}}-E_{\mathrm{A}}\right)=\mathrm{WF}^{\prime}\left(E_{\mathrm{R}}\right) \cup \mathrm{WF}^{\prime}\left(E_{\mathrm{A}}\right)=\mathscr{C} \backslash \Delta_{\mathscr{N}} .
$$

Since $P\left(E_{\mathrm{R}}-E_{\mathrm{A}}\right)=\left(E_{\mathrm{R}}-E_{\mathrm{A}}\right) P=0 \bmod C^{\infty}$, by the propagation of singularities theorem we obtain that $\Delta_{\mathscr{N}} \subset \mathrm{WF}^{\prime}\left(E_{\mathrm{R}}-E_{\mathrm{A}}\right)$. This proves (1).

(2): Above $\left\{\left(x_{1}, x_{2}\right): x_{1} \in J^{-}\left(x_{2}\right)\right\}$, we have

$$
\mathrm{WF}^{\prime}\left(E_{\mathrm{R}}-E_{\mathrm{F}}\right)=\mathrm{WF}^{\prime}\left(E_{\mathrm{F}}\right)=\left\{\left(X_{1}, X_{2}\right): x_{1} \in J^{-}\left(x_{2}\right), \xi \in V_{x_{1}+}^{*}\right\} .
$$

Using the propagation of singularities theorem for $E_{\mathrm{R}}-E_{\mathrm{F}}$ we obtain that $\mathrm{WF}^{\prime}\left(E_{\mathrm{R}}-\right.$ $\left.E_{\mathrm{F}}\right)=\mathscr{C} \cap \mathscr{N}_{-} \times \mathscr{N}_{-}$. The proof of (3) is analogous.

It follows directly from (1) and Proposition 3.3.6 that if $E$ is the causal propagator for $P$ then

$$
\mathrm{WF}(E)=\mathscr{C}=\left\{\left(X_{1}, X_{2}\right) \in \mathscr{N} \times \mathscr{N}: X_{1} \sim X_{2}\right\}
$$


3.3.2. Parametrices for prenormally hyperbolic operators. Consider now a pre-normally hyperbolic operator $D: \mathscr{E}(M, \mathscr{V}) \rightarrow \mathscr{E}(M, \mathscr{V})$. By definition, there exists a differential operator $\widetilde{D}$ such that $P:=D \widetilde{D}$ is normally hyperbolic. We have thus distinguished parametrices $E_{\mathrm{F}}, E_{\overline{\mathrm{F}}}$ and by setting

$$
S_{\mathrm{F}}:=\widetilde{D} E_{\mathrm{F}}, \quad S_{\overline{\mathrm{F}}}:=\widetilde{D} E_{\overline{\mathrm{F}}}
$$

we obtain parametrices for $D$. Recall also that if $E_{\mathrm{R}}, E_{\mathrm{A}}$ are the retarded and advanced propagator for $P$ then $S_{\mathrm{R}}:=\widetilde{D} E_{\mathrm{R}}$ and $S_{\mathrm{A}}:=\widetilde{D} E_{\mathrm{A}}$ are retarded and advanced propagators for $D$. As far as the wave front sets are concerned, in general we only have the relations

$$
\mathrm{WF}\left(S_{\mathrm{F}}\right) \subset \mathrm{WF}\left(E_{\mathrm{F}}\right), \quad \mathrm{WF}\left(S_{\overline{\mathrm{F}}}\right) \subset \mathrm{WF}\left(E_{\overline{\mathrm{F}}}\right) .
$$

and analogous ones for $S_{\mathrm{R}}, S_{\mathrm{A}}$. Some of the results for normally hyperbolic operators do however extend to the pre-normally hyperbolic case. We have for instance a strict analogue of Lemma 3.3.7.

Lemma 3.3.8. The parametrices $S_{\mathrm{A}}, S_{\mathrm{R}}, S_{\mathrm{F}}$ satisfy

(1) $\operatorname{WF}^{\prime}\left(S_{\mathrm{R}}-S_{\mathrm{A}}\right)=\mathscr{C}$,

(2) $\mathrm{WF}^{\prime}\left(S_{\mathrm{R}}-S_{\mathrm{F}}\right)=\mathscr{C} \cap \mathscr{N}_{-} \times \mathscr{N}_{-}$,

(3) $\mathrm{WF}^{\prime}\left(S_{\mathrm{A}}-S_{\mathrm{F}}\right)=\mathscr{C} \cap \mathscr{N}_{+} \times \mathscr{N}_{+}$.

Proof. (1): The arguments of the proof of (1) of Lemma 3.3.7 apply directly.

(2) and (3): By Lemma 3.3.7, we have the inclusions

$$
\begin{aligned}
\mathrm{WF}^{\prime}\left(S_{\mathrm{R}}-S_{\mathrm{F}}\right) & \subset \mathscr{C} \cap \mathscr{N}_{-} \times \mathscr{N}_{-}, \\
\mathrm{WF}^{\prime}\left(-S_{\mathrm{A}}+S_{\mathrm{F}}\right) & \subset \mathscr{C} \cap \mathscr{N}_{+} \times \mathscr{N}_{+} .
\end{aligned}
$$

In particular, the two sets $\mathrm{WF}^{\prime}\left(S_{\mathrm{R}}-S_{\mathrm{F}}\right)$ and $\mathrm{WF}^{\prime}\left(-S_{\mathrm{A}}+S_{\mathrm{F}}\right)$ are disjoint, hence

$$
\begin{aligned}
\mathrm{WF}^{\prime}\left(S_{\mathrm{R}}-S_{\mathrm{F}}\right) \cup \mathrm{WF}^{\prime}\left(-S_{\mathrm{A}}+S_{\mathrm{F}}\right) & =\mathrm{WF}^{\prime}\left(\left(S_{\mathrm{R}}-S_{\mathrm{F}}\right)+\left(-S_{\mathrm{A}}+S_{\mathrm{F}}\right)\right) \\
& =\mathrm{WF}^{\prime}\left(S_{\mathrm{R}}-S_{\mathrm{A}}\right)=\mathscr{C} .
\end{aligned}
$$

This proves that the inclusions in (3.3.10) are not strict ones.

\subsection{The Hadamard condition}

Duistermaat and Hörmander's original result concerned only parametrices. The key result for applications in Quantum Field Theory is Radzikowski's theorem: it says that in the case of a normally hyperbolic operator $P$ on a globally hyperbolic spacetime $(M, g)$, there is a solution modulo $C^{\infty}$ which is determined uniquely up to smooth functions by its wave front set. The condition on the wave front set is called the microlocal spectrum condition, as it turns out to be a consistent generalization of the properties satisfied by the two-point function of the vacuum state on Minkowski space.

Recall from Section 2.7 that a pair of continuous maps $\Lambda^{(+)}, \Lambda^{(-)}: \mathscr{D}(M, \mathscr{V}) \rightarrow$ 
$\mathscr{D}^{\prime}(M, \mathscr{V})$ are distributional two-point functions of a quasi-free state if

$$
\begin{aligned}
(D) & D \Lambda^{( \pm)}=\Lambda^{( \pm)} D=0 \\
(\text { Pos }) & \beta \Lambda^{( \pm)} \geq 0
\end{aligned}
$$

and

$$
(C A R) \quad \Lambda^{(+)}+\Lambda^{(-)}=\mathrm{i} S, \quad \text { resp. } \quad(C C R) \quad \Lambda^{(+)}-\Lambda^{(-)}=\mathrm{i} S
$$

in the fermionic, respectively bosonic case.

Definition 3.4.1. Let $\Lambda^{(+)}, \Lambda^{(-)}: \mathscr{D}(M, \mathscr{V}) \rightarrow \mathscr{D}^{\prime}(M, \mathscr{V})$ be linear continuous. Then the pair $\Lambda^{(+)}, \Lambda^{(-)}$satisfies the Hadamard condition if

$(\mathrm{Had}) \quad \mathrm{WF}^{\prime}\left(\Lambda^{( \pm)}\right)=\left\{\left(X_{1}, X_{2}\right) \in \mathscr{N}_{ \pm} \times \mathscr{N}_{ \pm}: \quad X_{1} \sim X_{2}\right\}$

Definition 3.4.2. A (neutral or charged, gauge invariant) quasi-free state is a Hadamard state if it has distribitional two-point functions $\Lambda^{( \pm)}$which satisfy the Hadamard condition $(\mathrm{Had})$.

As pointed out in [Hol01], it is in general not true that the condition on $\Lambda^{(+)}$in (Had) follows simply from the condition on $\Lambda^{(-)}$or vice-versa. It is not difficult to see, however, that the two conditions are equivalent if the state is assumed to be invariant under a charge conjugation $\kappa$ which acts on $\mathscr{D}(M, \mathscr{V})$ as an element of $\mathscr{E}(M, \mathscr{L}(\mathscr{V}))$ (cf. Definition 1.5.29). Indeed, in such case the relation between $\Lambda^{(+)}$and $\Lambda^{(-)}$implies that $\operatorname{WF}\left(\Lambda^{(+)}\right)=\operatorname{Exch}\left(\operatorname{WF}\left(\Lambda^{(-)}\right)\right)$. In the sequel, existence of a charge conjugation will not be assumed for the reasons outlined in the introduction to this chapter.

In practice it is often useful to consider the following weaker condition, which turns out to imply $(\mathrm{Had})$ if $\Lambda^{( \pm)}$are distributional two-point functions of a quasi-free state. The definition we use is adapted from [San10], where the charge invariant case is treated.

Definition 3.4.3. We say that $\Lambda^{( \pm)}$satisfy the generalized Hadamard condition if there exists a conic set $\Gamma \subset T^{*}(M \times M) \backslash \mathcal{Z}$ such that $\Gamma \cap-\Gamma=\emptyset$,

$$
\left(X_{1}, X_{2}\right) \in \Gamma \Rightarrow \xi_{1} \in V_{x_{1}+}^{*}
$$

and

$$
\text { (genHad) } \quad \mathrm{WF}^{\prime}\left(\Lambda^{( \pm)}\right) \subset \pm \Gamma .
$$

The next theorem makes the connection between the Hadamard condition and the distinguished parametrices for pre-normally hyperbolic operators. The implication $(1) \Rightarrow(3)$ was initially proved by Radzikowski in the charge-invariant, normally hyperbolic case by Radzikowski in his celebrated paper [Rad96]. The generalization to pre-normally hyperbolic ones (discussed on the example of the Dirac equation) was performed by Hollands in [Hol01], similar results were also obtained in [SV01]. The implementation of ( $\mathrm{genHad}$ ) in the implication $(1) \Rightarrow(3)$ makes uses of the techniques of proof of [Hol01, San10].

Theorem 3.4.4. Let $\Lambda^{(+)}, \Lambda^{(-)}: \mathscr{D}(M, \mathscr{V}) \rightarrow \mathscr{D}^{\prime}(M, \mathscr{V})$ be continuous. The following conditions are equivalent: 
(1) The pair $\Lambda^{(+)}, \Lambda^{(-)}$satisfies $(H a d),(D) \bmod C^{\infty}$ and $(C A R) \bmod C^{\infty}$ (respectively $(C C R) \bmod C^{\infty}$ in the bosonic case);

(2) The pair $\Lambda^{(+)}, \Lambda^{(-)}$satisfies $\left(\right.$genHad) and $(C A R) \bmod C^{\infty}$ (respectively $(C C R)$ $\bmod C^{\infty}$ in the bosonic case);

(3) Define $S^{(+)}:=\mathrm{i}\left(S_{\mathrm{F}}-S_{\mathrm{A}}\right)$ and $S^{(-)}:=\mathrm{i}\left(S_{\mathrm{R}}-S_{\mathrm{F}}\right)$ (respectively $S^{(-)}=\mathrm{i}\left(S_{\mathrm{F}}-\right.$ $\left.S_{\mathrm{R}}\right)$ in the bosonic case). Then

$$
\Lambda^{(+)}=S^{(+)} \bmod C^{\infty}, \quad \Lambda^{(-)}=S^{(-)} \bmod C^{\infty} .
$$

Proof. The implication $(1) \Rightarrow(2)$ is obvious.

$(2) \Rightarrow(3)$ : Recall that by (2), (3) of Lemma 3.3.8, $\mathrm{WF}^{\prime}\left(S^{( \pm)}\right)=\mathscr{C} \cap \mathscr{N}_{ \pm} \times \mathscr{N}_{ \pm}$. Therefore $\Lambda^{( \pm)}-S^{( \pm)}$satisfy (genHad), in particular

$$
\mathrm{WF}^{\prime}\left(\Lambda^{(+)}-S^{(+)}\right) \cap \mathrm{WF}^{\prime}\left(\Lambda^{(-)}-S^{(-)}\right)=\emptyset .
$$

On the other hand, if $(C A R) \bmod C^{\infty}$ holds then by (1) of Lemma 3.3.8

$$
\left(\Lambda^{(+)}-S^{(+)}\right)+\left(\Lambda^{(-)}-S^{(-)}\right)=\mathrm{i}\left(S-\left(S_{\mathrm{A}}-S_{\mathrm{R}}\right)\right)=0 \bmod C^{\infty} .
$$

We conclude that $\Lambda^{(+)}-S^{(+)}$and $\Lambda^{(-)}-S^{(-)}$are smooth. The proof in the bosonic case is analogous.

$(2) \Rightarrow(1): \quad(D) \bmod C^{\infty}$ is obvious, $(H a d)$ follows directly from Lemma 3.3.8, whereas $(C A R) \bmod C^{\infty}\left(\operatorname{resp} .(C C R) \bmod C^{\infty}\right)$ follows from $S=S_{\mathrm{A}}-S_{\mathrm{R}}$.

The next proposition generalizes a well-known result (usually called the local-toglobal theorem) to the case of states which are not necessarily invariant under charge conjugation. The method of proof is similar to the arguments used in [San10].

Proposition 3.4.5. Let $S^{( \pm)}$be defined as in Theorem 3.4.4. Assume $\Lambda^{( \pm)}$satisfy $(P o s) \bmod C^{\infty},(C A R)$ (respectively $\left.(C C R)\right) \bmod C^{\infty}$, and suppose

$$
\mathrm{WF}^{\prime}\left(\Lambda^{(+)}-S^{(+)}\right)=\mathrm{WF}^{\prime}\left(\Lambda^{(-)}-S^{(-)}\right)=\emptyset
$$

above the diagonal $\Delta_{2} \subset M^{\times 2}$. Then $\Lambda^{( \pm)}$satisfy $(H a d)$ and $(D) \bmod C^{\infty}$.

Proof. We can assume with no loss of generality that $\Lambda^{( \pm)}$satisfy (Pos). This means

$$
\left|\left\langle\Lambda^{( \pm)}, f^{\dagger} \otimes g\right\rangle\right|^{2} \leq\left\langle\Lambda^{( \pm)}, f^{\dagger} \otimes f\right\rangle\left\langle\Lambda^{( \pm)}, g^{\dagger} \otimes g\right\rangle, \quad f, g \in \mathscr{D}(M, \mathscr{V}) .
$$

We deduce that

$$
(X, X) \notin \mathrm{WF}^{\prime}\left(\Lambda^{( \pm)}\right) \Rightarrow \begin{aligned}
& \left(X, X_{1}\right) \notin \mathrm{WF}^{\prime}\left(\Lambda^{( \pm)}\right), \quad X_{1} \in T^{*} M \backslash \mathcal{Z}, \\
& \left(X_{2}, X\right) \notin \mathrm{WF}^{\prime}\left(\Lambda^{( \pm)}\right), \quad X_{2} \in T^{*} M \backslash \mathcal{Z} .
\end{aligned}
$$

For definiteness assume $(C A R)$ holds. By Lemma 3.3.8, $\mathrm{WF}^{\prime}\left(S^{( \pm)}\right)=\mathscr{C} \cap \mathscr{N}_{ \pm} \times \mathscr{N}_{ \pm}$, and it follows from (3.4.1) that

$$
(X, X) \in \mathrm{WF}^{\prime}\left(\Lambda^{( \pm)}\right) \Rightarrow(X, X) \in \mathrm{WF}^{\prime}\left(S^{( \pm)}\right) \Rightarrow \xi \in V_{ \pm}^{*}
$$

Therefore using (3.4.2) we see that

$$
\left(X_{1}, X_{2}\right) \in \mathrm{WF}^{\prime}\left(\Lambda^{( \pm)}\right) \Rightarrow \xi_{1} \in V_{x_{1} \pm}^{*}, \xi_{2} \in V_{x_{2} \pm}^{*}
$$

Hence, $\Lambda^{( \pm)}$satisfy (genHad) and the assertion follows by Theorem 3.4.4.

On suitably chosen neighbourhoods of the diagonal $\Delta_{2} \subset M \times M$, it is possible 
to give explicit formulae for the singular terms of $\Lambda^{(+)}$, using a recursive construction traditionally attributed to Hadamard and worked out in detail by subsequent authors (see, e.g., [HM12b] for a recent review). Namely, one considers a set of the form $U \times U$, where $U$ is a causal normal neighbourhood of a given point $x \in M$, i.e., for all $x_{1}, x_{2} \in U$, the exponential map exp : $T_{x} M \rightarrow M$ is required to be a local diffeomorphism at least in a neighbourhood of $J_{-}\left(x_{1}\right) \cap J_{+}\left(x_{2}\right)$. Then, one chooses a global time coordinate function $\tau$ which is increasing towards the future, and on $U \times U$ one considers an ansatz of the form

$$
\begin{aligned}
h_{\epsilon}\left(x_{1}, x_{2}\right)= & \frac{u\left(x_{1}, x_{2}\right)}{4 \pi\left[\sigma\left(x_{1}, x_{2}\right)+\mathrm{i} \epsilon\left(\tau\left(x_{1}\right)-\tau\left(x_{2}\right)\right)+\epsilon^{2}\right]} \\
& +v\left(x_{1}, x_{2}\right) \ln \frac{\sigma\left(x_{1}, x_{2}\right)+\mathrm{i} \epsilon\left(\tau\left(x_{1}\right)-\tau\left(x_{2}\right)\right)+\epsilon^{2}}{\lambda^{2}},
\end{aligned}
$$

where $u$ and $v$ are smooth functions and $\sigma$ stands for the squared geodesic distance, i.e.

$$
\sigma\left(x_{1}, x_{2}\right)= \pm\left(\int_{a}^{b}\left|g_{\mu \nu}(x(\tau)) \frac{d x^{\mu}(\tau)}{d \tau} \frac{d x^{\nu}(\tau)}{d \tau}\right| d \tau\right)^{2}
$$

(here $x(\cdot)$ parametrizes the geodesic from $x_{1}$ to $x_{2}$, in particular $x(a)=x_{1}, x(b)=x_{2}$; the sign depends on whether $x(\cdot)$ is space-like or time-like). The functions $u$ and $v$ are usually written as series involving powers of $\sigma$, and the requirement that $\lim _{\epsilon \backslash 0} h_{\epsilon}$ yields a solution modulo smooth terms gives recursive relations for the coefficients. It was proved that Radzikowski that the distribution $\lim _{\epsilon} \searrow_{0} h_{\epsilon}$, traditionally called the Hadamard parametrix, satisfies indeed the Hadamard condition [Rad96]. Its usefulness, beside the fact that the formulae are very explicit and suitable for practical computations, stems from the fact that it can be expressed in terms of purely geometric quantities ${ }^{2}$.

\subsection{Existence and examples of Hadamard states}

The existence of Hadamard states on a generic globally hyperbolic spacetime was established by Fulling, Narcowich and Wald in [FNW81]. They showed that the problem can always be reduced to spacetimes which possess an ultra-static region, which is sufficiently large to contain a Cauchy surface with its neighbourhood. A Hadamard state can be constructed explicitely on that neighbourhood and then it is propagated to the whole spacetime. One of the features of this method is that one can always obtain pure states as an output. On the other hand, the construction is intrisically non-local and rather non-explicit, which poses problems in practical applications.

An alternative method consists of constructing a sufficiently explicit parametrix for $P$ in terms of pseudo-differential operators, which distinguishes between solutions which propagate with positive and negative frequencies in the wave front set. The first result along these lines is the work of Junker [Jun95], where a construction of Hadamard states for the Klein-Gordon equation is performed for spacetimes with a

${ }^{2}$ This observation can be also made precise in the presence of external potentials, cf. [Zah13]. 
compact Cauchy surface. A more general construction was recently obtained for a class of spacetimes whose metric components are reasonably well-behaved at spatial infinity [GW13]. It is also shown in [GW13] that one can use these results to construct Hadamard states on arbitrary globally hyperbolic spacetimes by a 'cutting and patching' procedure: a partition of unity is employed to reduce the problem to spacetimes with a compact Cauchy surface. This technique is local, one does however not get pure states in general.

Beside the general constructions mentioned above, known examples of Hadamard states include:

(1) passive states for stationary spacetimes (this includes ground- and KMS states) [SV00],

(2) states constructed in [DMP05, Mor08] for a subclass of asymptotically flat vacuum spacetimes at null infinity,

(3) states constructed in [DMP09] for a class of cosmological spacetimes (this includes the Bunch-Davies state on de-Sitter spacetime),

(4) states of low energy for Friedmann-Lemaitre-Robertson-Walker spacetimes [Olb07],

(5) the so-called Unruh state [DMP11],

In the next chapter we will study in more detail stationary states on static spacetimes in the presence of time-independent external potentials. 



\section{CHAPTER 4}

\section{Dirac equation in static external potentials}

In a stationary spacetime, the ground state with respect to the time flow, if exists, provides a distinguished state which generalizes the properties of the Minkowski vacuum. It was proved by Sahlmann and Verch [SV00, SV01] that it satisfies the Hadamard condition (this generalizes earlier results of Fulling, Narcowich and Wald for spin-0 fields on static spacetimes [FNW81]). A different proof was given by Strohmaier, Verch and Wollenberg [SVW02] using the notion of wave front set for distributions with values in a Banach space.

Following [Wro12], we provide a simple proof of this result in the static case, allowing also for time-independent external potentials. The main advantage beside technical simplicity is that the method extends to the case of the Klein-Gordon equation in a strong electric potential, as will be explained in Chapter 5. In this very chapter we focus on the static Dirac equation. We briefly explain how it can be reduced to the form of an evolution equation with self-adjoint generator and we then discuss the construction of stationary Hadamard states.

\subsection{Static spacetimes}

Definition 4.1.1. A spacetime $(M, g)$ is called stationary if there exists a smooth, complete, future-pointing, time-like Killing vector field $\xi$ on $M$. It is called static if in addition $\xi$ can be chosen to be irrotational, i.e., if $M$ can be foliated by hypersurfaces orthogonal to $\xi$.

The definition of a stationary spacetime can be rephrased as follows. First, completeness of $\xi$ means that it induces a flow $\Xi_{t}: \mathbb{R} \times M \rightarrow M$ by

$$
\xi(0, x)=x, \quad d \Xi\left(t, x ; \partial_{t}, 0\right)=\xi(\Xi(t, x)), \quad t \in \mathbb{R} .
$$

Then, the propriety which states that $\xi$ is a Killing vector field is equivalent to invariance of the metric under the flow $\Xi_{t}$, i.e., $\Xi_{t}^{*} g=g$ for all $t \in \mathbb{R}$.

In a static spacetime $(M, g)$, one can choose local coordinates $(t, \mathbf{x})$ such that $\xi=\partial_{t}$ and such that the metric reads

$$
g=-\vartheta(\mathbf{x}) d t^{2}+h_{i j}(\mathbf{x}) d \mathbf{x}^{i} d \mathbf{x}^{i}
$$

where $\vartheta>0$ and $h_{i j}$ are smooth and do not depend on $t$. If in addition $\vartheta$ is bounded from below and from above by positive constants and $(\Sigma, h)$ is a complete Riemannian monifold, then the spacetime $(M, g)$ is globally hyperbolic [Kay78]. 


\subsection{Abstract Dirac equations}

Let us consider a globally hyperbolic static spacetime $M=\mathbb{R} \times \Sigma$ with metric as in (4.1.1) and let $S M$ be a spin structure. We also assume that we are given a potential $A$ and mass term $m$ which do not depend on $t$ and write $A=\left(V, A_{i}\right)$ in the coordinates $\left(t, \mathbf{x}^{i}\right)$. The Dirac operator reads

$$
D=-\gamma^{\mu}\left(\nabla_{\mu}+\mathrm{i} A_{\mu}\right)+m
$$

From Section 2.7 we know that we can multiply from both sides a pre-normally hyperbolic operator $D$ by smooth sections (provided they are invertible and formally self-adjoint) without losing track of the quantities needed for quantization. In particular, this observation allows us to consider instead of $D$ the operator

$$
D^{\prime}:=\mathrm{i} \partial_{t}+H_{(\mathbf{x})},
$$

where $H_{(\mathbf{x})}$ is a differential operator with time-independent coefficients, given by

$$
H_{(\mathbf{x})}=\vartheta^{-1 / 2} \gamma^{0}\left(\mathrm{i} \gamma^{i}\left(\nabla_{i}+\mathrm{i} A_{i}\right)-m\right)+V
$$

It can be checked using Propositions 2.5.4 and 2.7.1 that the Cauchy-surface charge form associated to $D^{\prime}$ concides on test sections with the Hilbert space scalar product in $L^{2}(\Sigma, D M):=L^{2}\left(\Sigma, \mathbb{C}^{2^{[n / 2]}}\right)$, denoted $(\cdot \mid \cdot)$ in what follows. Moreover, $H_{(\mathbf{x})}$ is hermitian on $\mathscr{D}(\Sigma, D M)$, i.e.

$$
\left(u \mid H_{(x)} v\right)=\left(H_{(x)} u \mid v\right), \quad u, v \in \mathscr{D}(\Sigma, D M),
$$

it makes thus sense to ask under what circumstances does it define a self-adjoint operator. As pointed out in [Shi91], essential self-adjointness of $H_{(\mathbf{x})}$ follows directly from the general arguments given in [Che73], which are valid for arbitrary smooth potentials without the need to assume any particular decay of the potentials at infinity.

Proposition 4.2.1. If $A_{i}, V, m$ are smooth and real valued, then the operator $H_{(\mathbf{x})}$ acting on $\mathscr{D}(\Sigma, D M)$ is essentially self-adjoint in the Hilbert space $L^{2}(\Sigma, D M)$.

The closure of $H_{(\mathbf{x})}$, denoted $H$, is traditionally called the Dirac Hamiltonian. Its spectral properties for various classes of potentials are a well studied topic, see e.g. [Tha92], where the flat case is discussed. In particular in the free case, i.e. under the assumptions $(\Sigma, h)=\mathbb{R}^{d}, A \equiv 0$ and $m$ constant, the Dirac Hamiltonian $H$ is self-adjoint with domain $\operatorname{Dom}(H)=H^{1}\left(\mathbb{R}^{d}, \mathbb{C}^{2^{[n / 2]}}\right)$ and its spectrum is the set $\operatorname{sp}(H)=(-\infty,-m] \cup[m, \infty)$.

All summed up together, this allows us to set the static Dirac equation in an abstract framework which is particularly convenient for the quantization. According to our discussion, it is natural to consider pre-normally hyperbolic operators $D$ on static spacetimes which satisfy the following set of assumptions.

Assumption 4.2.2. We assume that

i) $D$ is a differential operator of the form $D=\mathrm{i} \partial_{t}+H_{(\mathbf{x})}$, where $H_{(\mathbf{x})}$ : $\mathscr{D}(\Sigma, \mathscr{V}) \rightarrow \mathscr{D}(\Sigma, \mathscr{V})$ is a differential operator s.t. $\left(H_{(\mathbf{x})}\right)^{2}$ is elliptic. 
Moreover, we assume that there exists a Hilbert space $(\mathcal{H},(\cdot \mid \cdot))$ and a self-adjoint operator $H \in \mathcal{C}(\mathcal{H})$, s.t.

ii) $\mathscr{D}(\Sigma, \mathscr{V})$ is dense in $\mathcal{H}$ and there exists $\beta \in \mathscr{E}\left(M, \mathscr{L}\left(\mathscr{V}, \mathscr{V}^{*}\right)\right)$ s.t.

$$
(u \mid v)=\int_{\Sigma} \beta(u, v) d s, \quad u, v \in \mathscr{D}(\Sigma, \mathscr{V})
$$

iii) $H_{(\mathbf{x})}=H$ on $\mathscr{D}(\Sigma, \mathscr{V})$.

\subsection{Hadamard states}

Suppose that Assumption 4.2.2 is satisfied. Then $\mathrm{i} \partial_{t}+H_{(\mathbf{x})}$ is pre-normally hyperbolic, and the assumption on self-adjointness implies that $D$ is formally self-adjoint w.r.t. $\beta$. We have thus a well defined charge form $Q$ and Cauchy-surface charge form $q$. From the results in Chapter 2 we obtain that:

- The Cauchy-data charge form $q(\cdot, \cdot)$ equals $(\cdot \mid \cdot)$ on $\mathscr{D}(\Sigma, \mathscr{V})$.

- The Cauchy problem

$$
(D) \quad\left\{\begin{array}{l}
D f=0, \quad f \in \mathscr{E}(M, \mathscr{V}) \\
\left.f\right|_{t=0}=\varphi_{0}, \quad \mathscr{D}(\Sigma, \mathscr{V})
\end{array}\right.
$$

is uniquely solved by $f=-\mathrm{i}\left(S \beta^{-1} q\right) \varphi_{0}$.

On the other hand, we certainly know that the same Cauchy problem is uniquely solved by $f=\left(T_{t}\right) \varphi_{0}$, where $T_{t}=\mathrm{e}^{\mathrm{i} t H}$. In order to write the relation between $S$ and $T_{t}$ it is useful to use translation invariance in the time coordinate. Namely, $S \in \mathscr{D}^{\prime}\left(M^{\times 2}, \mathscr{L}(\mathscr{V})\right)$ depends on the time coordinates via the difference $t-t^{\prime}$ only, which means that $S$ is the pullback of a distribution $\tilde{S} \in \mathscr{D}^{\prime}\left(\mathbb{R} \times \Sigma^{\times 2}, \mathscr{L}(\mathscr{V})\right)$ under the map

$$
M \times M \ni\left(t, \mathbf{x}, t^{\prime}, \mathbf{x}^{\prime}\right) \mapsto \tau\left(t, \mathbf{x}, t^{\prime}, \mathbf{x}^{\prime}\right):=\left(t-t^{\prime}, \mathbf{x}, \mathbf{x}^{\prime}\right) \in \mathbb{R} \times \Sigma^{\times 2}
$$

We have then

$$
\begin{aligned}
\langle\tilde{S}, f \otimes \bar{u} \otimes v\rangle & =\mathrm{i} \int_{\mathbb{R}}\left(u \mid T_{t} v\right) f(t) d t \\
& =\mathrm{i}\left(u \mid\left(\mathcal{F}^{-1} f\right)(H) v\right), \quad f \in \mathscr{S}(\mathbb{R}), u, v \in \mathscr{D}(\Sigma, \mathscr{V})
\end{aligned}
$$

where we used a non-unitary convention for the Fourier transform $\mathcal{F}$ and $\left(\mathcal{F}^{-1} f\right)(H)$ is defined by functional calculus.

Since the charge form $q$ concides with the inner product in $\mathcal{H}$ on a dense subspace, the CAR $C^{*}$-algebra of interest is $\operatorname{CAR}(\mathcal{H},(\cdot \mid \cdot))$. For later reference it is convenient to reformulate in the following way the characterization of two-point functions of quasi-free states discussed in Chapter 1.

Proposition 4.3.1. Let $P \in \mathcal{B}(\mathcal{H})$. Then $\lambda:=(\cdot \mid P \cdot)$ is the two-point function of a gauge-invariant quasi-free state on $\operatorname{CAR}(\mathcal{H},(\cdot \mid \cdot))$ iff

$$
P \geq 0, \quad 1-P \geq 0
$$


Moreover, the state is pure iff $P$ is a projection.

If $\mathcal{J} \subset \mathbb{R}$ is a finite union of intervals, we denote $\mathbb{1}_{\mathcal{J}}$ its characteristic function. In the case when $0 \notin \mathrm{sp}(H)$, we have a distinguished projection which satisfies (4.3.3), namely $P=\mathbb{1}_{(0, \infty)}(H)$. It is well known that it corresponds to the unique ground state w.r.t. the time flow and it was proved in [SV00] that it is a Hadamard state.

In order to include the degenerate case $0 \in \operatorname{sp}(H)$ in our discussion, let us consider for a generic interval $\mathcal{J} \subset \mathbb{R}$ the hermitian form

$$
\lambda_{\mathcal{J}}(\cdot, \cdot):=\left(\cdot \mid \mathbb{1}_{\mathcal{J}}(H) \cdot\right) .
$$

Since $1-\mathbb{1}_{\mathcal{J}}=\mathbb{1}_{\mathbb{R} \backslash \mathcal{J}}$ and $H$ is self-adjoint, we immediately obtain by Proposition 4.3.1 that $\lambda_{\mathcal{J}}$ defines a pure quasi-free state iff $\mathcal{J} \cap \operatorname{sp}(H) \subset[0, \infty)$. We will prove in the next section that $\lambda_{\mathcal{J}}$ satisfies the Hadamard condition. As a consequence, any convex combination of two-point functions of the form (4.3.4) is the two-point function of a Hadamard quasi-free state.

4.3.1. Hadamard condition for ground states. As we have seen before, in the static case we can use translation invariance of the causal propagator to write it as $S=\tau^{*} \tilde{S}$, where $\tau$ maps $\left(t, t^{\prime}\right)$ to the time difference $t-t^{\prime}$ and $\tilde{S} \in \mathscr{D}\left(\mathbb{R} \times \Sigma^{\times 2}, \mathscr{V}\right)$. The wave front set of $\tilde{S}$ is determined by the relation

$$
\mathrm{WF}(\tilde{S})=\left\{\left(t, \mathbf{x}, t^{\prime}, \mathbf{x}^{\prime} ; \xi, \mathbf{k},-\xi, \mathbf{k}^{\prime}\right):\left(t-t^{\prime}, \mathbf{x}, \mathbf{x}^{\prime} ; \xi, \mathbf{k}, \mathbf{k}^{\prime}\right) \in \mathrm{WF}(S)\right\} .
$$

Suppose now that we have some distribution $\tilde{\Lambda}^{(+)} \in \mathscr{D}^{\prime}\left(\mathbb{R} \times \Sigma^{\times 2}, \mathscr{V}\right)$ and let us define $\Lambda^{(+)}:=\tau^{*} \tilde{\Lambda}^{(+)}$. Then we have an analogous relation for the wave front sets of $\Lambda^{(+)}$ and $\tilde{\Lambda}^{(+)}$and we can deduce that the pair $\Lambda^{(+)}, \Lambda^{(-)}$satisfies the Hadamard condition $(\mathrm{Had})$ iff

$$
\mathrm{WF}\left(\tilde{\Lambda}^{( \pm)}\right)=\operatorname{WF}(\tilde{S}) \cap \Gamma^{ \pm}
$$

where $\Gamma^{ \pm}:=\left\{\left(\xi, \mathbf{k}, \mathbf{k}^{\prime}\right) \in T^{*}\left(\mathbb{R} \times \Sigma^{\times 2}\right) \backslash \mathcal{Z}: \pm \xi>0\right\}$.

Condition (4.3.5) is implied by a stronger condition on the support of the Fourier transform of $\Lambda^{( \pm)}$, which will turn out to hold in our main case of interest.

First let us recall a basic lemma due to Hörmander.

Lemma 4.3.2 ([Hör83], Lemma 8.1.7). If $u \in \mathscr{S}^{\prime}\left(\mathbb{R}^{p}\right)$, then $\mathrm{WF}(u) \subset \mathbb{R}^{p} \times F$, where

$$
F:=\left\{\lim _{j \rightarrow \infty} \alpha_{j} x_{j}: x_{j} \in \operatorname{supp}(\mathcal{F} u) \subset \mathbb{R}^{p}, \alpha_{j}>0, \lim _{j \rightarrow \infty} \alpha_{j}=0\right\}
$$

and $\mathcal{F}$ denotes the Fourier transform in $\mathbb{R}^{p}$.

We will consider distributions contained in the topological tensor product $\mathscr{S}^{\prime}(\mathbb{R}) \hat{\otimes} \mathscr{D}^{\prime}\left(\Sigma^{\times 2}, \mathscr{L}(\mathscr{V})\right) \subset \mathscr{D}^{\prime}\left(\mathbb{R} \times \Sigma^{\times 2}, \mathscr{L}(\mathscr{V})\right)$, so that it makes sense to apply a Fourier transform in the first argument (thanks to continuity of the Fourier transform in $\left.\mathscr{S}^{\prime}(\mathbb{R})\right)$.

TheOREM 4.3.3. Let $D$ be a differential operator which satisfies i) of Assumption 4.2.2. Suppose that $\Lambda^{( \pm)}$satisfy $(C A R)($ or $(C C R))$ and $(D) \bmod C^{\infty}, \Lambda^{( \pm)}=\tau^{*} \tilde{\Lambda}^{( \pm)}$ 
for some $\tilde{\Lambda}^{( \pm)} \in \mathscr{S}^{\prime}(\mathbb{R}) \hat{\otimes} \mathscr{D}^{\prime}\left(\Sigma^{\times 2}, \mathscr{L}(\mathscr{V})\right)$ and

$$
\begin{aligned}
& \operatorname{supp}\left(\mathcal{F}_{0} \tilde{\Lambda}^{(+)}\right) \subset\left[\alpha_{+}, \infty\right) \times \Sigma^{\times 2} \text { for some } \alpha_{+} \in \mathbb{R}, \\
& \operatorname{supp}\left(\mathcal{F}_{0} \tilde{\Lambda}^{(-)}\right) \subset\left(-\infty, \alpha_{-}\right] \times \Sigma^{\times 2} \quad \text { for some } \alpha_{-} \in \mathbb{R}
\end{aligned}
$$

where $\mathcal{F}_{0}$ is the Fourier transform in the first variable. Then $\Lambda^{( \pm)}$satisfy $(\mathrm{Had})$.

Proof. Assume for the moment that $\Sigma=\mathbb{R}^{d}, \mathscr{V}$ is trivial and $u$ is tempered. Then $\operatorname{supp}\left(\mathcal{F}_{0} \tilde{\Lambda}^{( \pm)}\right) \subset\left[\alpha_{ \pm}, \pm \infty\right) \times \mathbb{R}^{2 d}$ implies $\operatorname{supp}\left(\mathcal{F} \tilde{\Lambda}^{( \pm)}\right) \subset\left[\alpha_{ \pm}, \pm \infty\right) \times \mathbb{R}^{2 d}$. By Lemma 4.3.2 this yields

$$
\operatorname{WF}\left(\tilde{\Lambda}^{( \pm)}\right) \subset \Gamma^{ \pm} \cup \Gamma^{0}
$$

where $\Gamma^{ \pm}=\left(\mathbb{R} \times \Sigma^{\times 2}\right) \times\left((0, \pm \infty) \times \mathbb{R}^{2 d}\right)$ and $\Gamma^{0}=\left(\mathbb{R} \times \Sigma^{\times 2}\right) \times\left(\{0\} \times \mathbb{R}^{2 d}\right)$.

Let us now consider the general case $\tilde{\Lambda}^{( \pm)} \in \mathscr{S}^{\prime}(\mathbb{R}) \hat{\otimes} \mathscr{D}^{\prime}\left(\Sigma^{\times 2}, \mathscr{V}\right)$. Let us fix $s \in$ $\Sigma^{\times 2}$, a (trivializing) coordinate neighbourhood $U \subset \Sigma^{\times 2}$ of $s$, and a test function $\chi \in \mathscr{D}\left(\Sigma^{\times 2}\right)$ s.t. supp $\chi \subset U$ and $\chi \equiv 1$ in a neighbourhood of $s$. By replacing $\tilde{\Lambda}^{( \pm)}$ with the pullback of $(\mathbb{1} \otimes \chi) \tilde{\Lambda}^{( \pm)}$along coordinate maps of $\Sigma$ we are reduced to the previous case and obtain that (4.3.7) holds above $\mathbb{R} \times\{y\}$ for all $y \in \Sigma^{\times 2}$.

Observe that $\tilde{\Lambda}^{( \pm)}$is a parametrix for $D$. Therefore, it is also a parametrix for the elliptic operator $\left(-\mathrm{i} \partial_{t}+H_{(x)}\right) D=\partial_{t}^{2}+\left(H_{(x)}\right)^{2}$, hence $\operatorname{WF}\left(\tilde{\Lambda}^{( \pm)}\right) \cap \Gamma^{0}=\emptyset$. Consequently, (4.3.7) is equivalent to $\operatorname{WF}\left(\tilde{\Lambda}^{( \pm)}\right) \subset \Gamma^{ \pm}$. In particular, $\operatorname{WF}\left(\tilde{\Lambda}^{(+)}\right)$ and $\operatorname{WF}\left(\tilde{\Lambda}^{(-)}\right)$are disjoint. Together with i $\tilde{S}=\tilde{\Lambda}^{(+)}+\tilde{\Lambda}^{(-)} \bmod C^{\infty}$ (or i $\tilde{S}=$ $\left.\tilde{\Lambda}^{(+)}-\tilde{\Lambda}^{(-)} \bmod C^{\infty}\right)$ this entails precisely

$$
\operatorname{WF}\left(\tilde{\Lambda}^{( \pm)}\right)=\operatorname{WF}(\tilde{S}) \cap \Gamma^{ \pm} .
$$

Note that we did not have to assume $\alpha_{+} \geq \alpha_{-}$in Theorem 4.3.3. In fact, the Hadamard condition can be interpreted as a splitting of $S$ into a positive and negative frequency part in an asymptotic sense, i.e. accurate for sufficiently high frequencies.

Proposition 4.3.4. Let $\mathcal{J}$ be an unbounded finite union of intervals s.t. $\mathcal{J} \subset[\alpha, \infty)$ and let $\lambda_{\mathcal{J}}$ be defined as in (4.3.4). Then the associated distributional two-point functions $\Lambda_{\mathcal{J}}^{( \pm)}$satisfy $(\mathrm{Had})$.

Proof. We have $\Lambda_{\mathcal{J}}^{( \pm)}=\tau^{*} \tilde{\Lambda}_{\mathcal{J}}^{( \pm)}$, where

$$
\begin{aligned}
\left\langle\tilde{\Lambda}_{\mathcal{J}}^{(+)}, f \otimes \bar{u} \otimes v\right\rangle & =-\left(u \mid\left(\mathcal{F}^{-1} f\right)(H) \mathbb{1}_{\mathcal{J}}(H) v\right), \\
\left\langle\tilde{\Lambda}_{\mathcal{J}}^{(-)}, f \otimes \bar{u} \otimes v\right\rangle & =-\left(u \mid\left(\mathcal{F}^{-1} f\right)(H)\left(\mathbf{1}-\mathbb{1}_{\mathcal{J}}\right)(H) v\right),
\end{aligned}
$$

for $f \in \mathscr{S}(\mathbb{R}), u, v \in \mathscr{D}(\Sigma, \mathscr{V})$.

We will show that the assumptions of Theorem 4.3.3 are satisfied. By (4.3.2) we have $\mathrm{i} S=\Lambda_{\mathcal{J}}^{(+)}+\Lambda_{\mathcal{J}}^{(-)}$and by construction, $\Lambda_{\mathcal{J}}^{( \pm)}$are bi-solutions. To prove that $\tilde{\Lambda}_{\mathcal{J}}^{( \pm)}$is tempered in the time direction we have to show $\tilde{\Lambda}_{\mathcal{J}}^{( \pm)}: \mathscr{S}(\mathbb{R}) \otimes \mathscr{D}(\Sigma, \mathscr{V}) \otimes \mathscr{D}(\Sigma, \mathscr{V}) \rightarrow \mathbb{C}$ is continuous. By Schwarz inequality and Borel functional calculus for self-adjoint operators, we have

$$
\begin{array}{r}
\left|\left\langle\tilde{\Lambda}_{\mathcal{J}}^{(+)}, f \otimes \bar{u} \otimes v\right\rangle\right|=\left|\left(u \mid\left(\mathcal{F}^{-1} f\right) \mathbb{1}_{\mathcal{J}}(H) v\right)\right| \leq\|u\|\left\|\left(\mathcal{F}^{-1} f\right)(H) \mathbb{1}_{\mathcal{J}}(H)\right\|\|v\| \\
\leq\|u\|\left\|\left(\mathcal{F}^{-1} f\right) \mathbb{1}_{\mathcal{J}}\right\|_{\infty}\|v\| \leq\|u\|\left\|\mathcal{F}^{-1} f\right\|_{\infty}\|v\| .
\end{array}
$$


Convergence of $f$ to 0 in $\mathscr{S}(\mathbb{R})$ implies $\mathcal{F}^{-1} f \rightarrow 0$ in $\mathscr{S}(\mathbb{R})$ and consequently $\left\|\mathcal{F}^{-1} f\right\|_{\infty} \rightarrow 0$. Furthermore, convergence of $u$ (resp. $v$ ) to 0 in $\mathscr{D}(\Sigma, \mathscr{V})$ implies $\|u\| \rightarrow 0$ (resp. $\|v\| \rightarrow 0$ ), hence $\tilde{\Lambda}_{\mathcal{J}}^{(+)}$is continuous. The reasoning for $\tilde{\Lambda}_{\mathcal{J}}^{(-)}$is analogous.

Finally, to see that $\mathcal{F}_{0} \tilde{\Lambda}_{\mathcal{J}}^{( \pm)}$have the required support properties, observe that for any $\varphi \in \mathscr{D}(\mathbb{R})$ with $\operatorname{supp} \varphi \cap \mathcal{J}=\emptyset$, we have

$$
\left\langle\mathcal{F}_{0} \tilde{\Lambda}_{\mathcal{J}}^{(+)}, \varphi \otimes \bar{u} \otimes v\right\rangle=-2 \pi\left(u \mid \varphi(H) \mathbb{1}_{\mathcal{J}}(H) v\right)=-2 \pi\left(u \mid\left(\varphi \cdot \mathbb{1}_{\mathcal{J}}\right)(H) v\right)=0,
$$

and the analogous property holds for $\tilde{\Lambda}_{\mathcal{J}}^{(-)}($with $\mathbb{R} \backslash \mathcal{J}$ instead of $\mathcal{J}$ ). 


\section{CHAPTER 5}

\section{Klein-Gordon equation in static external potentials}

The similarities between the two-component form of the Klein-Gordon equation and the Dirac equation, outlined in Chapter 2, suggest the possibility to treat spin-0 fields in the static case much analogously to the spin- $\frac{1}{2}$ case considered in Chapter 4 . There are however two problems:

- The charge form $q$ associated to the Klein-Gordon equation is not positive and thus, contrary to the Dirac case, it cannot be used to define a Hilbert space.

- If the electric potential $V$ is too strong, there exists no ground state.

The first difficulty can be overcome by considering instead a Krein space, i.e. a Hilbertizable space equipped with a non-necessarily positive hermitian form. Under reasonable assumptions on the potentials it is then possible to express the two-component Klein-Gordon equation as an evolution equation whose generator $B$ is an unbounded operator, formally self-adjoint w.r.t. the hermitian form $q$.

The second problem stems from the fact that for sufficiently strong electric potentials (referred to as overcritical ones) it is impossible to transform the dynamics into an evolution equation whose generator is a self-adjoint operator in the usual Hilbert space sense [Bro83]. This phenomenon, usually called the Klein paradox, is connected to the appearence of non-real eigenvalues in the spectrum of $B$. Although the general argument of Fulling, Narcowich and Wald implies that Hadamard states still exist in that case, it is not clear what property could be used to select a preferred one.

Following [Wro12], we investigate both the subcritical and overcritical case. In analogy to the results of Chapter 4 we show that ground states for the Klein-Gordon equation with subcritical, static, smooth potentials satisfy the Hadamard condition (providing thus an alternative proof for the result of [FNW81]). For a class of overcritical smooth potentials, we still find families of Hadamard states which are ground states after restricting the two-point function to an infinite dimensional subspace. This improves on the result in [Wro12], as the states we construct are gauge-invariant.

In our implementation of Krein space methods, we use extensively results due to Langer, Najman and Tretter [LNT08] and Gérard [Gér12]. One of the crucial assumptions we make is that $B$ belongs to the class of definitizable operators, which admit a functional calculus with particularly good properties.

\subsection{Abstract Klein-Gordon equation}

In analogy to the Dirac equation, we want to consider a more abstract version of the Klein-Gordon equation which emphasizes its spectral properties. 
To this end, let $\mathfrak{h}$ be a Hilbert space and suppose that the Klein-Gordon equation can be written as

$$
\left[\left(\partial_{t}-\mathrm{i} V\right)^{2}+\epsilon^{2}\right] f(t)=0
$$

where $V \in \mathcal{C}(\mathfrak{h})$ is a self-adjoint operator and $\epsilon \in \mathcal{C}(\mathfrak{h})$ is strictly positive. For instance, the Klein-Gordon equation with vanishing potentials on Minkowski space can be brought to this form by setting $\mathfrak{h}=L^{2}\left(\mathbb{R}^{d}\right)$ and $\epsilon=\left(-\Delta+m^{2}\right)^{1 / 2}$ if $m^{2}>0$.

As already anticipated in Chapter 2, we can formally reexpress (5.1.1) as an evolution equation which involves only time derivatives of order one.

$$
\left(\mathrm{i} \partial_{t}+B\right)\left(\begin{array}{l}
f_{0}(t) \\
f_{1}(t)
\end{array}\right)=0, \quad B:=\left(\begin{array}{cc}
V & \mathbf{1} \\
\epsilon^{2} & V
\end{array}\right) .
$$

The one-component and two-component Klein-Gordon equations are then related as follows:

$$
\left(\mathrm{i} \partial_{t}+B\right)\left(\begin{array}{l}
f_{0}(t) \\
f_{1}(t)
\end{array}\right)=0 \Longleftrightarrow\left\{\begin{array}{l}
\left.\left[\left(\partial_{t}-\mathrm{i} V\right)\right)^{2}+\epsilon^{2}\right] f(t)=0 \\
f_{0}(t)=f(t), \quad f_{1}(t)=-\left(\mathrm{i} \partial_{t}+V\right) f(t) .
\end{array}\right.
$$

We have seen in Chapter 2 that the differential expression $D$ which corresponds to $\mathrm{i} \partial_{t}+B$ is pre-normally hyperbolic. Moreover, $D$ is formally self-adjoint w.r.t. the hermitian form

$$
\beta(v, w)=\bar{v}_{0} w_{1}+\bar{v}_{1} w_{0} \quad v, w \in \mathbb{C}^{2} .
$$

and the corresponding charge form equals $q(\cdot, \cdot)=\int_{\Sigma} \beta(\cdot, \cdot) d s$. The situation is thus very similar to the abstract Dirac equation discussed in Section 4.2 , except that the hermitian form $q$ is not positive definite and it cannot be used to define a Hilbert space. The appropriate replacement are Krein spaces. We will see in 5.1.2, how one can build a suitable Krein space equipped with a non-positive hermitian form $[\cdot \mid \cdot]$ which concides with $q(\cdot, \cdot)$ on test functions, and how assign to the formal expression $B$ an operator which is Krein self-adjoint in the appropriate sense

Even though $q(\cdot, \cdot)$ is not positive definite, it is still possible to work in a Hilbert space setting if the potential $V$ is not 'too large'. This can be understood in the following way. Let us introduce the operator

$$
A:=\left(\begin{array}{cc}
0 & \mathbf{1} \\
\epsilon^{2}-V^{2} & 2 V
\end{array}\right)=\left(\begin{array}{cc}
\mathbf{1} & 0 \\
V & \mathbf{1}
\end{array}\right)\left(\begin{array}{cc}
V & \mathbf{1} \\
\epsilon^{2} & V
\end{array}\right)\left(\begin{array}{cc}
\mathbf{1} & 0 \\
-V & \mathbf{1}
\end{array}\right)=\left(\begin{array}{cc}
\mathbf{1} & 0 \\
V & \mathbf{1}
\end{array}\right) B\left(\begin{array}{cc}
\mathbf{1} & 0 \\
-V & \mathbf{1}
\end{array}\right) .
$$

In a formal sense, $A$ is hermitian with respect to the sesquilinear form $[\cdot \mid \cdot]_{\mathrm{en}}$ (the so-called energy inner product), defined as

$$
[u \mid v]_{\mathrm{en}}:=\left(u_{0} \mid\left(\epsilon^{2}-V^{2}\right) v_{0}\right)+\left(u_{1} \mid v_{1}\right)
$$

on suitable elements $u=\left(u_{0}, u_{1}\right), v=\left(v_{0}, v_{1}\right) \in \mathfrak{h} \oplus \mathfrak{h}$. This sesquilinear form is positive if $\epsilon^{2}-V^{2}$ is a well-defined positive operator. In such a case, one uses $[\cdot \mid \cdot]_{\text {en }}$ to define a Hilbert space and it is possible to assign to $A$ a self-adjoint operator. Under a reasonable set of assumptions, the operators $A$ and $B$ have the same spectral theory, therefore one often prefers to work with $A$ instead of $B$.

The quantity $[u \mid u]_{\mathrm{en}}$ is interpreted as energy conserved by the evolution $t \mapsto \mathrm{e}^{\mathrm{i} t A}$ 
and the violation of positivity, occurring when $\epsilon^{2}-V^{2}$ is not positive, is usually called the Klein paradox (see [GMR85, Man88, Ful89] for disambiguation, historical remarks and detailed discussion on the physics of the Klein paradox). In such case it is necessary to work in a Krein space formalism. The properties of the operator $A$, defined on a suitable Krein space, have been investigated by several authors (there is a particularly vast literature on the positive definite case), see [LNT06] and references therein.

We choose to work with the operator $B$ and the inner product $[\cdot \mid \cdot]$ only, motivated by the fact that it is obtained directly from the charge form $q$ and that it allows for a closer analogy with the Dirac case.

The idea of using the operator $B$ for quantization in external potentials dates back to the 1950's [FV58]. Most of the material of this chapter comes from [Wro12]. Many enlightning remarks on quantization in Krein spaces are contained in [SS70].

5.1.1. Operators in Krein spaces. Let us briefly introduce the basic notions from Krein space theory. The standard references are [Bog74, Lan82]. We follow closely the exposition of this subject contained in [Gér12] and focus on the class of so called definitizable Krein self-adjoint operators, which admits a 'smooth functional calculus' and 'Borel functional calculus' with particularly nice properties.

Definition 5.1.1. A Krein space $(\mathcal{K},[\cdot \cdot \cdot])$ consists of a Hilbert space $\mathcal{K}$ with its scalar product $(\cdot \mid \cdot)_{\mathcal{K}}$ and an inner product $[\cdot \mid \cdot]$ on $\mathcal{K}$ (that is a hermitian sesquilinear form), such that $[\cdot \mid \cdot]=(\cdot \mid J \cdot)_{\mathcal{K}}$ for some invertible, self-adjoint $J \in \mathcal{B}(\mathcal{K})$.

Unless stated otherwise, any topological statements refer to the Hilbert space topology of $\mathcal{K}$. In the literature, a more general definition of Krein spaces is often used, which requires $\mathcal{K}$ to be merely a Hilbertizable topological vector space, but this lies away from our case of interest.

Let $A: \operatorname{Dom}(A) \rightarrow \mathcal{K}$ be a densely defined operator. The Krein adjoint $A^{\dagger}$ of $A$ in $(\mathcal{K},[\cdot \cdot \cdot])$ is defined by

$$
\begin{array}{r}
\operatorname{Dom}\left(A^{\dagger}\right):=\{u \in \mathcal{K}:[u \mid A \cdot] \text { is continuous on } \operatorname{Dom}(A)\}, \\
{[u \mid A v]=\left[A^{\dagger} u \mid v\right] \quad \forall u \in \operatorname{Dom}\left(A^{\dagger}\right), v \in \operatorname{Dom}(A) .}
\end{array}
$$

A densely defined operator $A$ is called Krein self-adjoint, respectively Krein unitary if $A^{\dagger}=A$, resp. $A^{\dagger} A=A A^{\dagger}=\mathbf{1}$. It is called Krein positive if

$$
[u \mid A u] \geq 0, \quad u \in \mathcal{K} .
$$

In such case we will write $A \geq 0$ (this is not to be confused with positivity w.r.t. the Hilbert space scalar product, note also that it is not true that $\mathbf{1} \geq 0$ unless the Krein space product [.|.] is positive). We say that $A$ is Krein negative if $-A \geq 0$. Later on we will use the fact that if $B$ is bounded and $A \geq 0$, then also $B^{\dagger} A B \geq 0$.

Proposition 5.1.2. If $P \in \mathcal{B}(\mathcal{K})$ is a Krein self-adjoint and Krein positive projection, then $[u \mid P u]>0$ for all nonzero $u \in \mathcal{K}$. Furthermore, $\operatorname{Ran} P$ with scalar product inherited from $\mathcal{K}$ is a Hilbert space, and its topology coincides with the topology 
induced by $[u \mid u]^{1 / 2}$.

Definition 5.1.3. A Krein self-adjoint operator $A$ is called definitizable if it has non-empty resolvent set and there exists a real polynomial $p(\lambda)$ s.t. $p(A)$ is Krein positive. Such a polynomial is called definitizing for $A$.

It can be shown that a definitizable operator $A$ can be decomposed as $A=A_{1} \oplus A_{2}$, where $\operatorname{sp}\left(A_{1}\right) \subset \mathbb{R}$ and $A_{2}$ is finite-dimensional and Krein self-adjoint. Making use of the fact that the definitizing polynomial $p(\lambda)$ is real, one can characterize $\operatorname{sp}(A)$ as follows.

Proposition 5.1.4. Let $A$ be a definitizable operator. Then $\operatorname{sp}(A) \backslash \mathbb{R}$ consists of finitely many pairs of isolated eigenvalues $\left\{\lambda_{i}, \bar{\lambda}_{i}\right\}$.

5.1.2. Abstract two-component Klein-Gordon equation. Motivated by analogies with the Dirac case, we will consider the following set of assumptions.

Assumption 5.1.5. We assume that:

i) $D$ is a differential operator of the form $D=\mathrm{i} \partial_{t}+B_{(\mathbf{x})}$, where $B_{(\mathbf{x})}$ : $\mathscr{D}(\Sigma, \mathscr{V}) \rightarrow \mathscr{D}(\Sigma, \mathscr{V})$ is a differential operator s.t. $\left(B_{(\mathbf{x})}\right)^{2}$ is elliptic.

Moreover, we assume there exists a Krein space $(\mathcal{K},[\cdot \cdot \cdot])$ and a Krein self-adjoint operator $B \in \mathcal{C}(\mathcal{K})$, s.t.

ii) $\mathscr{D}(\Sigma, \mathscr{V})$ is dense in $\mathcal{K}$ and there exists $\beta \in \mathscr{E}\left(\Sigma, \mathscr{L}\left(\mathscr{V}, \mathscr{V}^{*}\right)\right)$ s.t.

$$
[u \mid v]=\int_{\Sigma} \beta(u, v) d s, \quad u, v \in \mathscr{D}(\Sigma, \mathscr{V})
$$

iii) $B_{(\mathbf{x})}=B$ on $\mathscr{D}(\Sigma, \mathscr{V})$.

iv) $B$ is definitizable and regular at infinity.

In what follows, we give sufficient conditions for Assumption 5.1.5 to hold, making use of results obtained by Langer, Najman and Tretter [LNT08], basing on earlier works (among others) by Veselić [Ves70] and Jonas [Jon88].

As previously, $\mathfrak{h}$ is a Hilbert space with scalar product denoted $(\cdot \mid \cdot), V \in \mathcal{C}(\mathfrak{h})$ is self-adjoint and $\epsilon \in \mathcal{C}(\mathfrak{h})$ is a strictly positive operator. Let us introduce the Hilbert space

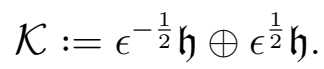

More explicitly, $\epsilon^{-1 / 2} \mathfrak{h}$ is by definition the space $\operatorname{Dom}\left(\epsilon^{\frac{1}{2}}\right)$ with scalar product $\left(\epsilon^{1 / 2}\right.$. $\mid \epsilon^{1 / 2}$. ) and $\epsilon^{1 / 2} \mathfrak{h}$ is the completion of $\mathfrak{h}$ with respect to the norm induced by the scalar product $\left(\epsilon^{-1 / 2} \cdot \mid \epsilon^{-1 / 2}\right.$. $)$. The indefinite inner product $[\cdot \mid \cdot]$ on $\mathcal{K}$ is rigorously defined by

$$
[u \mid v]:=\left(\epsilon^{1 / 2} u_{0} \mid \epsilon^{-1 / 2} v_{1}\right)+\left(\epsilon^{-1 / 2} u_{1} \mid \epsilon^{1 / 2} v_{0}\right)=(u \mid J v)_{\mathcal{K}}
$$

for $u=\left(u_{0}, u_{1}\right), v=\left(v_{0}, v_{1}\right) \in \mathcal{K}$, where $J=\left(\begin{array}{cc}0 & \epsilon^{-1} \\ \epsilon & 0\end{array}\right)$. By invertibility of $J$ It follows that $(\mathcal{K},[\cdot \mid \cdot])$ is a Krein space. Let us define a pair of projections by

$$
\Pi_{ \pm}=\frac{1}{2}(\mathbf{1} \pm J) \in \mathcal{B}(\mathcal{K}) .
$$


Then

$$
\Pi_{ \pm}^{\dagger}=\Pi_{ \pm}, \quad \Pi_{+}+\Pi_{-}=1, \quad \Pi_{\mp} \Pi_{ \pm}=0
$$

and $\pm \Pi_{ \pm} \geq 0$, therefore $\mathcal{K}$ decomposes as a direct sum of two spaces, Ran $\Pi_{+}$and Ran $\Pi_{-}$, which are orthogonal w.r.t. [.|.] and are Hilbert spaces with scalar product respectively $[\cdot \cdot \cdot]$ and $-[\cdot \cdot \cdot]$.

The authors of [LNT08] consider (not necessarily smooth) potentials $V$ satisfying the following assumptions:

Assumption 5.1.6. $V$ and $\epsilon$ are such that

(i) $\operatorname{Dom}(\epsilon) \subset \operatorname{Dom}(V)$,

(ii) $c=V \epsilon^{-1}$ can be decomposed as $c=c_{0}+c_{1}$ with $\left\|c_{0}\right\|<1$ and $c_{1}$ compact,

(iii) $1 \notin \operatorname{sp}_{\mathrm{p}}\left(c^{*} c\right)$.

Under the above assumptions, the operator $B$ in the Hilbert space $\mathcal{K}$ is rigourously defined by

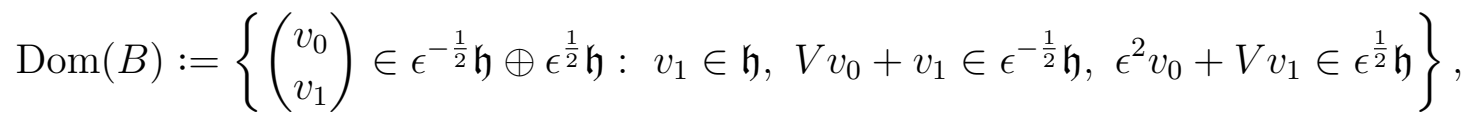

$$
B\left(\begin{array}{c}
v_{0} \\
v_{1}
\end{array}\right):=\left(\begin{array}{c}
V v_{0}+v_{1} \\
\epsilon^{2} v_{0}+V v_{1}
\end{array}\right) .
$$

An important role in the spectral analysis of $B$ is played by the operator $\epsilon^{2}-V^{2}$ defined by

$$
\epsilon^{2}-V^{2}:=\epsilon\left(\mathbf{1}-c^{*} c\right) \epsilon, \quad \operatorname{Dom}\left(\epsilon^{2}-V^{2}\right):=\left\{w \in \epsilon^{-1} \mathfrak{h}: \quad\left(\mathbf{1}-c^{*} c\right) w \in \epsilon^{-1} \mathfrak{h}\right\} .
$$

Let us note that part (iii) of Assumption 5.1.6 is equivalent to $0 \notin \operatorname{sp}_{\mathrm{p}}(B)$. This simplifies much the discussion presented later on, but is not an essential assumption, see [GGH12] for new developments in that direction. The following theorem summarizes the spectral properties of the operator $B$.

TheOrem 5.1.7 ([LNT08]). Suppose that Assumption 5.1.6 is satisfied for $c=c_{0}+c_{1}$ with $\left\|c_{0}\right\|<1$ and $c_{1}$ compact, and let $B$ be the operator defined by 5.1.4. Then:

(1) The operator $B$ is definitizable in the Krein space $(\mathcal{K},[\cdot \cdot \cdot])$ and is regular at $\infty$. Consequently, $B$ is the generator of a strongly continuous group of Krein unitaries $\left\{T_{t}\right\}_{t \in \mathbb{R}}$.

(2) The essential spectrum $\operatorname{sp}_{\text {ess }}(B)$ is real and $\operatorname{sp}_{\text {ess }}(B) \cap(-\alpha, \alpha)=\emptyset$, where $\alpha:=\left(1-\left\|c_{0}\right\|\right) \mu$.

(3) Assume $\mathcal{J} \subset[0, \infty)$ (resp. $\mathcal{J} \subset(-\infty, 0])$ is admissible for $B$. Then, $\mathbb{1}_{\mathcal{J}}(B)$ is Krein positive (resp. Krein negative) iff $\overline{\mathcal{J}} \cap \operatorname{sp}_{\text {cr }}(B)=\emptyset$.

(4) If $c_{1}=0$, then $B$ has no complex eigenvalues.

(5) If $\epsilon^{2}-V^{2}$ is strictly positive, then $\operatorname{sp}(B) \subset \mathbb{R}$ and $\operatorname{sp}_{\text {cr }}(B)=\emptyset$.

As a special case, if $\Sigma=\mathbb{R}^{d}, m>0$ and $A_{i}(\mathbf{x}) \equiv 0$ for $i=1, \ldots, d$, the operator $\epsilon$ equals $\left(-\Delta+m^{2}\right)^{1 / 2}$ with domain $W_{2}^{1}\left(\mathbb{R}^{d}\right)$. Then, one can give explicit examples of classes of potentials $V$ satisfying the assumptions of Theorem 5.1.7. 
Proposition 5.1 .8 ([LNT08]). Let $d \geq 3$. Parts (i)-(ii) of Assumption 5.1.6 are satisfied if $\epsilon=\left(-\Delta+m^{2}\right)^{1 / 2}$ with $m>0$ and $V=V_{0}+V_{1}$, where $V_{1} \in L^{p}\left(\mathbb{R}^{d}\right)$ with $d \leq p<\infty$, and one of the following holds:

(1) $V_{0} \in L^{\infty}\left(\mathbb{R}^{d}\right)$ with $\left\|V_{0}\right\|_{\infty}<m$;

(2) $V_{0}(\mathbf{x})=\gamma /|\mathbf{x}|, \mathbf{x} \in \mathbb{R}^{d} \backslash\{0\}$, with $\gamma \in \mathbb{R}$ s.t. $|\gamma|<(d-2) / 2$.

5.1.3. Quasi-free states. Suppose we have a differential operator $D$ such that Assumption 5.1.5 is satisfied and which has advanced/retarded fundamental solutions. The assumption on self-adjointness implies that $D$ is formally self-adjoint w.r.t. $\beta$. We have thus a well defined charge form $Q$ and Cauchy-surface charge form $q$. From the results in Chapter 2 we obtain that:

- The Cauchy-data charge form $q(\cdot, \cdot)$ equals $[\cdot \mid \cdot]$ on $\mathscr{D}(\Sigma, \mathscr{V})$.

- The Cauchy problem

$$
(D) \quad\left\{\begin{array}{l}
D f=0, \quad f \in \mathscr{E}(M, \mathscr{V}) \\
\left.f\right|_{t=0}=\varphi_{0}, \quad \mathscr{D}(\Sigma, \mathscr{V})
\end{array}\right.
$$

is uniquely solved by $f=-\mathrm{i}\left(S \beta^{-1} q\right) \varphi_{0}$, where $S$ is the causal propagator for $D$.

Since on the other hand, the same Cauchy problem is uniquely solved by $f=\left(T_{t}\right) \varphi_{0}$, we have

$$
\langle\tilde{S}, f \otimes \bar{u} \otimes v\rangle=\mathrm{i} \int_{\mathbb{R}}\left(u \mid T_{t} v\right) f(t) d t \quad f \in \mathscr{S}(\mathbb{R}), u, v \in \mathscr{D}(\Sigma, \mathscr{V}) .
$$

Since the charge form $q$ concides with $[\cdot \mid \cdot]$ on a dense subspace of $\mathcal{K}$, the CCR $C^{*}$-algebra of interest is $\operatorname{CCR}(\mathcal{K},[\cdot \mid \cdot])$. We can reformulate in the following way the characterization of two-point functions of quasi-free states discussed in Chapter 1.

Proposition 5.1.9. Let $P \in \mathcal{B}(\mathcal{K})$. Then $\lambda:=[\cdot \mid P \cdot]$ is the two-point function of a gauge-invariant quasi-free state on $\operatorname{CCR}(\mathcal{K},[\cdot \mid \cdot])$ iff

$$
P \geq 0, \quad \mathbf{1}-P \leq 0
$$

in the sense of Krein space positivity. Moreover, the state is pure iff $P$ is a projection.

If only the first condition $P \geq 0$ is satisfied, then we still obtain a quasi-free state, it will however not be gauge-invariant.

By analogy to the Dirac case, it would be desirable to define a pure quasi-free state by taking $P=\mathbb{1}_{\mathcal{J}}(B)$, where $\mathcal{J}$ is $(0, \infty)$ or $[0, \infty)$ and $\mathbb{1}_{\mathcal{J}}(B)$ should be defined by some generalization of the functional calculus for self-adjoint operators specially adapted to the Krein self-adjoint case. In the next section we will introduce the functional calculus of Langer and Jonas which allows to define spectral projections of a definitizable Krein self-adjoint operator, restricted however to a certain class of intervals which does not always contain the full half-line. 


\subsection{Definitizable operators}

5.2.1. Smooth functional calculus for definitizable operators. We quote first the adaptation of the functional calculus of Davies [Dav95] to definitizable operators in Krein spaces proposed by Gérard [Gér12], omitting the explicit constructions and proofs. This functional calculus is available for classes of smooth functions decreasing fast enough at $\infty$ :

For $\rho \in \mathbb{R}$, denote $S^{\rho}(\mathbb{R})$ the space of functions $f$ such that

$$
\forall_{\alpha \in \mathbb{N}} \exists_{C_{\alpha} \geq 0}:\left|f^{(\alpha)}(\lambda)\right| \leq C_{\alpha}\langle\lambda\rangle^{\rho-\alpha},
$$

equipped with the semi-norms $\|f\|_{m}:=\sup _{\lambda \in \mathbb{R}, \alpha \leq m}\left|\langle\lambda\rangle^{-\rho+\alpha} f^{(\alpha)}(\lambda)\right|$. Here, $f^{(\alpha)}$ denotes the derivative of order $\alpha$ of $f$ and $\langle\lambda\rangle:=\left(1+\lambda^{2}\right)^{1 / 2}$. Note that $\mathscr{S}(\mathbb{R}) \subset S^{\rho}(\mathbb{R})$ for all $\rho \in \mathbb{R}$.

For $f \in S^{\rho}(\mathbb{R})$, define

$$
\tilde{f}(x+\mathrm{i} y):=\left(\sum_{r=0}^{N} f^{(r)}(x) \frac{(\mathrm{i} y)^{r}}{r !}\right) \chi\left(\frac{y}{\delta\langle x\rangle}\right),
$$

where $N$ is some fixed integer, $\delta>0$ and $\chi \in \mathscr{D}(\mathbb{R})$ with $\chi(s) \equiv 1$ for $|s| \leq \frac{1}{2}$ and $\chi(s) \equiv 0$ for $|s| \geq 1$. A function defined this way is called an almost analytic extension of $f$. It satisfies

$$
\left.\tilde{f}\right|_{\mathbb{R}}=f, \quad\left|\frac{\partial \tilde{f}(z)}{\partial \bar{z}}\right| \leq C\langle\operatorname{Re} z\rangle^{\rho-N-1}|\operatorname{Im} z|^{N} .
$$

Proposition 5.2.1 ([Gér12], B.8). Let $A$ be a definitizable operator. Let $\rho<-1$, $f \in S^{\rho}(\mathbb{R})$ and let $\tilde{f}$ be given by (5.2.1). Then for sufficiently high $N$ the integral

$$
f(A):=\frac{1}{2 \pi \mathrm{i}} \int_{\mathbb{C}} \frac{\partial \tilde{f}}{\partial \bar{z}}(A-z)^{-1} d z \wedge d \bar{z} .
$$

is norm convegent in $\mathcal{B}(\mathcal{K})$ and does not depend on the choice of $\chi, \delta, N$. The map $S^{\rho}(\mathbb{R}) \ni f \mapsto f(A) \in \mathcal{B}(\mathcal{K})$ is a homomorphism of algebras and

$$
\begin{gathered}
f(A)^{\dagger}=\bar{f}(A), \\
\|f(A)\| \leq C_{A}\|f\|_{m}, \quad \text { for some } m \in \mathbb{N} .
\end{gathered}
$$

5.2.2. Borel functional calculus. A Borel functional calculus is also available, up to some restrictions on both the operators and the class of functions [Lan82, Gér12]. There, a crucial role is played by the set of critical points $\mathrm{sp}_{\mathrm{cr}}(A)$, defined as follows.

Definition 5.2.2. Let $A$ be a definitizable operator. The set

$$
\mathrm{sp}_{\mathrm{cr}}(A):=\bigcap_{p} p^{-1}(\{0\}) \cap \mathrm{sp}(A) \cap \mathbb{R}
$$

is called the set of critical points of $A$, where the intersection is taken over all definitizing polynomials for $A$. 
It is shown in [Gér12, B.10], that there is an operator-valued measure $\mu$ such that

$$
f(A)=\int_{\mathbb{R}} f(t) d \mu(t)
$$

for each $f \in \mathscr{D}(\mathbb{R})$ with $\operatorname{supp} f \cap \operatorname{sp}_{\text {cr }}(A)=\emptyset$. A construction of such measure $\mu$ is described in [Lan82], we will use this particular choice without giving a more explicit characterization.

Definition 5.2.3. A finite union of intervals $\mathcal{J} \subset \mathbb{R}$ is called admissible for $A$ if its boundary $\partial \mathcal{J}$ contains no critical point of $A$.

Let $\mathcal{J} \subset \mathbb{R}$ be admissible for $A$. We denote by $\mathfrak{B}_{A}(\mathcal{J})$ the ${ }^{*}$-algebra of bounded Borel functions on $\mathcal{J}$ which are locally constant near $\operatorname{sp}_{\text {cr }}(A)$.

Theorem 5.2.4 ([Gér12], B.11). Let $\mathcal{J} \subset \mathbb{R}$ be a bounded admissible finite union of intervals for a definitizable operator $A$ and let $g \in \mathfrak{B}_{A}(\mathcal{J})$. Decompose $g=g_{0}+\sum_{i} g_{i}$, where $g_{0} \in \mathfrak{B}_{A}(\mathcal{J})$ is such that $\operatorname{supp} g_{0} \cap \operatorname{sp}_{\text {cr }}(A)=\emptyset$ and $g_{i} \in \mathscr{D}(\mathbb{R})(i=1, \ldots, N$; $N<\infty)$. Set

$$
g(A):=\sum_{i=1}^{N} g_{i}(A)+\int_{\mathbb{R}} g_{0}(t) d \mu(t),
$$

where $g_{i}(A)$ is defined via smooth functional calculus. Then $g(A)$ is a well-defined operator in $\mathcal{B}(\mathcal{K})$ and the definition does not depend on the decomposition of $g$. The map

$$
\mathfrak{B}_{A}(\mathcal{J}) \ni g \mapsto g(A) \in \mathcal{B}(\mathcal{K})
$$

is a homomorphism of ${ }^{*}$-algebras such that $g(A)^{\dagger}=\bar{g}(A)$.

We use the Borel functional introduced in Theorem 5.2.4 to define spectral projections $\mathbb{1}_{\mathcal{J}}(A)$, where $\mathcal{J}$ is bounded admissible and we recall that $\mathbb{1}_{\mathcal{J}} \in \mathfrak{B}_{A}(\mathcal{J})$ denotes the characteristic function of $\mathcal{J}$. Equivalently, one could use the construction of spectral projections described in [Lan82]. To discuss generalizations for larger classes of intervals, one makes the following definition.

Definition 5.2.5. Let $A$ be a definitizable operator. A point $c \in \operatorname{sp}_{\mathrm{cr}}(A)$ is said to be a regular critical point of $A$ if $\mathbb{1}_{[c-\varepsilon, c+\varepsilon]}(A)$ converges in the strong operator topology as $\varepsilon \searrow 0$. Otherwise, it is said to be a singular critical point. We say that a definitizable operator $A$ is regular at infinity if $\mathbb{1}_{[-L, L]}(A)$ converges in the strong operator topology as $L \rightarrow \infty$.

Let us note that in the literature, a convention where $\infty$ is by definition in $\operatorname{sp}_{\mathrm{cr}}(A)$ is often employed and one speaks of $\infty$ being a 'regular critical point' instead. It is natural to adopt the following notation:

Definition 5.2.6. Let $A$ be a definitizable operator and let $\mathcal{J}$ be a finite sum of bounded intervals such that no singular critical points of $A$ intersects $\partial \mathcal{J}$. We define

$$
\mathbb{1}_{\mathcal{J}}(A):=\mathrm{s}-\lim _{\varepsilon \searrow 0} \mathbb{1}_{\mathcal{J}(\varepsilon)}(A), \quad \mathcal{J}(\varepsilon):=\mathcal{J} \backslash\left(\bigcup_{c \in \mathrm{sp}_{\mathrm{cr}}(A)}[c-\varepsilon, c+\varepsilon]\right)
$$


Definition 5.2.7. Let $A$ be a definitizable operator, regular at infinity, and let $\mathcal{J}$ be a finite sum of intervals such that no singular critical points of $A$ intersects $\partial \mathcal{J}$. If $\mathcal{J}$ is not bounded, we define

$$
\mathbb{1}_{\mathcal{J}}(A):=\mathrm{s}-\lim _{L \rightarrow \infty} \mathbb{1}_{\mathcal{J} \cap[-L, L]}(A) .
$$

Proposition 5.2.8. Let $A$ be a definitizable operator and let $\mathcal{J}, \mathcal{J}^{\prime}$ be finite sums of bounded intervals such that no singular critical points of $A$ intersects $\partial \mathcal{J}$. Let $f \in S^{\rho}(\mathbb{R})$ with $\rho>-1$ and let $f(A)$ be defined by smooth functional calculus. Then:

(1) $\mathbb{1}_{\mathcal{J}}(A)^{\dagger}=\mathbb{1}_{\mathcal{J}}(A)$

(2) $\mathbb{1}_{\mathcal{J}}(A) \mathbb{1}_{\mathcal{J}^{\prime}}(A)=\mathbb{1}_{\mathcal{J} \cap \mathcal{J}^{\prime}}(A)$,

(3) if $\operatorname{supp} f \cap \overline{\mathcal{J}}=\emptyset$ then $f(A) \mathbb{1}_{\mathcal{J}}(A)=0$.

(4) if $\operatorname{supp} f \subset \overline{\mathcal{J}}$ then $f(A) \mathbb{1}_{\mathcal{J}}(A)=f(A)$.

Moreover, if $A$ is regular at infinity, this extends to unbounded $\mathcal{J}$ as well.

Proof. Properties (1)-(2) are direct consequences of Theorem 5.2.4. To prove properties (3)-(4) it suffices to consider $f \in \mathscr{D}(\mathbb{R})\left(\mathscr{D}(\mathbb{R})\right.$ being dense in $\left.S^{\rho}(\mathbb{R})\right)$. For such functions the smooth and Borel functional calculus coincide and using the latter we get

$$
f(A)=\left(f \cdot \mathbb{1}_{\text {supp } f}\right)(A)=f(A) \mathbb{1}_{\text {supp } f}(A)
$$

and one uses property (2) to get (3)-(4). The last assertion follows, as properties (1)-(4) are preserved by the strong operator limit (5.2.7).

5.2.3. One-parameter groups generated by definitizable operators. The following property of definitizable operators which are regular at infinity is essential for our purpose (see [LNT08] for a more complete discussion).

Proposition 5.2.9. Let $A$ be definitizable and regular at infinity. Then, it is the generator of a strongly continuous one-parameter group of Krein unitaries $\left\{T_{t}\right\}_{t \in \mathbb{R}}$, i.e.

$$
A x=\lim _{t \rightarrow 0} \frac{T_{t} x-x}{\mathrm{i} t} \quad \forall x \in \operatorname{Dom}(A) .
$$

Let us now investigate the relation between $T_{t}$ and operators defined by function calculi for $A$. In doing so, one has to take into account that $T_{t}$ contains all the information about eventual complex eigenvalues of $A$, but this not the case for an operator $f(A)$ defined by smooth functional calculus. We illustrate this in Proposition 5.2.10.

First, let us introduce the projection corresponding to the non-real part of the spectrum of $A$. Let $E(\lambda, A)$ denote the Riesz projection relative to an isolated eigenvalue $\lambda \in \operatorname{sp}(A)$. Define

$$
\mathbb{1}^{\mathbb{C} \backslash \mathbb{R}}(A):=\sum_{\lambda \in \operatorname{sp}(A), \operatorname{Im} \lambda>0} E(\lambda, A)+E(\bar{\lambda}, A) .
$$

A standard result from Krein space theory says that $\left[u \mid \mathbb{1}^{\mathbb{C} \backslash \mathbb{R}}(A) u\right]=0$ for all $u \in$ 
$\operatorname{Ran} \mathbb{1}^{\mathbb{C} \backslash \mathbb{R}}(A)$.

Proposition 5.2.10. Let $A$ be definitizable and regular at infinity. Denote $\left\{T_{t}\right\}_{t \in \mathbb{R}}$ the one-parameter group it generates. For any $f \in \mathscr{D}(\mathbb{R})$, one has

$$
\frac{1}{\sqrt{2 \pi}} \int_{\mathbb{R}} d t f(t) T_{t}\left(\mathbf{1}-\mathbb{1}^{\mathbb{C} \backslash \mathbb{R}}(A)\right)=\left(\mathcal{F}^{-1} f\right)(A),
$$

where $\left(\mathcal{F}^{-1} f\right)(A)$ is defined via smooth functional calculus.

Proof. by [ABHN11, Theorem 3.12.2], $T_{t}$ is the inverse Laplace transform of the resolvent of i $A$, i.e.

$$
T_{t}=\mathrm{s}-\lim _{k \rightarrow \infty} \frac{1}{2 \pi \mathrm{i}} \int_{-k}^{k} \mathrm{e}^{(\mu+\mathrm{i} s) t}(A+(\mathrm{i} \mu-s) \mathbf{1})^{-1} d s
$$

for sufficiently large $\mu>0$. by writing the same equality for $T(-t)$ and taking the Krein adjoint, we get also

$$
T_{t}=\mathrm{s}-\lim _{k \rightarrow \infty} \frac{(-1)}{2 \pi \mathrm{i}} \int_{-k}^{k} \mathrm{e}^{(-\mu+\mathrm{i} s) t}(A-(\mathrm{i} \mu-s) \mathbf{1})^{-1} d s .
$$

Using (5.2.11) we get that $\int_{0}^{\infty} d t f(t) T_{t}$ equals

$$
\frac{(-1)}{2 \pi \mathrm{i}} \int_{0}^{\infty} d t \int_{-\infty}^{\infty} d s f(t) \mathrm{e}^{(-\mu+\mathrm{i} s) t}(A-(\mathrm{i} \mu-s) \mathbf{1})^{-1} .
$$

by the Riesz-Dunford calculus,

$$
(A-w \mathbf{1})^{-1}\left(\mathbb{1}^{\mathbb{C} \backslash \mathbb{R}}(A)-\mathbf{1}\right)=\frac{1}{2 \pi \mathrm{i}} \int_{\gamma(w)}(w-z)^{-1}(A-z \mathbf{1})^{-1} d z,
$$

where $\gamma(w):=\gamma_{0}(w) \cup \gamma_{1} \cup \overline{\gamma_{1}}, \gamma_{0}(w)$ is a circle in rs $(A)$ which surrounds $w \in \mathbb{C}$ and $\gamma_{1}$ is a circle in $\operatorname{rs}(A) \cap\{z: \operatorname{Im} z>0\}$ which surrounds $\operatorname{sp}(A) \cap\{z: \operatorname{Im} z>0\}$. Hence

$$
\begin{array}{r}
\left(5.2 .13 \neq \frac{-1}{(2 \pi \mathrm{i})^{2}} \int_{0}^{\infty} d t \int_{-\infty}^{\infty} d s \int_{\gamma(s+\mathrm{i} \mu)} d z f(t) \mathrm{e}^{(-\mu+\mathrm{i} s) t}(s+\mathrm{i} \mu-z)^{-1}(A-z \mathbf{1})^{-1}\right. \\
=\frac{-1}{(2 \pi \mathrm{i})^{2}} \int_{0}^{\infty} d t \int_{-\infty+\mathrm{i} \mu}^{\infty+\mathrm{i} \mu} d w \int_{\gamma(w)} d z f(t) \mathrm{e}^{\mathrm{i} w t}(w-z)^{-1}(A-z \mathbf{1})^{-1} .
\end{array}
$$

We claim that the contour $\gamma(w)$ can be replaced by $\eta(\epsilon):=(\mathbb{R}+\mathrm{i} \epsilon) \cup(\mathbb{R}-\mathrm{i} \epsilon)$ (clockwise), where $\epsilon>0$ is arbitrarily small. To this end we have to prove that the respective integral over two half-circles (in the $z$ variables) with center $\mathrm{i} \epsilon,-\mathrm{i} \epsilon$ and radius $R$ vanishes as $R \rightarrow \infty$. Indeed, we can use that $\left\|(A-z \mathbf{1})^{-1}\right\|$ is $O\left(|\operatorname{Im} z|^{-1}\right)$ for large $|\operatorname{Im} z|$ (as follows from the Hille-Yosida theorem) to show that the integral over $z$ is $O\left(R^{-1} \ln R\right)$. We have

$$
\frac{-1}{(2 \pi \mathrm{i})^{2}} \int_{0}^{\infty} d t \int_{-\infty+\mathrm{i} \mu}^{\infty+\mathrm{i} \mu} d w \int_{\eta(\epsilon)} d z f(t) \mathrm{e}^{\mathrm{i} w t}(w-z)^{-1}(A-z \mathbf{1})^{-1}
$$




$$
=\frac{(-1)}{2 \pi \mathrm{i}} \int_{0}^{\infty} d t \int_{\eta(\epsilon)} d z f(t) \mathrm{e}^{\mathrm{i} z t}(A-z \mathbf{1})^{-1} .
$$

Analogously, using (5.2.10) instead of (5.2.11), one finds

$$
\int_{-\infty}^{0} d t f(t) T_{t}\left(\mathbf{1}-\mathbb{1}^{\mathbb{C} \backslash \mathbb{R}}(A)\right)=\frac{(-1)}{2 \pi \mathrm{i}} \int_{-\infty}^{0} d t \int_{\eta(\epsilon)} d z f(t) \mathrm{e}^{\mathrm{i} z t}(A-z \mathbf{1})^{-1} .
$$

Thus, denoting $g:=\mathcal{F}^{-1} f$,

$$
\frac{1}{\sqrt{2 \pi}} \int_{\mathbb{R}} d t f(t) T_{t}\left(\mathbf{1}-\mathbb{1}^{\mathbb{C} \backslash \mathbb{R}}(A)\right)=\frac{(-1)}{2 \pi \mathrm{i}} \int_{\eta(\epsilon)} g(z)(A-z \mathbf{1})^{-1} .
$$

On the other hand, to evaluate the RHS of (5.2.9), observe that $g$ is an entire function and it consequently admits an almost analytic extension of the form

$$
\tilde{g}(z):=g(z) \chi_{0}(z), \quad \chi_{0}(x+\mathrm{i} y):=\chi(y /(\delta\langle x\rangle)),
$$

where $\chi$ and $\delta$ are as in (5.2.1). Therefore,

$$
\begin{aligned}
(5 . \text { g. (15) ) }) & =\frac{1}{2 \pi \mathrm{i}} \int_{\mathbb{C}} \frac{\partial \tilde{g}}{\partial \bar{z}}(z)(A-z \mathbf{1})^{-1} d z \wedge d \bar{z}=\lim _{\epsilon \searrow 0} \frac{1}{2 \pi \mathrm{i}} \int_{C_{\epsilon}} \frac{\partial \tilde{g}}{\partial \bar{z}}(z)(A-z \mathbf{1})^{-1} d z \wedge d \bar{z} \\
& =\lim _{\epsilon \searrow 0} \frac{1}{2 \pi \mathrm{i}} \int_{\partial C_{\epsilon}} \tilde{g}(z)(A-z \mathbf{1})^{-1} d z=\lim _{\epsilon \searrow 0} \frac{1}{2 \pi \mathrm{i}} \int_{\partial C_{\epsilon}} g(z) \chi_{0}(z)(A-z \mathbf{1})^{-1} d z,
\end{aligned}
$$
hence

where $C_{\epsilon}:=\operatorname{supp} \chi_{0} \cap\{z:|\operatorname{Im} z|>\epsilon\}$. The last integral does not depend on $\epsilon$,

$$
g(A)=\frac{1}{2 \pi \mathrm{i}} \int_{\bar{\eta}(\epsilon)} g(z)(A-z \mathbf{1})^{-1}=\frac{(-1)}{2 \pi \mathrm{i}} \int_{\eta(\epsilon)} g(z)(A-z \mathbf{1})^{-1} .
$$

\subsection{Hadamard states}

Suppose now that Assumption 5.1.5 is satisfied. In particular, we have a definitizable operator $B$ which is regular at $\infty$. Recall also that we have a pair of Krein self-adjoint projections $\Pi_{ \pm}$such that $\pm \Pi_{ \pm} \geq 0$.

Suppose that $\operatorname{sp}_{\mathrm{cr}}(B)$ is contained in the point spectrum of $B$. Then if we set $\mathcal{J}_{ \pm}:=(0, \pm \infty) \backslash \operatorname{sp}_{\mathrm{cr}}(B)$, the projections $\mathbb{1}_{\mathcal{J}_{ \pm}}(B)$ are well defined by Borel functional calculus. Let us define a sesquilinear form $\lambda$ by $\lambda(\cdot, \cdot):=[\cdot \mid P \cdot]$, where

$$
P:=\mathbb{1}_{\mathcal{J}_{+}}(B)+\left(\mathbf{1}-\mathbb{1}_{\mathcal{J}_{-} \cup \mathcal{J}_{+}}(B)\right) \Pi_{+}\left(\mathbf{1}-\mathbb{1}_{\mathcal{J}_{-} \cup \mathcal{J}_{+}}(B)\right) .
$$

Then $P \geq 0$ and we also have

$$
\mathbf{1}-P=\mathbb{1}_{\mathcal{J}_{-}}(B)+\left(\mathbf{1}-\mathbb{1}_{\mathcal{J}_{-} \cup \mathcal{J}_{+}}(B)\right) \Pi_{-}\left(\mathbf{1}-\mathbb{1}_{\mathcal{J}_{-} \cup \mathcal{J}_{+}}(B)\right) \leq 0,
$$

therefore by Proposition 5.1.9, $\lambda$ is the two-point function of a gauge-invariant quasifree state on $\operatorname{CCR}(\mathcal{K},[\cdot \mid \cdot])$. It makes sense to distinguish between the following three cases:

(1) Subcritical case. If $\epsilon^{2}-V^{2} \geq 0$ then $\operatorname{sp}_{\mathrm{cr}}(B)=\emptyset$ and $P=\mathbb{1}_{(0, \infty)}, \mathbf{1}-P=$ $\mathbb{1}_{(-\infty, 0)}$ and one can show that in that case $\lambda$ is the two-point function of the unique ground state. 
(2) Overcritical case, $\pm m \notin \mathrm{sp}_{\mathrm{cr}}(B)$. The condition $\pm m \notin \mathrm{sp}_{\mathrm{cr}}$ implies that there is no critical point embedded in the continuous spectrum of $B$ [LNT08]. Thus, the definition of $\lambda$ stated above makes sense and it will be shown that the corresponding quasi-free state is Hadamard. The role of the term with $\Pi_{+}$in $(5.3 .1)$ is to select the positive frequency part in the finite dimensional space corresponding to complex eigenvalues or critical points. A priori it is possible to do this using a different projection with properties analogous to $\Pi_{+}$, so this part of the definition is to some extent ambiguous.

(3) Overcritical case, $-m$ or $m \in \mathrm{sp}_{\mathrm{cr}}(B)$. In that case $\mathbb{1}_{\mathcal{J}_{ \pm}}(B)$ is ill-defined. Although it is possible to define a state by removing from the intervals $\mathcal{J}_{ \pm}$ some arbitrarily small neighbourhood of $\pm m$, and as shown in [Wro12] such state will satisfy the Hadamard condition, it will however not be gaugeinvariant.

Sufficient conditions for the second case to hold are given in [Gér12]. This includes for instance the case when $\Sigma=\mathbb{R}^{d}, A_{i} \equiv 0, V \in \mathscr{D}\left(\mathbb{R}^{d}\right) \cap L^{d}\left(\mathbb{R}^{d}\right)$ and $m<\|V\|_{\infty}<$ $\sqrt{2} m$.

The proof of the Hadamard condition is similar to the Dirac case, except that we have additionally to show that the term with $\Pi_{+}$does not contribute to the wave front set. In [Wro12] we used a simpler definition which did not involve the $\Pi_{+}$term, it did however not yield a gauge-invariant state.

Proposition 5.3.1. The sesquilinear form $\lambda(\cdot, \cdot)=[\cdot \mid P \cdot]$ defined by (5.3.1) is the two-point function of a pure, gauge-invariant, quasi-free Hadamard state $\omega$.

Proof. Let us define $\Lambda^{( \pm)}:=\tau^{*} \tilde{\Lambda}^{( \pm)}, S_{0}:=\tau^{*} \tilde{S}_{0}$, where

$$
\begin{aligned}
\left\langle\tilde{\Lambda}^{( \pm)}, f \otimes \bar{u} \otimes v\right\rangle & =-\left[u \mid\left(\mathcal{F}^{-1} f\right)(B) \mathbb{1}_{\mathcal{J}_{ \pm}}(B) v\right], \\
\left\langle\tilde{S}_{0}, f \otimes \bar{u} \otimes v\right\rangle & =\mathrm{i}\left[u \mid\left(\mathcal{F}^{-1} f\right)(B)\left(\mathbb{1}^{\mathbb{C} \backslash \mathbb{R}}(B)+\mathbb{1}_{\mathbb{R} \backslash\left(\mathcal{J}_{-} \cup \mathcal{J}_{+}\right)}(B)\right) v\right],
\end{aligned}
$$

for $f \in \mathscr{D}(\mathbb{R}), u, v \in \mathscr{D}(\Sigma, \mathscr{V})$.

We show that the assumptions of Theorem 4.3.3 are satisfied. By (5.1.5) and Proposition 5.2.10 we have $\mathrm{i} S=\Lambda^{(-)}-\Lambda^{(+)}+\mathrm{i} S_{0}$ and by construction, $\Lambda_{\mathcal{J}}^{( \pm)}, S_{0}$ are bi-solutions for $D$. Since the range of $\mathbb{1}^{\mathbb{C} \backslash \mathbb{R}}(B)+\mathbb{1}_{\mathbb{R} \backslash\left(\mathcal{J}_{-} \cup \mathcal{J}_{+}\right)}(B)$ is spanned by a finite set $\left\{\varphi_{i}\right\}$ of eigenvectors of $B, \tilde{S}_{0}$ restricted to $t=$ const is proportional to the integral kernel of

$$
\left.\sum_{i} \mathrm{e}^{\mathrm{i} \lambda{ }_{i} t} \mid \varphi_{i}\right)\left(\varphi_{i} \mid\right.
$$

where $\lambda_{i}$ are the respective eigenvalues. We see that $\tilde{S}_{0}$ is smooth in the time variable, i.e. $\operatorname{WF}\left(\tilde{S}_{0}\right) \subset \Gamma^{0}$. Since it is a bi-solution for $D, \operatorname{WF}\left(\tilde{S}_{0}\right) \cap \Gamma^{0}=\emptyset$. This yields $\mathrm{WF}\left(S_{0}\right)=\emptyset$, hence $\mathrm{i} S=\Lambda_{\mathcal{J}}^{(+)}-\Lambda_{\mathcal{J}}^{(-)} \bmod C^{\infty}$.

To prove that $\tilde{\Lambda}^{(+)}$is tempered in the time direction we have to show $\tilde{\Lambda}_{\mathcal{J}}^{(+)}$: $\mathscr{S}(\mathbb{R}) \otimes \mathscr{D}(\Sigma, \mathscr{V}) \otimes \mathscr{D}(\Sigma, \mathscr{V}) \rightarrow \mathbb{C}$ is continuous. By Schwarz inequality and smooth 
functional calculus, there exists $m \in \mathbb{N}$ s.t.

$$
\begin{aligned}
\left|\left\langle\tilde{\Lambda}^{(+)}, f \otimes \bar{u} \otimes v\right\rangle\right|=\left|\left[u \mid\left(\mathcal{F}^{-1} f\right)(B) \mathbb{1}_{\mathcal{J}_{+}}(B) v\right]\right| & =\left|\left(u \mid J\left(\mathcal{F}^{-1} f\right)(B) \mathbb{1}_{\mathcal{J}_{+}}(B) v\right)_{\mathcal{K}}\right| \\
& \leq\|u\|_{\mathcal{K}}\left\|\left(\mathcal{F}^{-1} f\right)(B)\right\|\left\|J \mathbb{1}_{\mathcal{J}_{+}}(B)\right\|\|v\|_{\mathcal{K}} \\
& \leq C_{B}\|u\|_{\mathcal{K}}\left\|\mathcal{F}^{-1} f\right\|_{m}\left\|J \mathbb{1}_{\mathcal{J}_{+}}(B)\right\|\|v\|_{\mathcal{K}} .
\end{aligned}
$$

Convergence of $f$ to 0 in $\mathscr{S}(\mathbb{R})$ implies $\mathcal{F}^{-1} f \rightarrow 0$ in $\mathscr{S}(\mathbb{R})$ and consequently $\left\|\mathcal{F}^{-1} f\right\|_{m} \rightarrow 0$. Furthermore, convergence of $u$ (resp. $v$ ) to 0 in $\mathscr{D}(\Sigma, \mathscr{V})$ implies $\|u\|_{\mathcal{K}} \rightarrow 0$ (resp. $\|v\|_{\mathcal{K}}$ ). Indeed, this follows from the inequality

$$
\|u\|_{\mathcal{K}}^{2}=\left\|\epsilon^{1 / 2} u_{0}\right\|^{2}+\left\|\epsilon^{-1 / 2} u_{1}\right\|^{2} \leq\left\|\epsilon^{-3 / 2}\right\|^{2}\left\|\epsilon^{2} u_{0}\right\|^{2}+\left\|\epsilon^{-1 / 2}\right\|^{2}\left\|u_{1}\right\|^{2} .
$$

Now, $u_{0}, u_{1} \rightarrow 0$ in $\mathscr{D}(\Sigma, \mathscr{V})$ implies $\epsilon^{2} u_{0}, u_{1} \rightarrow 0$ in $\mathscr{D}(\Sigma, \mathscr{V})$ and consequently $\left\|\epsilon^{2} u_{0}\right\|$, $\left\|u_{1}\right\| \rightarrow 0$. The reasoning for $\tilde{\Lambda}^{(-)}$is analogous.

Finally, to see that $\mathcal{F}_{0} \tilde{\Lambda}^{( \pm)}$have the required support properties, observe that the distributions $\mathcal{F}_{0} \tilde{\Lambda}^{(+)}, \mathcal{F}_{0} \tilde{\Lambda}^{(-)}$are uniquely determined by their value on simple tensors:

$$
\left\langle\mathcal{F}_{0} \tilde{\Lambda}^{( \pm)}, f \otimes \bar{u} \otimes v\right\rangle=-\mathrm{i}\left[u \mid f(B) \mathbb{1}_{\mathcal{J}_{ \pm}}(B) v\right],
$$

By 3. of Proposition 5.2.8, (5.3.2) vanishes for each $f \in \mathscr{S}(\mathbb{R})$ with $\operatorname{supp} f \cap \operatorname{sp}_{\mathrm{cr}}(B)=$ $\emptyset$ and $\operatorname{supp} f \cap \overline{\mathcal{J}_{ \pm}}=\emptyset$. Therefore,

$$
\operatorname{supp}\left(\mathcal{F}_{0} \tilde{\Lambda}^{( \pm)}\right) \subset\left(\operatorname{sp}_{\mathrm{cr}}(B) \cup \overline{\mathcal{J}_{ \pm}}\right) \times \Sigma^{\times 2},
$$

which by boundedness of the set $\operatorname{sp}_{\mathrm{cr}}(B)$ finishes the proof.

The physical interpretation of the Hadamard state $\omega$ constructed in the overcritical case $(2)$ can be made clearer by viewing $\mathcal{K}$ as a direct sum of two spaces: the range of $\mathbb{1}_{\mathcal{J}_{+}}(B)+\mathbb{1}_{\mathcal{J}_{-}}(B)$ and the finite dimensional space spanned by the remaining eigenvectors of $B$. In fact, $\lambda$ restricted to the first component yields a ground state in the usual sense. The restriction to the second component is not a ground state, but this poses no problem from the point of view of the Hadamard condition.

On the other hand, it does not seem possible to decompose the physical system in a similar way in the more singular situation (3), and the problem of constructing gauge-invariant Hadamard states with properties close to a ground state remains open. 



\section{Part 2}

Strength of singularities: Renormalisation and interacting fields 



\section{CHAPTER 6}

\section{Renormalisation and scaling degree}

In local perturbative QFT, the $S$-matrix is defined as a formal power series in the coupling constant. In the formulation proposed by Brunetti and Fredenhagen [BF00], the theory is determined by the choice of a Hadamard state $\omega$ of the non-interacting theory and a Lagrangian density $\mathscr{L}(x)$ which accounts for the interaction. It is a distribution with values in operators on the Hilbert space $\mathcal{H}_{\omega}$, obtained from the GNS construction for the state $\omega$. Additionally, an adiabatic cutoff $\eta \in \mathscr{D}(M)$ has to be introduced to avoid infrared problems. The ansatz for the $S$-matrix in terms of a Dyson series

$$
S(\eta):=\mathbf{1}+\sum_{m=1}^{\infty} \frac{\mathrm{i}^{m}}{m !} \int_{M \times m} T\left(\mathscr{L}\left(x_{1}\right) \otimes \cdots \otimes \mathscr{L}\left(x_{m}\right)\right) \eta\left(x_{1}\right) \ldots \eta\left(x_{m}\right) d \Omega_{g}^{m},
$$

can be solved by the method of Epstein and Glaser [EG73], after imposing a set of defining axioms on the time-ordered product $T$. These axioms, proposed and worked out in [SR50, BP57, BS59], can be extended to curved spacetime in a very natural way. The main difficulty is that most terms in the series (6.0.1) involve ambiguous products of distributions, that cannot be defined just by making use of sufficient conditions on their wave front sets. The rigorous definition of the time-ordered products, the study of resulting ambiguities and the implementation of global symmetries are the central questions in renormalisation in the Epstein and Glaser approach.

In what follows, we review the main elements of the construction and introduce a mathematical notion due to Steinmann [Ste71], which is particularly useful in the problem of extending singular distributions - the scaling degree. The material that we present here serves as an introduction to the results of the next chapters and can be found in the references [EG73, Sch95, BF00, HR02, SVW02, DF03], it can be skipped by the expert reader familiar with the toolset of extensions of distributions.

\subsection{The algebra of Wick polynomials}

6.1.1. Operator-valued distributions. To formulate the result of Brunetti and Fredenhagen in an effective way, it is useful to make the following definitions. If $\mathcal{H}$ is a Hilbert space and $\mathcal{D}$ some subspace (not necessarily closed), then we denote $\mathcal{D}(\mathcal{H})$ the algebra of all operators $A$ (not necessarily closed), such that

$$
\operatorname{Dom} A \subset \mathcal{D}, \quad \operatorname{Ran} A \subset \mathcal{D} .
$$

We endow additionally $\mathcal{D}(\mathcal{H})$ with the family of seminorms $p_{\Psi, \Phi}(A):=|(\Psi \mid A \Phi)|$, $\Psi, \Phi \in \mathcal{D}$, turning it into a locally convex topological space. 
In this section we will deal with operator-valued distribution $\phi \in \mathscr{D}^{\prime}(M, \mathcal{D}(\mathcal{H}))$ and by writing $\phi(f):=\langle\phi, f\rangle$ for $f \in \mathscr{D}(M)$, we will mean the element of $\mathcal{D}(\mathcal{H})$ obtained via the pairing $\langle\cdot, \cdot\rangle$ between operator-valued distributions and ordinary test densities.

We will need a generalization of the wave-front set adapted to the case of operatorvalued distributions. First, observe that the original definition of the wave front set of $u \in \mathscr{D}^{\prime}(M)$ (cf. Definition 3.2.1) refers to estimates on $|\widehat{\varphi u}|$ for $\varphi \in \mathscr{D}(M)$. This extends directly to distributions with values in elements of a Banach space, by replacing $|\widehat{\varphi u}|$ with $\|\widehat{\varphi u}\|$, cf. [SVW02]. The properties of the wave front set defined this way are to a large extent analogous to what we have already seen in the case of ordinary distributions, for instance one has essentially the same criteria for the existence of restrictions to submanifolds, products of distributions, etc. (cf. Theorem 3.2.2). Finally, if $\phi \in \mathscr{D}^{\prime}(M, \mathcal{D}(\mathcal{H}))$, we set

$$
\mathrm{WF}(\phi):=\bigcup_{\Psi \in \mathcal{D}} \mathrm{WF}(\phi \Psi) .
$$

The above definition is somewhat artificial, as it refers to some subset $\mathcal{D}$ of $\mathcal{H}$ which does not need to coincide with the domain of $\phi$, but it is completely satisfactory for the applications in the next section.

6.1.2. Wick monomials. In what follows we briefly recall the construction of Wick polynomials due to Brunetti and Fredenhagen. Our intention is to make the connection between the Hadamard connection and renormalisation, and for that purpose it is sufficient to consider the case of neutral spin-0 fields. The letter $P$ will denote the Klein-Gordon operator on a globally hyperbolic spacetime $(M, g)$.

Assume we are given a Hadamard state $\omega$ and let $\left(\mathcal{H}_{\omega}, \pi_{\omega}, \Omega_{\omega}\right)$ be the associated GNS representation. To the corresponding field operators we associate an operatorvalued distribution $\phi(x)$ in the obvious way. Let $\mathcal{H}_{\omega}^{\infty}$ be the joint domain of field operators (cf. (1.3.5)). Conforming to the point-splitting strategy, we first define Wick monomials, such as : $\phi\left(x_{1}\right) \phi\left(x_{2}\right)$ :, by substracting the singular part using the twofunction $\Lambda^{(+)}(x, y)$, and later on we will take the limit of coinciding points. Formulas for higher Wick monomials involve products such as $\Lambda^{(+)}\left(x_{1}, x_{2}\right) \Lambda^{(+)}\left(x_{2}, x_{3}\right)$ - this is where the Hadamard condition enters, it implies that these products are well-defined indeed. To avoid the use of long combinatorial expressions, it is useful to express the definition of Wick monomials in the language of formal derivatives (we refer the reader to [BF00, HR02] for precise definitions and more detailed discussion). All these observations are used in the formulation of the following theorem.

Theorem 6.1.1 ([BF00]). There exists a dense subspace $\mathcal{D}_{\omega} \subset \mathcal{H}_{\omega}$, contained in $\mathcal{H}_{\omega}^{\infty}$ and such that for $m \in \mathbb{N}$, the formal functional derivative

$$
\left.\frac{\delta^{m}}{\delta f\left(x_{1}\right) \ldots f\left(x_{m}\right)}\left[\exp \left\{\frac{1}{2} \iint f(x) \Lambda^{(+)}(x, y) f(y) d \Omega_{g}^{2}+\mathrm{i} \int \phi(x) f(x) d \Omega_{g}\right\}\right]\right|_{f=0}
$$

yields a well defined distribution on $M^{\times m}$ with values in $\mathcal{D}_{\omega}\left(\mathcal{H}_{\omega}\right)$, denoted : $\phi^{\otimes m}$ : 
Moreover,

$$
\mathrm{WF}\left(: \phi^{\otimes m}:\right) \subset\left\{\left(x_{1}, \xi_{1}, \ldots, x_{m}, \xi_{m}\right): \xi_{i} \in V_{x_{i}-}^{*}, i=1, \ldots, m\right\} .
$$

The special form of the wave front set (6.1.1) allows to define Wick powers $: \phi^{m}$ : as distributions on $M$ with values in $\mathcal{D}_{\omega}\left(\mathcal{H}_{\omega}\right)$, by taking the restriction of : $\phi^{\otimes m}$ : to the diagonal

$$
\Delta_{m}:=\left\{\left(x_{1}, \ldots, x_{m}\right) \in M^{\times m}: x_{1}=\ldots=x_{m}\right\} .
$$

Equivalently, this can be done by setting

$$
: \phi^{m}(f)::=\left\langle: \phi^{\otimes m}:, f \delta_{\Delta_{m}}\right\rangle, \quad f \in \mathscr{D}(M)
$$

where the bracket $\langle\cdot, \cdot\rangle$ on the right hand side denotes the pairing between distributions $u, v$ s.t. $\mathrm{WF}(u) \cap-\mathrm{WF}(v)=\emptyset$ (cf. Section 3.2), and $\delta_{\Delta_{m}}$ is the Dirac measure supported at $\Delta_{m}$. More generally, the right hand side makes sense if $f \delta_{\Delta_{m}}$ is replaced by any distribution $u$ which belongs to the set

$$
\mathcal{W}_{m}:=\left\{u \in \mathscr{E}^{\prime}\left(M^{\times m}\right): u \text { symmetric, } \operatorname{WF}(u) \cap\left(\bigcup_{x} V_{x+}^{*} \cup V_{x-}^{*}\right)=\emptyset\right\} .
$$

This observation is used in [DF01a] to obtain a particularly convenient form of Wick's theorem. Namely, one considers the direct sum $\mathcal{W}:=\bigoplus_{m=0}^{\infty} \mathcal{W}_{m}, \mathcal{W}_{0}:=\mathbb{C}$, and endow it with an associative product

$$
(u \cdot v)_{m}:=\sum_{m+2 k=l+i} u_{i} \otimes_{k} v_{l}, \quad u=\left(u_{0}, u_{1} \ldots\right), v=\left(v_{0}, v_{1}, \ldots\right) \in \mathcal{W}
$$

where

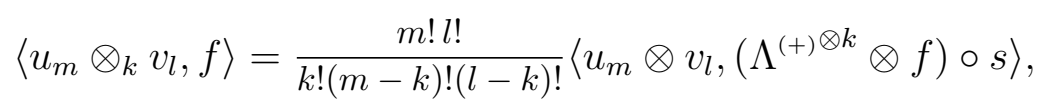

for symmetric $f \in \mathscr{D}\left(M^{m+l-2 k}\right)$ and $m \geq k, l \geq k$, and $s: M^{\times(m+l)} \rightarrow M^{\times(m+l)}$ is given by $s\left(x_{1}, \ldots, x_{m}, y_{1}, \ldots, y_{l}\right)=\left(x_{1}, y_{1}, \ldots, x_{k}, y_{k}, x_{k+1}, \ldots, x_{m}, y_{k+1}, \ldots, y_{l}\right)$. The above definitions are tailored in such way that the algebra product in $\mathcal{W}$ encodes the combinatorics of Wick's theorem, which takes the following form.

Theorem 6.1.2 ([DF01a, DF01b]). The map

$$
\mathcal{W}_{m} \ni u \mapsto: \phi^{\otimes m}(u): \in \mathcal{D}_{\omega}\left(\mathcal{H}_{\omega}\right)
$$

extends by linearity to an algebra homomorphism $\tau: \mathcal{W} \rightarrow \mathcal{D}_{\omega}\left(\mathcal{H}_{\omega}\right)$ with kernel

$$
\operatorname{Ker} \tau=\left\{\sum_{k}\left(P^{\mathrm{t}}\right)^{\otimes k} u_{k}: u_{k} \in \mathcal{W}_{k}\right\}
$$

The algebra $\mathcal{W}$ is called the algebra of Wick polynomials. It also contains elements that correspond to Wick products of derivatives of fields. For instance, the expression $: \phi \nabla_{\mu} \phi$ : evaluated on $f \in \mathscr{D}(M)$ is defined as the image of the symmetrization of $\left(f \otimes \nabla_{\mu}^{\mathrm{t}}\right) \delta_{\Delta_{2}}$ via the homomorphism $\tau$ (cf. [Mor03] for a detailed discussion).

The corresponding definitions for charge fields are more complicated, since both the distributions $\Lambda^{( \pm)}$need to enter. The algebra of Wick polynomials was studied in this case by Marecki [Mar03], see also [Zah13].

Remark 6.1.3. It is possible to obtain similar results for Wick powers defined by renormalising with the Hadamard parametrix instead of the two-point function of a 
Hadamard state [HW01]. This approach, not pursued here, has the advantage that it eventually produces interacting fields whose dependence on the underlying spacetime is local and covariant with respect to isometric embeddings which preserve causal relations.

Let us mention that a recent result of Sanders states that $: \phi^{2}(f)$ : is essentially selfadjoint on the domain $\bigcup_{m} \phi\left(f_{1}\right) \ldots \phi\left(f_{m}\right) \Omega_{\omega}$, provided that $f$ is the sum of squares of test functions [San12a]. It is however unknown whether essential self-adjointness holds on the 'microlocal domain of smoothness' $\mathcal{D}_{\omega}$ from Theorem 6.1.1 introduced by Brunetti and Fredenhagen.

In what follows we will work in a more abstract setting, which is possible after identifying $\mathcal{W}$ with an algebra that has the interpretation of an algebra of classical fields [DF03].

To this end, one defines off-shell classical fields, denoted $\varphi_{x}$, as evaluation functionals on $\mathscr{E}\left(\mathbb{R}^{n}\right)$ (the classical configuration space), namely $\left(\varphi_{x}\right)(h):=h(x)$ for $h \in$ $\mathscr{E}\left(\mathbb{R}^{n}\right)$. The derivatives of classical off-shell fields are defined by $\left(\partial^{\alpha} \varphi_{x}\right)(h):=\partial^{\alpha} h(x)$. The algebra of off-shell fields $\mathcal{P}$ is the commutative algebra generated by elements of the form $\partial^{\alpha} \varphi_{x}$ with respect to the pointwise product, i.e. $\left(\partial^{\alpha_{1}} \varphi_{x} \partial^{\alpha_{2}} \varphi_{x}\right)(h):=$ $\partial^{\alpha_{1}} h(x) \partial^{\alpha_{2}} h(x)$. The algebra of classical on-shell fields is the quotient algebra $\mathcal{P}_{0}:=$ $\mathcal{P} / \mathcal{J}$, where $\mathcal{J}$ is the ideal

$$
\mathcal{J}:=\left\{\sum_{\alpha \in \mathbb{N}_{0}} B_{\alpha} \partial^{\alpha}\left(\square+m^{2}\right) \varphi: B_{\alpha} \in \mathcal{P}\right\} .
$$

We denote by $\pi: \mathcal{P} \rightarrow \mathcal{P}_{0}$ the canonical surjection, i.e. $\pi(B)=B+\mathcal{J}$ for $B \in \mathcal{P}$. One can easily see that it is a homomorphism of algebras. The derivatives of on-shell fields are defined for $A \in \mathcal{P}_{0}$ by $\partial^{\mu} A:=\pi \partial^{\mu} B$, where $B$ is an arbitrary element of $\mathcal{P}$ such that $\pi(B)=A$. One can check that this does not depend on the choice of $B$ and that one has $\left(\square+m^{2}\right) \pi \varphi=0$ in $\mathcal{P}_{0}$ for any $\varphi \in \mathcal{P}$ - this is the onshell property. In consequence, it follows from Theorem 6.1.2 that there exists an isomorphism $\mathcal{W} \rightarrow \mathcal{P}_{0}$.

6.1.3. On-shell time-ordered products. Time-ordered products $T$ of order $k$ are defined as maps from $\mathcal{W}^{\otimes k}$ to operator-valued distributions. Using the isomorphism $\mathcal{W} \rightarrow \mathcal{P}_{0}$, one can equally well consider a map $T_{\text {on }}$ from $\mathcal{P}_{0}$ to operator-valued distributions. The axioms defining $T_{\text {on }}$ and its inductive construction are the basic components of the Epstein and Glaser approach to renormalisation and are a subject covered exhaustively by the literature [EG73, Sch95, DF01a, DF03]. The crucial assumption is the factorization property - it states that for any $m, n \in \mathbb{N}$, if $x_{i} \cap J^{-}\left(y_{j}\right)=\emptyset$ for all $i=1, \ldots, m$ and $j=1, \ldots, k$, then the time-ordered product

$$
T_{\text {on }}\left(\mathscr{L}\left(x_{1}\right) \otimes \cdots \otimes \mathscr{L}\left(x_{m}\right) \otimes \mathscr{L}\left(y_{1}\right) \otimes \cdots \otimes \mathscr{L}\left(y_{k}\right)\right)
$$

decomposes as

$$
T_{\text {on }}\left(\mathscr{L}\left(x_{1}\right) \otimes \cdots \otimes \mathscr{L}\left(x_{m}\right)\right) T_{\text {on }}\left(\mathscr{L}\left(y_{1}\right) \otimes \cdots \otimes \mathscr{L}\left(y_{k}\right)\right) .
$$


This property is obtained upon requiring causality of the $S$-matrix, i.e.,

$$
\eta_{1}, \eta_{2} \in \mathscr{D}(M), \operatorname{supp} \eta_{1} \cap \operatorname{supp}\left(J^{-}\left(\eta_{2}\right)\right)=\emptyset \Rightarrow S\left(\eta_{1}+\eta_{2}\right)=S\left(\eta_{1}\right) S\left(\eta_{2}\right)
$$

in the sense of products of formal power series. With the exception of problematic points lying on the total diagonal $\Delta_{m}$, the factorization property allows to express time-ordered products of order $m$ in terms of time-ordered products of lower order. Using the well-known Wick expansion theorem (cf. [BF00, Thm. 2.4]), it is additionally possible to reduce the problem to ordinary distributions, therefore one ends up with the task of extending distributions defined on $M^{\times m} \backslash \Delta_{m}$ to $M^{\times m}$. In the special case of a flat spacetime and vanishing external potentials, translation-invariance can be then used to reduce the problem to extension of distributions originally given on $\dot{\mathbb{R}}^{n}:=\mathbb{R}^{n} \backslash\{0\}$ to distributions on $\mathbb{R}^{n}$. Otherwise, an expansion in curvature terms [HW02], and in covariant quantities derived from the potentials [Zah13], can be applied to reduce the problem to a flat one.

\subsection{Scaling degree}

As a preparation to the discussion of extension of distributions, in what follows we recall the definition of Steinman's scaling degree of a distribution on $\dot{\mathbb{R}}^{n}$ or $\mathbb{R}^{n}$ [Ste71]. Although this notion plays nowadays a key role in renormalisation [BF00], it does not seem to have entered the mathematical literature as such. Note, however, that similar estimates have been used in e.g. [Est98]. Moreover, Steinman's scaling degree for distributions on $\mathbb{R}^{n}$ is closely related to two-microcal spaces, see for instance [Mey98] and references therein, and is also a degenerate case of Weinstein's degree of a distribution [Wei78].

Consider the natural action of the dilation group $\mathbb{R}_{>0}$ on $\mathscr{D}\left(\mathbb{R}^{n}\right)$ and its dual on $\mathscr{D}^{\prime}\left(\mathbb{R}^{n}\right)$, i.e. for a distribution $u \in \mathscr{D}^{\prime}\left(\mathbb{R}^{n}\right)$ and $\lambda>0$ set

$$
\left\langle u_{\lambda}, \varphi\right\rangle:=\lambda^{-n}\left\langle u, \varphi\left(\lambda^{-1} \cdot\right)\right\rangle, \quad \varphi \in \mathscr{D}\left(\mathbb{R}^{n}\right) .
$$

Definition 6.2.1. The scaling degree of $u \in \mathscr{D}^{\prime}\left(\mathbb{R}^{n}\right)$, denoted $\mathrm{sd} u$, is the infimum over all $\omega \in \mathbb{R}$ s.t. $\lim _{\lambda \searrow_{0}} \lambda^{\omega} u_{\lambda}=0$ in $\mathscr{D}^{\prime}\left(\mathbb{R}^{n}\right)$. The degree of divergence of $u$ is $\operatorname{deg} u:=\operatorname{sd} u-n$.

The degree of divergence of a distribution $u \in \mathscr{D}^{\prime}\left(\dot{\mathbb{R}}^{n}\right)$, denoted also $\operatorname{deg} u$, is defined analogously (one simply replaces $\mathscr{D}\left(\mathbb{R}^{n}\right)$ by $\mathscr{D}\left(\dot{\mathbb{R}}^{n}\right)$ and $\mathscr{D}^{\prime}\left(\mathbb{R}^{n}\right)$ by $\mathscr{D}^{\prime}\left(\dot{\mathbb{R}}^{n}\right)$ in the above definition). The difference is that it is possible here that the limit $\lim _{\lambda \searrow 0} \lambda^{\omega} u_{\lambda}$ does not exist for any $\omega \in \mathbb{R}$. In this case we write deg $u=\infty$.

We will use the degree of divergence rather than the scaling degree, as it is more convenient in our framework. As a basic example, observe that the derivatives of the $\delta$ distribution $\delta^{(\alpha)}$ on $\mathbb{R}^{n}$ have degree of divergence $|\alpha|$. A function in $\mathscr{E}\left(\mathbb{R}^{n}\right)$, considered as an element of $\mathscr{D}^{\prime}\left(\mathbb{R}^{n}\right)$, has degree of divergence at most $-n$. A distribution which is homogeneous of degree $a \in \mathbb{C}$ on $\mathbb{R}^{n}$ (resp. $\dot{\mathbb{R}}^{n}$ ) has degree of divergence $-\operatorname{Re} a-n$ in $\mathscr{D}^{\prime}\left(\mathbb{R}^{n}\right)$ (resp. $\mathscr{D}^{\prime}\left(\dot{\mathbb{R}}^{n}\right)$ ).

Let us briefly recall the basic properties of the degree of divergence, which were 
proved $^{1}$ in [BF00, Lemma 5.1].

LEMmA 6.2.2. Let $u \in \mathscr{D}^{\prime}\left(\mathbb{R}^{n}\right)$ and assume $\operatorname{deg} u<\infty$. Then:

(1) For $\alpha \in \mathbb{N}^{n}, \operatorname{deg}\left(\partial^{\alpha} u\right) \leq \operatorname{deg} u+|\alpha|$.

(2) For $\alpha \in \mathbb{N}^{n}, \operatorname{deg}\left(x^{\alpha} u\right) \leq \operatorname{deg} u-|\alpha|$.

(3) Let $f \in \mathscr{E}\left(\mathbb{R}^{n}\right)$ and assume $f^{(\alpha)}(0)=0$ for $|\alpha| \leq k-1$, then $\operatorname{deg}(f u) \leq$ $\operatorname{deg} u-k$.

(4) Let $v \in \mathscr{D}^{\prime}\left(\mathbb{R}^{k}\right)$ then $\operatorname{sd}(u \otimes v) \leq \operatorname{sd} u+\operatorname{sd} v$.

6.2.1. Extension of distributions. Let us recall the basic ingredients of the construction of extensions of distributions. Essentially, we follow [BF00], but for later purposes, we make systematic use of the following spaces of distributions. Denote by $\mathscr{D}^{\prime}(\{0\})$ the space of distributions supported at $\{0\}$. For $r \geq 0$, let $\mathscr{D}^{\prime}(\{0\})_{\leq r}$ be the subspace of $\mathscr{D}^{\prime}(\{0\})$ given by those of maximal degree $r$,

$$
\mathscr{D}^{\prime}(\{0\})_{\leq r}=\operatorname{span}\left\{v \in \mathscr{D}^{\prime}(\{0\}): \operatorname{deg} v \leq r\right\}=\operatorname{span}\left\{\delta^{(\alpha)} \in \mathscr{D}^{\prime}\left(\mathbb{R}^{n}\right):|\alpha| \leq r\right\} .
$$

On the other hand, consider the space of all test functions vanishing up to order $r$ at $x=0$ :

$$
\mathscr{D}_{r}\left(\mathbb{R}^{n}\right):=\left\{\varphi \in \mathscr{D}\left(\mathbb{R}^{n}\right):\left(\partial_{x}^{\alpha} \varphi\right)(0)=0 \quad \forall \alpha \in \mathbb{N}_{0}^{n},|\alpha| \leq r\right\} .
$$

It will be convenient to generalize this definition in the following way. Let $\mathcal{K}$ be a finite dimensional subspace of $\mathscr{D}^{\prime}(\{0\})$. Set

$$
\mathscr{D}_{\mathcal{K}}\left(\mathbb{R}^{n}\right):=\left\{\varphi \in \mathscr{D}\left(\mathbb{R}^{n}\right):\langle v, \varphi\rangle=0 \quad \forall v \in \mathcal{K}\right\} .
$$

Clearly, $\mathscr{D}_{r}\left(\mathbb{R}^{n}\right)$ equals $\mathscr{D}_{\mathcal{K}}\left(\mathbb{R}^{n}\right)$ with $\mathcal{K}=\mathscr{D}^{\prime}(\{0\})_{\leq r}$. Observe that the scaling degree of a distribution in $\mathscr{D}_{\mathcal{K}}^{\prime}\left(\mathbb{R}^{n}\right)$ can be defined in an analogous way to the scaling degree in $\mathscr{D}^{\prime}\left(\mathbb{R}^{n}\right)$. We now restate Theorem 5.2 from [BF00] as follows:

Proposition 6.2.3. Let $u \in \mathscr{D}^{\prime}\left(\dot{\mathbb{R}}^{n}\right)$ have degree of divergence $r:=\operatorname{deg} u<\infty$. Then it admits a unique extension $\tilde{u} \in \mathscr{D}_{r}^{\prime}\left(\mathbb{R}^{n}\right)$ with the same degree of divergence $r$, given by

$$
\langle\tilde{u}, \varphi\rangle:=\lim _{\rho \rightarrow \infty}\left\langle u,\left(1-\vartheta_{\rho}\right) \varphi\right\rangle, \quad \varphi \in \mathscr{D}_{r}\left(\mathbb{R}^{n}\right)
$$

where $\vartheta_{\rho}(x):=\vartheta\left(2^{\rho} x\right)$ and $\vartheta$ is an arbitrary function in $\mathscr{D}\left(\mathbb{R}^{n}\right)$ such that $\vartheta=1$ in a neighbourhood of the origin.

It now remains to find elements of $\mathscr{D}^{\prime}\left(\mathbb{R}^{n}\right)$ which correspond to the extension $\tilde{u} \in \mathscr{D}_{r}^{\prime}\left(\mathbb{R}^{n}\right)$. Following the ideas of $[\mathrm{BF} 00]$, we do so by considering projections ${ }^{2}$ $W^{\mathrm{t}}: \mathscr{D}\left(\mathbb{R}^{n}\right) \rightarrow \mathscr{D}_{r}\left(\mathbb{R}^{n}\right)$, and applying their transpose $W: \mathscr{D}_{r}^{\prime}\left(\mathbb{R}^{n}\right) \rightarrow \mathscr{D}^{\prime}\left(\mathbb{R}^{n}\right)$ to $\tilde{u} \in \mathscr{D}_{r}^{\prime}\left(\mathbb{R}^{n}\right)$.

To this end, let us first state two lemmas which are slight generalizations of results found in [DF04] where they were stated for $\mathcal{K}=\mathscr{D}^{\prime}(\{0\})_{\leq r}$.

\footnotetext{
${ }^{1}$ Strictly speaking, the third claim is considered there only for $k=0$, but the case $k \geq 1$ follows immediately by noting that under the assumptions, such $f$ equals $x_{1}^{k} \ldots x_{n}^{k} g$ for some $g \in \mathscr{E}\left(\mathbb{R}^{n}\right)$ and then using 2.

${ }^{2}$ This means that $W^{\mathrm{t}}: \mathscr{D}\left(\mathbb{R}^{n}\right) \mapsto \mathscr{D}_{r}\left(\mathbb{R}^{n}\right)$ is continuous and $\left(W^{\mathrm{t}}\right)^{2}=W^{\mathrm{t}}$.
} 
Lemma 6.2.4. Let $\mathcal{K}$ be a finite dimensional subspace of $\mathscr{D}^{\prime}(\{0\})$, let $\left\{v_{i}\right\}_{i \in \mathcal{I}}$ be a basis of $\mathcal{K}$, and assume $\left\{\psi_{i}\right\}_{i \in \mathcal{I}}$ is a family $\psi_{i} \in \mathscr{D}\left(\mathbb{R}^{n}\right)$ s.t. $\left\langle v_{i}, \psi_{j}\right\rangle=\delta_{i j}$. Then

$$
W^{\mathrm{t}} \varphi=\varphi-\sum_{i \in \mathcal{I}}\left\langle v_{i}, \varphi\right\rangle \psi_{i}
$$

defines a projection $W^{\mathrm{t}}: \mathscr{D}\left(\mathbb{R}^{n}\right) \rightarrow \mathscr{D}_{\mathcal{K}}\left(\mathbb{R}^{n}\right)$. Conversely, if $W^{\mathrm{t}}: \mathscr{D}\left(\mathbb{R}^{n}\right) \rightarrow \mathscr{D}_{\mathcal{K}}\left(\mathbb{R}^{n}\right)$ is a projection, there is a family $\left\{\psi_{i}\right\}_{i \in \mathcal{I}}$ with the above properties.

Lemma 6.2.5. Let $u \in \mathscr{D}_{\mathcal{K}}^{\prime}\left(\mathbb{R}^{n}\right)$ and let $W^{\mathrm{t}}: \mathscr{D}\left(\mathbb{R}^{n}\right) \rightarrow \mathscr{D}_{\mathcal{K}}\left(\mathbb{R}^{n}\right)$ be a projection. Then $\langle W u, \varphi\rangle=\langle u, \varphi\rangle$ for all $\varphi \in \mathscr{D}_{\mathcal{K}}\left(\mathbb{R}^{n}\right)$ and $\operatorname{deg} W u=\operatorname{deg} u$.

Taking into account that $\operatorname{deg} \delta^{(\alpha)}=|\alpha|$, we find the following important result on the existence of extensions with the same degree of divergence.

Corollary 6.2.6. ([BF00]) Let $u \in \mathscr{D}^{\prime}\left(\dot{\mathbb{R}}^{n}\right)$ be a distribution with $r:=\operatorname{deg} u<\infty$. Then there is an extension $\dot{u} \in \mathscr{D}^{\prime}\left(\mathbb{R}^{n}\right)$ of $u$ with $\operatorname{deg} \dot{u}=\operatorname{deg} u$. Each such extension can be written as $\dot{u}=W \tilde{u}$, where $\tilde{u}$ is the unique extension of $u$ in $\mathscr{D}_{r}^{\prime}\left(\mathbb{R}^{n}\right)$ and $W^{\mathrm{t}}: \mathscr{D}\left(\mathbb{R}^{n}\right) \rightarrow \mathscr{D}_{r}\left(\mathbb{R}^{n}\right)$ is a projection. Moreover, two arbitrary extensions with the above properties differ by an element of $\mathscr{D}^{\prime}(\{0\})_{\leq r}$.

While each extension of $u$ with the same degree of divergence can be constructed as above by using a projection $W^{\mathrm{t}}$, it can sometimes be more convenient to use some other operator $V^{\mathrm{t}}$ which maps $\mathscr{D}\left(\mathbb{R}^{n}\right)$ to $\mathscr{D}_{r}\left(\mathbb{R}^{n}\right)$ and check whether $V \tilde{u}$ is an extension of $u$ with the correct degree of divergence. In fact, this approach will prove to be more convenient in directly constructing on-shell extensions (cf. Proposition 7.2.7). 



\section{CHAPTER 7}

\section{On-shell extension of distributions}

As we outlined in the previous chapter, the renormalisation problem in the Epstein and Glaser approach is reformulated as a problem of extending distributions defined on $\mathbb{R}^{n} \backslash\{0\}=: \dot{\mathbb{R}}^{n}$ to distributions on $\mathbb{R}^{n}$. By construction, any two extensions can differ by a distribution supported in 0 , and one way to constrain this ambiguity is to require that the extension should have the same scaling degree as the original distribution. In QFT, the ambiguity is further constrained by imposing physically motivated renormalisation conditions. Such conditions include the requirement that if $u \in \mathscr{D}^{\prime}\left(\dot{\mathbb{R}}^{n}\right)$ respects a global symmetry, e.g. it is invariant under the Lorentz group or a global gauge group, then the same should hold for its extension to $\mathbb{R}^{n}$. Another such condition, which turned out to be essential in renormalisation on both flat and on curved space-time [HW02], is the requirement that if $u$ is homogeneous, its extension should be homogeneous as well, or at least it should behave as much like a homogeneous distribution as possible (a property which can be properly formulated in terms of almost homogeneous distributions).

A problem which at first sight seems to be unrelated to such renormalisation conditions occurs in the construction of on-shell time ordered products involving higher derivatives of quantum fields. Roughly speaking, one wishes to extend to $\mathbb{R}^{n}$ an expression in $\mathscr{D}^{\prime}\left(\mathbb{R}^{n}\right)$ involving derivatives of the Feynman propagator and Heaviside theta functions such that the extension satisfies the (free) equation of motion. The possibility of finding such an extension can be rephrased using the relation between on-shell time-ordered products (ordinarily used in quantum field theory) and off-shell products, the latter of which have proved to be better suited for a theoretical study of Epstein-Glaser renormalisation [DF03, DF04].

The main idea in the approach proposed in [BW12] is that all these problems can be formulated and solved in a unified framework, by restating them in terms of the existence of extensions which solve a set of (differential) equations. More precisely, we state the following extension problem:

Problem Let $\left\{Q^{i}\right\}_{i=1}^{k}$ be a family of differential operators on $\mathbb{R}^{n}$ with smooth coefficients, and let $u$ be a distribution in $\mathscr{D}^{\prime}\left(\dot{\mathbb{R}}^{n}\right)$ that satisfies

$$
Q^{i} u=0 \quad \text { on } \dot{\mathbb{R}}^{n} \quad(i=1, \ldots, k) .
$$

Find $\dot{u} \in \mathscr{D}^{\prime}\left(\mathbb{R}^{n}\right)$ such that $\dot{u}=u$ on $\dot{\mathbb{R}}^{n}$ and $Q^{i} \dot{u}=0$ on $\mathbb{R}^{n}(i=1, \ldots, k)$. If such extensions $\dot{u}$ exist, we call them on-shell extensions (w.r.t. $\left\{Q^{i}\right\}_{i=1}^{k}$ ).

Indeed, invariance of a distribution under the action of a connected Lie group is equivalent to it being a solution of its infinitesimal generators. (Almost) homogeneity 
is described using (powers of) the operator $\sum_{i} x_{i} \partial_{i}-a$. In the construction of onshell time-ordered products, the differential operator of interest is the Klein-Gordon operator $\left(\square+m^{2}\right)$. To include discrete symmetries, we will consider a more general class of operators later.

On the mathematical side, the 'on-shell extension' problem we consider is closely related to the so-called Bochner's extension problem, an issue which we explain in the text.

One advantage of our reformulation is the following. The various constructions and prescriptions to implement renormalisation conditions proposed so far in e.g. [Sch95, Pra99, Gra03, LG03, DF04, HW02], see also [DG12], are each limited to a particular type of symmetry. Therefore, the simultaneous implementation of a number of different conditions requires cumbersome proofs of compatibility. Our framework on the other hand, allows for a compact formulation of e.g. sufficient conditions on the existence of extensions subject to different renormalisation conditions, such as Lorentz invariance considered together with almost homogeneity and (eventually) parity. Moreover, it exhibits a new feature of Epstein-Glaser renormalisation: a renormalisation condition corresponding to a (differential) operator $Q$ can be imposed by applying a linear map to a generic extension (which is a solution of $Q$ on $\dot{\mathbb{R}}^{n}$ ). This statement extends to the case of several renormalisation conditions.

Concerning the relation between on-shell and off-shell time-ordered products, we would like to point out that it was given in [DF03, DF04] in terms of a recurrence relation for which an explicit solution was found in [BD08]. In our framework we find a more compact formula, which contrary to that given in [BD08] does not contain unnatural combinatorial factors. Instead, the coefficients which appear in our formula are simply eigenvalues of certain finite-dimensional operators directly related to the Klein-Gordon operator.

This chapter is organized as follows. Section 7.1 contains the main ideas and results. First, in subsection 7.1.1, we introduce the notion of operators of essential order $m$ on $\mathscr{D}^{\prime}\left(\mathbb{R}^{n}\right)$. Such operators generalize differential operators and will enable us to treat discrete symmetries. In subsection 7.1 .2 we equip the finite dimensional spaces of distributions of given maximal order supported at the origin with a scalar product. The restrictions of operators $Q: \mathscr{D}^{\prime}\left(\mathbb{R}^{n}\right) \rightarrow \mathscr{D}^{\prime}\left(\mathbb{R}^{n}\right)$ to these spaces play an important role in subsection 3.2, especially for Theorem 7.2.2. This theorem provides a solution to the extension problem with respect to an operator $Q$ of arbitrary essential order in the sense that it lists different statements which are equivalent to the existence of on-shell extensions, and provides a candidate for such an extension. This candidate can be calculated explicitly and only requires one to find the eigenvalues of a finite dimensional matrix. Special cases, examples and generalizations are then discussed. Of particular interest is Theorem 7.2.8 which states sufficient, and easyto-check conditions that ensure the existence of on-shell extensions w.r.t. an operator of essential order 0 . We then explain how the extension problem with respect to a finite number of operators is solved. Section 8.1 is devoted to the construction of 
on-shell time-ordered products involving higher derivatives of the fields. We clarify how the relation between the on-shell and the off-shell formalism can be formulated and understood in our framework.

\subsection{Operators of finite essential order}

7.1.1. Essential order. Our aim is to implement symmetries, i.e. we will ask our extensions to satisfy a set of given equations. In order to include discrete symmetries, we consider more general operators from $\mathscr{D}^{\prime}\left(\mathbb{R}^{n}\right)$ to $\mathscr{D}^{\prime}\left(\mathbb{R}^{n}\right)$ rather than just differential operators.

Definition 7.1.1. We say that $Q: \mathscr{D}^{\prime}\left(\mathbb{R}^{n}\right) \rightarrow \mathscr{D}^{\prime}\left(\mathbb{R}^{n}\right)$ is an operator of essential order $q$ if

(1) $Q$ is the transpose of a linear operator $Q^{\mathrm{t}}: \mathscr{E}\left(\mathbb{R}^{n}\right) \rightarrow \mathscr{E}\left(\mathbb{R}^{n}\right)$ which continuously maps $\mathscr{D}\left(\dot{\mathbb{R}}^{n}\right)$ and $\mathscr{D}\left(\mathbb{R}^{n}\right)$ to themselves;

(2) $q \in \mathbb{N}_{0}$ is the lowest number such that $\operatorname{deg} Q u \leq \operatorname{deg} u+q$ for all $u \in \mathscr{D}^{\prime}\left(\mathbb{R}^{n}\right)$.

Basic examples for such operators are of course differential operators, for which the essential degree was already considered in [Nik07]: a differential operator of order $m$ has essential order smaller or equal $m$. More precisely, a differential operator $Q=\sum_{|\alpha| \leq m} a_{\alpha}(x) \partial^{\alpha}$ has essential order $q$, where $q$ is the smallest possible nonnegative number s.t. $\left(\partial^{\beta} a_{\alpha}\right)(0)=0$ for $|\beta| \leq|\alpha|-q-1$. In particular, $Q$ has essential order 0 if $\left(\partial^{\beta} a_{\alpha}\right)(0)=0$ for $|\beta| \leq|\alpha|-1$.

Let us list some basic properties of operators of essential order $q$.

Lemma 7.1.2. Let $Q: \mathscr{D}^{\prime}\left(\mathbb{R}^{n}\right) \rightarrow \mathscr{D}^{\prime}\left(\mathbb{R}^{n}\right)$ be an operator of essential order $q$. Then

(1) $Q$ is continuous in the $\mathscr{D}^{\prime}\left(\mathbb{R}^{n}\right)$ topology;

(2) $Q$ maps $\mathscr{D}^{\prime}\left(\dot{\mathbb{R}}^{n}\right)$ and $\mathscr{D}^{\prime}(\{0\})$ to themselves.

(3) Let $\mathcal{K}_{1}$ be a linear subspace of $\mathscr{D}^{\prime}(\{0\})$. Then $Q^{\mathrm{t}}$ maps $\mathscr{D}_{\mathcal{K}_{1}}\left(\mathbb{R}^{n}\right)$ to $\mathscr{D}_{\mathcal{K}_{2}}\left(\mathbb{R}^{n}\right)$, where

$$
\mathcal{K}_{2}=\left\{v \in \mathscr{D}^{\prime}(\{0\}) \mid Q v \in \mathcal{K}_{1}\right\} .
$$

In particular, $Q^{\mathrm{t}}$ maps $\mathscr{D}\left(\mathbb{R}^{n}\right)$ to $\mathscr{D}_{\mathcal{K}}\left(\mathbb{R}^{n}\right)$, where $\mathcal{K}=\operatorname{Ker}\left(\left.Q\right|_{\mathscr{D}^{\prime}(\{0\})}\right)$.

Proof.

(1) By definition of the weak topology.

(2) The first assertion is obvious. For the second one it suffices to notice that for any $v \in \mathscr{D}^{\prime}(\{0\})$ the expression $\langle Q v, \varphi\rangle$ only depends on the restriction of $\varphi$ to an arbitrary small neighbourhood of 0 .

(3) Let $\varphi \in \mathscr{D}_{\mathcal{K}_{1}}\left(\mathbb{R}^{n}\right)$, then $Q^{\mathrm{t}} \varphi \in \mathscr{D}_{\mathcal{K}_{2}}\left(\mathbb{R}^{n}\right)$, since for any $v \in \mathcal{K}_{2}$, i.e. $v \in$ $\mathscr{D}^{\prime}(\{0\})$ such that $Q v \in \mathcal{K}_{1}$, we have $\left\langle v, Q^{\mathrm{t}} \varphi\right\rangle=\langle Q v, \varphi\rangle=0$.

The following two lemmas give examples of operators of essential degree 0 , for which we usually reserve the symbol $R$.

LEmma 7.1.3. Let $R$ be an infinitesimal generator of a Lie group $G$ acting on $\mathbb{R}^{n}$ such that 0 is a fixed point. Then $R$ is an operator of essential degree 0 . 
Proof. Indeed, $R=\sum_{i=1}^{n} \xi^{i}(x) \partial_{i}$ where $\xi^{i}(0)=0$ (as follows from, i.e., [Olv95, Ex. $2.68])$.

Lemma 7.1.4. Let $\Phi: \mathbb{R}^{n} \rightarrow \mathbb{R}^{n}$ be a $C^{\infty}$ diffeomorphism s.t. $\Phi(0)=0$. Then the operator given by $R u:=u-\Phi^{*} u$ for $u \in \mathscr{D}^{\prime}\left(\mathbb{R}^{n}\right)$ is an operator of essential degree 0 .

Proof. Since $\Phi(0)=0$, we find that $\operatorname{supp} \varphi \cap\{0\}=\emptyset \operatorname{implies} \operatorname{supp}\left(\Phi^{*} \varphi\right) \cap\{0\}=\emptyset$. Let us now check that $\operatorname{sd}\left(\Phi^{*} u\right) \leq \operatorname{sd} u$ for all $u \in \mathscr{D}^{\prime}\left(\mathbb{R}^{n}\right)$. Indeed, $\tau^{-\omega}\langle u, \varphi(\tau x)\rangle \rightarrow$ 0 for all $\varphi \in \mathscr{D}\left(\mathbb{R}^{n}\right)$ implies that $\tau^{-\omega}\left\langle u,\left(\Phi_{*} \phi\right)(\tau x)\right\rangle \rightarrow 0$ for all $\phi \in \mathscr{D}\left(\mathbb{R}^{n}\right)$, so $\tau^{-\omega}\left\langle\Phi^{*} u, \phi(\tau x)\right\rangle \rightarrow 0$.

These two cases are of particular importance in our applications. To see this, first recall that a distribution $u \in \mathscr{D}^{\prime}\left(\mathbb{R}^{n}\right)$ is invariant under the induced action of a connected Lie group $G$ acting on $\mathbb{R}^{n}$ if and only if $R^{i} u=0$ for all the infinitesimal generators $R^{i}$ of $G$. Now, if 0 is a fixed point of the action of $G$ on $\mathbb{R}^{n}$, then by the first of the above lemmas, the infinitesimal generators are of essential order 0 .

Similarly, discrete symmetries entail operators of essential degree 0 , as they are of the form discussed in the second lemma. For instance, even distributions are in the kernel of the operator $R^{+} u:=u-u(-\cdot)$, and odd ones in that of $R^{-} u:=u+u(-\cdot)$.

7.1.2. Spaces of distributions supported at the origin. Recall that $\mathscr{D}^{\prime}(\{0\})_{\leq r}$ denotes the finite dimensional vector space spanned by derivatives of the delta distribution up to order $r$. It it will turn out to be very useful to equip it with a scalar product. To this end, for $r \geq 0$ define the maps

$$
\begin{aligned}
& \mathcal{S}_{r}: \mathscr{D}^{\prime}(\{0\})_{\leq r} \rightarrow \mathscr{E}\left(\mathbb{R}^{n}\right), \quad \mathcal{S}_{r} v:=\sum_{|\alpha| \leq r} \frac{x^{\alpha}}{\alpha !}\left\langle v, x^{\alpha}\right\rangle, v \in \mathscr{D}^{\prime}(\{0\})_{\leq r} \\
& \mathcal{T}_{r}: \mathscr{E}\left(\mathbb{R}^{n}\right) \rightarrow \mathscr{D}^{\prime}(\{0\})_{\leq r}, \quad \mathcal{T}_{r} f:=\sum_{|\alpha| \leq r} \frac{\delta^{(\alpha)}}{\alpha !}\left\langle\delta^{(\alpha)}, f\right\rangle, f \in \mathscr{E}\left(\mathbb{R}^{n}\right) .
\end{aligned}
$$

One can easily check that $\mathcal{T}_{r} \mathcal{S}_{r}=$ id on $\mathscr{D}^{\prime}(\{0\})_{\leq r}$ and $\mathcal{S}_{r} \mathcal{T}_{r}=$ id on the space of polynomials of degree $\leq r$. Now set

$$
(v \mid w)_{r}:=\left\langle\bar{v}, \mathcal{S}_{r} w\right\rangle=\sum_{|\alpha| \leq r} \frac{1}{\alpha !}\left\langle\bar{v}, x^{\alpha}\right\rangle\left\langle w, x^{\alpha}\right\rangle=\left\langle w, \mathcal{S}_{r} \bar{v}\right\rangle, \quad v, w \in \mathscr{D}^{\prime}(\{0\})_{\leq r}
$$

where the bar denotes ordinary complex conjugation. Writing elements $v, w$ of $\mathscr{D}^{\prime}(\{0\})_{\leq r}$ as linear combinations $v=\sum_{|\alpha| \leq r} v_{\alpha} \delta^{(\alpha)}, w=\sum_{|\alpha| \leq r} w_{\alpha} \delta^{(\alpha)}$, with $v_{\alpha}, w_{\alpha} \in \mathbb{C}$, we have

$$
(v \mid w)_{r}=\left\langle\sum_{|\alpha| \leq r} \bar{v}_{\alpha} \delta^{(\alpha)}, \sum_{|\beta| \leq r}(-1)^{\beta} w_{\beta} x^{\beta}\right\rangle=\sum_{|\alpha| \leq r} \alpha ! \bar{v}_{\alpha} w_{\alpha},
$$

therefore it is evident that $(\cdot \mid \cdot)_{r}$ is a scalar product on $\mathscr{D}^{\prime}(\{0\})_{\leq r}$.

Let $Q: \mathscr{D}^{\prime}\left(\mathbb{R}^{n}\right) \rightarrow \mathscr{D}^{\prime}\left(\mathbb{R}^{n}\right)$ be an operator of essential order $q$. We denote

$$
\left.Q\right|_{r}: \mathscr{D}^{\prime}(\{0\})_{\leq r} \rightarrow \mathscr{D}^{\prime}(\{0\})_{\leq r+q}
$$

the restriction of $Q$ to $\mathscr{D}^{\prime}(\{0\})_{\leq r}$, understood as an operator from $\mathscr{D}^{\prime}(\{0\})_{\leq r}$ to $\mathscr{D}^{\prime}(\{0\})_{\leq r+q}$. Let us stress that this definition depends on the essential order of $Q$. The next lemma characterizes the adjoint of $\left.Q\right|_{r}$. 
Lemma 7.1.5. Let $Q$ be an operator of essential order $q$. Then the adjoint of $\left.Q\right|_{r}$ : $\mathscr{D}^{\prime}(\{0\})_{\leq r} \rightarrow \mathscr{D}^{\prime}(\{0\})_{\leq r+q}$ is

$$
\left(\left.Q\right|_{r}\right)^{*}=\mathcal{T}_{r} \bar{Q}^{\mathrm{t}} \mathcal{S}_{r+q}: \mathscr{D}^{\prime}(\{0\})_{\leq r+q} \rightarrow \mathscr{D}^{\prime}(\{0\})_{\leq r} .
$$

Proof. For all $v \in \mathscr{D}^{\prime}(\{0\})_{\leq r+q}$ and $w \in \mathscr{D}^{\prime}(\{0\})_{\leq r}$ one has

$$
\begin{aligned}
(v \mid Q w)_{r+q} & =\left\langle\bar{v}, \mathcal{S}_{r+q} Q w\right\rangle=\left\langle Q w, \mathcal{S}_{r+q} \bar{v}\right\rangle=\left\langle w, Q^{\mathrm{t}} \mathcal{S}_{r+q} \bar{v}\right\rangle \\
& =\left\langle\mathcal{T}_{r} \mathcal{S}_{r} w, Q^{\mathrm{t}} \mathcal{S}_{r+q} \bar{v}\right\rangle=\left\langle\mathcal{S}_{r} w, \mathcal{T}_{r} Q^{\mathrm{t}} \mathcal{S}_{r+q} \bar{v}\right\rangle=\left(\mathcal{T}_{r} \bar{Q}^{\mathrm{t}} \mathcal{S}_{r} v \mid w\right)_{r},
\end{aligned}
$$

so (7.1.1) follows.

We will need for certain results an assumption which guarantees that $\left(\left.Q\right|_{r}\right)^{*}$ and $\left(\left.Q\right|_{r^{\prime}}\right)^{*}$ are in a sense compatible for $r \neq r^{\prime}$.

DEFINITION 7.1.6. We say that an operator $Q$ of essential order $q$ is essentially homogeneous if $Q^{\mathrm{t}}$ maps polynomials of order $\leq r+q$ to elements of

$$
\left\{f \in C^{\infty}\left(\mathbb{R}^{n}\right): f^{(\alpha)}(0)=0,|\alpha|>r\right\} .
$$

Lemma 7.1.7. Let $Q$ be essentially homogeneous. Then for all $r \in \mathbb{N}_{0}$ and $s \geq r$ the restriction of the operator $\left(\left.Q\right|_{s}\right)^{*}$ to $\mathscr{D}^{\prime}(\{0\})_{\leq r+q}$ equals $\left(\left.Q\right|_{r}\right)^{*}$.

Proof. We use Lemma 7.1.5 and observe that $\mathcal{S}_{s+q}$ coincides with $\mathcal{S}_{r+q}$ on $\mathscr{D}^{\prime}(\{0\})_{\leq r+q}$ and $\mathcal{T}_{s}$ coincides with $\mathcal{T}_{r}$ on the space (7.1.2). Therefore, $\mathcal{T}_{s} \bar{Q}^{\mathrm{t}} \mathcal{S}_{s+q}$ restricted to $\mathscr{D}^{\prime}(\{0\})_{\leq r+q}$ equals $\mathcal{T}_{r} \bar{Q}^{\mathrm{t}} \mathcal{S}_{r+q}$.

A sufficient condition for an operator $Q$ to be essentially homogeneous is that $Q^{\mathrm{t}}$ maps polynomials of degree $r+q$ to polynomials of degree $r$.

EXAmple 2. The operators $\partial_{x}, x \partial_{x}, x \partial_{x}+\cos x$ are essentially homogeneous, but $\partial_{x}+m$ is not unless $m=0$.

\subsection{On-shell extension — single operator case}

Let us specify the problem we already outlined in the introduction, first for the case when only one operator is considered.

Problem. Let $Q: \mathscr{D}^{\prime}\left(\mathbb{R}^{n}\right) \rightarrow \mathscr{D}^{\prime}\left(\mathbb{R}^{n}\right)$ be an operator of essential order $q$. Let $u \in \mathscr{D}^{\prime}\left(\dot{\mathbb{R}}^{n}\right)$ have degree of divergence $r:=\operatorname{deg} u<\infty$ and assume

$$
Q u=0 \quad \text { on } \dot{\mathbb{R}}^{n} \text {. }
$$

Find $\ddot{u} \in \mathscr{D}^{\prime}\left(\mathbb{R}^{n}\right)$ such that $\ddot{u}=u$ on $\dot{\mathbb{R}}^{n}$ and $Q \ddot{u}=0$ on $\mathbb{R}^{n}$. If such extension(s) exist, we call them on-shell extensions w.r.t. $Q$.

The next lemma is an easy observation, which will however play a key role in our approach to the problem of finding on-shell extensions.

Lemma 7.2.1. Let $Q: \mathscr{D}^{\prime}\left(\mathbb{R}^{n}\right) \rightarrow \mathscr{D}^{\prime}\left(\mathbb{R}^{n}\right)$ be an operator of essential order $q$. Let $u \in \mathscr{D}^{\prime}\left(\dot{\mathbb{R}}^{n}\right)$ have $r:=\operatorname{deg} u<\infty$ and assume

$$
Q u=0 \quad \text { on } \dot{\mathbb{R}}^{n} \text {. }
$$


Then the following statements are equivalent:

a) $u$ has an on-shell extension $\ddot{u} \in \mathscr{D}^{\prime}\left(\mathbb{R}^{n}\right)$ s.t. $\operatorname{deg} \ddot{u}=r$,

b) $Q \dot{u} \in \operatorname{Ran}\left(\left.Q\right|_{r}\right)$ for all extensions $\dot{u} \in \mathscr{D}^{\prime}\left(\mathbb{R}^{n}\right)$ of $u$ with $\operatorname{deg} \dot{u}=r$;

c) $Q \dot{u} \in \operatorname{Ran}\left(\left.Q\right|_{r}\right)$ for some extension $\dot{u} \in \mathscr{D}^{\prime}\left(\mathbb{R}^{n}\right)$ of $u$ with $\operatorname{deg} \dot{u}=r$;

Proof. a) $\Rightarrow$ b): Assume $Q \ddot{u}=0$. Since for all extensions $\dot{u}$ of $u$ with $\operatorname{deg} \dot{u}=\operatorname{deg} u=r$ we have $\dot{u}=\ddot{u}+v$ for some $v \in \mathscr{D}^{\prime}(\{0\})_{\leq r}$, it follows that $Q \dot{u}=Q(\ddot{u}+v)=Q v \in$ $\operatorname{Ran}\left(\left.Q\right|_{r}\right)$.

b) $\Rightarrow \mathrm{c})$ : is obvious.

c) $\Rightarrow$ a): Assume $Q \dot{u}=Q v$ for some $v \in \mathscr{D}^{\prime}(\{0\})_{\leq r}$. Then $\ddot{u}:=\dot{u}-v$ satisfies $Q \ddot{u}=0$.

Let us now focus on the following problem - in the case when $u \in \mathscr{D}^{\prime}\left(\dot{\mathbb{R}}^{n}\right)$ has on-shell extensions w.r.t. $Q$, how can they be constructed? From Lemma 7.2.1 we know that if $\dot{u}$ is a generic extension with degree of divergence $r$, then $\dot{u}$ is onshell modulo an element of $\operatorname{Ran}\left(\left.Q\right|_{r}\right)$. If the problem was purely finite-dimensional, we could get rid of the remainder in $\operatorname{Ran}\left(\left.Q\right|_{r}\right)$ using the orthogonal projection to $\left(\operatorname{Ran}\left(\left.Q\right|_{r}\right)\right)^{\perp}$. Observe that such projection can be expressed as a polynomial in the operator $\left(\left.Q\right|_{r}\right)^{*}\left(\left.Q\right|_{r}\right)$. As we show below, it suffices to consider such polynomials in $\left(\left.Q\right|_{r}\right)^{*} Q$ instead, to get an operator with the desired properties which is well-defined on distributions $\dot{u} \in \mathscr{D}^{\prime}\left(\mathbb{R}^{n}\right)$.

Theorem 7.2.2. Let $Q: \mathscr{D}^{\prime}\left(\mathbb{R}^{n}\right) \rightarrow \mathscr{D}^{\prime}\left(\mathbb{R}^{n}\right)$ be an operator of essential order $q$. Denote

$$
\operatorname{Sol}_{\text {ext }}(Q)=\left\{v \in \mathscr{D}^{\prime}\left(\mathbb{R}^{n}\right): Q v=0 \text { on } \dot{\mathbb{R}}^{n}, \operatorname{deg} v \neq \infty\right\}
$$

Let $\mathcal{R}: \operatorname{Sol}_{\text {ext }}(Q) \rightarrow \operatorname{Sol}_{\text {ext }}(Q)$ be defined by

$$
\mathcal{R} v:=p_{r}\left(\left(\left.Q\right|_{r}\right)^{*} Q\right) v, \quad r=\operatorname{deg} v,
$$

where $p_{r}$ is the polynomial $p_{r}(z)=\prod_{\lambda}(1-z / \lambda)$, the product being taken over all nonzero $\lambda \in \operatorname{sp}\left(\left(\left.Q\right|_{r}\right)\left(\left.Q\right|_{r}\right)^{*}\right)$. Then for all $v \in \operatorname{Sol}_{\text {ext }}(Q)$,

(1) $\mathcal{R} v=v$ on $\dot{\mathbb{R}}^{n}$

(2) $\operatorname{deg} \mathcal{R} v \leq \operatorname{deg} v$

(3) $\operatorname{deg} Q \mathcal{R} v \leq \operatorname{deg} Q w$ for all $w \in \operatorname{Sol}_{\text {ext }}(Q)$ with $w=v$ on $\dot{\mathbb{R}}^{n}$ and $\operatorname{deg} w=$ $\operatorname{deg} v$.

In particular, $\mathcal{R} v$ is an on-shell extension of $v$ w.r.t. $Q$ iff such on-shell extension exists.

Proof. Fix $v \in \operatorname{Sol}_{\text {ext }}(Q)$ and denote $r=\operatorname{deg} v$. Since $Q v \in \mathscr{D}^{\prime}(\{0\})_{\leq r+q}$ and $\left(\left.Q\right|_{r}\right)^{*}$ maps $\mathscr{D}^{\prime}(\{0\})_{\leq r+q}$ to $\mathscr{D}^{\prime}(\{0\})_{\leq r}$, the distribution $\left(\left.Q\right|_{r}\right)^{*} Q v \in \mathscr{D}^{\prime}(\{0\})_{\leq r}$ is well defined. Since $p_{r}(0)=1$, we also have

$$
\mathcal{R} v-v=\left(p_{r}-1\right)\left(\left(\left.Q\right|_{r}\right)^{*} Q\right) v \in \mathscr{D}^{\prime}(\{0\})_{\leq r} .
$$

This proves (1) and (2). To prove (3), we compute

$$
Q \mathcal{R} v=Q p_{r}\left(\left(\left.Q\right|_{r}\right)^{*} Q\right) v=p_{r}\left(Q\left(\left.Q\right|_{r}\right)^{*}\right) Q v=p_{r}\left(\left(\left.Q\right|_{r}\right)\left(\left.Q\right|_{r}\right)^{*}\right) Q v
$$


Observe that $p_{r}\left(\left(\left.Q\right|_{r}\right)\left(\left.Q\right|_{r}\right)^{*}\right)$ is the orthogonal projection to $\left(\left.\operatorname{Ran} Q\right|_{r}\right)^{\perp}$, hence $Q \mathcal{R} v \in$ $\left(\left.\operatorname{Ran} Q\right|_{r}\right)^{\perp}$. For any $w \in \mathscr{D}^{\prime}\left(\mathbb{R}^{n}\right)$ with $w=v$ on $\dot{\mathbb{R}}^{n}$ and $\operatorname{deg} w=r$, we can decompose

$$
Q w=Q \mathcal{R} v+\left.Q(w-\mathcal{R} v) \in\left(\left.\operatorname{Ran} Q\right|_{r}\right)^{\perp} \oplus \operatorname{Ran} Q\right|_{r},
$$

therefore $\operatorname{deg} Q w \geq \operatorname{deg} Q \mathcal{R} v$.

Given a distribution $u \in \mathscr{D}^{\prime}\left(\dot{\mathbb{R}}^{n}\right)$ with degree of divergence $r$ s.t. $Q u=0$ and an arbitrary extension of $\dot{u}$ with $\operatorname{deg} \dot{u}=r$, the distribution $\mathcal{R} \dot{u}$ is a candidate for an on-shell extension of $u$. If no on-shell extension exists, then $\mathcal{R} \dot{u}$ is 'as close to being an on-shell extension as possible'. To calculate it explicitly, one only has to find the eigenvalues of the finite-dimensional matrix $\left(\left.Q\right|_{r}\right)\left(\left.Q\right|_{r}\right)^{*}$.

There is another remarkable feature of the map $\mathcal{R}$. It turns out that under the additional assumptions that $Q$ is essentially homogeneous (cf. Definition 7.1.6), $\mathcal{R}$ is linear on $\operatorname{Sol}_{\text {ext }}(Q)$. This statement is obvious when one speaks only of distributions with a fixed degree of divergence $r$. However, it is not at all evident if one considers distributions with different degrees of divergence, for both the definition of $p_{r}$ and $\left(\left.Q\right|_{r}\right)^{*}$ in (7.2.1) depend on $r$.

Proposition 7.2.3. Let $Q$ be an operator of essential order $q$ and assume it is essentially homogeneous. Then the map $\mathcal{R}: \operatorname{Sol}_{\text {ext }}(Q) \rightarrow \operatorname{Sol}_{\text {ext }}(Q)$ from Theorem 7.2.2 is linear.

Proof. Let $v_{1}, v_{2} \in \operatorname{Sol}_{\text {ext }}(Q)$ and denote $r_{1}=\operatorname{deg} v_{1}, r_{2}=\operatorname{deg} v_{2}$ and $r=\operatorname{deg}\left(v_{1}+\right.$ $\left.v_{2}\right) \leq \max \left\{r_{1}, r_{2}\right\}$. To prove that

$$
p_{r}\left(\left(\left.Q\right|_{r}\right)^{*} Q\right)\left(v_{1}+v_{2}\right)=p_{r_{1}}\left(\left(\left.Q\right|_{r_{1}}\right)^{*} Q\right) v_{1}+p_{r_{2}}\left(\left(\left.Q\right|_{r_{2}}\right)^{*} Q\right) v_{2}
$$

it suffices to show that

$$
0=\left[p_{r^{\prime}}\left(\left(\left.Q\right|_{r^{\prime}}\right)^{*} Q\right)-p_{r}\left(\left(\left.Q\right|_{r}\right)^{*} Q\right)\right] v
$$

for all $r^{\prime} \geq r$ and $v \in \operatorname{Sol}_{\text {ext }}(Q)$ with $\operatorname{deg} v=r$. One has

$$
\left[p_{r^{\prime}}\left(\left(\left.Q\right|_{r^{\prime}}\right)^{*} Q\right)-p_{r}\left(\left(\left.Q\right|_{r}\right)^{*} Q\right)\right] v=\left(p_{r^{\prime}}-p_{r}\right)\left(\left(\left.Q\right|_{r}\right)^{*} Q\right) v
$$

because $\left(\left.Q\right|_{r^{\prime}}\right)^{*}$ restricted to $\mathscr{D}^{\prime}(\{0\})_{\leq r+q}$ equals $\left(\left.Q\right|_{r}\right)^{*}$ by Lemma 7.1.7. Since $\left(p_{r^{\prime}}-\right.$ $\left.p_{r}\right)(0)=0$, the expression $\left(p_{r^{\prime}}-p_{r}\right)\left(\left(\left.Q\right|_{r}\right)^{*} Q\right)$ is the sum of elements of the form $\left(\left(\left.Q\right|_{r}\right)^{*} Q\right)^{k}$. For $Q \dot{u} \in \mathscr{D}^{\prime}(\{0\})_{\leq r+q}$, we conclude $\left(p_{r^{\prime}}-p_{r}\right)\left(\left(\left.Q\right|_{r}\right)^{*} Q\right) \dot{u} \in \operatorname{Ran}\left(\left.Q\right|_{r}\right)^{*}$. On the other hand, observe that

$$
Q\left(p_{r^{\prime}}-p_{r}\right)\left(\left(\left.Q\right|_{r}\right)^{*} Q\right) \dot{u}=\left(p_{r^{\prime}}-p_{r}\right)\left(\left(\left.Q\right|_{r}\right)\left(\left.Q\right|_{r}\right)^{*}\right) Q \dot{u} .
$$

By Lemma 7.1.7, $\left(p_{r^{\prime}}-p_{r}\right)\left(\left(\left.Q\right|_{r}\right)\left(\left.Q\right|_{r}\right)^{*}\right)$ restricted to $\mathscr{D}^{\prime}(\{0\})_{\leq r+q}$ is the difference of two orthogonals projections on the same subspace, hence vanishes on $Q \dot{u} \in \mathscr{D}^{\prime}(\{0\})_{\leq r+q}$. This means that $\left(p_{r^{\prime}}-p_{r}\right)\left(\left(\left.Q\right|_{r}\right)^{*} Q\right) \dot{u}$ belongs to $\operatorname{Ker}\left(\left.Q\right|_{r}\right)=$ $\left(\operatorname{Ran}\left(\left.Q\right|_{r}\right)^{*}\right)^{\perp}$ and therefore vanishes.

The problem of finding on-shell extensions can be thought as a variant of the following Bochner's extension problem:

Problem Let $u \in \mathscr{D}^{\prime}\left(\mathbb{R}^{n}\right)$ and assume $P u=0$ on $\dot{\mathbb{R}}^{n}$. Does $P u=0$ hold on $\mathbb{R}^{n}$ ? 
If $P u=0$ on $\mathbb{R}^{n}$, one says that $u$ has a removable singularity for $P$ at 0 , see [RS10] and references therein for a collection of results on that subject. Observe that the assumption $P u=0$ on $\dot{\mathbb{R}}^{n}$ implies that $P u$ is supported in 0 , and a computation of the degree of divergence gives $P u \in \mathscr{D}^{\prime}(\{0\})_{\leq r+m}$, where $r=\operatorname{deg} u$ and $m$ is the essential order of $P$. In particular, we obtain that $P u=0$ on $\mathbb{R}^{n}$ if $\operatorname{deg} u<-m$. This gives a useful sufficient condition for removable singularities, which can be rephrased in terms of commonly used function spaces such as $L^{p}$ or Sobolev spaces, more suitable for various applications outside quantum field theory. On the other hand, the theorems proved by us are particularly useful in the more singular case when $\operatorname{deg} u \geq-m$ as a distribution on $\dot{\mathbb{R}}^{n}$.

7.2.1. Operators of essential order 0. As we have seen, operators of essential order 0 are of special interest in the applications. Moreover, they map $\mathscr{D}^{\prime}(\{0\})_{\leq r}$ to itself, so one can study the natural subclasses consisting of self-adjoint or normal operators and use their special properties in the analysis. Also, for an operator of essential order 0 , we will give sufficient conditions for the existence of on-shell extensions which are easy to check.

In what follows, $R: \mathscr{D}^{\prime}\left(\mathbb{R}^{n}\right) \rightarrow \mathscr{D}^{\prime}\left(\mathbb{R}^{n}\right)$ is always an operator of essential order 0 .

If $\left.R\right|_{r}$ is normal, then $\operatorname{Ker}\left(\left.R\right|_{r}\right)=\operatorname{Ker}\left(\left.R\right|_{r}\right)^{*}=\operatorname{Ker}\left(\left.R\right|_{r}\right)^{k}$ for $k \in \mathbb{N}_{0}$. This fact is used in the proof of the next proposition, which provides additional information when no on-shell extension exists for $R$ (or more generally $R^{k}$ ). This will for instance be the case for homogeneous distributions of degree $a$ s.t. $-a \in \mathbb{N}_{0}+n$, which in general do not have homogeneous extensions to $\mathbb{R}^{n}$.

Proposition 7.2.4. Let $R$ be of essential order 0. Let $u \in \mathscr{D}^{\prime}\left(\dot{\mathbb{R}}^{n}\right)$ have degree of divergence $r<\infty$ and suppose

$$
R^{k} u=0 \quad \text { on } \dot{\mathbb{R}}^{n}
$$

for some $k \in \mathbb{N}_{0}$. If $\left.R\right|_{r}$ is normal, there exists an extension $\ddot{u} \in \mathscr{D}^{\prime}\left(\mathbb{R}^{n}\right)$ of $u$ with $\operatorname{deg} \ddot{u}=\operatorname{deg} u$ such that

$$
R^{k+1} \ddot{u}=0 \quad \text { on } \mathbb{R}^{n} .
$$

More precisely, one can take $\ddot{u}=p_{r}\left(\left(\left.R^{k}\right|_{r}\right)^{*} R^{k}\right) \dot{u}$, where $\dot{u} \in \mathscr{D}^{\prime}\left(\mathbb{R}^{n}\right)$ is an arbitrary extension of $u$ with $\operatorname{deg} \dot{u}=\operatorname{deg} u$ and $p_{r}(z)=\prod_{\lambda}(1-z / \lambda)$, the product being taken over all nonzero $\lambda \in \operatorname{sp}\left(\left(\left.R^{k}\right|_{r}\right)\left(\left.R^{k}\right|_{r}\right)^{*}\right)$.

Proof. One has

$$
R^{k+1} \ddot{u}=R^{k+1} p_{r}\left(\left(\left.R^{k}\right|_{r}\right)^{*} R^{k}\right) \dot{u}=R p_{r}\left(R^{k}\left(\left.R^{k}\right|_{r}\right)^{*}\right) R^{k} \dot{u}=R p_{r}\left(\left(\left.R^{k}\right|_{r}\right)\left(\left.R^{k}\right|_{r}\right)^{*}\right) R^{k} \dot{u} .
$$

This vanishes because $R^{k} \dot{u} \in \mathscr{D}^{\prime}(\{0\})_{\leq r}$ and $p_{r}\left(\left(\left.R^{k}\right|_{r}\right)\left(\left.R^{k}\right|_{r}\right)^{*}\right)$ is the orthogonal projection to $\operatorname{Ker}\left(\left.R^{k}\right|_{r}\right)^{*}$, which by normality of $\left.R\right|_{r}$ equals $\operatorname{Ker}\left(\left.R\right|_{r}\right)$.

An analogue of Proposition 7.2.3 is available: 
Proposition 7.2.5. Let $R$ be of essential order 0 and assume it is essentially homogeneous. Let $\operatorname{Sol}_{\text {ext }}\left(R^{k}\right)$ be defined as in Theorem 7.2.2. The map

$$
\operatorname{Sol}_{\text {ext }}\left(R^{k}\right) \ni \dot{u} \mapsto p_{r}\left(\left(\left.R^{k}\right|_{r}\right)^{*} R^{k}\right) \dot{u} \in \operatorname{Sol}_{\text {ext }}\left(R^{k}\right)
$$

is linear, where $p_{r}$ is as in Proposition 7.2.4 and $k$ is taken to be sufficiently high.

Proof. Let $r=\operatorname{deg} \dot{u}$ and let $k \in \mathbb{N}_{0}$ be such that $R^{k} u=0$. Analogously to the proof of Proposition 7.2.4, one shows that for any $\dot{u} \in \operatorname{Sol}_{\text {ext }}\left(R^{k}\right), r^{\prime} \geq r$ and $k^{\prime} \geq k$,

$$
\left[p_{r^{\prime}}\left(\left(\left.R^{k^{\prime}}\right|_{r^{\prime}}\right)^{*} R^{k^{\prime}}\right)-p_{r}\left(\left(\left.R^{k}\right|_{r}\right)^{*} R^{k}\right)\right] \dot{u} \in \operatorname{Ran}\left(R^{k}\right)^{*}
$$

and that this expression also belongs to $\operatorname{Ker}\left(\left.R\right|_{r}\right)=\operatorname{Ker}\left(\left.R\right|_{r}\right)^{k}=\left(\operatorname{Ran}\left(\left.R^{k}\right|_{r}\right)^{*}\right)^{\perp}$, hence vanishes.

In the case when on-shell extensions for $R$ exist, they can also be obtained as follows using the resolvent of $\left.R\right|_{r}$.

Proposition 7.2.6. Let $R$ be an operator of essential order 0. Assume $u \in \mathscr{D}^{\prime}\left(\dot{\mathbb{R}}^{n}\right)$ has degree of divergence $r<\infty$, satisfies $R u=0$ and has on-shell extensions. If $\left.R\right|_{r}$ is normal then

$$
\ddot{u}:=\lim _{\varepsilon \rightarrow 0}\left(1-\left[\left.(R-\mathrm{i} \varepsilon)\right|_{r}\right]^{-1} R\right) \dot{u}
$$

is an on-shell extension, where $\dot{u}$ is an arbitrary extension of $u$ to $\mathbb{R}^{n}$ with the same degree of divergence.

Proof. By continuity of $R$,

$$
\begin{aligned}
R \ddot{u} & =R \lim _{\varepsilon \rightarrow 0}\left(1-\left[\left.(R-\mathrm{i} \varepsilon)\right|_{r}\right]^{-1} R\right) \dot{u}=\lim _{\varepsilon \rightarrow 0}\left(1-R\left[\left.(R-\mathrm{i} \varepsilon)\right|_{r}\right]^{-1}\right) R \dot{u} \\
& =\lim _{\varepsilon \rightarrow 0}\left(1-\left(\left.R\right|_{r}\right)\left[\left.(R-\mathrm{i} \varepsilon)\right|_{r}\right]^{-1}\right) R \dot{u}=\lim _{\varepsilon \rightarrow 0}(-\mathrm{i} \varepsilon)\left[\left.(R-\mathrm{i} \varepsilon)\right|_{r}\right]^{-1} R \dot{u} .
\end{aligned}
$$

The operator $(-\mathrm{i} \varepsilon)\left[\left.(R-\mathrm{i} \varepsilon)\right|_{r}\right]^{-1}$ converges to the orthogonal projection to $\left.\operatorname{Ker} R\right|_{r}=$ $\operatorname{Ker}\left(\left.R\right|_{r}\right)^{*}$ and by assumption $\left.R \dot{u} \in \operatorname{Ran} R\right|_{r}$, therefore the above expression vanishes.

Let us now examine the special case when $\left.R\right|_{r}$ is self-adjoint. Then, the operator $p_{r}\left(\left(\left.R\right|_{r}\right)^{*} R\right)$ in Theorem 7.2 .2 can be replaced by $p_{r}\left(R^{2}\right)$. The gain is that $p_{r}\left(R^{2}\right)$ makes sense as an operator acting on arbitrary distributions. Even better, it is well defined as an operator from $\mathscr{D}_{\mathcal{K}}^{\prime}\left(\mathbb{R}^{n}\right)$ to $\mathscr{D}^{\prime}\left(\mathbb{R}^{n}\right)$, where $\mathcal{K}=\operatorname{Ker}\left(\left.R\right|_{r}\right)$. This fact can be used to construct directly an on-shell extension $\ddot{u}$, that is without referring to some generic extension $\dot{u}$. In the following proposition, the polynomial $p_{r}$ (defined using the eigenvalues of $R^{2}$ ) is replaced by a polynomial $b_{r}$ defined using the eigenvalues of $R$, which makes the formulae slightly more compact.

Proposition 7.2.7. Let $R$ be of essential order 0 and assume $\left(\left.R\right|_{r}\right)^{*}=\left.R\right|_{r}$. Assume $u \in \mathscr{D}^{\prime}\left(\dot{\mathbb{R}}^{n}\right)$ has degree of divergence $r<\infty$ and satisfies $R u=0$ on $\dot{\mathbb{R}}^{n}$. Suppose that $u$ has an on-shell extension for $R$. Denote $\mathcal{K}=\operatorname{Ker}\left(\left.R\right|_{r}\right)$ and let $W^{\mathrm{t}}: \mathscr{D}\left(\mathbb{R}^{n}\right) \rightarrow$ $\mathscr{D}_{\mathcal{K}}\left(\mathbb{R}^{n}\right)$ be a projection. Set

$$
\ddot{u}:=W b_{r}(R) \tilde{u}
$$


where $\tilde{u}$ is the unique extension of $u$ in $\mathscr{D}_{r}^{\prime}\left(\mathbb{R}^{n}\right)$ with $\operatorname{deg} \tilde{u}=\operatorname{deg} u$, and $b_{r}$ is the polynomial

$$
b_{r}(z)=\prod_{\left.\lambda \in \operatorname{sp} R\right|_{r} \backslash\{0\}}(1-z / \lambda) .
$$

Then $\ddot{u}$ is an extension of $u$ in $\mathscr{D}^{\prime}\left(\mathbb{R}^{n}\right)$ with $\operatorname{deg} \ddot{u}=\operatorname{deg} u$ and $R \ddot{u}=0$ on $\mathbb{R}^{n}$.

Proof. The projection $W^{\mathrm{t}}$ can be written as

$$
W^{\mathrm{t}} \varphi=\varphi-\sum_{i \in \mathcal{I}}\left\langle w_{i}, \varphi\right\rangle \phi_{i}
$$

where $\left\{w_{i}\right\}_{i \in \mathcal{I}}$ is a basis of $\mathcal{K}=\operatorname{Ker}\left(\left.R\right|_{r}\right)=\operatorname{Ran}\left(b_{r}\left(\left.R\right|_{r}\right)\right)$ and $\left\{\phi_{i}\right\}_{i \in \mathcal{I}}$ are elements of $\mathscr{D}\left(\mathbb{R}^{n}\right)$ such that $\left\langle w_{i}, \phi_{j}\right\rangle=\delta_{i j}$. Let us choose $\left\{v_{i}\right\}_{i \in \mathcal{I}}, v_{i} \in \mathcal{K}$ in such way that $b_{r}(R) v_{i}=w_{i}$ (which is always possible for dimensional reasons). Set

$$
V^{\mathrm{t}} \varphi:=\varphi-\sum_{i \in \mathcal{I}}\left\langle v_{i}, \varphi\right\rangle b_{r}\left(R^{\mathrm{t}}\right) \phi_{i}
$$

Since $\left\langle v_{i}, b_{r}\left(R^{\mathrm{t}}\right) \phi_{j}\right\rangle=\left\langle b_{r}(R) v_{i}, \phi_{j}\right\rangle=\delta_{i j}, V^{\mathrm{t}}: \mathscr{D}\left(\mathbb{R}^{n}\right) \rightarrow \mathscr{D}_{\mathcal{K}^{\perp}}\left(\mathbb{R}^{n}\right)$ is a projection and a short computation gives $V^{\mathrm{t}} b_{r}\left(R^{\mathrm{t}}\right)=b_{r}\left(R^{\mathrm{t}}\right) W^{\mathrm{t}}$. Since $b_{r}\left(R^{\mathrm{t}}\right)$ maps $\mathscr{D}\left(\mathbb{R}^{n}\right)$ to $\mathscr{D}_{\mathcal{K}^{\perp}}\left(\mathbb{R}^{n}\right)$, we have $Z^{\mathrm{t}} b_{r}\left(R^{\mathrm{t}}\right)=b_{r}\left(R^{\mathrm{t}}\right)$ for any projection $Z^{\mathrm{t}}: \mathscr{D}\left(\mathbb{R}^{n}\right) \rightarrow \mathscr{D}_{\mathcal{K}^{\perp}}\left(\mathbb{R}^{n}\right)$. Thus,

$$
\ddot{u}=W b_{r}(R) \tilde{u}=b_{r}(R) Z V \tilde{u} .
$$

By 3. of Lemma 7.1.2, $V^{\mathrm{t}}$ maps $\mathscr{D}_{\mathcal{K}^{\perp}}\left(\mathbb{R}^{n}\right)$ to $\mathscr{D}_{r}\left(\mathbb{R}^{n}\right)$, hence $V^{\mathrm{t}} Z^{\mathrm{t}}$ maps $\mathscr{D}\left(\mathbb{R}^{n}\right)$ to $\mathscr{D}_{r}\left(\mathbb{R}^{n}\right)$. It follows that $\dot{u}:=Z V \tilde{u}$ is an element of $\mathscr{D}^{\prime}\left(\mathbb{R}^{n}\right)$ and $\ddot{u}=b_{r}(R) \dot{u}$. By Theorem 7.2.2 (with $b_{r}(R)$ playing the role of $p_{r}\left(R^{2}\right)$ ), $\ddot{u}$ is an element of $\mathscr{D}^{\prime}\left(\mathbb{R}^{n}\right)$ with the required properties.

In the next theorem we give several conditions on $R$ which ensure the existence of on-shell extensions for all degrees of divergence $r \geq 0$. Note that these conditions are of rather different nature.

TheOREM 7.2.8. Let $R$ be an operator of essential order 0 , let $u \in \mathscr{D}^{\prime}\left(\dot{\mathbb{R}}^{n}\right)$ have degree of divergence $r<\infty$ and assume $R u=0$ on $\dot{\mathbb{R}}^{n}$. Assume at least one of the following holds:

1) $\operatorname{Ker}\left(\left.R\right|_{r}\right)=\{0\}$;

2) $R^{\mathrm{t}}$ maps polynomials of degree $\leq r$ to polynomials of degree $\leq r$ and $\operatorname{supp} u$ is compact;

3) $R^{\mathrm{t}}$ maps polynomials of degree $\leq r$ to polynomials of degree $\leq r$ and there exist $\psi, \phi \in \mathscr{D}\left(\mathbb{R}^{n}\right)$ s.t. $\psi \equiv 1$ in a neighbourhood of 0 and $R^{\mathrm{t}} \psi=\phi R^{\mathrm{t}}$;

Then $u$ admits an on-shell extension, i.e. an extension $\ddot{u} \in \mathscr{D}^{\prime}\left(\mathbb{R}^{n}\right)$ s.t. $\operatorname{deg} \ddot{u}=r$ and $R \ddot{u}=0$ on $\mathbb{R}^{n}$.

Proof. 1) If $\operatorname{Ker}\left(\left.R\right|_{r}\right)=\{0\}$ then $\operatorname{Ran}\left(\left.R\right|_{r}\right)=\mathscr{D}^{\prime}(\{0\})_{\leq r}$ and b) of Theorem 7.2.2 is trivially satisfied.

2) Let $\dot{u} \in \mathscr{D}^{\prime}\left(\mathbb{R}^{n}\right)$ be an arbitrary extension of $u$ with the same degree of divergence. We want to show that b) of Theorem 7.2.2 is satisfied, or equivalently

$$
(R \dot{u} \mid v)_{r}=0 \quad \forall v \in\left(\operatorname{Ran}\left(\left.R\right|_{r}\right)\right)^{\perp} .
$$


Using that $u$ is compactly supported, we obtain

$$
(R \dot{u} \mid v)_{r}=\left\langle\overline{R \dot{u}}, \mathcal{S}_{r} v\right\rangle=\left\langle\overline{\dot{u}}, \overline{R^{\mathrm{t}}} \mathcal{S}_{r} v\right\rangle
$$

To show that the expression above vanishes, let us remark that $\left.v \in \operatorname{Ran}\left(\left.R\right|_{r}\right)\right)^{\perp}$ means

$$
0=(R w \mid v)_{r}=\left\langle\overline{R w}, \mathcal{S}_{r} v\right\rangle=\left\langle\bar{w}, \overline{R^{\mathrm{t}}} \mathcal{S}_{r} v\right\rangle \quad \forall w \in \mathscr{D}^{\prime}(\{0\})_{\leq r}
$$

which implies $\overline{R^{\mathrm{t}}} \mathcal{S}_{r} v=0$.

3) Let us show that (7.2.4) holds. We have

$$
(R \dot{u} \mid v)_{r}=\left\langle\overline{R \dot{u}}, \mathcal{S}_{r} v\right\rangle=\left\langle\overline{R \dot{u}}, \bar{\psi} \mathcal{S}_{r} v\right\rangle=\left\langle\overline{\dot{u}}, \bar{R}^{\mathrm{t}} \bar{\psi} \mathcal{S}_{r} v\right\rangle=\left\langle\overline{\dot{u}}, \overline{\phi R}^{\mathrm{t}} \mathcal{S}_{r} v\right\rangle,
$$

where in the second equality we used that $R \dot{u}$ is supported at $\{0\}$. The expression above vanishes because as previously, $\bar{R}^{\mathrm{t}} \mathcal{S}_{r} v=0$.

Let us emphasize that if condition 1) holds then the on-shell extension $\dot{u}$ is unique.

Condition 3) is satisfied if $R$ is for instance one of the infinitesimal generators of rotations.

\subsubsection{Example - homogeneous and almost homogeneous distribu-} tions. The canonical example for extension of singular distributions are homogeneous distributions. We will now show that the known results on extensions of homogeneous or almost homogeneous distributions which appear in renormalisation are easily recovered in our approach .

Proposition 7.2.9. ([Hör83], Thm 3.2.3) Let $u \in \mathscr{D}^{\prime}\left(\dot{\mathbb{R}}^{n}\right)$ be homogeneous of degree $a \in \mathbb{C}$, i.e.

$$
\left(\sum_{i=1}^{n} x_{i} \partial_{i}-a\right) u=0 \quad \text { on } \dot{\mathbb{R}}^{n}
$$

If $-\operatorname{Re} a \notin \mathbb{N}_{0}+n$ then $u$ has a unique homogeneous extension $\dot{u} \in \mathscr{D}^{\prime}\left(\mathbb{R}^{n}\right)$ (i.e. $\left(\sum_{i=1}^{n} x_{i} \partial_{i}-a\right) \dot{u}=0$ on $\left.\mathbb{R}^{n}\right)$.

Proof. Clearly, $R:=\sum_{i=1}^{n} x_{i} \partial_{i}-a$ is an operator of essential order 0. By Theorem 7.2.8, for an on-shell extension to exist it is sufficient that $\operatorname{Ker}\left(\left.R\right|_{r}\right)=\{0\}$ for all $r \in \mathbb{N}_{0}$ (and in such case it is unique). Since $\left.R\right|_{r} \delta^{(\alpha)}=R \delta^{(\alpha)}=-(|\alpha|+n+a) \delta^{(\alpha)}$, we have

$$
|\operatorname{det} R|_{r}|=| \prod_{|\alpha| \leq-\operatorname{Re} a-n}(|\alpha|+n+a) \mid,
$$

so that $-\operatorname{Re} a \notin \mathbb{N}_{0}+n$ entails $|\operatorname{det} R|_{r} \mid \neq 0$.

The following proposition concerns distributions of generalized homogeneity. It is a variant of [HW02, Lem. 4.1], see also [DF04, Prop. 4]) and [Kel10, Cor. I.15].

Proposition 7.2.10. Let $a_{1}, \ldots, a_{k} \in \mathbb{C}$. Let $u \in \mathscr{D}^{\prime}\left(\dot{\mathbb{R}}^{n}\right)$ and assume $\prod_{j=1}^{k} R\left(a_{j}\right) u=$ 0 on $\dot{\mathbb{R}}^{n}$, where $R(a):=\left(\sum_{i=1}^{n} x_{i} \partial_{i}-a\right)$. If $-\operatorname{Re} a_{j} \notin \mathbb{N}_{0}+n$, then $u$ has a unique extension $\ddot{u} \in \mathscr{D}^{\prime}\left(\mathbb{R}^{n}\right)$ s.t. $\prod_{j=1}^{k} R\left(a_{j}\right) \ddot{u}=0$ on $\mathbb{R}^{n}$.

Proof. We use Theorem 7.2.8 as in Proposition 7.2.9. We need to prove that $\prod_{j=1}^{k} R\left(a_{j}\right)$ restricted to $\mathscr{D}^{\prime}(\{0\})_{\leq r}$ has trivial kernel for $r \in \mathbb{N}_{0}$. But this readily follows from the same property for the operators $R\left(a_{j}\right)$ that was shown in the proof of Proposition 7.2.9. 
If $u$ is as above with all $a_{j}$ 's equal, one speaks of an almost homogeneous distribution (or associate homogeneous distribution, cf. [NST11] and references therein). If the $a_{j}$ 's are pairwise distinct, one speaks of a heterogeneous distribution.

Since for arbitrary $r \in \mathbb{N}_{0},\left.R(a)\right|_{r}$ is diagonal in the basis $\left\{\delta^{(\alpha)}\right\}_{|\alpha| \leq r}$, it is normal. As a straightforward corollary from Proposition 7.2.4 one recovers the following result on almost homogeneous distributions.

Proposition 7.2.11. Let $u \in \mathscr{D}^{\prime}\left(\dot{\mathbb{R}}^{n}\right)$ and assume $R(a)^{k} u=0$ on $\dot{\mathbb{R}}^{n}$. Then there exists an extension $\dot{u} \in \mathscr{D}^{\prime}\left(\mathbb{R}^{n}\right)$ with the same degree of divergence s.t. $R(a)^{k+1} \dot{u}=0$ on $\mathbb{R}^{n}$.

\subsection{On-shell extension - multiple operators}

Let us now move on to the more general problem where instead of a single operator $Q$, several operators $\left\{Q^{i}\right\}_{i=1}^{k}$ are considered.

If the operators $\left\{Q^{i}\right\}_{i=1}^{k}$ commute pairwise, one can easily generalize the results of sections 7.2 and 7.2.1. For instance, the generalization of the key part of Theorem 7.2 .2 reads:

TheOREm 7.3.1. Let $\left\{Q^{i}\right\}_{i=1}^{k}$ be a family of mutually commuting operators of arbitrary essential order. Let $u \in \mathscr{D}^{\prime}\left(\dot{\mathbb{R}}^{n}\right)$ have $r:=\operatorname{deg} u<\infty$ and let it satisfy

$$
Q^{i} u=0 \quad \text { on } \dot{\mathbb{R}}^{n}, \quad i=1, \ldots, k .
$$

The following are equivalent:

a) There is an extension $\ddot{u} \in \mathscr{D}^{\prime}\left(\mathbb{R}^{n}\right)$ of $u$ with $\operatorname{deg} \ddot{u}=\operatorname{deg} u$ such that $Q^{i} \ddot{u}=0$ on $\mathbb{R}^{n}(i=1, \ldots, k)$;

b) For all extensions $\dot{u} \in \mathscr{D}^{\prime}\left(\mathbb{R}^{n}\right)$ of $u$ with $\operatorname{deg} \dot{u}=\operatorname{deg} u$, one has

$$
Q^{j} \prod_{i=1}^{k} p_{r}^{i}\left(\left(\left.Q^{i}\right|_{r}\right)^{*} Q^{i}\right) \dot{u}=0, \quad j=1, \ldots, k,
$$

where $p_{r}^{i}$ is the polynomial $p_{r}^{i}(z):=\prod_{\lambda}(1-z / \lambda)$, the product being taken over all nonzero $\lambda \in \operatorname{sp}\left(\left(\left.Q^{i}\right|_{r}\right)\left(\left.Q^{i}\right|_{r}\right)^{*}\right)$.

In the case when the operators $Q^{i}$ do not commute pairwise, one strategy is to find a polynomial of the $Q^{i}$ s (or several mutually commuting ones), which commutes (respectively, commute) with all the $Q^{i}$ 's. If the set of solutions of this operator (respectively, the joint set of solutions of these operators) coincides with the joint set of solutions of the $Q^{i}$ 's, then one is reduced to the case of a single operator (or several mutually commuting ones). This requirement can be formulated as follows:

Assumption C. Assume there exist mutually commuting operators $\left\{C^{j}\right\}_{j=1}^{k^{\prime}}$ which are polynomials of the $Q^{i}$ 's, commute with all the $Q^{i}$ 's and satisfy

$$
\bigcap_{j=1}^{k^{\prime}} \operatorname{Ker}\left(\left.C^{j}\right|_{r}\right)=\bigcap_{i=1}^{k} \operatorname{Ker}\left(\left.Q^{i}\right|_{r}\right) .
$$

Provided mutually commuting operators exist, one inclusion is always guaranteed. The non-trivial part in the assumption is that the joint kernel of the operators $C^{j}$ 
should not be larger than that of the original operators. Observe that often in the applications, those $Q^{j}$ which do not commute among themselves form a Lie algebra, and the $C^{j}$ are the Lie algebra's Casimir operators. Below we give a criterion for existence of on-shell extensions which is particularly useful in this context.

Theorem 7.3.2. Let $\left\{R^{i}\right\}_{i=1}^{k}$ be a set of operators of essential degree 0 , let $u \in \mathscr{D}\left(\dot{\mathbb{R}}^{n}\right)$ have degree of divergence $r<\infty$ and assume

$$
R^{i} u=0 \text { on } \dot{\mathbb{R}}^{n}, \quad i=1, \ldots, k .
$$

Let $C$ be a polynomial in the variables $R^{i}(i=1, \ldots, k)$ with no term of degree one or zero. Assume that

$$
\left(\left.C\right|_{r}\right)^{*}=\left.C\right|_{r}, \quad C R^{i}=R^{i} C, \quad i=1, \ldots, k .
$$

and that the following stronger form of Assumption $C$ is satisfied

$$
\operatorname{Ker} C=\bigcap_{i=1}^{k} \operatorname{Ker} R^{i} \text {. }
$$

Then $u$ has an on-shell extension, i.e., an extension $\ddot{u} \in \mathscr{D}^{\prime}\left(\mathbb{R}^{n}\right)$ with $\operatorname{deg} u=r$ s.t. $R^{i} \ddot{u}=0$ on $\mathbb{R}^{n}$ for $i=1, \ldots, k$.

Proof. Since $\left.C\right|_{r}$ is self-adjoint, there is a polynomial $b_{r}$ s.t. $b_{r}\left(\left.C\right|_{r}\right)$ is the orthogonal projection to $\operatorname{Ker}\left(\left.C\right|_{r}\right)^{*}=\left.\operatorname{Ker} C\right|_{r}$, namely

$$
b_{r}(z)=\prod_{\left.\lambda \in \operatorname{sp} C\right|_{r} \backslash\{0\}}(1-z / \lambda) .
$$

Let $\dot{u} \in \mathscr{D}^{\prime}\left(\mathbb{R}^{n}\right)$ be an arbitrary extension of $u$ with degree of divergence $r$ and set $\ddot{u}:=b_{r}(C) \dot{u}$. Clearly, $\ddot{u}$ is an extension of $u$ with degree of divergence $r$. Moreover,

$$
R^{i} \ddot{u}=R^{i} b_{r}(C) \dot{u}=b_{r}\left(\left.C\right|_{r}\right) R^{i} \dot{u} \in \operatorname{Ker} C=\bigcap_{j=1}^{k} \operatorname{Ker} R^{j}, \quad i=1, \ldots, k
$$

hence $R^{i} R^{j} \ddot{u}=0$ for all $i, j$ and consequently $C \ddot{u}=0$, which entails $R^{i} \ddot{u}=0$.

The above theorem can be used to treat Lorentz symmetry, by taking $C=\left(x_{\mu} \partial_{\nu}-\right.$ $\left.x_{\nu} \partial_{\mu}\right)\left(x^{\mu} \partial^{\nu}-x^{\nu} \partial^{\mu}\right)$ (the quadratic Casimir for the Lorentz group) and $R_{i}$ proportional to generators of rotations and boosts. It was proved [DF04] that the kernel of this operator corresponds indeed to Lorentz invariant distributions, the operator $b_{r}(C)$ was also used therein in the construction of on-shell extensions.

7.3.1. Application - renormalisation conditions in scalar theory. As an application of our framework, we show how can one treat the symmetries which arise in a scalar quantum field theory. In this particular case our method essentially reduces to the arguments used in [DF04], except that we obtain an additional result on linearity of the map $\mathcal{R}$ defined below.

Corollary 7.3.3. Let $\operatorname{Sol}_{\text {ext }}(\mathrm{QFT})$ denote the space of all distributions $v \in \mathscr{D}^{\prime}\left(\mathbb{R}^{n}\right)$ with finite degree of divergence such that the restriction $u:=\left.v\right|_{\mathbb{R}^{n}}$ satisfies:

a) $\operatorname{deg} u=\operatorname{deg} v$,

b) $u$ is Lorentz-invariant;

c) $u$ is the finite sum of almost homogeneous distributions of integer degree. 
Then there exists a linear map $\mathcal{R}: \operatorname{Sol}_{\text {ext }}(\mathrm{QFT}) \mapsto \operatorname{Sol}_{\text {ext }}(\mathrm{QFT})$ such that for all $v \in \operatorname{Sol}_{\text {ext }}(\mathrm{QFT})$,

(1) $\mathcal{R} v=v$ on $\dot{\mathbb{R}}^{n}$

(2) $\mathcal{R} v$ is Lorentz-invariant;

(3) $\mathcal{R} v$ is the finite sum of almost homogeneous distributions. More precisely, if $v$ is almost homogeneous of degree $a \in \mathbb{Z}$ and order $k \in \mathbb{N}_{0}$ on $\dot{\mathbb{R}}^{n}$, then $\mathcal{R} v$ is almost homogeneous on $\mathbb{R}^{n}$ of degree $a$ and order $k$ if $k \notin \mathbb{N}_{0}+n$ and of order $k+1$ otherwise.

Proof. Let $C=\left(x_{\mu} \partial_{\nu}-x_{\nu} \partial_{\mu}\right)$ and $R(a)=\sum_{i=1}^{n} x_{i} \partial_{i}-a$ as in Subsection 7.2.2. For any $v \in \operatorname{Sol}_{\text {ext }}(\mathrm{QFT}), C v=0$ on $\dot{\mathbb{R}}^{n}$ and there is a sequence of non-negative integers $\left\{N_{j}\right\}_{j \in \mathbb{Z}}$ s.t. $N_{j}=0$ for almost all $j$ and $\prod_{j \in \mathbb{Z}} R(j)^{N_{j}} v=0$ on $\dot{\mathbb{R}}^{n}$. Set

$$
\mathcal{R} v=p_{r}\left(\prod_{j \in \mathbb{Z}} R(j)^{2 N_{j}}\right) b_{r}(C) v,
$$

where $r=\operatorname{deg} v, b_{r}(z):=\prod_{\lambda}(1-z / \lambda)$ where the product runs over all $\lambda \in \operatorname{sp}\left(\left.C\right|_{r}\right) \backslash$ $\{0\}, p_{r}(z):=\prod_{\lambda}(1-z / \lambda)$ where the product runs over all $\lambda \in \operatorname{sp}\left(\left.\prod_{j \in \mathbb{Z}} R(j)^{2 N_{j}}\right|_{r}\right) \backslash$ $\{0\}$. Since $\left.C\right|_{r}$ and $\left.\prod_{j \in \mathbb{Z}} R(j)^{N_{j}}\right|_{r}$ are self-adjoint for any $r$, the arguments from Proposition 7.2.5 give linearity of $\mathcal{R}$. Property 3 is proved as in subsection 7.2.2. Based on the fact that $C$ satisfies the conditions in Theorem 7.3.2, we deduce that property 2 holds. That these two properties can be satisfied simultaneously is due to the fact that the $R(j)$ commute with $C$.

7.3.2. Vector-valued distributions. Let us consider distributions in $\mathscr{D}^{\prime}\left(\dot{\mathbb{R}}^{n}, \mathbb{R}^{q}\right)$, $\mathscr{D}^{\prime}\left(\mathbb{R}^{n}, \mathbb{R}^{q}\right)$. We use the notation $\langle u, \varphi\rangle=\sum_{i=1}^{q}\left\langle u_{i}, \varphi_{i}\right\rangle$ for the pairing between $\mathscr{D}^{\prime}\left(\mathbb{R}^{n}, \mathbb{R}^{q}\right)$ and $\mathscr{D}\left(\mathbb{R}^{n}, \mathbb{R}^{q}\right)$, where $u=\left(u_{1}, \ldots, u_{q}\right) \in \mathscr{D}^{\prime}\left(\mathbb{R}^{n}, \mathbb{R}^{q}\right)$ and $\varphi=\left(\varphi_{1}, \ldots, \varphi_{q}\right) \in \mathscr{D}^{\prime}\left(\mathbb{R}^{n}, \mathbb{R}^{q}\right)$. With this notation, the definition of the scaling degree extends verbatim to the case of vector-valued distributions, and so do the results on extension of distributions. One easily sees that

$$
\operatorname{sd} u=\max _{i=1, \ldots, q}\left(\operatorname{sd} u_{i}\right) \text {. }
$$

In practice, one is often interested in the following situation. Let $G$ be a Lie group acting on $\mathbb{R}^{n}$ and consider the group action on $\mathbb{R}^{n} \times \mathbb{R}^{q}$ given by

$$
g:(x, u) \mapsto(g \cdot x, \mu(g, x) u), \quad g \in G, x \in \mathbb{R}^{n}, u \in \mathbb{R}^{q},
$$

where $\mu: G \times \mathbb{R}^{n} \rightarrow G L(q)$ satisfies

$$
\mu(g \cdot h, x)=\mu(g, h \cdot x) \mu(h, x), \quad \mu(e, x)=\mathbb{1}
$$

for all $g, h \in G, x \in \mathbb{R}^{n}$. This property of $\mu$ guarantees that (7.3.1) defines an action of $G$. In applications in renormalisation one is mostly interested in the case $\mu(g, x)$ does not depend on $x$. The associated infinitesimal generators are of the form

$$
R=\sum_{i=1}^{n} \xi^{i}(x) \frac{\partial}{\partial x^{i}}+\sum_{\alpha, \beta=1}^{q} h_{\beta}^{\alpha}(x) u^{\beta} \frac{\partial}{\partial u^{\alpha}},
$$

where $\sum_{i=1}^{n} \xi^{i}(x) \frac{\partial}{\partial x^{i}}$ is the infinitesimal generator of $G$ acting on $\mathbb{R}^{n}$, associated to 
some element $\mathbf{v}$ of the Lie algebra of $G$ and $h(x)=\left.\frac{d}{d t} \mu(\exp (\mathbf{v} t), x)\right|_{t=0}$ (see [Olv95] for details). Now, the results of sections 7.2 and 7.2.1 directly carry over, and extensions which are on-shell w.r.t. the above operator $R$ can be constructed in the same manner as described there. 



\section{CHAPTER 8}

\section{On-shell and off-shell time-ordered products}

In this final chapter, we will rephrase the link between on-shell and off-shell timeordered products within the framework of [BW12]. We consider here only the simpliest case of a scalar field on Minkowski space-time, in the absence of external potentials. To introduce the reader to the problem, let us recall the well-known fact that the time-ordered product in scalar quantum field theory (of second order) is formally written as

$$
T(\phi(x) \phi(y))=\theta\left(x^{0}-y^{0}\right) \phi(x) \phi(y)+\theta\left(y^{0}-x^{0}\right) \phi(y) \phi(x),
$$

where $\phi(x)$ is the free field and $\theta$ is the Heaviside theta distribution. By Wick's theorem, $T(\phi(x) \phi(y))$ is equal to the normal product : $\phi(x) \phi(y)$ : plus the singular distribution

$$
\theta\left(z^{0}\right) \Lambda^{(+)}(z)+\theta\left(-z^{0}\right) \Lambda^{(+)}(-z)
$$

where $z=x-y$ and $\Lambda^{(+)}$is the distributional two-point function of the Minkowski vacuum, and where the product of $\theta$ and $\Lambda^{(+)}$is well-defined as a distribution in $\mathscr{D}^{\prime}\left(\mathbb{R}^{n}\right)$ by the criterion on wave front sets (cf. Section 3.2). This is a fundamental solution of $P=\square+m^{2}$, traditionally called the Feynman propagator and denoted $E_{\mathrm{F}} \in \mathscr{D}^{\prime}\left(\mathbb{R}^{n}\right)$. One can also view $(8.0 .1)$ as a distribution on $\dot{\mathbb{R}}^{n}$. Since its degree of divergence is -2 , it admits a unique extension to $\mathbb{R}^{n}$ with the same degree of divergence as given by Theorem 7.2.2. Of course, this construction again yields the Feynman propagator on $\mathbb{R}^{n}$.

Ambiguities arise when one considers higher derivatives of the fields. For instance, applying Wick's theorem to $T\left(\partial_{x}^{\mu} \phi(x) \partial_{y}^{\nu} \phi(y)\right)$, the contribution which cannot be extended unambiguously, is

$$
-\theta\left(z^{0}\right) \partial^{\mu} \partial^{\nu} \Lambda^{(+)}(z)-\theta\left(-z^{0}\right) \partial^{\mu} \partial^{\nu} \Lambda^{(+)}(-z) .
$$

The degree of divergence of this distribution is 0 . Consequently, its extensions to $\mathbb{R}^{n}$ are no longer uniquely fixed by requiring that they have the same degree of divergence. Requiring additionally Lorentz covariance, one obtains that the most general form of any such extension is

$$
\partial^{\mu} \partial^{\nu} E_{\mathrm{F}}(z)+C g^{\mu \nu} \delta(z)
$$

where $C$ is an arbitrary constant. The choice $C=0$, or more generally, a prescription for the time-ordered product which would make it 'commute' with derivatives, seems to be the simplest one. Such a choice, however, is inconsistent with the requirement that the fields are on-shell in the sense of the equation of motion, i.e. in our language on-shell w.r.t. the Klein-Gordon operator, $\left(\square+m^{2}\right) \phi=0$. Indeed, setting $C=0$ 
would imply for instance

$$
\left(\square_{x}+m^{2}\right) T(\phi(x) \phi(y))=\left(\square_{x}+m^{2}\right) E_{\mathrm{F}}(x-y)=-\mathrm{i} \delta(x-y),
$$

whereas the Klein-Gordon on-shell condition yields $T\left(\left(\square_{x}+m^{2}\right) \phi(x) \phi(y)\right)=0$.

Most of the physics literature uses on-shell time-ordered products. The off-shell formalism, developed in [DF03, DF04], is based on a time-ordered product which commutes with the derivatives of the fields. For this reason it has many advantages over the on-shell formalism, an especially remarkable one being the possiblity of writing in a compact form the so-called Master Ward Identity, which serves as a universal renormalisation condition [DF03]. Another feature of off-shell time-ordered products is consistency with the requirement that the $S$-matrix should depend only on the action, i.e., on the integral $\int_{M} \eta(x) \mathscr{L}(x) d \Omega_{g}$, rather than on the Lagrangian density $\mathscr{L}(x)$.

\subsection{Map relating off-shell and on-shell time-ordered products}

Recall from Chapter 6 that the on-shell time-ordered product $T_{\text {on }}$ of order $k$ is a map from $\mathcal{P}_{0}^{\otimes k}$ to operator-valued distributions defined by a set of axioms. Off-shell time-ordered products are by definition maps from $\mathcal{P}^{\otimes k}$ that satisfy an analogous set of axioms and that commute with derivatives, see [DF03] for a detailed discussion ${ }^{1}$. In the present setting, we are merely interested in the following result which relates $T_{\text {on }}$ and $T_{\text {off }}$ :

Theorem 8.1.1 ([DF03, DF04, BD08]). There exists a unique linear map $S \mapsto \chi(S)$ from differential operators with constant coefficients to differential operators with constant coefficients such that:

a) $\chi\left(S\left(\square+m^{2}\right)\right)=0$ for all $S$;

b) for any $S=\partial_{\mu_{1}} \ldots \partial_{\mu_{k}}, \chi(S)$ transforms under Lorentz transformations as $S$;

c) ord $\chi(S) \leq$ ord $S$ for all $S$;

d) there is a linear map $S \mapsto \chi_{1}(S)$ s.t. $\chi(S)-S=\chi_{1}(S)\left(\square+m^{2}\right)$.

Now, on-shell and off-shell time-ordered products of order $k$ are related by

$$
T_{\text {on }}\left(A_{1} \otimes \cdots \otimes A_{k}\right)=T_{\text {off }}\left(\sigma\left(A_{1}\right) \otimes \cdots \otimes \sigma\left(A_{k}\right)\right), \quad A_{i} \in \mathcal{P}_{0},
$$

where $\sigma: \mathcal{P}_{0} \rightarrow \mathcal{P}$ is the unique linear algebra homomorphism s.t. $\sigma \pi(S \varphi)=\chi(S) \varphi$ for all $S, \varphi$.

This recurrence has been solved in [BD08] in an explicit way. Although the result is certainly well-suited for practical use, it involves long combinatorial expressions which do not seem to have a deeper interpretation. For the sake of completeness, we quote below the explicit formula for $\chi$ obtained in [BD08].

\footnotetext{
${ }^{1}$ The requirement that $T_{\text {off }}$ should commute with derivatives is called Action Ward Identity in [DF03], the concept and the name is attributed therein to Stora.
} 
TheOREM 8.1.2 ([BD08]). The map $\chi$ from Theorem 8.1.1 is given by

$$
\chi\left(\partial_{\mu_{1}} \ldots \partial_{\mu_{k}}\right)=\sum_{j=0}^{k / 2} \alpha_{j}^{k} P_{j}^{k}\left(\partial_{\mu_{1}} \ldots \partial_{\mu_{k}}\right),
$$

where $P_{j}^{k}(S)=\frac{1}{j !} \Lambda^{j}(S), \quad \Lambda\left(\partial_{\mu_{1}} \ldots \partial_{\mu_{k}}\right)=\sum_{i<j} g_{\mu_{i} \mu_{j}} \partial_{\mu_{1} \ldots \hat{i} \ldots \hat{j} \ldots \mu_{k}}$ (where $\hat{i}$ means $\mu_{i}$ is removed), $a_{0}^{k}=0$ and for $j>0$

$$
\alpha_{j}^{k}=(-1)^{j}\left(\square+m^{2}\right) \sum_{p=0}^{j-1}\left(\begin{array}{c}
j-1 \\
p
\end{array}\right) m^{2 p} \square^{j-1-p} \prod_{q=0}^{j-1}(n+2 k-2 p-2 q-4)^{-1} .
$$

In particular, at the lowest orders, (8.1.1) gives

$$
\chi(\mathbf{1})=\mathbf{1}, \quad \chi\left(\partial_{\mu}\right)=\partial_{\mu}, \quad \chi\left(\partial_{\mu} \partial_{\nu}\right)=\partial_{\mu} \partial_{\nu}-n^{-1} g_{\mu \nu}\left(\square+m^{2}\right),
$$

which entails $\chi\left(\square+m^{2}\right)=0$ as required.

8.1.1. Construction of the map in the present setting. In our setting, the problem can be formulated as follows. We are given a fundamental solution $E_{\mathrm{F}} \in \mathscr{D}^{\prime}\left(\mathbb{R}^{n}\right)$ of $Q=\square+m^{2}$. Given a partial differential operator $S$ with constant coefficients, we want to replace $S E_{\mathrm{F}}$ with a distribution which agrees with the latter on $\dot{\mathbb{R}}^{n}$ and is, moreover, 0 when $S=\left(\square+m^{2}\right)$. More precisely, the question is to associate to each differential operator $S$ with constant coefficients a distribution $\Theta(S) \in \mathscr{D}^{\prime}\left(\mathbb{R}^{n}\right)$ such that:

(1) $\Theta(S)=S E_{\mathrm{F}}$ on $\dot{\mathbb{R}}^{n}$

(2) $\operatorname{deg} \Theta(S) \leq \operatorname{deg} S E_{\mathrm{F}}$;

(3) the assignment $S \mapsto \Theta(S)$ is linear;

(4) $\Theta(S)$ is Lorentz-covariant;

(5) $\Theta\left(S\left(\square+m^{2}\right)\right)=0$ for all $S$.

Provided that $\Theta(S)$ satisfies the above properties, it can be used to define directly the on-shell time-ordered product of order two. It will, however, be more convenient to relate $\Theta(S)$ to the map considered in [BD08] which gives the connection between between the on-shell and off-shell time-ordered products of order $k$, cf. Theorem 8.1.1 and 8.1.2.

Let us recall from the previous section that we can find a map $\mathcal{R}$ that assigns to a distribution $u$ with $Q u=0$ on $\dot{\mathbb{R}}^{n}$ a distribution $\mathcal{R} u$ which is 'as close to being a solution of $Q$ on $\mathbb{R}^{n}$ as possible'. The same idea is used in the construction of the map $S \mapsto \Theta(S)$ in the theorem below. The least trivial property is linearity - although we are interested in operators which are not necessarily essentially homogeneous in the sense of Definition 7.1.6, this problem can be overcome making use of the fact that we consider here only differential operators with constant coefficients.

Theorem 8.1.3. Let $Q: \mathscr{D}^{\prime}\left(\mathbb{R}^{n}\right) \rightarrow \mathscr{D}^{\prime}\left(\mathbb{R}^{n}\right)$ be a nonzero differential operator of order $q$ with constant coefficients and assume $v \in \mathscr{D}^{\prime}\left(\mathbb{R}^{n}\right)$ satisfies $\operatorname{deg} v=-q$ and $Q v=c \delta$ for some $c \in \mathbb{C}$. Assign to a differential operator $S$ of order $s$ with constant coefficients the distribution

$$
\Theta(S):=p_{s}\left(\left(\left.Q\right|_{s}\right)^{*} Q\right) S v \in \mathscr{D}^{\prime}\left(\mathbb{R}^{n}\right),
$$


where $p_{s}$ is the polynomial $p_{s}(z)=\prod_{\lambda}(1-z / \lambda)$, the product being taken over all nonzero $\lambda \in \operatorname{sp}\left(\left(\left.Q\right|_{s}\right)\left(\left.Q\right|_{s}\right)^{*}\right)$. Then $\Theta(S Q)=0$ for each differential operator $S$ with constant coefficients. Moreover, the mapping $S \mapsto \Theta(S)$ is linear.

Proof. To prove $\Theta(S Q)=0$, let us remark that this is equivalent to $Q \Theta(S Q)=0$, as $Q$ has no nonzero solutions in $\mathscr{D}^{\prime}(\{0\})$. We have

$$
Q \Theta(S Q)=Q p_{s+q}\left(\left(\left.Q\right|_{s+q}\right)^{*} Q\right) S Q v=p_{s+q}\left(Q\left(\left.Q\right|_{s+q}\right)^{*}\right) Q S \delta .
$$

Clearly, $Q S \delta \in \operatorname{Ran}\left(\left.Q\right|_{s+q}\right)=\left(\operatorname{Ker}\left(\left.Q\right|_{s+q}\right)^{*}\right)^{\perp}$, so it is projected out by $p_{s+q}\left(Q\left(\left.Q\right|_{s+q}\right)^{*}\right)$.

For linearity, we have to prove that if $s^{\prime} \geq s$ then $p_{s^{\prime}}\left(\left(\left.Q\right|_{s^{\prime}}\right)^{*} Q\right) S v$ equals $p_{s}\left(\left(\left.Q\right|_{s}\right)^{*} Q\right) S v$. Because $Q$ has no nonzero solutions in $\mathscr{D}^{\prime}(\{0\})$, this is equivalent to

$$
Q\left[p_{s^{\prime}}\left(\left(\left.Q\right|_{s^{\prime}}\right)^{*} Q\right)-p_{s}\left(\left(\left.Q\right|_{s}\right)^{*} Q\right)\right] S v=0 .
$$

One has

$$
\begin{aligned}
& Q\left[p_{s^{\prime}}\left(\left(\left.Q\right|_{s^{\prime}}\right)^{*} Q\right)-p_{s}\left(\left(\left.Q\right|_{s}\right)^{*} Q\right)\right] S v \\
= & {\left[p_{s^{\prime}}\left(Q\left(\left.Q\right|_{s^{\prime}}\right)^{*}\right)-p_{s}\left(Q\left(\left.Q\right|_{s}\right)^{*}\right)\right] S Q v } \\
= & c\left[p_{s^{\prime}}\left(Q\left(\left.Q\right|_{s^{\prime}}\right)^{*}\right)-p_{s}\left(Q\left(\left.Q\right|_{s}\right)^{*}\right)\right] S \delta .
\end{aligned}
$$

To prove that the last expression vanishes it suffices to show that $p_{s^{\prime}}\left(Q\left(\left.Q\right|_{s^{\prime}}\right)^{*}\right)$ and $p_{s}\left(Q\left(\left.Q\right|_{s}\right)^{*}\right)$ coincide on $\mathscr{D}^{\prime}(\{0\})_{\leq s}$. Indeed, $p_{s^{\prime}}\left(Q\left(\left.Q\right|_{s^{\prime}}\right)^{*}\right)$ is the orthogonal projection to $\left(\operatorname{Ran}\left(\left.Q\right|_{s^{\prime}}\right)\right)^{\perp_{s^{\prime}+q}}$, where ${ }^{\perp_{r}}$ denotes the orthogonal complement in $\mathscr{D}^{\prime}(\{0\})_{\leq r}$. Analogously, $\left.p_{s}\left(Q\left(\left.Q\right|_{s}\right)\right)^{*}\right)$ is the orthogonal projection to $\left(\operatorname{Ran}\left(\left.Q\right|_{s}\right)\right)^{\perp_{s+q}}$. Moreover,

$$
\left(\operatorname{Ran}\left(\left.Q\right|_{s^{\prime}}\right)\right)^{\perp_{s^{\prime}+q}} \cap \mathscr{D}^{\prime}(\{0\})_{\leq s}=\left(\operatorname{Ran}\left(\left.Q\right|_{s}\right)\right)^{\perp_{s+q}} \cap \mathscr{D}^{\prime}(\{0\})_{\leq s} .
$$

Indeed, suppose that $v \in \mathscr{D}^{\prime}(\{0\})_{\leq s}$ is such that $(v \mid Q w)_{s+q}=0$ for all $w \in \mathscr{D}^{\prime}(\{0\})_{\leq s}$. Then each $w^{\prime} \in \mathscr{D}^{\prime}(\{0\})_{\leq s^{\prime}}$ can be written as $w^{\prime}=w+w^{\prime \prime}$ with $w \in \mathscr{D}^{\prime}(\{0\})_{\leq s}$ and $w^{\prime \prime} \in\left(\mathscr{D}^{\prime}(\{0\})_{\leq s^{\prime}} \backslash \mathscr{D}^{\prime}(\{0\})_{\leq s}\right) \cup\{0\}$ and we have

$$
\left(v \mid Q w^{\prime}\right)_{s^{\prime}+q}=(v \mid Q w)_{s+q}+\left(v \mid Q w^{\prime \prime}\right)_{s^{\prime}+q}=0,
$$

where the first term in the sum equals zero by assumption and the second one vanishes because $Q w^{\prime \prime} \in\left(\mathscr{D}^{\prime}(\{0\})_{\leq s^{\prime}} \backslash \mathscr{D}^{\prime}(\{0\})_{\leq s}\right) \cup\{0\}$. The other inclusion in (8.1.4) is trivial.

The next lemma gives the connection between the map $S \mapsto \Theta(S)$ and a generalized version of the map $\chi$ from Theorem 8.1.1 and 8.1.2.

Lemma 8.1.4. Let $Q$ and $v$ be as in Theorem 8.1.3. There is a linear map $S \mapsto \chi(S)$ on the space of differential operators with constant coefficients, s.t. ord $\chi(S) \leq \operatorname{ord} S$ and $\Theta(S)=\chi(S) v$ for all $S$.

Proof. Since $\Theta(S)-S v \in \mathscr{D}^{\prime}(\{0\})_{\leq s-q}$, it can be written as $\chi_{1}(S) \delta$ for some differential operator $\chi_{1}(S)$ with constant coefficients of order $s-q$. More precisely, $\chi_{1}(S)=$ $\mathcal{S}_{s-q}[\Theta(S)-S v](-\partial)$, hence the assignment $S \mapsto \chi_{1}(S)$ is linear. The map $S \mapsto$ $\chi(S):=\chi_{1}(S) Q+S$ satisfies the required properties. 
Setting $Q=\square+m^{2}$ and $v=E_{\mathrm{F}}$, it follows automatically that $\chi$ satisfies the conditions given in Theorem 8.1.1 except Lorentz covariance. But it easy to see that $\chi$ is defined purely using Lorentz covariant quantities.

Corollary 8.1.5. Let $Q=\square+m^{2}, v=E_{\mathrm{F}}$, let $\Theta(S)$ be as in Theorem 8.1.3 and $\chi$ as in Lemma 8.1.4. Then $\chi$ satisfies the conditions listed in Theorem 8.1.1.

The adjoint of $\left.\left(\square+m^{2}\right)\right|_{r}$ is easily computed - one has $\left[\left.\left(\square+m^{2}\right)\right|_{r}\right]^{*}=$ $P_{r}\left[\left.\left(x_{\mu} x^{\mu}+m^{2}\right)\right|_{r+2}\right]$, where $P_{r}$ is the orthogonal projection to $\mathscr{D}^{\prime}(\{0\})_{\leq r}$. In particular if $m=0$, since $x_{\mu} x^{\mu}$ maps $\mathscr{D}^{\prime}(\{0\})_{\leq r+2}$ to $\mathscr{D}^{\prime}(\{0\})_{\leq r}$, this simplifies to $\left(\left.\square\right|_{r}\right)^{*}=\left.x_{\mu} x^{\mu}\right|_{r+2}$. Consequently the distribution $\Theta(S)$ from Theorem 8.1.3 equals

$$
\Theta(S)=p_{s}\left(x_{\mu} x^{\mu} \square\right) S E_{\mathrm{F}} .
$$

(Without the obligation to restrict $x_{\mu} x^{\mu}$ to a subspace of $\mathscr{D}^{\prime}(\{0\})$ ).

We expect that the method can be extended with no difficulty to the case of spin- $1 / 2$ and gauge fields, cf. the remarks in [BD08]. The generalization to curved space-time or external potentials remains however an open problem. 



\section{Bibliography}

[ABHN11] W. Arendt, C. Batty, M. Hieber, and F. Neubrander, Vector-valued Laplace Transforms and Cauchy Problems, Springer, 2011.

[AS71] H. Araki and M. Shiraishi, On Quasifree States of the Canonical Commutation Relations (I), Publ. Res. Inst. Math. Sci. 7 (1971), no. 1, 105-120.

[BD08] C. Brouder and M. Dütsch, Relating on-shell and off-shell formalisms in perturbative quantum field theory, J. Math. Phys. 49 (2008), no. 5, 052303.

[BF00] R. Brunetti and K. Fredenhagen, Microlocal analysis and interacting quantum field theories: renormalization on physical backgrounds, Comm. Math. Phys. 208 (2000), 623-661.

[BFK96] R. Brunetti, K. Fredenhagen, and M. Köhler, The microlocal spectrum condition and Wick polynomials of free fields on curved spacetimes, Comm. Math. Phys. 180 (1996), no. 3, 633-652.

[BG08] D. Buchholz and H. Grundling, The resolvent algebra: A new approach to canonical quantum systems, J. Funct. Anal. 254 (2008), 2725-2779.

[BG11] C. Bär and N. Ginoux, Classical and quantum fields on Lorentzian manifolds, Springer Proc. Math. 17 (2011), 359.

[BGP08] C. Bär, N. Ginoux, and F. Pfäffle, Wave Equations on Lorentzian Manifolds and Quantization, Springer, 2008.

[Bog74] J. Bognar, Indefinite Inner Product Spaces, Springer, 1974.

[Bor66] H.-J. Borchers, Comm. Math Phys 2 (1966), 49.

[BP57] N. N. Bogoliubov and O. S. Parasiuk, Über die Multiplikation der Kausalfunktionen in der Quantentheorie der Felder, Acta Math. 97 (1957), no. 1, 227-266.

[BR97] O. Bratteli and D. W. Robinson, Operator Algebras and Quantum Statistical Mechanics Vol.2: Equilibrium states. Statistical mechanics, Springer-Verlag, 1997.

[Bro83] P. Broadbridge, Existence theorems for Segal quantization via spectral theory in Krein space, Austral. Math. Soc. Ser. B 24 (1983), 439-460.

[BS59] N. N. Bogoliubov and D. V. Shirkov, Introduction to the Theory of Quantized Fields, vol. 59, Interscience New York, 1959.

[BS05] A. N. Bernal and M. Sánchez, Smoothness of time functions and the metric splitting of globally hyperbolic spacetimes, Comm. Math. Phys. 257 (2005), no. 1, $43-50$.

[BS07] — Globally hyperbolic spacetimes can be defined as 'causal' instead of 'strongly causal', Class. Quantum Grav. 24 (2007), no. 3, 745.

[BSZ92] J. Baez, I. Segal, and Z. Zhuo, Introduction to Algebraic and Constructive Quantum Field Theory, Princeton University Press, 1992.

[BW12] D. Bahns and M. Wrochna, On-shell extension of distributions, acc. in Ann. Henri Poincaré (2012). 
[Che73] P. Chernoff, Essential self-adjointness of powers of generators of hyperbolic equations, J. Funct. Anal. 12 (1973), no. 4, $401-414$.

[Dav95] E. B. Davies, The functional calculus, J. London Math. Soc 2 (1995), 166-176.

[Der06] J. Dereziński, Introduction to representations of the canonical commutation and anticommutation relations, Large Coulomb Systems, Springer, 2006, pp. 63-143.

[DF01a] M. Dütsch and K. Fredenhagen, Algebraic quantum field theory, perturbation theory, and the loop expansion, Comm. Math. Phys. 219 (2001), no. 1, 5-30.

[DF01b] _ Perturbative algebraic quantum field theory, and deformation quantization, Mathematical Physics in Mathematics and Physics: Quantum and Operator Algebraic Aspects, Fields Institute Communications, vol. 30, American Mathematical Society, 2001.

[DF03] _ The Master Ward Identity and generalized Schwinger-Dyson equation in classical field theory, Comm. Math. Phys. 243 (2003), no. 2, 275-314.

[DF04] M. Dütsch and K. Fredenhagen, Causal perturbation theory in terms of retarded products, and a proof of the Action Ward Identity, Rev. Math. Phys. 16 (2004), 1291-1348.

[DG12] M. Dütsch and J. M. Gracia-Bondía, On the assertion that PCT violation implies Lorentz non-invariance, Phys. Lett. B 711 (2012), no. 5, 428-433.

[DG13] J. Dereziński and C. Gérard, Mathematics of Quantization and Quantum Fields, Cambridge University Press, 2013.

[DH72] J. Duistermaat and L. Hörmander, Fourier integral operators. II, Acta Math. 128 (1972), no. 128, 183-269.

[Dim82] J. Dimock, Dirac quantum fields on a manifold, Trans. AMS 269 (1982), 133-147.

[DMP05] C. Dappiaggi, V. Moretti, and N. Pinamonti, Rigorous steps towards holography in asymptotically flat space-times, arXiv preprint gr-qc/0506069 (2005).

[DMP09] _ Distinguished quantum states in a class of cosmological space-times and their Hadamard property, J. Math. Phys. 50 (2009).

[DMP11] _ Rigorous construction and Hadamard property of the Unruh state in Schwarzschild space-time, Adv. Theor. Math. Phys. 15 (2011), no. 2, 355-447.

[EG73] H. Epstein and V. Glaser, The role of locality in perturbation theory, Ann. Inst. H. Poincaré A 19 (1973), 211-295.

[Est98] R. Estrada, Regularization of distributions, J. Math. \& Math. Sci. 21 (1998), no. $4,625-636$.

[FNW81] S. A. Fulling, F. J. Narcowich, and R. M. Wald, Singularity structure of the two-point function in quantum field theory in curved space-time, Ann. Phys. 136 (1981), 243-272.

[Fri75] F. G. Friedlander, The Wave Equation on a Curved Space-Time, vol. 2, Cambridge University Press, 1975.

[Ful89] S. A. Fulling, Aspects of Quantum Field Theory in Curved Spacetime, Cambridge University Press, 1989.

[FV58] H. Feshbach and F. Villars, Elementary relativistic wave mechanics of spin 0 and spin 1/2 Particles, Rev. Mod. Phys. 30 (1958), 24-45.

[Gér12] C. Gérard, Scattering theory for Klein-Gordon equations with non-positive energy, Ann. Henri Poincaré 13 (2012), no. 4, 883-941.

[GGH12] V. Georgescu, C. Gérard, and D. Häfner, Boundary values of resolvents of selfadjoint operators in Krein spaces, arXiv preprint arXiv:1211.0791 (2012). 
[GMR85] W. Greiner, B. Müller, and J. Rafelski, Quantum Electrodynamics of Strong Fields, Lecture Notes in Physics, vol. 440, Springer, 1985.

[Gra03] J. M. Gracia-Bondía, Improved Epstein-Glaser renormalization in coordinate space I. Euclidean framework, Math. Phys. Anal. Geom. 6 (2003), 59-88.

[GW13] C. Gérard and M. Wrochna, Construction of Hadamard states by pseudodifferential calculus, acc. in Comm. Math. Phys. (2013).

[Haa59] R. Haag, Discussion des "axiomes" et des proprietés asymptotiques d'une théorie des champs locale avec particules composées, Colloque international sur les problemes mathématiques de la théorie des champs, vol. 85, 1959.

[Hac10] T.-P. Hack, On the Backreaction of Scalar and Spinor Quantum Fields in Curved Spacetimes - From the Basic Foundations to Cosmological Applications, Ph.D. thesis, DESY-THESIS-2010-042, 2010.

[HK64] R. Haag and D. Kastler, An algebraic approach to quantum field theory, J. Math. Phys. 5 (1964), 848.

[HM12a] T.-P. Hack and M. Makedonski, A No-Go theorem for the consistent quantization of spin 3/2 fields on general curved spacetimes, Phys. Lett. B 718 (2012), no. 4-5, $1465-1470$.

[HM12b] T.-P. Hack and V. Moretti, On the stress-energy tensor of quantum fields in curved spacetimescomparison of different regularization schemes and symmetry of the Hadamard/Seeley-DeWitt coefficients, J. Phys. A 45 (2012), no. 37, 374019.

[Hol01] S. Hollands, The Hadamard condition for Dirac fields and adiabatic states on Robertson-Walker spacetimes, Comm. Math. Phys. 216 (2001), no. 3, 635-661.

[Hör71] L. Hörmander, Fourier integral operators. I, Acta Math. 127 (1971), no. 1, 79183.

[Hör83] _ , The Analysis of Linear Partial Differential Operators I. Distribution Theory and Fourier Analysis, Springer, 1983.

[HR02] S. Hollands and W. Ruan, The state space of perturbative quantum field theory in curved spacetimes, Ann. Henri Poincaré 3 (2002), no. 4, 635-657.

[HS12] T.-P. Hack and A. Schenkel, Linear bosonic and fermionic quantum gauge theories on curved spacetimes, Gen. Relat. Gravit. (2012), 1-34.

[Hus94] D. Husemöller, Fibre Bundles, vol. 20, Springer, 1994.

[HW01] S. Hollands and R. M. Wald, Local Wick polynomials and time-ordered products of quantum fields in curved spacetime, Comm. Math. Phys. 223 (2001), no. 2, 289-326.

[HW02] _ Existence of local covariant time ordered products of quantum fields in curved spacetime, Comm. Math. Phys. 231 (2002), 309-345.

[Jon88] P. Jonas, On a class of selfadjoint operators in Krein space and their compact perturbations, Integr. Equat. Operat. Theor. 11 (1988), 351-384, 10.1007/BF01202078.

[Jun95] W. Junker, Adiabatic vacua and Hadamard states for scalar quantum fields on curved spacetime, Ph.D. thesis, University of Hamburg, 1995.

[Kay78] B. S. Kay, Linear spin-zero quantum fields in external gravitational and scalar fields, Comm. Math. Phys. 62 (1978), no. 1, 55-70.

[Kel10] K. J. Keller, Dimensional Regularization in Position Space and a Forest Formula for Regularized Epstein-Glaser Renormalization, Ph.D. thesis, Universität Hamburg, 2010. 
[Lan82] H. Langer, Spectral functions of definitizable operators in Krein spaces, Functional Analysis (D. Butkovic, H. Kraljevic, and S. Kurepa, eds.), Lecture Notes in Mathematics, vol. 948, Springer, 1982, pp. 1-46, 10.1007/BFb0069840.

[LG03] S. Lazzarini and J. M. Gracia-Bondía, Improved Epstein-Glaser renormalization. II. Lorentz invariant framework, J. Math. Phys. 44 (2003), 3863-3875.

[LNT06] H. Langer, B. Najman, and C. Tretter, Spectral theory of the Klein-Gordon equation in Pontryagin spaces, Comm. Math. Phys. 267 (2006), 159-180.

[LNT08] _ Spectral theory of the Klein-Gordon equation in Krein spaces, Proc. Edinburgh Math. Soc. 51 (2008), no. 03, 711-750.

[Man88] C. A. Manogue, The Klein paradox and superradiance, Ann. Phys. 181 (1988), 261-283.

[Mar03] P. Marecki, Quantum Electrodynamics on Background External Fields, Ph.D. thesis, DESY-THESIS-2004-002, 2003.

[Mey98] Y. Meyer, Wavelets, Vibrations, and Scalings, vol. 9, American Mathematical Society, 1998.

[Mor03] V. Moretti, Comments on the stress-energy tensor operator in curved spacetime, Comm. Math. Phys. 232 (2003), 189-221.

[Mor08] _ Q Quantum out-states holographically induced by asymptotic flatness: invariance under space-time symmetries, energy positivity and Hadamard property, Comm. Math. Phys. 279 (2008), 31-75.

[Müh07] R. Mühlhoff, Higher Spin Fields on Curved Spacetimes, Ph.D. thesis, Universität Leipzig, 2007.

[Müh11]__ Cauchy problem and Green's functions for first order differential operators and algebraic quantization, J. Math. Phys. 52 (2011), no. 2, 022303-+.

[Nik07] N. M. Nikolov, Cohomological analysis of the Epstein-Glaser renormalization, arXiv preprint arXiv:0712.2194 (2007).

[NST11] N. Nikolov, R. Stora, and I. Todorov, Configuration space renormalization of massless QFT as an extension problem for associate homogeneous distributions, preprint IHES/P/11/07 (2011).

[Olb07] H. Olbermann, States of low energy on Robertson-Walker space-times, Class. Quantum Grav. 24 (2007), 5011-5030.

[Olv95] P. Olver, Equivalence, Invariants, and Symmetry, Cambridge University Press, 1995.

[Pfä09] F. Pfäffle, Lorentzian manifolds, Quantum Field Theory on Curved Spacetimes, Springer, 2009, pp. 39-58.

[Pra99] D. Prange, Lorentz covariance in Epstein-Glaser renormalization, arXiv preprint hep-th/9904136 (1999).

[Rad96] M. J. Radzikowski, Micro-local approach to the Hadamard condition in quantum field theory on curved space-time, Comm. Math. Phys. 179 (1996), 529-553.

[RS10] M. Ruzhansky and M. Sugimoto, Criteria for Bochner's extension problem, Asymptot. Anal. 66 (2010), no. 3, 125-138.

[San08] K. Sanders, Aspects of Locally Covariant Quantum Field Theory, Ph.D. thesis, University of York, 2008.

[San10] _ Equivalence of the (generalised) Hadamard and microlocal spectrum condition for (generalised) free fields in curved spacetime, Comm. Math. Phys. 295 (2010), 458-501. 
[San12a] _ Essential self-adjointness of Wick squares in quasi-free Hadamard representations on curved spacetimes, J. Math. Phys. 53 (2012), 042502.

[San12b] K. Sanders, Thermal equilibrium states of a linear scalar quantum field in stationary spacetimes, arXiv preprint arXiv:1209.6068 (2012).

[Sch95] G. Scharf, Finite Quantum Electrodynamics: The Causal Approach; 2nd ed., Texts and monographs in physics, Springer, Berlin, 1995.

[SDH12] K. Sanders, C. Dappiaggi, and T.-P. Hack, Electromagnetism, local covariance, the Aharonov-Bohm effect and Gauss' law, arXiv preprint arXiv:1211.6420 (2012).

[Shi91] I. Shigekawa, Spectral properties of Schrödinger operators with magnetic fields for a spin 1/2 particle, J. Funct. Anal. 101 (1991), no. 2, 255 - 285.

[SR50] E. C. G. Stückelberg and D. Rivier, A propos des divergences en théorie des champs quantifiés, Helv. Phys. Acta 23 (1950), 236-239.

[SS70] B. Schroer and J. A. Swieca, Indefinite metric and stationary external interactions of quantized fields, Phys. Rev. 2 (1970), 2938-2943.

[Ste71] O. Steinmann, Perturbation Expansions in Axiomatic Field Theory, Lecture Notes in Physics, vol. 11, Springer, Berlin Springer Verlag, 1971.

[SV00] H. Sahlmann and R. Verch, Passivity and microlocal spectrum condition, Comm. Math. Phys. 214 (2000), 705-731.

[SV01] _ Microlocal spectrum condition and Hadamard form for vector-valued quantum fields In curved spacetime, Rev. Math. Phys. 13 (2001), 1203-1246.

[SVW02] A. Strohmaier, R. Verch, and M. Wollenberg, Microlocal analysis of quantum fields on curved space-times: Analytic wave front sets and Reeh-Schlieder theorems, J. Math. Phys. 43 (2002), 5514.

[Tha92] B. Thaller, The Dirac Equation, Texts and monographs in physics, Springer, Berlin, 1992.

[Ver01] R. Verch, A spin-statistics theorem for quantum fields on curved spacetime manifolds in a generally covariant framework, Comm. Math. Phys. 223 (2001), no. 2, 261-288.

[Ves70] K. Veselić, A spectral theory for the Klein-Gordon equation with an external electrostatic potential, Nucl. Phys. 147 (1970), 215-224.

[Wal77] R. M. Wald, The back reaction effect in particle creation in curved spacetime, Comm. Math. Phys. 54 (1977), 1-19.

[Wal12a] _ The history and present status of quantum field theory in curved spacetime, Einstein and the Changing Worldviews of Physics (C. Lehner, J. Renn, and M. Schemmel, eds.), Einstein Studies, vol. 12, Birkhäuser, 2012, pp. 317-331 (English).

[Wal12b] S. Waldmann, Geometric Wave Equations, arXiv:1208.4706, 2012.

[Wei78] A. Weinstein, The order and symbol of a distribution, Trans. Amer. Math. Soc. 241 (1978), 1-54.

[Wro12] M. Wrochna, Quantum Field Theory in static external potentials and Hadamard states, Ann. Henri Poincaré 13 (2012), 1841-1871.

[Zah13] J. Zahn, The renormalized locally covariant Dirac field, arXiv preprint arXiv:1210.4031 (2013). 